\title{
Time-dependent deformation and associated failure of roof in underground mines
}

\author{
Yuting Xue \\ yuxue@mix.wvu.edu
}

Follow this and additional works at: https://researchrepository.wvu.edu/etd

Part of the Mining Engineering Commons

\section{Recommended Citation}

Xue, Yuting, "Time-dependent deformation and associated failure of roof in underground mines" (2019). Graduate Theses, Dissertations, and Problem Reports. 4077.

https://researchrepository.wvu.edu/etd/4077

This Dissertation is protected by copyright and/or related rights. It has been brought to you by the The Research Repository @ WVU with permission from the rights-holder(s). You are free to use this Dissertation in any way that is permitted by the copyright and related rights legislation that applies to your use. For other uses you must obtain permission from the rights-holder(s) directly, unless additional rights are indicated by a Creative Commons license in the record and/ or on the work itself. This Dissertation has been accepted for inclusion in WVU Graduate Theses, Dissertations, and Problem Reports collection by an authorized administrator of The Research Repository @ WVU.

For more information, please contact researchrepository@mail.wvu.edu. 


\title{
TIME-DEPENDENT DEFORMATION AND ASSOCIATED FAILURE OF ROOF IN UNDERGROUND MINES
}

\author{
Yuting Xue \\ Dissertation submitted \\ to the College of Engineering and Mineral Resources \\ at West Virginia University \\ Doctor of Philosophy in \\ Mining Engineering \\ Brijes Mishra, Ph.D., Chair \\ Keith A. Heasley, Ph.D. \\ Yi Luo, Ph.D. \\ Bruce S. Kang, Ph.D. \\ G.S. Esterhuizen, Ph.D. \\ Department of Mining Engineering
}

in partial fulfillment of the requirements for the degree of

Morgantown, West Virginia

2019

Keywords: Roof fall, coal measure rocks, time-dependent behavior, relaxation test, 3DEC

Copyright 2019 Yuting Xue 


\begin{abstract}
Time-Dependent Deformation and Associated Failure of Roof in Underground Mines
\end{abstract}

\author{
Yuting Xue
}

In underground coal mines, roof falls are a major contributor to injuries and fatalities. Studies have related the occurrence of roof fall to weak immediate roof, high horizontal stress, entry orientation, etc. An often-neglected factor in studies on this topic has been the influence of time-dependent behavior of the roof rock on roof falls. The time-dependent roof failure activity involves both intact and failed rocks. Numerical simulation techniques are available that include time-dependent behavior; however, they lack constitutive models that consider both the intact and the failed behavior of rock. In addition, input properties for the creep models only include the intact rock properties that are determined through constant load (timedependent) tests. For failed rock, standard creep tests cannot be performed on the rock specimens. This thesis aims to understand this behavior through the following steps:

- Develop a new laboratory test method

- Develop a new constitutive model that incorporates the pre- and post-failure behavior

- Implement the constitutive model into 3DEC

- Analyze the hypothetical mine geometry using the new constitutive model

First, this study develops a new relaxation equation based on Burgers Model. Relaxation tests studied rock specimens in both intact and failed stages. The results from the tests showed significant difference in the viscous property between intact and failed rocks. The results of these relaxation tests determined the viscous parameters with the new relaxation equation. Next, this study constructed numerical models of laboratory sized specimens in 3DEC software. The models incorporated the new relaxation equation, and model runs showed that stress relaxation is significantly present in the post-failure region, rather than in the pre-failure region. Further, a single entry mine model in 3DEC analyzed the influence of strength degradation and the variation in the viscous property on the time-dependent failure process. Variation in the viscous parameter showed significant effects on the failure process in the rock mass.

A series of unconfined relaxation tests was performed on sandstone specimens and coal measure rocks. For sandstone, specimens were cored from sandstone blocks; for coal measure rocks, which include shale, sandy shale, and shaly limestone, cores were obtained from mine sites. The test results show that the relaxation behaviors of intact and failed rock specimens are different. The stress relaxation curves in the pre-failure region showed a typical, smooth stress relaxation behavior, while the stress relaxation demonstrates stepped behavior in the post-failure region. For coal measure rocks, the variation in the timedependent properties of failed rock were insignificant.

A viscoelastic-strain-softening constitutive model was developed by incorporating both time-dependent and strain-softening behavior. The model was included in the 3DEC software as a user-defined model. Parametric model runs based on the time-dependent laboratory tests verified the accuracy of the proposed model. Finally, the user-defined model, using hypothetical condition, investigated the influence of various factors on the time-dependent deformation and failure of massive and bedded roof. These simulations investigated the influence of directional horizontal stress, step-wise excavation, and bedding planes on the time-dependent response of mine roof. This research achieved a comprehensive understanding of the timedependent formation of roof fall. 


\section{ACKNOWLEDGEMENT}

I would like to express my sincere gratitude to my advisor Dr. Brijes Mishra for his continuous support of my doctoral research effort and also for his patience and motivation.

I would also like to thank my graduate committee members Drs. Keith Heasley, Yi Luo, Bruce Kang, and G.S. Esterhuizen, for their insightful comments and suggestions. I appreciate the valuable time they have spent on my graduate committee.

My sincere thanks to Dr. Syd Peng. His passion for ground control and his concern for the future research also encouraged me to pursue my research. The Syd S. and Felicia F. Peng ground control scholarship motivated me further to continue this research. In addition, the ICGCM conference gave me the chance to meet worldwide researchers in this area.

In addition, I would like to thank the colleagues including Danqing Gao, $\mathrm{Xu}$ Tang, Neel Gupta, Bonaventura A. Mangu Bali, Debashis Das and Prasoon Garg and my Chinese friends Hua Jiang, Jian Yang, Yan Ye, Kaifang Li, Ming Fan, Mingming Li, Wan Wang, Meng Zhang. I also would like to thank visiting scholars from China, Drs. Feng Du, Shiming Wei and Guorui Feng for their inputs on my research.

I am grateful to thank Karen, Genette and Karla for providing the administrative support throughout my stay at WVU.

Finally, I would like to thank my parents and my wife, Mingming Shi, for supporting me spiritually throughout writing this dissertation and my life in general.

Thanks for all your encouragement! 


\section{Table of Contents}

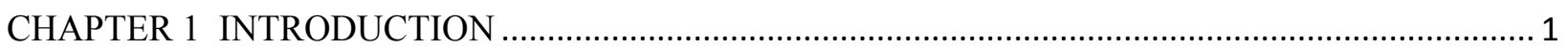

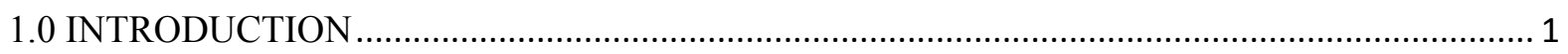

1.2 RESEARCH QUESTON AND OBJECTIVE ................................................................... 2

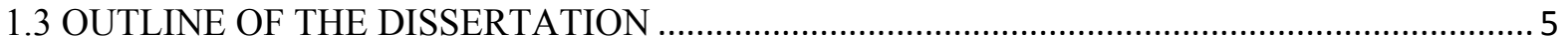

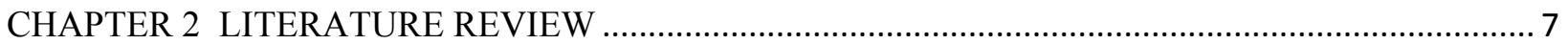

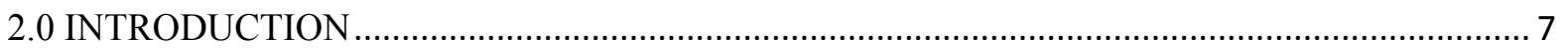

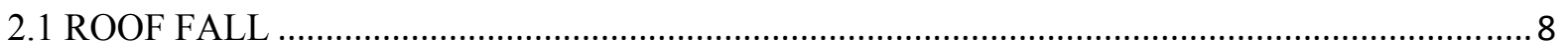

2.2 TIME-DEPENDNT BEHAVIOR OF INTACT ROCK …........................................................ 15

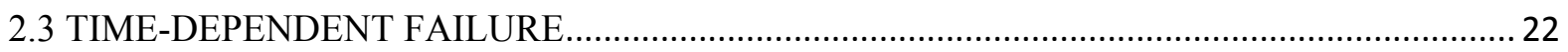

2.3.1 Laboratory study on the time-dependent failure of rock ..................................................... 22

2.3.2 Incorporation of time-dependent failure into constitutive model............................................ 23

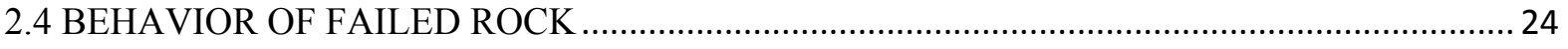

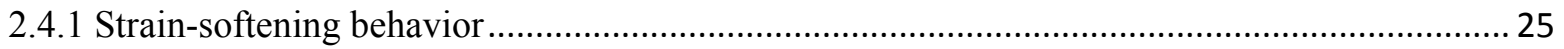

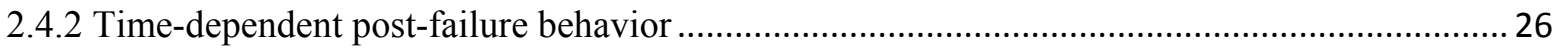

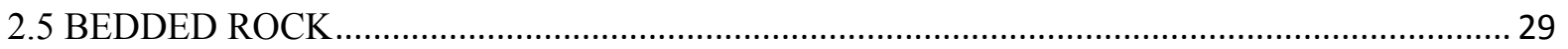

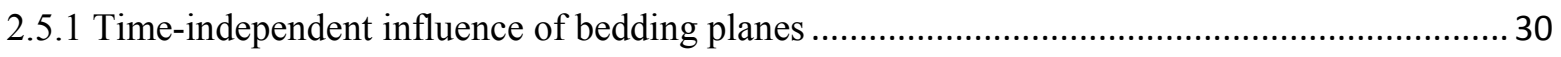

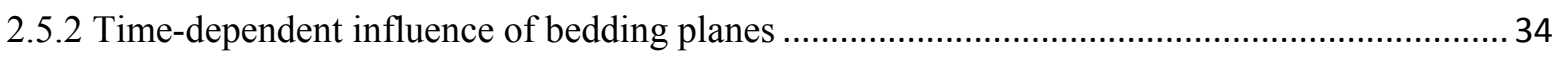

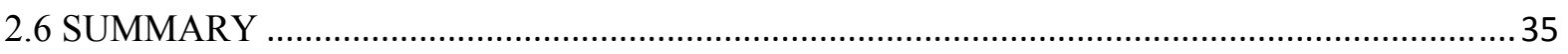

CHAPTER 3 STUDY OF THE TIME-DEPENDENT PROPERTY OF ROCK WITH RELAXATION

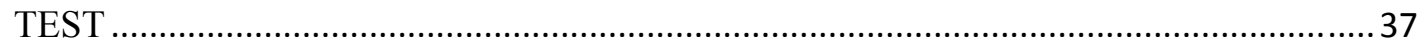

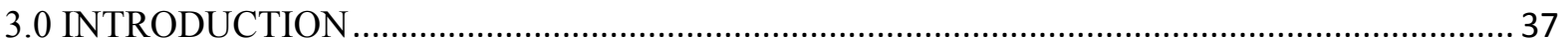

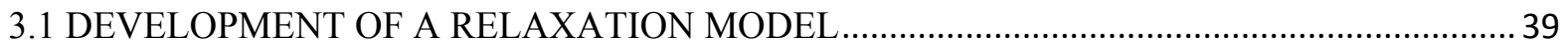

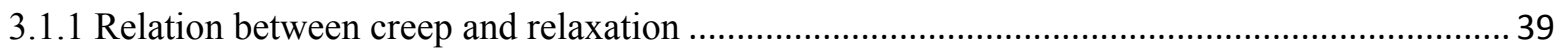

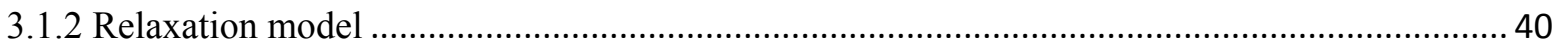

3.2 VERIFICATION OF USING RELAXATION TEST TO DETERMINE THE TIME-

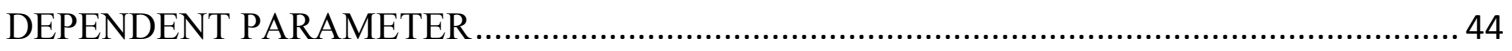

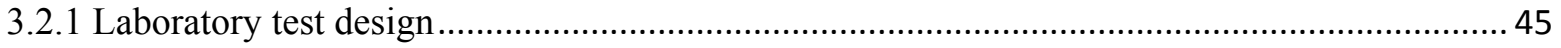

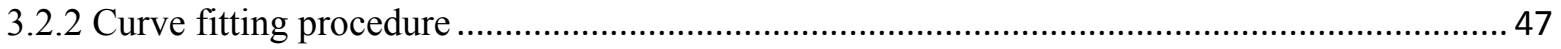

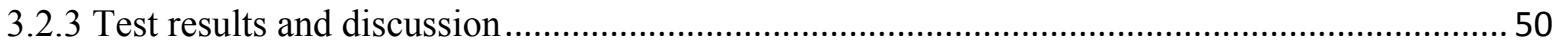

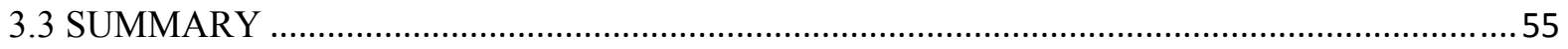

CHAPTER 4 THE INFLUENCE OF THE VARIATION IN THE TIME-DEPENDENT PROPERTY AND STRENGTH DEGRADATION ON TIME-DEPENDENT ROOF FAILURE ..........56 
4.1 STUDY ON THE VARIATION IN THE TIME-DEPENDENT PROPERTY WITH RELAXATION TEST AND ITS INFLUENCE ON TIME-DEPENDENT ROOF FAILURE....57

4.1.1 Variation in the time-dependent property of rock ................................................................ 58

4.1.2 Simulation methodology to study the influence of variation in the time-dependent property...61

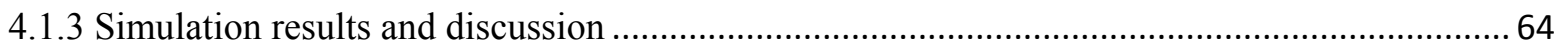

4.2 INFLUENCE OF STRENGTH DEGRADATION ON TIME-DEPENDENT ROOF FAILURE 70

4.2.1 Effective viscous strain for Burgers materials .................................................................... 71

4.2.2 Incorporation of strength degradation into numerical simulation .......................................... 73

4.2.3 Numerical study on the influence of strength degradation on roof behavior ............................ 74

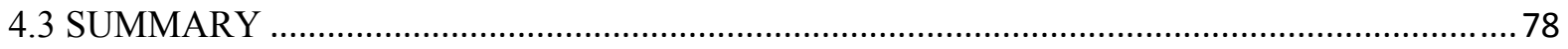

CHAPTER 5 LABORATORY STUDY ON THE TIME-DEPENDENT BEHAVIOR OF INTACT AND FAILED ROCKS WITH UNCONFINED RELAXATION TEST ...................................... 80

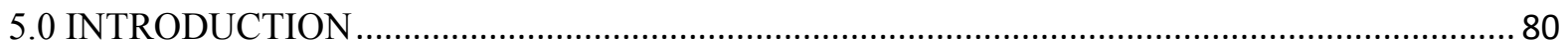

5.1 THE TIME-DEPENDENT BEHAVIOR OF INTACT AND FAILED SANDSTONE

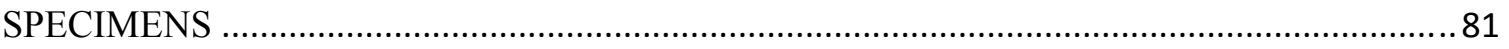

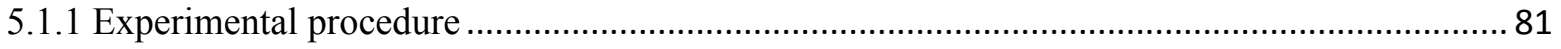

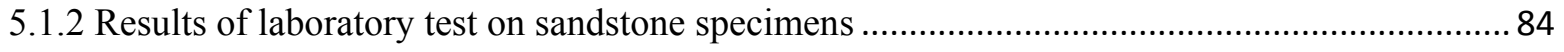

5.1.3 Variation in the time-dependent property of coal measure rocks ............................................ 90

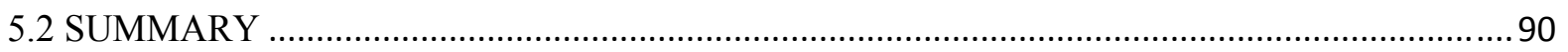

CHAPTER 6 DEVELOPMENT OF A VISCOELASTIC-STRAIN-SOFTENING MODEL ...................92

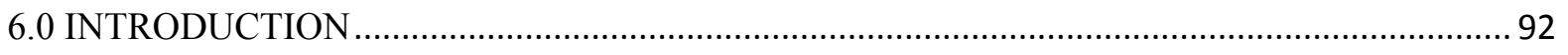

6.1 DEVELOPMENT OF A NEW VISCOELASTIC-STRAIN-SOFTENING MODEL ...................93

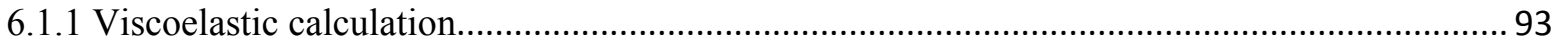

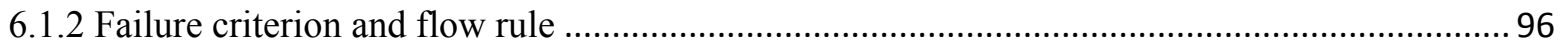

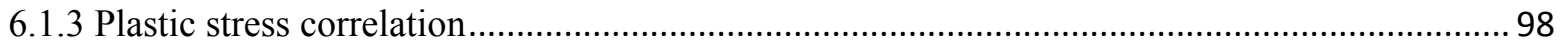

6.1.4 Variation in the time-dependent property ........................................................................... 99

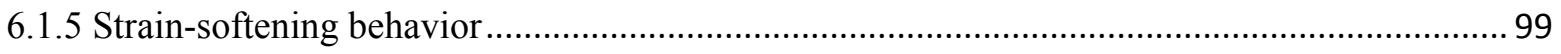

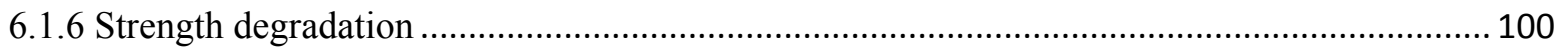

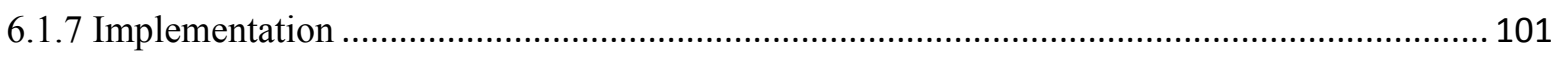

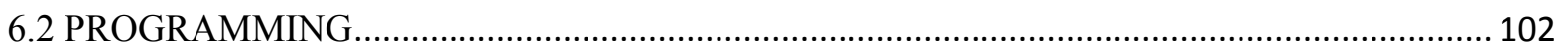

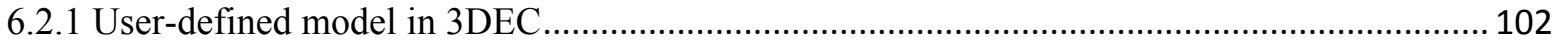

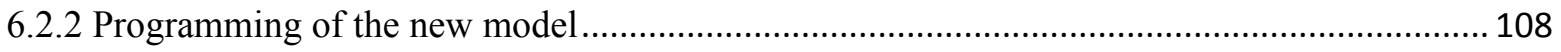

6.3 MODEL PARAMETER DETERMINATION AND CALIBRATION …................................... 109 


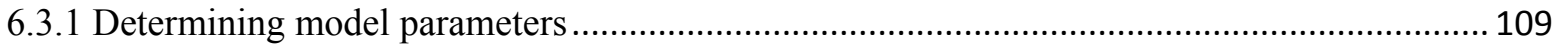

6.3.2 Calibration for strain-softening behavior ......................................................................... 110

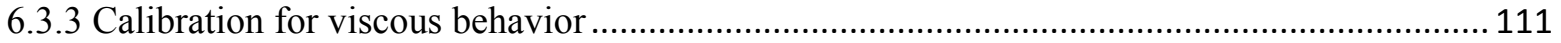

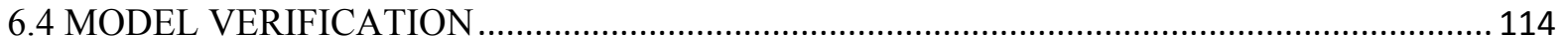

6.4.1 Numerical simulation of unconfined compression test ..................................................... 115

6.4.2 Unconfined compression test with relaxation tests .............................................................. 115

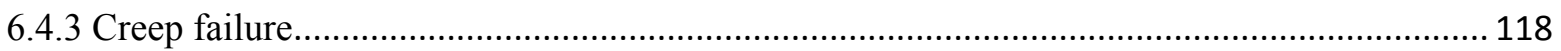

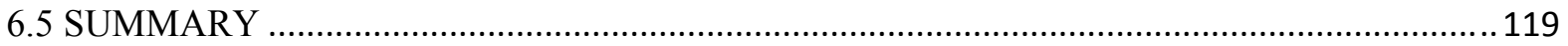

CHAPTER 7 NUMERICAL STUDY ON THE TIME-DEPENDENT BEHAVIOR OF MASSIVE

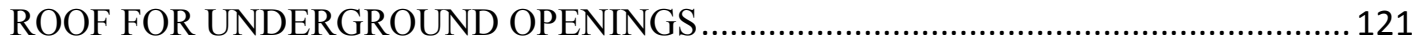

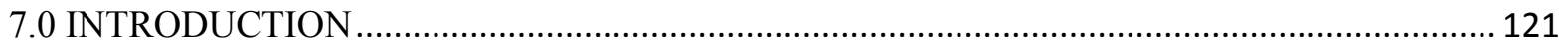

7.1 STUDY ON THE TIME-DEPENDENT BEHAVIOR OF MASSIVE MINE ROOF .................122

7.2 INFLUNECE OF STRESS RATIO ON THE TIME-DEPENDENT FAILURE OF MINE ROOF

7.3 INFLUENCE OF STEP-WISE EXCAVATION ON THE TIME-DEPENDENT FAILURE OF

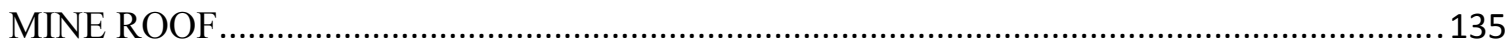

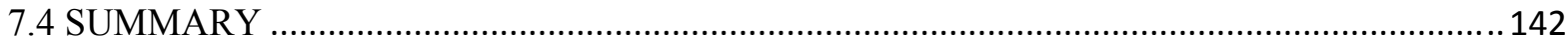

CHAPTER 8 NUMERICAL STUDY ON THE TIME-DEPENDENT BEHAVIOR OF BEDDED ROOF

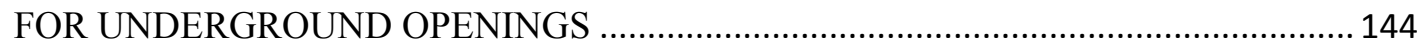

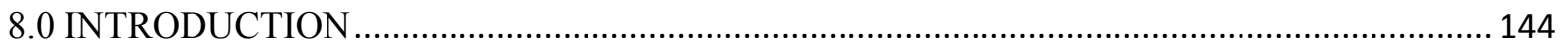

8.1 TWO-DIMENSIONAL BEAM ANALYSIS OF BEDDED ROOF .......................................... 145

8.1.1 Time-independent and time-dependent behaviors of single intact beam ................................ 146

8.1.2 Time-independent and time-dependent behaviors of bedded beam ....................................... 151

8.2 TIME-DEPENDENT SIMULATION OF BEDDED MINE ROOF ......................................... 156

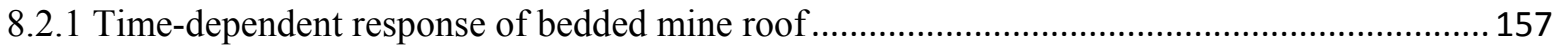

8.2.2 Influence of bedding plane strength and stiffness on the time-dependent behavior of bedded

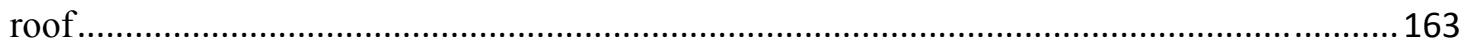

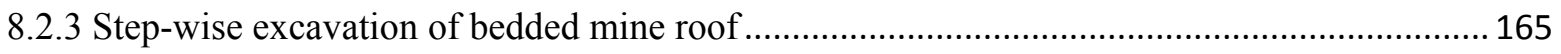

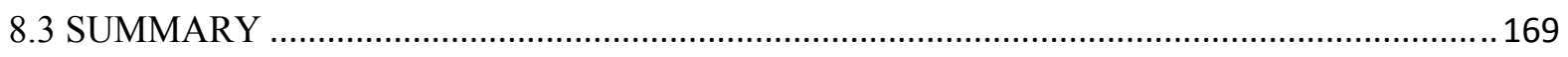

CHAPTER 9 CONCLUSIONS AND RECOMMENDED FUTURE STUDIES ..................................171

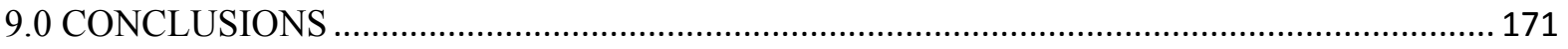

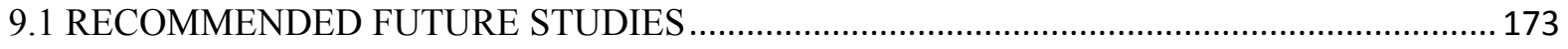

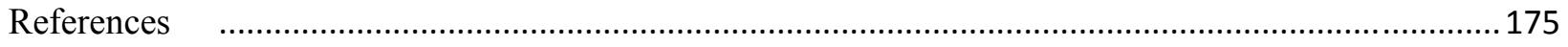




\section{List of Figures}

\section{Chapter 1}

Fig. 1.1. Historical roof and rib fall fatality rates. 1

Fig. 1.2. Comparison between roof convergence (line), micro-seismic event frequency (dot) and local

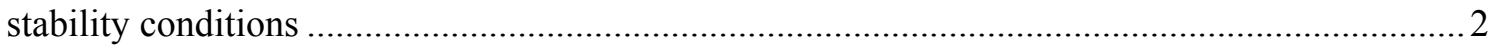

Fig. 1.3. Cutter failure process in underground coal mines .............................................................. 3

\section{Chapter 2}

Fig. 2.1. Distribution of cohesion showing cutter development ............................................................. 10

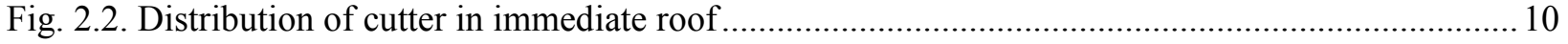

Fig. 2.3. Plan view showing effect of a change in the orientation of the maximum horizontal stress potential roof failure in a room and pillar layout consisting of square pillars

Fig. 2.4. Plan view showing effects of a change in the pillar offset on potential failure in a room and pillar layout consisting of rectangular pillars.

Fig. 2.5. Numerical simulation of bedded roof failure with different interface properties 12

Fig. 2.6. Maximum principal stress distribution on a plane $0.25 \mathrm{~m}$ into immediate roof of a tunnel 13

Fig. 2.7. Development of shear cracks in the roadway roof during stress release 14

Fig. 2.8. Development of tension crack in the roadway roof during stress release 14

Fig. 2.9. Damage development in the roadway roof during stress release. 15

Fig. 2.10. Typical strain-time curve under creep condition ... 16

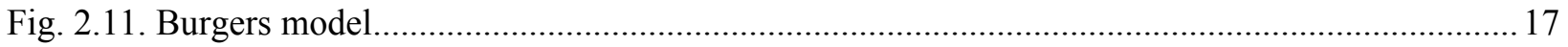

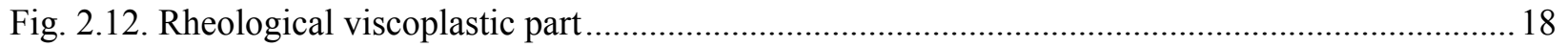

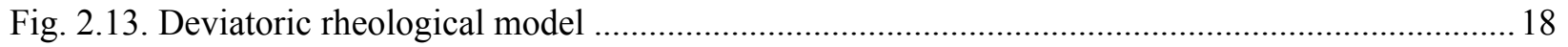

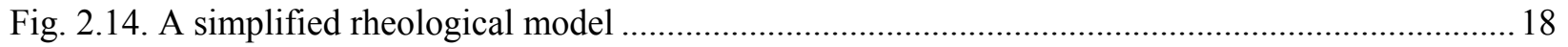

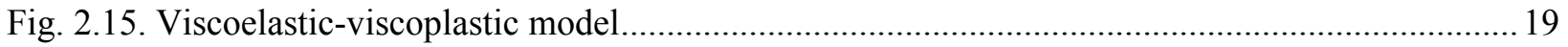

Fig. 2.16. The evolution of yield surface for intact rock to the eventual residual surface for failed rock .. 20

Fig. 2.17. Limit surfaces and stress fields in the principal stress plane for the stress hardening elasto-

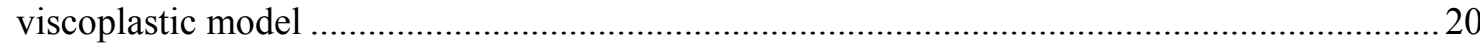

Fig. 2.18. Ground reaction curves for materials with different stress-strain relationships ....................... 25

Fig. 2.19. Creep curves of fractured Arkose sandstone ......................................................................... 26

Fig. 2.20. Stress-strain curves with different strain rates ................................................................... 27

Fig. 2.21. Hypothetical variation in complete force-displacement curves due to relaxation alone ............28

Fig. 2.22. Relaxation behavior for the three tests initiated in post-failure region.................................... 29

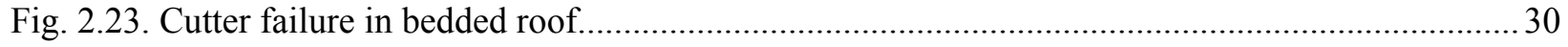

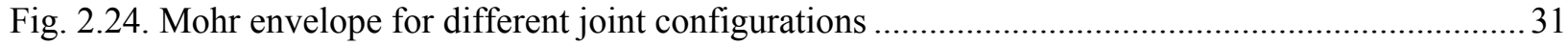

Fig. 2.25. Breakage patterns of centrifuged models with layer thickness less than half the roof span.......32 
Fig. 2.26. Breakage pattern of centrifuged models with a layer thickness greater than half the roof span, $\mathrm{H} / \mathrm{l}=0.57$

Fig. 2.27. Normalized horizontally laminated anisotropic ground behavior chart showing when anisotropic plasticity analysis should be conducted and generally what type of anisotropic behavior can be expected.

\section{Chapter 3}

Fig. 3.1. Development of fracture zone 24 hours after the 9 th mining increment. The stope is represented by the thick black line 37

Fig. 3.2. Relaxation test results by Hudson and Brown ......................................................................... 38

Fig. 3.3. Schematic for numerical simulation of unconfined relaxation test ........................................... 40

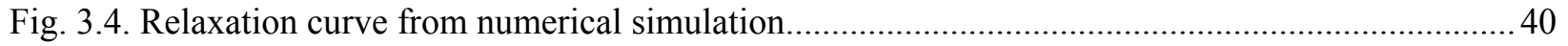

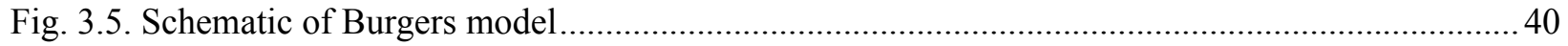

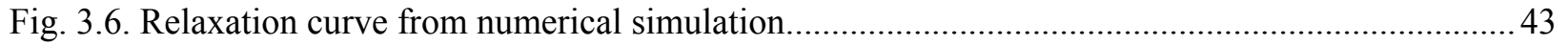

Fig. 3.7. Theoretically calculated creep and relaxation curves with same parameters ............................ 44

Fig. 3.8. MTS servo-controlled compression testing machine and its components.................................. 45

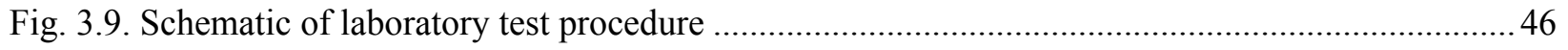

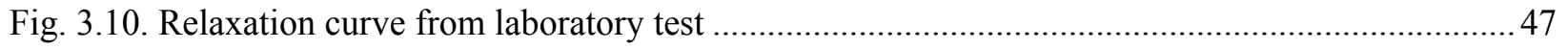

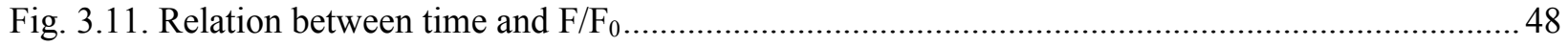

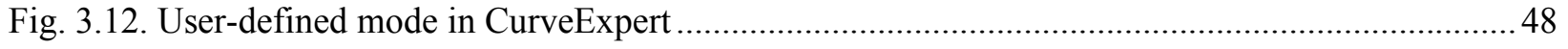

Fig. 3.13. Screen to input guess values for the three variables .............................................................. 49

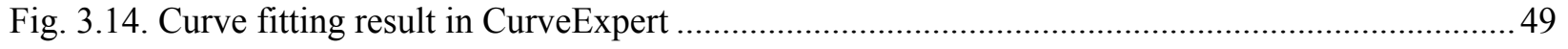

Fig. 3.15. Curve fitting result of Burgers model for creep test ............................................................ 50

Fig. 3.16. Curve fitting result of Burgers model for relaxation test ........................................................5 50

Fig. 3.17. Values of $\eta \mathrm{m}\left(\mathrm{GPa}^{*} \mathrm{~s}\right)$ for different rocks with creep and relaxation tests from second group of

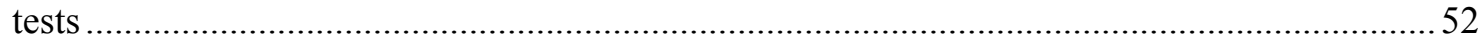

Fig. 3.18. Values of Ek (GPa) for different rocks with creep and relaxation tests from second group of tests.

Fig. 3.19. Values of $\eta \mathrm{k}\left(\mathrm{GPa}^{*} \mathrm{~s}\right)$ for different rocks with creep and relaxation tests from second group of tests.....

Fig. 3.20. Relaxation tests in residual stage .53

Fig. 3.21. Failure during a relaxation test

\section{Chapter 4}

Fig. 4.1. Time to failure at different constant stress levels during the creep tests on Westerly Granite......57

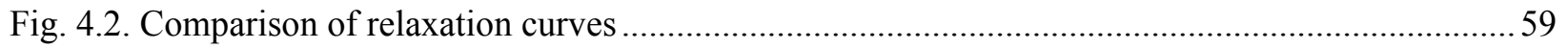

Fig. 4.3. Comparison of relaxation curves, starting from same stress values, before and after failure ...... 60

Fig. 4.4. Flowchart showing communication between 3DEC and FISH function................................... 61 
Fig. 4.5. Model for unconfined compression test with relaxation tests

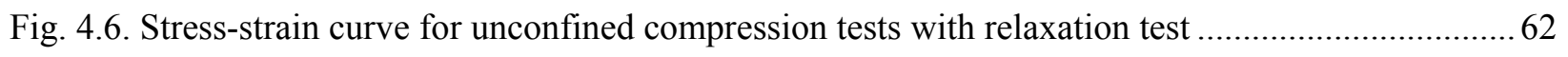

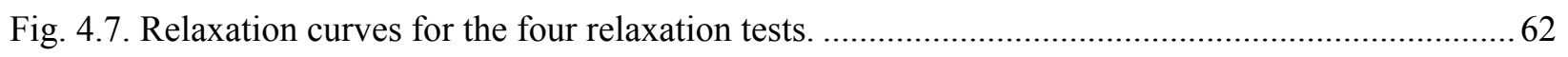

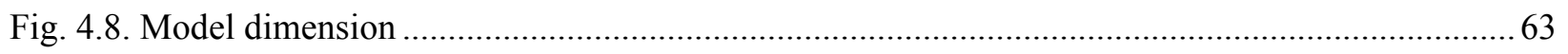

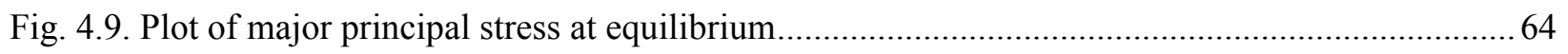

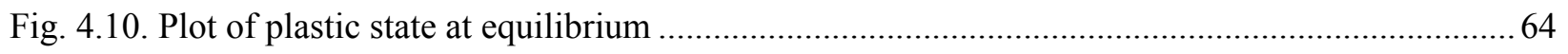

Fig. 4.11. Plot of major principal stress after one day for model with constant parameters ...................... 65

Fig. 4.12. Plot of plastic state after one day for model with constant parameters ..................................... 65

Fig. 4.13. Illustration of "synchronized" stress relaxation in plastic and elastic zone for the model with

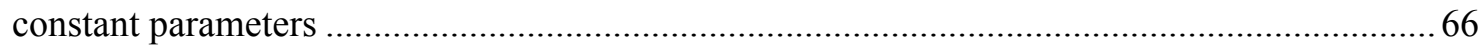

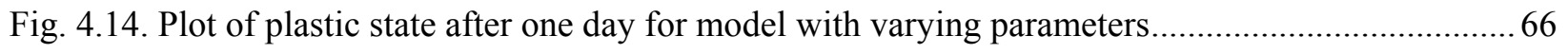

Fig. 4.15. Plot of major principal stress after one day for model with varying parameters ........................ 67

Fig. 4.16. Illustration of stress relaxation in plastic and elastic zone for the model with varying parameters 67

Fig. 4.17. Illustration of mechanism for time-dependent propagation of failure ..................................... 68

Fig. 4.18. Comparison of roof convergence for the models with constant and varying parameters ........... 69

Fig. 4.19. Stress-strain curve for the simulated unconfined creep test with Burgers model and Mohr-

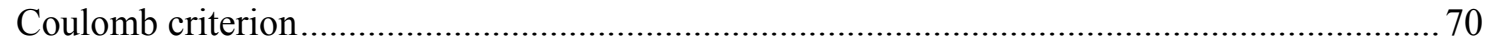

Fig. 4.20. Schematic of viscous part in Burgers model for one dimension.............................................. 72

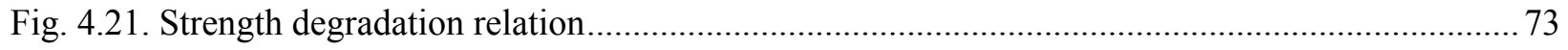

Fig. 4.22. Relation between friction and effective viscous strain used in the simulation ........................ 73

Fig. 4.23. Creep curve showing three stages for the specimen considering strength degradation.............. 74

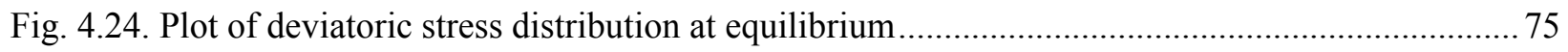

Fig. 4.25. Plot of maximum shear stress distribution at equilibrium ..................................................... 75

Fig. 4.26. Plot of plastic state after one day for model with varying parameters and strength degradation 76

Fig. 4.27. Plot of effective viscous strain after one day for the model with constant parameters and

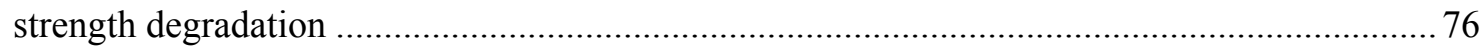

Fig. 4.28. Plot of friction after one day for the model with constant parameters and strength degradation

Fig. 4.29. Plot of plastic state after two minutes for the model with varying parameters and strength degradation .77

Fig. 4.30. Plot of plastic state after one day for model with varying parameters and strength degradation 77

\section{Chapter 5}

Fig. 5.1. Photos of specimen before and after unconfined compression test ......................................... 81

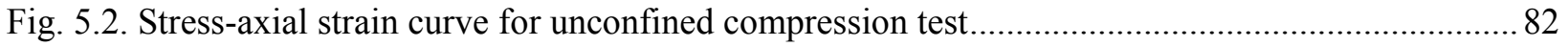

Fig. 5.3. Stress-lateral deformation curve for unconfined compression tests ........................................ 82 


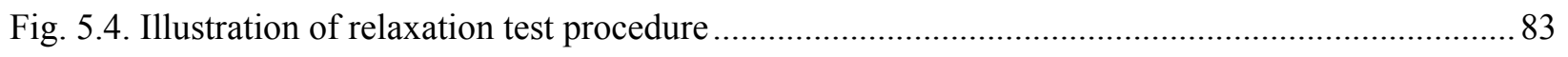

Fig. 5.5. Specimen pictures for test No.7 with non-persistent major failure plane................................... 84

Fig. 5.6. Specimen pictures for test No.9 with persistent major failure plane ......................................... 84

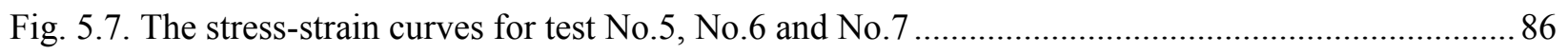

Fig. 5.8. Relation between stress ratio starting relaxation and relaxed stress ratio in the pre-failure region

Fig. 5.9. The relation between time and stress ratio during the relaxation tests in the pre-failure and post-

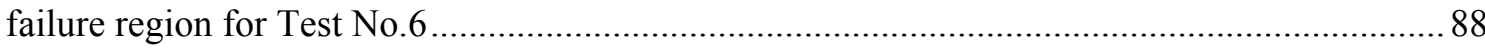

Fig. 5.10. Relaxation curves resulted from different viscous parameters ................................................ 89

\section{Chapter 6}

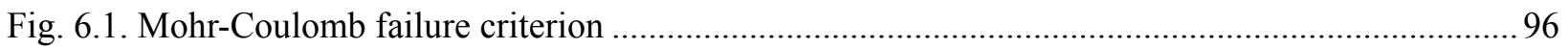

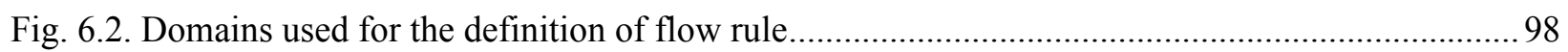

Fig. 6.3. Approximation of cohesion variation with plastic train by segments..................................... 100

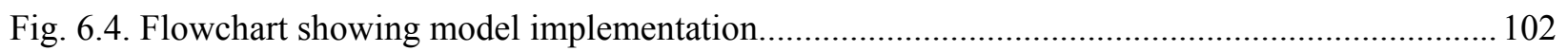

Fig. 6.5. Model to consider strain softening behavior with different failure band thicknesses................ 110

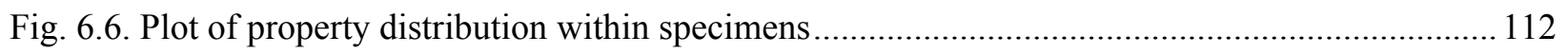

Fig. 6.7. Stress-strain curves for the specimens with bands of different thicknesses ............................ 113

Fig. 6.8. Stress-time curves for the specimens with bands of different thicknesses .............................. 113

Fig. 6.9. Stress-strain curves for the specimens with bands of different thicknesses after correction ...... 114

Fig. 6.10. Stress-time curves for the specimens with bands of different thicknesses after correction...... 114

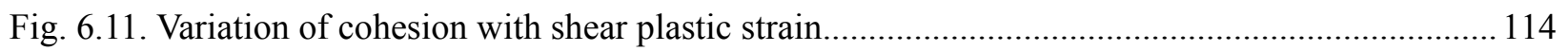

Fig. 6.12. Variation of friction angle with effective viscous strain ..................................................... 115

Fig. 6.13. Comparison of strain-softening behaviors between the strain-softening model and vess model 115

Fig. 6.14. Stress-strain curves with cvis and vess model with perfect plasticity .................................. 116

Fig. 6.15. Relaxation curves with cvis and vess model with perfect plasticity.................................... 117

Fig. 6.16. Stress-strain curve with vess model considering strain-softening behavior and variation in the time-dependent property

Fig. 6.17. Relaxation curves with vess model considering strain-softening behavior and variation in the time-dependent property.....

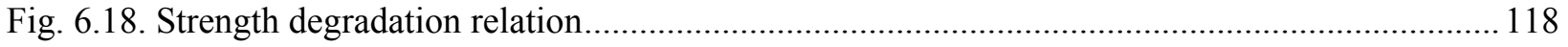

Fig. 6.19. Results of simulated creep tests with strength degradation .................................................. 119

Fig. 6.20. Time to failure with different constant stresses .................................................................. 119

Chapter 7

Fig. 7.1. Mine model with massive roof 122

Fig. 7.2. Distribution of major principal stress at equilibrium 123 


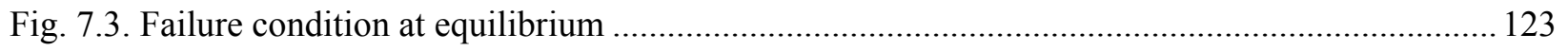

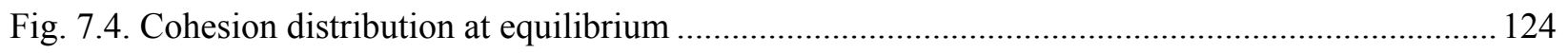

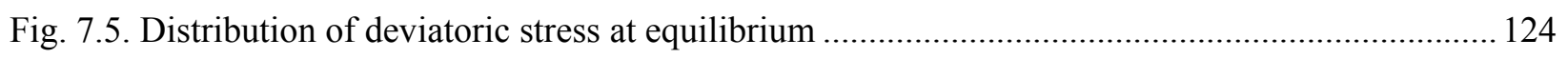

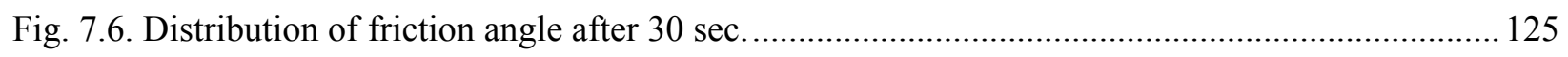

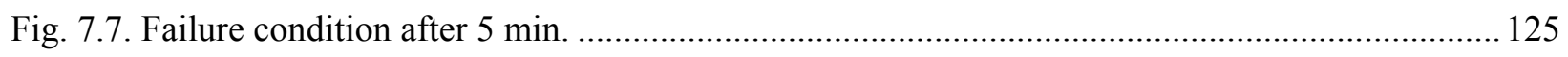

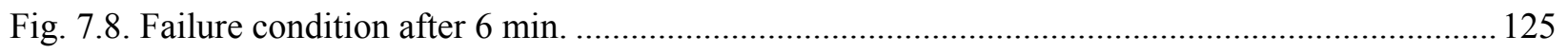

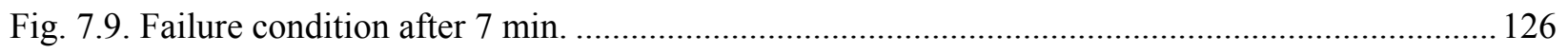

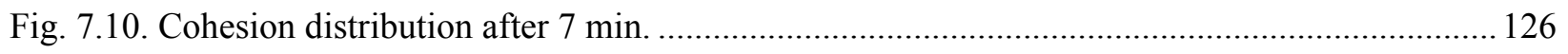

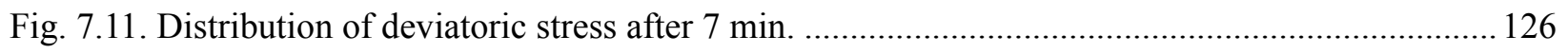

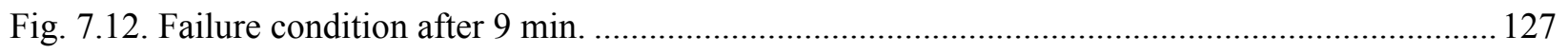

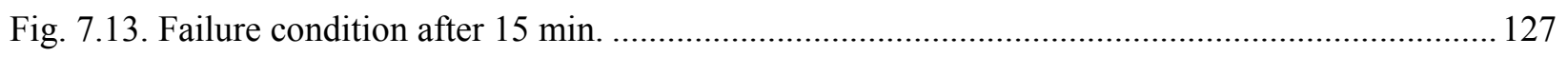

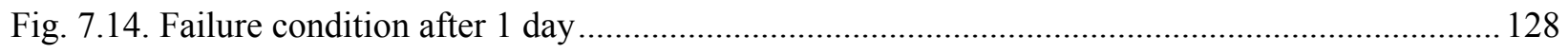

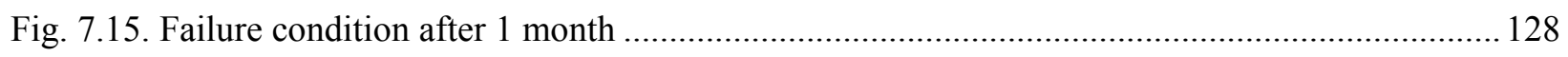

Fig. 7.16. Distribution of major principal stress after 1 month .......................................................... 128

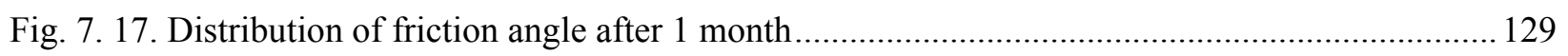

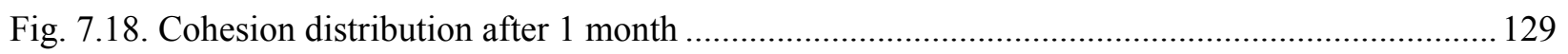

Fig. 7.19. Plot of time-dependent displacement of monitoring points after 1 day .................................. 130

Fig. 7.20. Plot of time-dependent deformation of the monitoring points after 1 month ......................... 130

Fig. 7.21. Plot of maximum unbalance force during creep simulation of 1 day ..................................... 131

Fig. 7.22. Plot of maximum unbalance force during creep simulation of $15 \mathrm{~min}$.................................. 131

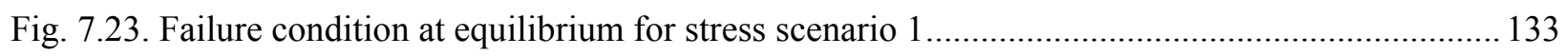

Fig. 7.24. Distribution of maximum shear stress at equilibrium for stress scenario 1 .......................... 133

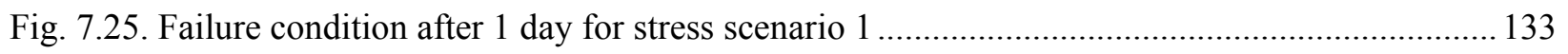

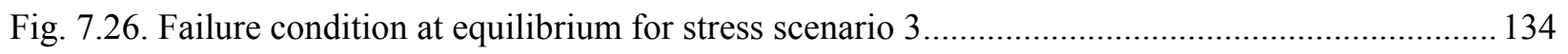

Fig. 7.27. Distribution of maximum shear stress at equilibrium for stress scenario 3 .......................... 134

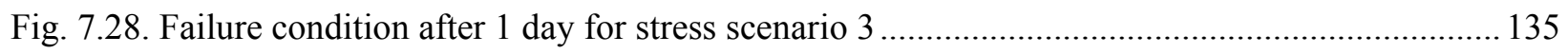

Fig. 7.29. Failure condition at the monitoring location after the $26 \mathrm{th}(26 \mathrm{ft})$ mining step...................... 137

Fig. 7.30. Distribution of maximum shear stress along the right edge of the entry after the 26th (26 ft)

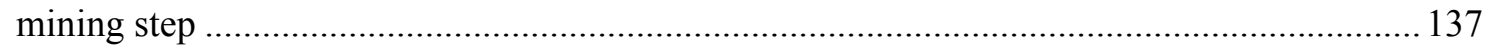

Fig. 7.31. Distribution of friction angle at the monitoring location after the 26th (26 ft) mining step ..... 137

Fig. 7.32. Distribution of friction angle at the monitoring location after the $26 \mathrm{th}(26 \mathrm{ft})$ mining step ..... 138

Fig. 7.33. Failure condition at the monitoring location after the 30th $(30 \mathrm{ft})$ mining step..................... 138

Fig. 7.34. Cohesion distribution at the monitoring location after the 30th (30 ft) mining step .............. 138

Fig. 7.35. Distribution of friction angle at the monitoring location after the 30th (30 ft) mining step ..... 139

Fig. 7.36. Displacement distribution at the monitoring location after the 30 th (30 ft) mining step.......... 139 
Fig. 7.37. Failure condition at the monitoring location after the $35 \mathrm{th}(35 \mathrm{ft})$ mining step...................... 140

Fig. 7.38. Cohesion distribution at the monitoring location after the 35th (35 ft) mining step ............... 140

Fig. 7.39. Distribution of friction angle at the monitoring location after the 35 th ( $35 \mathrm{ft})$ mining step ..... 140

Fig. 7.40. Displacement distribution at the monitoring location after the 35 th ( $35 \mathrm{ft}$ ) mining step......... 140

Fig. 7.41. Failure condition at the monitoring location after the 50th $(50 \mathrm{ft})$ mining step..................... 141

Fig. 7.42. Displacement distribution at the monitoring location after the 50th (50 ft) mining step......... 141

Fig. 7.43. The relation between displacement and time for the various monitoring points within the mine roof . 142

Fig. 7.44. The time-dependent vertical displacement of one monitoring point after six mining steps ..... 142

\section{Chapter 8}

Fig. 8.1. Cutter failure in bedded roof.

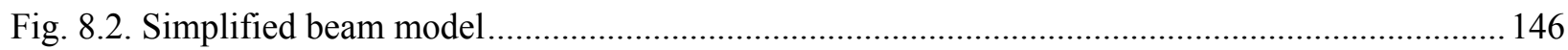

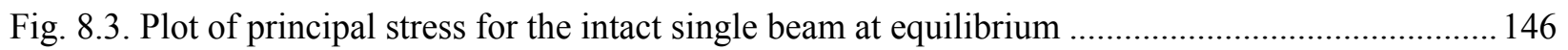

Fig. 8.4. Plot of major principal stress for the intact single beam at equilibrium

Fig. 8.5. Plot of major principal stress for the intact single beam at equilibrium when only vertical boundary stress is applied

Fig. 8.6. Plot of major principal stress for the intact single beam at equilibrium when vertical boundary stress and 1.9 MPa of initial horizontal stress is applied.

Fig. 8.7. Plot of major principal stress for the intact single beam at equilibrium when vertical boundary stress and 3.6 MPa of initial horizontal stress is applied.....

Fig. 8.8. Plot of major principal stress for the intact single beam at equilibrium when vertical boundary stress and 5.4 MPa of initial horizontal stress is applied.. 148

Fig. 8.9. Plot of major principal stress for single intact beam at equilibrium.... 149

Fig. 8.10. Plot of major principal stress for single intact beam after one day. 150

Fig. 8.11. Plot of major principal stress for single intact beam after one week 150

Fig. 8.12. Plot of principal stress for single intact beam after one week 151

Fig. 8.13. Plot of principal stress for $0.8 \mathrm{~m}$-thick beam at equilibrium 152

Fig. 8.14. Plot of principal stress for $0.4 \mathrm{~m}$-thick beam at equilibrium 152

Fig. 8.15. Plot of principal stress for $0.2 \mathrm{~m}$-thick beam at equilibrium 152

Fig. 8.16. Plot of major principal stress for $0.4 \mathrm{~m}$-thick beam at equilibrium .... 153

Fig. 8.17. Plot of major principal stress for $0.2 \mathrm{~m}$-thick beam at equilibrium .... 153

Fig. 8. 18. Plot of major principal stress for $0.4 \mathrm{~m}$-thick beam at equilibrium 154

Fig. 8.19. Plot of major principal stress for $0.2 \mathrm{~m}$-thick beam at equilibrium .... 154

Fig. 8.20. Stress analysis between one bedding plane without strength 154

Fig. 8.21. Plot of major principal stress for $0.8 \mathrm{~m}$-thick beam with low bedding plane strength at equilibrium 155 
Fig. 8.22. Plot of major principal stress for $0.8 \mathrm{~m}$-thick beam with low bedding plane strength at equilibrium.

Fig. 8.23. Plot of major principal stress for $0.8 \mathrm{~m}$-thick beam with low bedding plane strength at equilibrium.

Fig. 8.24. Plot of shear stress within bedding plane with different strengths

Fig. 8.25. Mine model with bedded roof.

Fig. 8.26. Plot of major principal stress distribution at equilibrium for the model with massive roof .... 158

Fig. 8.27. Plot of maximum shear stress distribution at equilibrium for the model with massive roof ... 158

Fig. 8.28. Plot of failure condition and joint normal stress magnitude at equilibrium for the model with bedded roof.

Fig. 8.29. Plot of major principal stress and joint normal stress magnitude at equilibrium for the model with bedded roof.

Fig. 8.30. Plot of failure condition and joint normal stress magnitude at equilibrium for the model with bedded roof.

Fig. 8.31. Plot of maximum shear stress and joint shear stress magnitude at equilibrium for the model with bedded roof

Fig. 8.32. Plot of deviatoric stress at equilibrium for the model with bedded roof .

Fig. 8.33. Plot of failure condition after one day for the model with bedded roof

Fig. 8.34. Plot of major principal stress and joint normal stress after one day for the model with bedded roof

Fig. 8.35. Plot of maximum shear stress and joint shear stress after one day for the model with bedded roof

Fig. 8.36. Plot of displacement magnitude and joint normal displacement magnitude after one hour for the model with bedded roof.

Fig. 8.37. Plot of failure condition at equilibrium for the model with bedding planes of low strength and stiffness

Fig. 8.38. Plot of displacement and joint shear stress magnitude at equilibrium for the model with bedding planes of low strength and stiffness....

Fig. 8.39. Plot of failure condition after one day for the model with bedding planes of low strength and stiffness

Fig. 8.40. Plot of displacement and joint shear stress magnitude after one day for the model with bedding planes of low strength and stiffness

Fig. 8.41. Plot of displacement and joint shear stress magnitude after one week for the model with bedding planes of low strength and stiffness .....

Fig. 8.42. Plot of failure condition and joint shear stress at 26th mining step 166

Fig. 8.43. Plot of failure condition and joint shear stress at 30th mining step 166

Fig. 8.44. Plot of failure condition and joint shear stress at 35th mining step 167

Fig. 8.45. Plot of failure condition and joint shear stress at 50th mining step 167 
Fig. 8.46. Plot of displacement magnitude and joint normal displacement magnitude at 26th mining step 168

Fig. 8.47. Plot of displacement magnitude and joint normal displacement magnitude at 50th mining step 168 


\section{List of Tables}

\section{Chapter 3}

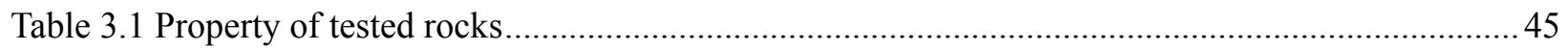

Table 3.2 Comparison of creep parameters from creep and relaxation tests from first group of test ......... 51

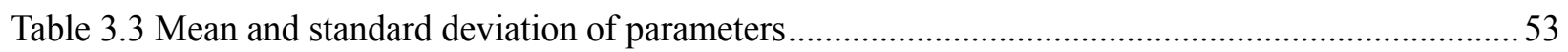

\section{Chapter 4}

Table 4.1 Parameters in the post-failure region determined from Hudson and Brown paper ....................59

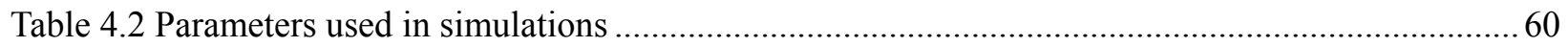

\section{Chapter 5}

Table 5.1 Unconfined compression test result summary ................................................................... 82

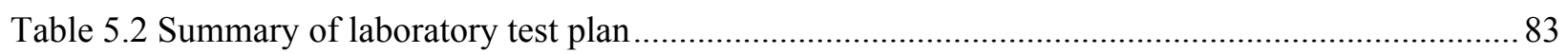

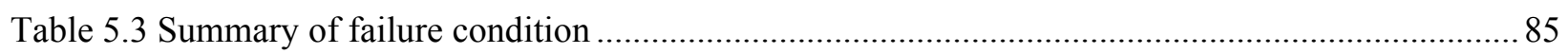

Table 5.4 Summary of the averaged viscous parameters for intact and failed rock ................................. 88

Table 5. 5 Summary of the averaged viscous parameters based on the failure condition.......................... 89

Table 5.6 Summary of parameters determined from the laboratory tests ............................................... 90

\section{Chapter 6}

Table 6.1 Parameters for model calibration and verification

\section{Chapter 7}

Table 7.1 In-situ stress scenarios used in the simulations 


\section{CHAPTER 1 \\ INTRODUCTION}

\subsection{INTRODUCTION}

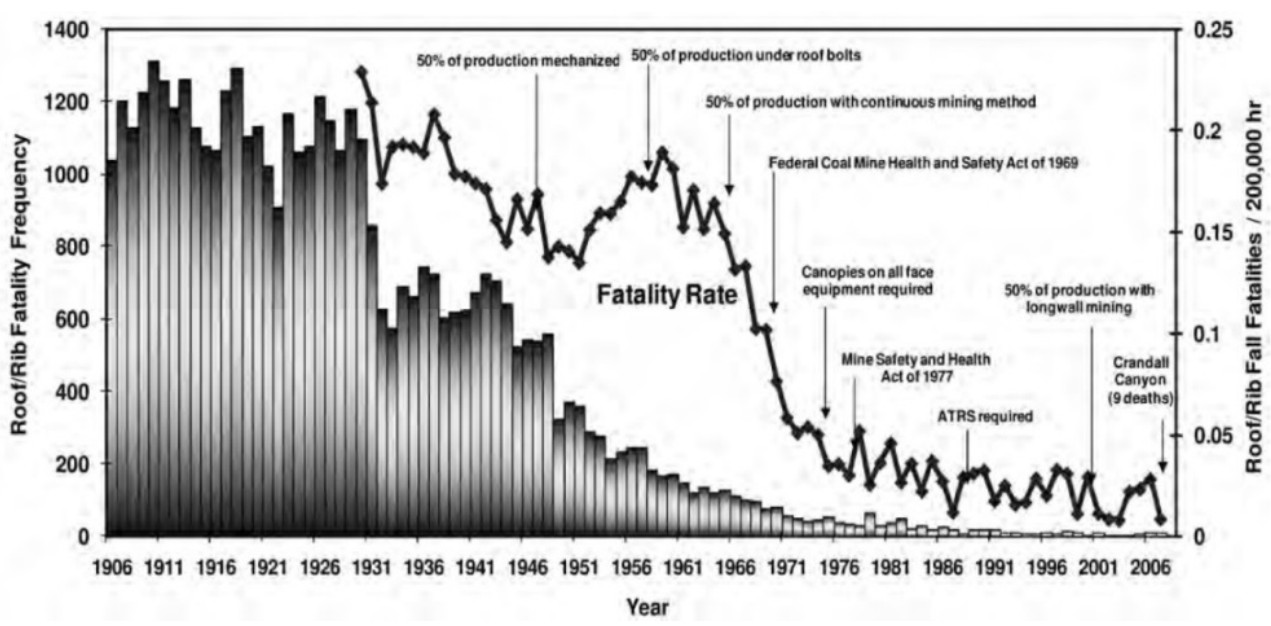

Fig. 1.1. Historical roof and rib fall fatality rates (Pappas and Mark 2012)

As a leading source of safety problems in underground coal mines, roof falls can cause fatality, injury and various non-injury problems. Fig. 1.1 shows a historical overview of the fatality rates for roof and rib falls. Please note how the fatality rate has slowed or plateaued in the past 30 years. However, from 1999 through 2008, roof and rib falls in U.S. underground coal mines still caused 75 fatalities, 5,941 injuries and experienced 13,774 non-injury roof falls (Pappas and Mark 2012). According to the U.S. Department of Labor's Mine Safety and Health Administration (US-DOL 2014), 377 and 265 miners were injured in roof and rib falls in 2012 and 2013, respectively. These injury and non-injury roof falls usually lead to time and economic loss in the form of damaged equipment, stopped production or disturbed ventilation.

Researchers have made significant efforts to investigate and prevent roof fall through various methods and decades of studies. They have recognized that contributing factors such as: mechanical properties of rock, topography, in-situ stress, and mine layout can be critical under certain conditions in roof fall formation. These studies also contain guidance for mine design and reinforcement design for specific situations (Aggson 1979; Su and Peng 1987; Wang and Peng 1996; Barron and Baydusa 1999; Gadde and Peng 2005; Esterhuizen et al. 2008; Ray 2009; Esterhuizen and Bajpayee 2012; Gao and Stead 2013).

However, field observation shows that the formation of roof falls is often a time-dependent process. As noted by Dolinar et al. (2000), the commencement of roof falls varies from a few days to several weeks after an opening was mined. Another study by Ray (2009), based on field observation of a specific mine, found that more than fifty percent of roof falls had stand-up times between 1 to 2 years, whereas $12 \%$ of roof falls had stand-up times of less than 2 months. However, previous studies rarely considered the effect 
of time on roof falls. Therefore, it becomes necessary to study the roof behaviors from a time-dependent point of view.

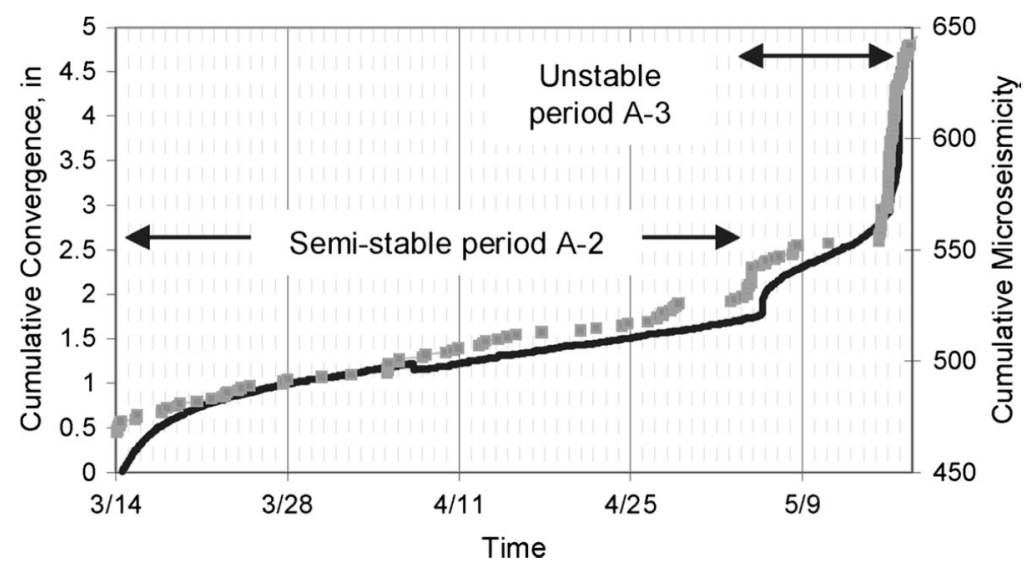

Fig. 1.2. Comparison between roof convergence (line), micro-seismic event frequency (dot) and local stability conditions (Iannacchione et al. 2004)

The time-dependent behavior of mine roof, when plotted as cumulative convergence versus time, has a similar shape as a creep curve with three stages. The deformation velocity usually decreases quickly at first, then keeps constant for a long period and finally accelerates to failure when the deformation exceeds a certain threshold value. This accelerating convergence is often a sign of pending roof instability.

At first glance, studying this time-dependent roof convergence behavior from a point of view focused on creep seems like the most appealing option. However, this measured convergence data does not merely represent the creep behavior of roof rocks (Malan et al. 1997). Aside from the creep of intact rock, the measured deformation usually includes elastic and plastic deformation, and even separation of bedding planes. In addition, the measured deformation includes the structural response of the roof. For example, cutter roof failure is the common roof failure mode in underground coal mines. It usually initiates at one entry corner and gradually propagates upward with time. The propagating failure will change the immediate roof from a clamped beam into a cantilever beam which, in turn, increases the deformation of the immediate roof. Therefore, the roof convergence cannot exactly represent just the time-dependent behavior of roof. The time-dependent behavior of overall roof involves various phenomena, including time-dependent deformation of intact and failed roof rocks, strength deterioration, time-dependent failure propagation and structural response of the roof rocks. In addition, the presence of bedding planes and laminations can affect the roof stability in the form of decrease in rock mass strength and change in the failure mode. This complexity of failure demonstrates the necessity of a comprehensive study on time-dependent roof behavior in order to fully understand the observed phenomena.

\subsection{RESEARCH QUESTON AND OBJECTIVE}

Fig. 1.3 demonstrates the conceptual process of cutter roof. Due to the directional horizontal stress, failure first initiates at one entry corner during entry development, which alters the stress distribution within roof. Next, the failed rock further deforms in the post-failure region, and the failure gradually propagates upward with time. These behaviors interact with one another during this process. As such, the study on the 
time-dependent deformation and failure process of roof in underground coal mines involves various rock mechanics phenomena observable in the failure process.

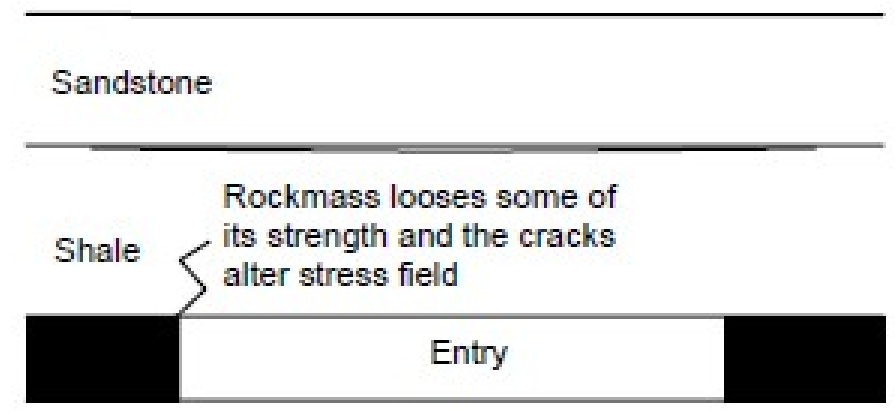

(a)

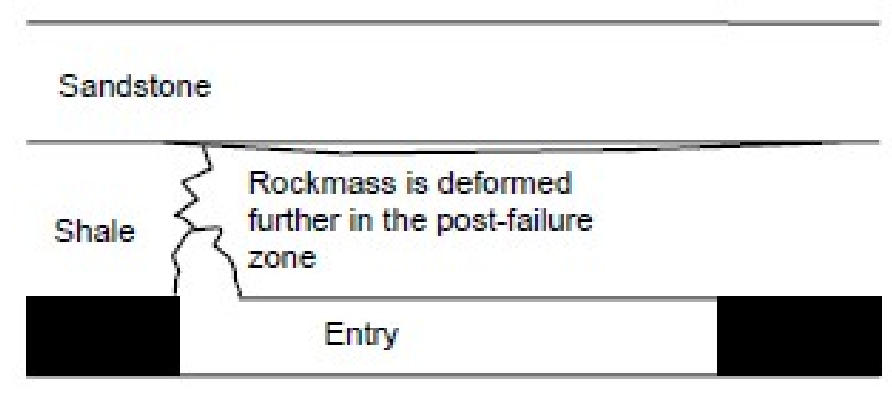

(b)

Fig. 1.3. Cutter failure process in underground coal mines (Gadde and Peng 2005)

First, the time-dependent deformation or creep of intact roof rocks is the first of several sources for roof convergence. As creep is used to describe the time-dependent deformation with constant external load, it is often the first factor considered when investigating the time-dependent roof convergence. However, limited data is available for the time-dependent properties of coal measure rocks. In addition, failed roof rocks continue to move in the post-failure region where their time-dependent parameters are generally unknown. The difference in time-dependent property for intact and failed rock is significant. However, a creep test (the primary test method to determine time-dependent parameters) is difficult to perform on failed rock. As a result, the parameters for the time-dependent property of failed rock is not currently available.

Second, the formation of roof falls always involves rock failure. The stress redistribution during development could induce roof falls immediately or just initiate a time-dependent failure process. For the latter case, the failure could gradually propagate with time and finally result in a roof fall. This timedependent failure propagation can be the result of stress redistribution and/or strength deterioration. Due to strain-softening and the significant stress relaxation of failed rock, the rock stress will be redistributed from the failure zone to the surrounding solid rock, promoting the failure propagation; at the same time, the failure propagation will further alter the stress distribution. Therefore, the stress distribution and failure propagation are interacting over time. Also, rock strength can deteriorate due to environmental influence and viscous behavior. This research excludes the environmental influence. If rock strength gradually 
decreases with accumulated viscous strain, failure could propagate with time, even without any change in the stress distribution.

Third, a strain-softening/hardening model can represent the post-failure behavior of rocks; however, it is not often used for time-dependent study. Strain-softening/hardening models are widely used to simulate the progressive failure in time-independent studies; however, time-dependent studies rarely consider strainsoftening/hardening behavior. In reality, the time-dependent behavior and strain-softening behavior can both occur and interact with one another in the post-failure region. Therefore, the combination of a strainsoftening model and a time-dependent model is necessary for the study of the true time-dependent formation of roof falls.

Fourth, other factors, which have been recognized as making a roof prone to failure, can also affect the roof failure process from a time-dependent point of view. Directional horizontal stress, mine layout, and reinforcement affect the stress distribution and further affect the roof stability, which have been recognized through theoretical analysis, numerical simulation and field observation. A study focusing on roof falls from a time-dependent point of view should investigate these factors' influence.

Finally, the failure process of bedded roof, common in underground coal mines, is different from that of massive roof. The presence of bedding planes decreases rock mass strength and induces anisotropy. The bedding planes reduce the integrity of the roof by dividing the roof into beams, and as a result, they change the roof failure mode completely. Therefore, bedding planes should be explicitly included bedding planes when investigating the time-dependent roof deformation and failure process.

These analyses have helped to form the following research problems as the basis for this thesis:

1) find an alternative test method which can determine the time-dependent property of both intact and failed rocks;

2) determine the observed time-dependent behaviors in the pre-failure and post-failure region from the new laboratory test;

3) propose a constitute model, capable of representing all the observed time-dependent and timeindependent rock behaviors, for numerical simulation;

4) conduct simulations of the time-dependent deformation and failure process for massive roof with various factors for the purpose of investigating the time-dependent stress distribution and failure propagation, and for studying the influence of various factors on the failure process;

5) conduct simulations of the time-dependent deformation and failure process of bedded roof to help understand the influence of bedding planes on the time-dependent failure process.

Resolving these research problems requires a large amount of laboratory tests, theoretical analysis, programming and numerical simulations. First, this study must propose a test method which can help study the time-dependent behaviors of intact and failed rocks, verifiable through laboratory tests. This test method and other laboratory test methods will combine to determine the time-dependent property for rocks in the pre-failure and post-failure region. Compression tests with different strain rates and/or creep tests will investigate the strength deterioration. In addition, together with a strain-softening model and viscous model, one constitutive model is expected to describe the strain-softening behavior and viscous behavior. This expectation originates from the results of time-dependent laboratory tests in the post-failure region. Finally, 
this study will program the constitutive model into numerical software for simulation purpose. The simulation will showcase two situations: massive roof and bedded roof- with various factors to study the time-dependent deformation and failure.

In summary, the objective of this research is to investigate the time-dependent behaviors of intact and failed rocks and the associated strength deterioration with an alternative test method in laboratory, as well as to build and code a constitutive model to simulate the underground coal mine roof under different conditions. A comprehensive understanding of the time-dependent formation of roof falls is the expected result of this research.

\subsection{OUTLINE OF THE DISSERTATION}

The final purpose of this study is to investigate the time-dependent deformation and associated failure of roof in underground mines through laboratory test and numerical simulation. To achieve this goal, the following seven chapters contain the research work of this thesis.

The second chapter contains the literature review. The review concerned topics related to the research questions such as the time-dependent behavior of rocks, rock failure and strength degradation, strainsoftening behavior, bedded rock, and roof falls to determine the current research status in these research areas. The summary afterwards discusses the research methods that can be adopted in this study and the drawbacks of previous studies that need to be improved.

The third chapter contains the study of rock time-dependent property using a relaxation test (the primary test method used in this study). This involved using Burgers model to derive a relaxation equation, as well as proposing a procedure to determine the viscous parameters from relaxation test results. Laboratory creep test and relaxation test were then conducted on various specimens and the viscous parameters determined from creep test and relaxation test were compared. The good agreement in the viscous parameters determined from different test methods demonstrated that relaxation test can be used to study the timedependent property of rocks.

The fourth chapter discusses the influence of the variation in time-dependent properties and strength degradation on time-dependent roof failure. The variation in the time-dependent property and strength degradation are the two important time-dependent behaviors of rocks. Laboratory relaxation tests in published literature assisted in investigating the influence of the variation in the time-dependent property on the roof behavior. During numerical simulations, a FISH function was written to control the varying viscous parameters. The simulation results show pronounced influence and demonstrate the mechanism for

the failure propagation induced by the varying viscous parameters. In addition, Burgers model associated Mohr-Coulomb failure criterion in 3DEC was used in the simulation of a creep test on a rock specimen. The specimen continued deforming without failure, indicating that the built-in models did not consider the strength degradation. The concept of effective viscous strain was introduced into 3DEC though a FISH function. The rock deteriorated with accumulated effective viscous strain. The simulation of a mine model shows the time-dependent failure propagation with rock deterioration. 
The fifth chapter is the laboratory study on the time-dependent behavior of intact and failed rock under unconfined condition with relaxation test. Laboratory relaxation test was conducted on sandstone specimens. Radial strain gauge was used for successfully capturing the strain-softening process. Each specimen had one relaxation test conducted in the pre-failure region and the other conducted in the postfailure region. The study observed smooth relaxation behavior in the pre-failure region and step-wise relaxation in the post-failure region. Overall, the failed specimens relaxed more stress than the intact ones. The viscous parameters determined from the post-failure regions showed ignorable difference, regardless of the failure condition. Therefore, the viscous parameters of failed shale, sandyshale, and limy shale specimen tested in the residual stage in Chapter 3 were determined to represent the viscous property of the failed coal measure rocks.

The sixth chapter contains the development of a viscoelastic-strain-softening model. The important time-independent and time-dependent behaviors observed in the literature and laboratory tests were summarized and a new constitutive model that can represent all these behaviors was proposed. The strainsoftening model represents the time-independent behavior and Burgers model describe the time-dependent behavior. The new model also included the variation in the time-dependent property and the strength degradation induced by viscous behavior. This chapter also presents the implementation and programming of the constitutive model into 3DEC as a user-defined model, as well as the procedure to obtain the model parameters and model calibration. Finally, the model was verified by simulating various laboratory tests.

The seventh and eighth chapters contain the time-dependent study of massive and bedded roof in underground coal mines. For the massive roof, the new model was used to simulate the time-dependent response of the mine roof with directional horizontal stress to demonstrate the time-dependent failure process. This included a numerical investigation into the influence of various factors, including directional horizontal stress and step-wise excavation affecting the stress path on the roof behavior. For the bedded roof, the study proposed a simplified two-dimensional viscoelastic beam model to study the influence of bedding planes on the time-dependent stress distribution. Factors investigated in this section included different beam thicknesses, horizontal stresses and bedding plane strengths. A three-dimensional model with the new constitutive model was used to study the time-dependent behavior of bedded mine roof with directional horizontal stress.

Finally, the closing summary includes the overall conclusions of the research and possibilities for future study. 


\section{CHAPTER 2 \\ LITERATURE REVIEW}

\subsection{INTRODUCTION}

The research on time-dependent deformation and failure of roof in underground coalmines involves various rock mechanics processes. This chapter discusses and summarizes previous studies on these topics.

For decades, studies have investigated roof falls as a leading cause of injuries in underground coal mines from a time-independent point of view. Various methods such as theoretical analysis, physical model, numerical simulation, and field observation, have researched the failure mechanism, influence factor and prevention. These studies have drawn important conclusions in relation to this topic. These studies are still applicable to the time-dependent study and are thus reviewed first.

In addition, the time-dependent deformation and failure of roof always involve creep of roof rocks. This is usually the first factor to consider when studying the time-dependent roof deformation because creep is the term used to describe the time-dependent deformation with constant external load. It is a common behavior of rocks; as such, many have conducted comprehensive studies on rock creep for different purposes. Various research efforts have involved the performing of a significant amount of laboratory creep tests; as such, studies have proposed different constitutive models, including rheological model, empirical model, visco-plastic model, damage model, and micromechanical model, to represent the creep behavior of rocks. The second section contains a comprehensive review of these studies.

Also, the time-dependent deformation and failure of roof always involve rock failure. The timedependent failure process can be the result of stress redistribution and/or time-dependent strength. Due to environmental influence and viscous deformation, rock strength gradually deteriorates over time. There are two ways to represent the time-dependent strength: (1) time-dependent strength where the strength decreases with time and therefore the strength is directly related to time, or (2) strength deterioration where the strength decreases with viscous deformation, and the strength is indirectly related with time. In addition, these studies contain various laboratory test methods, creep test, and compression tests with different strain rates, to study the time-related strength. The third section reviews the research in this area in detail.

In addition, behaviors of failed rock are important in the time-dependent deformation and failure process of mine roof. Failed rock still exists in mine roof and deforms in the post-failure zone. There are two kinds of behaviors observed in this process: strain-softening behavior and time-dependent behavior. These can both occur and interact with each other for failed rock. However, the time-dependent studies rarely consider the strain-softening behavior. The fourth section reviews this information.

Finally, the failure process of bedded roof is different from that of massive roof. Bedding plane is common in the roof of underground coalmines and it has various influences on the time-dependent deformation and failure of roof. First, the presence of bedding planes decreases rock mass strength and therefore induces more failure in the roof. Second, the bedding planes reduce the integrity of the roof by 
dividing the roof into beams and as a result, completely change the roof failure mode. Finally, creep behavior can also occur along these bedding planes. This section reviews previous studies on timeindependent and time-dependent of discontinuities.

\subsection{ROOF FALL}

Due to its prevalence in underground coalmines, researchers have investigated roof falls for decades using different methods. These studies have drawn some important conclusions about the mechanism, influencing factors, and prevention.

Aggson (1979) summarized the roof failure mechanisms under different conditions. For a massive roof, the most probable failure mode is an arch-shaped shear failure in the roof with high ratio of horizontal stress to vertical stress. The failure starts near the corner of roof and pillar and the angle of propagation of the failure surface depends on the ratio, where lower angles relative to the horizontal usually associate with higher ratio of horizontal stress to vertical stress. With low ratio, the angle of the failure surface will rotate towards the vertical stress; when the failure surface is vertical, cutter roof is formed. In addition, if the horizontal stress is considerably less than the vertical stress, the most probable failure mode is a vertical tension crack in the center of the span, where the maximum tension is gained. In contrast, if the entry is excavated with laminated roof, the failure mode will be quite different. The immediate roof will deflect downward to the entry and fail first. As a result, the second roof bed loses its lateral confinement and is free to fail into the entry in the same manner. This failure process of the laminated roof layers continues until a thicker layer is reached or the failing roof establishes a stable geometry.

Su and Peng (1987) examined the intrinsic mechanisms of cutter roof failures by means of field investigation, laboratory testing, underground instrumentation and numerical modeling. They examined various factors including high vertical stress, excess horizontal stress, relative stiffness between coal and its immediate roof, and large topographic relief, through parametric study with finite element analysis. As such, they drew comprehensive conclusions from this study, and summarized as follows.

The study on in-situ stress shows that the larger the difference between the horizontal stress and the vertical stress, the more the failure plane deviates from the vertical; whereas if no excessive horizontal stress is present, or if the maximum horizontal stress perpendicular to the entry is close to the overburden likely to propagate vertically at the entry corners to the second layer. In regard to the relative direction between maximum horizontal stress and mining direction, cutter roof failures will occur in the crosscuts if the entries are parallel to the maximum horizontal principal stress in an excess horizontal stress field with high differential stress. Conversely, if the entries are driven parallel to the minimum horizontal stress, cutter roof failures will occur in entries.

In addition, the first immediate roof is more likely to occur near the rib-roof intersections when the mine is under thick overburden and no excess horizontal is present. This is also applicable when the coal pillars become stiffer. On the other hand, under an excess horizontal stress field with high differential stress, the horizontal stress will arch deeper into the roof as the first immediate roof layer becomes softer. As a result, cutter roof is more likely to occur if the first immediate roof layer is stiffer. High vertical stress coupled with a stiffer coal is the dominant factor in the formation of cutter roof. 
It is also notable that when the coal is stronger than the immediate roof, cutter roof is likely to occur under thicker overburden if no excessive horizontal stress is present. However, with the presence of a directional high horizontal stress field, cutter roof will most likely occur under thinner overburden and the number of cutter roof occurrences under thicker overburden will be greatly reduced.

Finally, high vertical stress is the dominant factor controlling the behavior of the immediate roof at the entry corner. However, the magnitude and direction of the in-situ horizontal stress field actually control the location and nature of cutter roof occurrences in coalmine entries. Under high vertical stress, coal pillars with higher stiffness tend to increase the likelihood of cutter roof failure at the entry corners. On the other hand, under a directional high horizontal stress field a stiffer immediate roof layer tends to collect higher stresses and thus increases the likelihood of cutter roof occurrences. Large topographic relief on the surface exerts significant influence on the occurrence of cutter roof in coalmine entries. With uniformly sloping surface topography, cutter roof tends to occur at the upper right corner of a coalmine entry on the lower overburden side.

Wang and Stankus (1998) demonstrated the importance of the high horizontal stress field in roof stability with finite element analysis; they also provided a guideline for orientating the panel in a favorable direction to avoid high horizontal stress concentration. The entry roof will be in good condition if the mining direction is within the range of $\mathrm{N} 58^{\circ} \mathrm{W}$ to $\mathrm{S} 42^{\circ} \mathrm{W}$ and $\mathrm{N} 42^{\circ} \mathrm{E}$ to $\mathrm{S} 58^{\circ} \mathrm{E}$.

Barron and Baydusa (1999) developed a theory for cutter roof failure in coalmines based on the limit equilibrium stability analysis of a truncated roof wedge. The result demonstrates that the key parameters affecting the likelihood of cutter roof failure are the rock strength, the vertical stress and the horizontal to vertical stress ratio.

Gadde and Peng (2004) investigated the effect of in-situ stress on the stability of coalmine entries and intersections with ABAQUS. Factors investigated in this study include the in-situ maximum horizontal stress, the ratio of in-situ maximum horizontal to vertical stress and the ratio of in-situ maximum horizontal to minimum horizontal stress. The result indicates that, in general, entries orientated in the direction of maximum horizontal stress are in the best condition; those oriented at $90^{\circ}$ have the least stability, and the maximum and minimum stability for intersections are seen at $0^{\circ}$ or $90^{\circ}$ and $45^{\circ}$, respectively. Also, the effect of in-situ maximum horizontal stress to vertical stress ratio on an entry or intersection stability follows the shape of a lognormal distribution curve and the entry stability also depends on the value of in-situ maximum horizontal to minimum horizontal stress ratio.

Gadde and Peng (2005) proposed the use of strain-softening model to simulate the cutter initiation and propagation process. The result demonstrates the possibility of using strain-softening model with implementation of cutting sequence to capture spatial distribution of cutters close to field observation at an Illinois mine. Ray (2009) further adopted this method for the purpose of checking the influence of cutting sequence over cutter formation. As the mining development or cutting sequence affects the sequence of stress redistribution for a specific cross-section, the study of cutting sequence investigates the influence of stress path on cutter formation. The modeling results show that, among all the factors that could affect cutter distribution in a panel, it is the cutting sequence and the spatial variability of rock strength that play the 
overriding roles. In addition, it is the relative magnitude of the in-situ stresses as compared to the rock mass strength that determine the severity of the cutter problem. In addition, the model results indicate that the severity and spatial distribution of cutters in a panel primarily depend on the two in-situ stress ratio: the ratio of maximum horizontal stress to vertical stress and the ratio of maximum horizontal stress to minimum horizontal stress ratio. From this study, it is also notable that realistic simulation of the mine development step-by-step is very crucial in capturing the progressive failure process. Sherizadeh and Kulatilake (2016) also used strain-softening model to study the roof stability in a roof and pillar coalmine with 3DEC, where bedding planes were included into the immediate roof. The result demonstrates that the progressive failure of roof can be successfully simulated with strain-softening model and shows that the bedding plane plays a major role on the geo-mechanical behavior of roof in underground excavations.

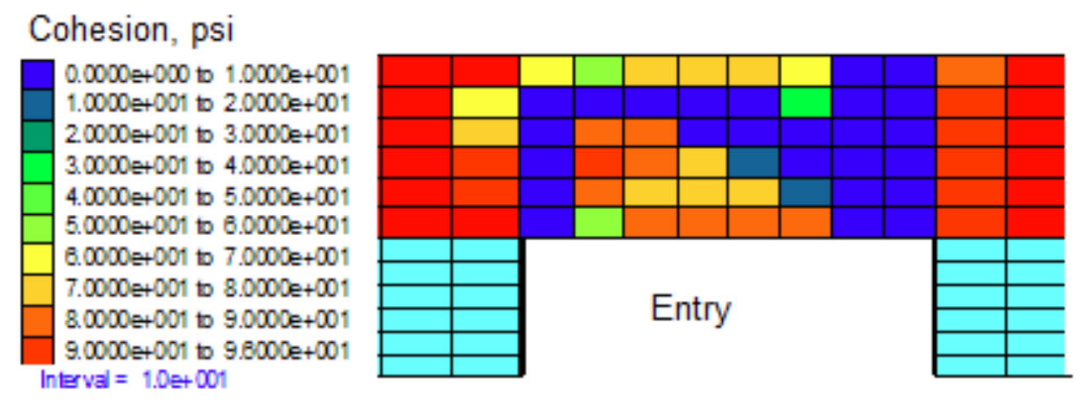

Fig. 2.1. Distribution of cohesion showing cutter development (Ray 2009)

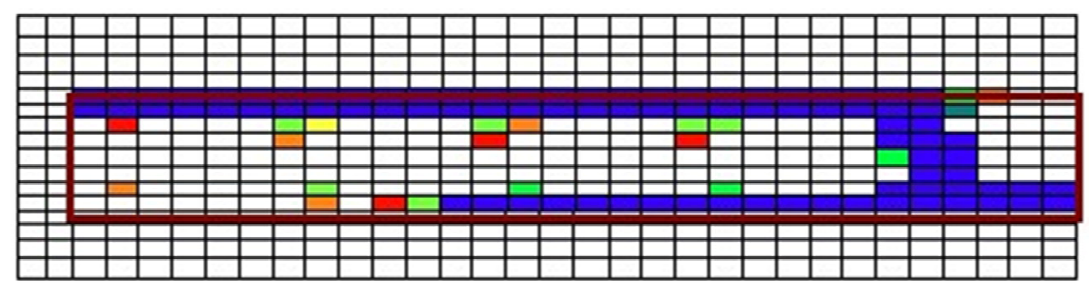

Fig. 2.2. Distribution of cutter in immediate roof (Ray 2009)

Roof fall surveillance data from NIOSH indicated that a large percentage of roof falls occurred in mines with weak roof (Molinda and Mark 2010), where the weak roof was defined as a roof with a CMRR $\leq 40$ or roof rock with an unconfined compressive strength $\leqslant 3500$ psi (Mark et al. 2004). Based on the field observations, Molinda and Mark (2010) summarized the common roof fall types related to weak roof, including stackrock delamination, roof deflects, cutter roof, spalling roof, sandstone bodies in the roof, and roof sag. They believed that recognition of geological structures and their behavior under stress is an important first step in safe roof support practice.

Esterhuizen et al. (2008) studied horizontal stress effects on roof stability in US limestone mines. The field observations show various roof behaviors related to horizontal stress. Once large oval-shaped roof falls occur, the falls can gradually extend in the lateral direction, perpendicular to the direction of the maximum horizontal stress, across multiple tens of meters of a mined area. The numerical simulation results show that the bedding planes in the immediate roof can exacerbate the depth and extent of rock failure in the roof and roof stability is further degraded by increased deflection and separation of the bedded roof. 
The models also demonstrate the great advantage in aligning the pillar layout parallel to the direction of maximum horizontal stress and offsetting cross-cuts so that lateral growth of roof failure is restricted.
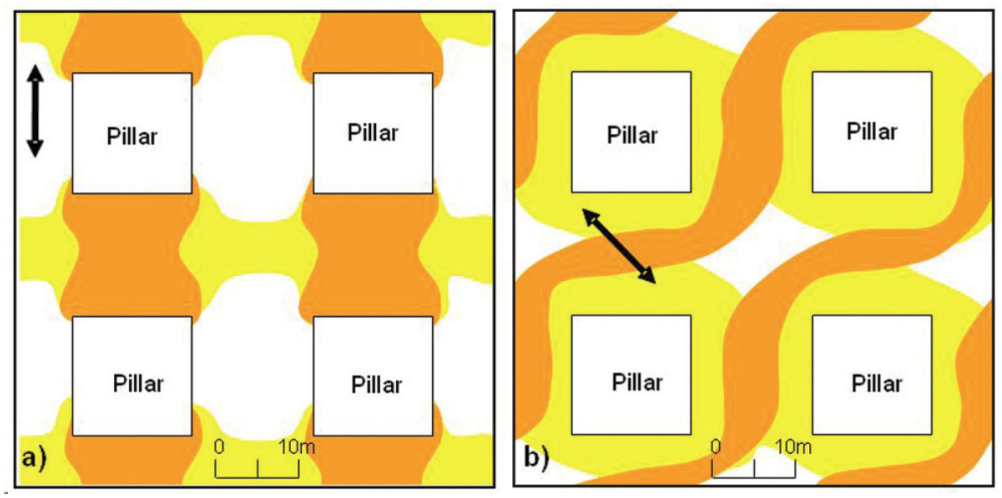

Fig. 2.3. Plan view showing effect of a change in the orientation of the maximum horizontal stress potential roof failure in a room and pillar layout consisting of square pillars.

Darker shading indicates initial failure and lighter shading indicates potential failure growth. The arrow indicates the direction of maximum horizontal stress (Esterhuizen et al. 2008)
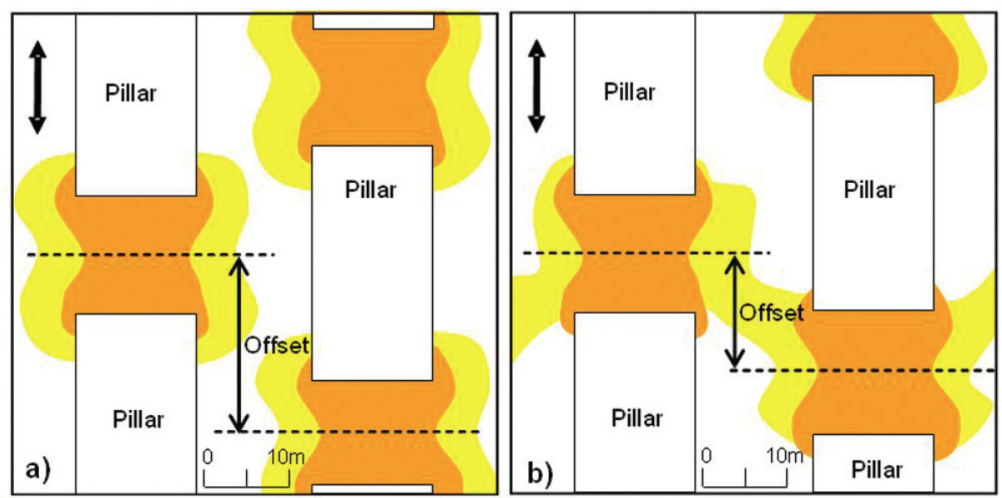

Fig. 2.4. Plan view showing effects of a change in the pillar offset on potential failure in a room and pillar layout consisting of rectangular pillars.

Darker shading indicates initial failure and lighter shading indicates potential failure growth. The arrow indicates the direction of maximum horizontal stress (Esterhuizen et al. 2008)

Esterhuizen and Bajpayee (2012) numerically simulated bedded roof with high horizontal stress where stepwise increments in horizontal stress were applied from one side of the model. The comparison of potential failure between the massive roof and the bedded roof demonstrates that the presence of laminations leads to a much greater zone of potential failure than the massive roof. The study on sensitivity to changes in interface properties shows that, for low strength/low stiffness interfaces, the failure mode tends to more closely resemble cutter failure and occurs at the excavation corners, progressing nearly vertically. As the interface friction and stiffness increases and approaches the rock strength, the failure tends to become more inclined and develops an inclined shear plane. Failure at the center of the roof span occurs more frequently when the rock strength is relatively higher, and the interface friction was lower than the base case analyses. 


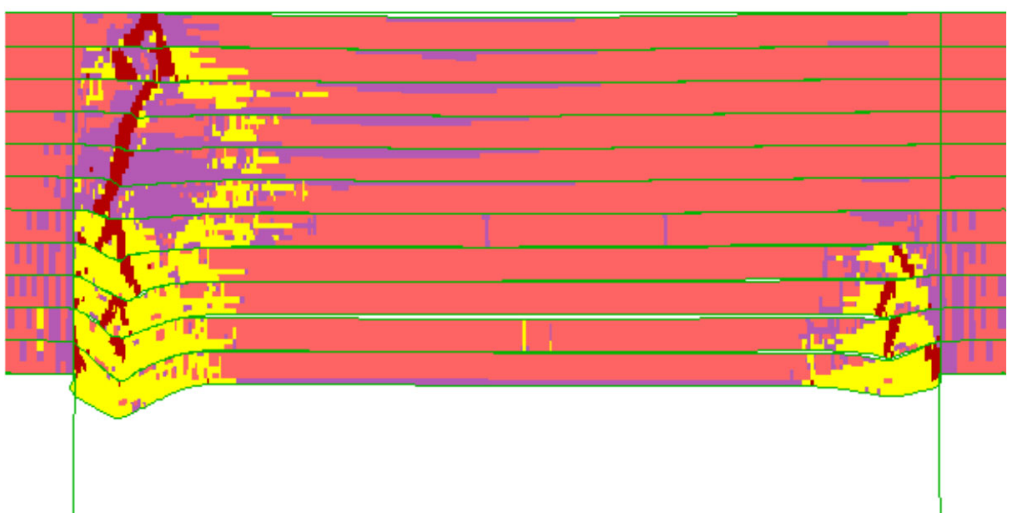

(a) Bedding planes with low strength and stiffness and $10 \mathrm{~cm}$-thickness

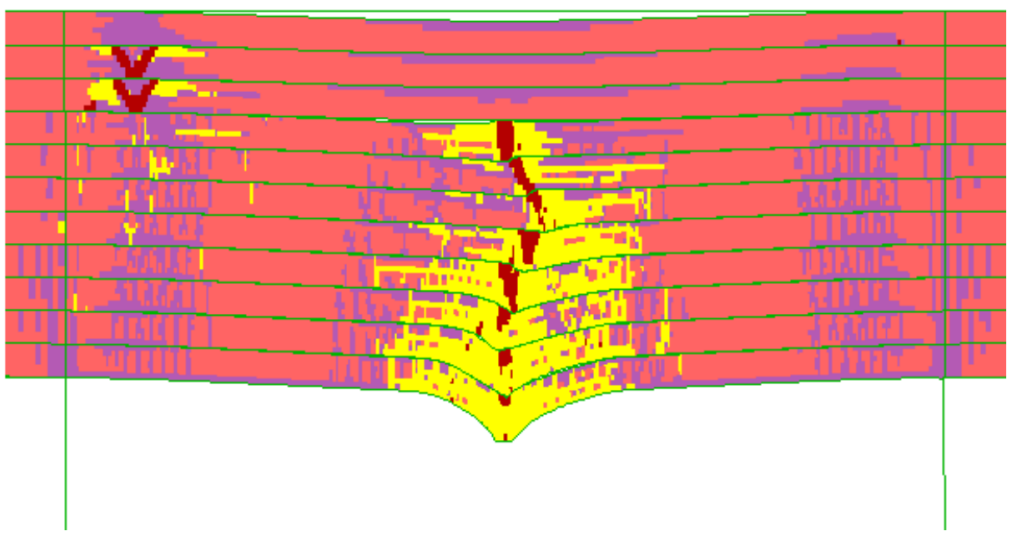

(b) Bedding planes with high strength and stiffness and 10cm-thickness

Fig. 2.5. Numerical simulation of bedded roof failure with different interface properties (Esterhuizen and Bajpayee 2012)

Coggan et al. (2012) used various simulation methods for the purpose of investigating the effects of weak immediate roof lithology on coal mine roadway stability. First, they studied the influence of horizontal stress direction, and the results show that tunnels driven perpendicular to a high horizontal stress direction suffer greater deformation and increased failure zones when compared to tunnels driven in a stress-parallel direction. In addition, tunnels driven at an angle to the in-situ stress field suffer asymmetrical deformation and failure; three-dimensional modelling is necessary to capture three-dimensional nature of the stress redistribution around a tunnel face, particularly when the maximum horizontal stress is aligned at angle to the tunnel driven direction. Next, they simulated the immediate mudstone roof with various thicknesses, and the result demonstrates that the thickness of weak mudstone in the immediate roof of the tunnel controlled the extent of the modelled failure zone. 

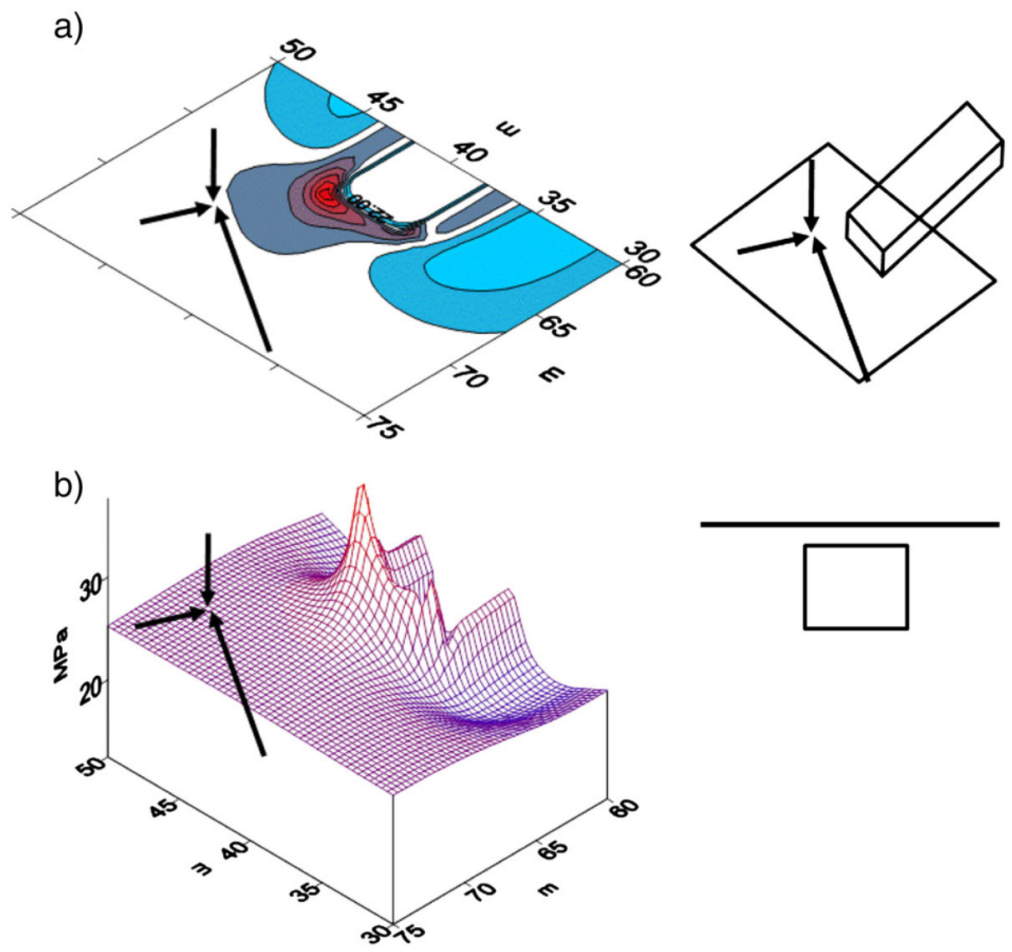

Fig. 2.6. Maximum principal stress distribution on a plane $0.25 \mathrm{~m}$ into immediate roof of a tunnel where the maximum horizontal stress is aligned at 65 degrees to tunnel drivage direction (a) modelled principal stress on plane $0.25 \mathrm{~m}$ into tunnel roof, (b) carpet plot, stress trajectories represent the relative magnitude and orientation of the in-situ stress (Coggan et al. 2012)

Gao and Stead (2013) simulated the formation of cutter roof failure with 3DEC and PFC3D with respect to the orientation of major horizontal stress and the forward of excavation. Gao et al. (2014) simulated roof shear failure in coalmine roadways with UDEC. Voronoi tessellation meshed the model into randomly sized polygonal blocks. The study used a built-in FISH function for UDEC to gradually release the stresses applied on the roadway surface in a total of 10 stages to simulate a more realistic model excavation with continuous mining machine, rather than a sudden excavation of the roadway. In such a way, the formation and development of shear failure or crack can be visualized at each stage. Shear cracks initiate at the ribroof corners where a stress concentration occurs and then propagate into the roof. The length of the shear cracks increases quickly with the gradual relief of applied stress. Nearly $90 \%$ of the total shear cracks occur when $90 \%$ of the total stress is released, which indicates that the roof is heavily fractured in shear before the roadway is fully excavated. However, the initiation and development of tensile cracks are different from shear cracks. Isolated tension cracks initiated within the middle roof when $50 \%$ of the applied stress is released and further increased with released stress. These discrete tension cracks start to interact when the released stress is $90 \%$. Beyond this point, the lengths of tension cracks increase quickly. To determine this, the study used a FISH function to track the number and length of failed UDEC trigon contacts in shear or tension within the area of interest. 


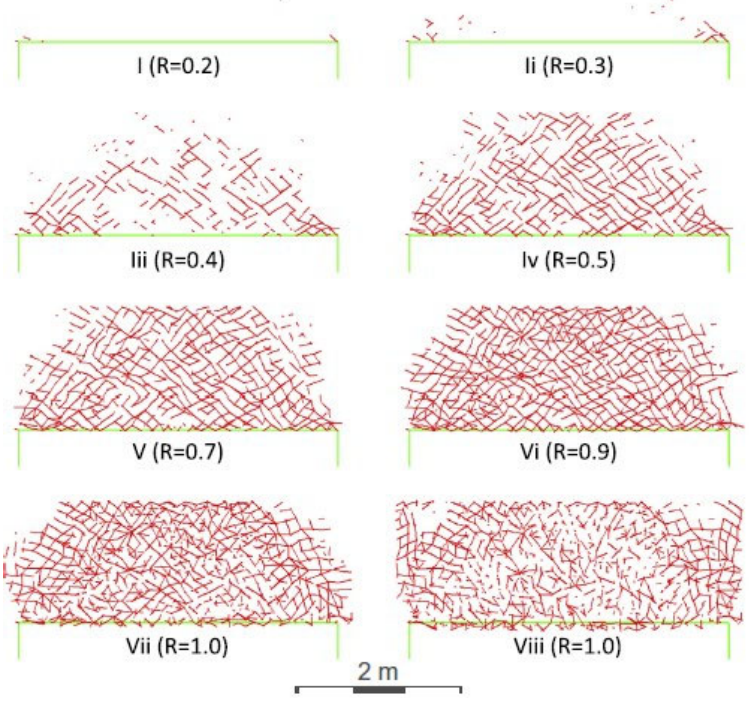

Fig. 2.7. Development of shear cracks in the roadway roof during stress release (Gao et al. 2014)

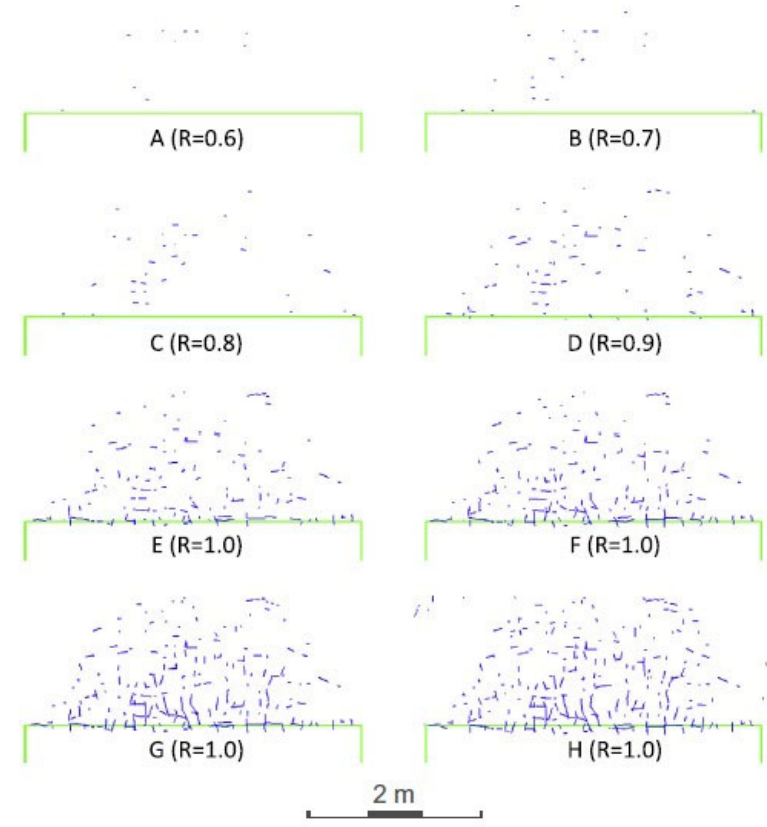

Fig. 2.8. Development of tension crack in the roadway roof during stress release (Gao et al. 2014) 


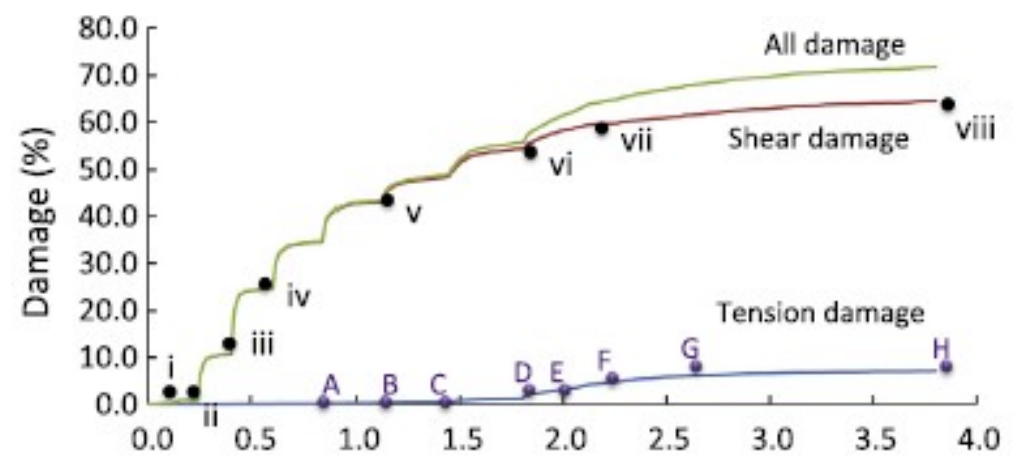

Fig. 2.9. Damage development in the roadway roof during stress release (Gao et al. 2014)

The study of these literatures emphasizes the various factors affecting the stability of underground coalmine roof such as the magnitude and direction of in-situ stress, stress path, roof rock property, and geological features. However, field observation shows that the formation of roof falls is a time-dependent process. As pointed by Dolinar et al. (2000), the commencement of roof falls varied from a few days to several weeks after an opening was mined. Also, Ray (2009) found that based on field observation of a specific mine in Illinoi, more than 50\% of roof falls had stand-up time between 1 to 2 years, whereas 12\% of roof falls had stand-up time of less than 2 months. In addition, Molinda and Mark (2010) considered cutter roof as a time-dependent process; while cutter roof often damages the roof at the working face, roof falls may not occur until months after mining when gutters have progressively severed the roof beam. Therefore, study on roof fall from a time-dependent point of view is ideal; however, there is no comprehensive study investigating the influence of time on time-dependent deformation and failure of underground coalmine roofs.

\subsection{TIME-DEPENDNT BEHAVIOR OF INTACT ROCK}

When considering the time-dependent behavior of mine roof, a convergence curve is the common representation of the relation between cumulative roof convergence and time. As previously mentioned, this convergence curve normally has three stages and is very similar to a creep curve. The deformation velocity usually decreases quickly at first, then keeps constant and possibly accelerates to final failure when the deformation exceeds a certain threshold value. The time-dependent deformation and failure process is very similar to the creep behavior of intact rock. As a result, the time-dependent roof displacement is a widely-used indicator for the time-dependent stability of mine roof (Iannacchione et al. 2004), and there are multiple studies focusing on determining the creep property of intact rock and finding a proper constitutive model to represent the observed creep behaviors.

Creep refers to continuously increasing strain with constant applied load. This means that, when a constant load is applied on rock, the rock will continue to deform. Different mechanisms, like timedependent micro-fracturing, recrystallization, and dislocations at grain boundaries, are responsible for the creep of rocks; each may play a major role for a specific condition and rock type. For brittle rock at room temperature, the time-dependent/sub-critical propagation and interaction of micro-cracks are the major reason for the creep of brittle rocks in room temperature, while other mechanisms are secondary (Scholz 1968; Schwartz and Kolluru 1981; Hagros et al. 2008). 


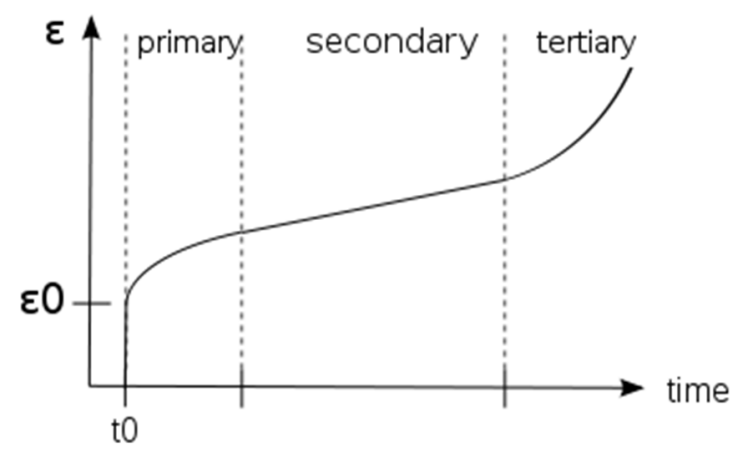

Fig. 2.10. Typical strain-time curve under creep condition

Laboratory creep test, either uniaxial or triaxial, is the normal method to investigate the creep behavior of intact rock. The test can obtain a creep curve that represents the relation between strain or strain rate and time when the applied load is kept constant. A typical creep curve consists of three stages: primary, secondary and tertiary, as shown in Fig. 2.10. Ito (1983) in his long-term creep experiment on granite beam over 24 years, concluded that granite could flow as viscous liquid without any yield stress. From then on, many studies used laboratory creep tests to determine the time-dependent properties for various purposes. They discovered that, for a specific rock type, various factors can affect the creep behavior, such as the deviatoric stress, water content, and temperature. After gathering the data from laboratory creep test, they determined a proper creep model and associated parameters to describe the creep behaviors. Various creep models, including rheological model, empirical model, viscoplastic model, and micro-mechanical model, are able to represent the rock creep behaviors in laboratory studies.

Wawersik (1972) studied time-dependent strength of Westerly granite, Nugget sandstone, and Tennessee marble using uniaxial compression test with various strain rates and uniaxial creep test. The test results indicate that there is a strong correlation between the quasi-static failure behavior and the tendency of rock to creep at ambient temperature. Rock which exhibits Class I failure behavior appears to be less prone to deform over time than Class II rocks.

Heap (2009) conducted conventional creep experiments on specimens of sandstone and basalt under triaxial stress conditions, which showed that creep strain rate is highly dependent on the applied differential stress; a reduction of only $10 \%$ in differential stress results in a decrease in creep strain rate of more than two orders of magnitude. The results demonstrated the possible existence of a critical damage threshold at the onset of the tertiary creep phase, regardless of the applied differential stress and the creep strain rate. The results of the following stress-stepping creep experiments demonstrated that the influence of an elevated temperature (from 20 to $75^{\circ} \mathrm{C}$ ) has a profound effect on the stress corrosion. For the same applied constant stress, creep strain rates increase by up to three orders of magnitude in sandstone. For basalt, however, creep strain rates are only modestly affected. This is likely due to the high quartz content of sandstone that has been shown previously to be greatly influenced by temperature in double-torsion experiments. 
Mishra and Verma (2015) conducted uniaxial and triaxial creep tests on laminated shale specimen, and the results show that the rocks under complex stress condition will deform with time and will transform into complete failure of the specimen.

Rather than studying the creep property alone, creep test results usually determine the proper creep model. The empirical model is a widely-used method to directly fit creep results from laboratory data. Hobbs (1970) performed uniaxial creep tests on cylindrical specimens of seven rocks, including siltstone, sandstone, shale, limestone and mudstone. The results indicate that an empirical equation can approximate the creep curve. After fitting the experiment results, Cruden (1971) found that an exponential law of transient creep plus a component of secondary creep is an inadequate description of some data while the power law of transient creep appears to fit all data satisfactorily without the addition of any component of secondary creep. There are the examples of empirical creep model and power, and logarithmic creep laws are such models.

In addition, another type of creep model to describe these behaviors is the widely-used rheological model. Rheological model normally consists of various springs and dashpots to describe the time-dependent behavior of rock. Kelvin model, Maxwell model, and Burgers model all belong to this category.

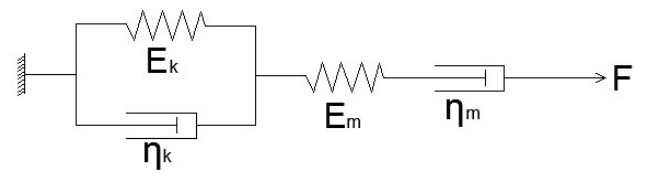

Fig. 2.11. Burgers model

Price (1964) conducted experiments to obtain information on the time-strain behavior of a number of coal measure rocks. The results indicated that the time-deflection relation of the bending tests could be directly interpretable with Burgers model while the results of compression tests showed a departure from Burgers model.

Li and Ghassemi (2012) performed a series of multi-stage tri-axial creep tests on Barneet, Haynesvile and Marcellus Shale for the purpose of assessing the contribution of creep to closure rate and conductivity loss of hydraulic fractures in gas shale. This study found that Power law and Burgers model can both describe the creep behaviors under the reported conditions.

Zhang et al. (2011) carried out tri-axial creep tests on glauberite, anhydrite and argillaceous rock salt. The results demonstrate that the creep property for the three kinds of rock salt is different under same condition and the strain-time relation shows good agreement with Burgers model.

In addition, the rheological model can include a viscoplastic part to describe the viscoplastic response. The above rheological models are viscoelastic model, which means that after unloading, the accumulated viscoelastic strain will recover with time. However, the time-dependent deformation is irrecoverable for some situations; in this case, the viscoplastic model is preferred. 


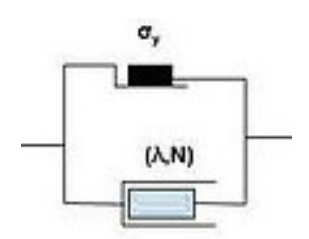

Fig. 2.12. Rheological viscoplastic part

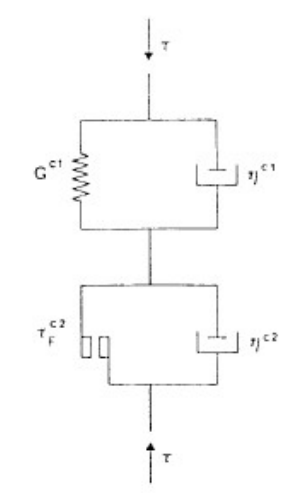

Fig. 2.13. Deviatoric rheological model

Gioda and Cividini (1981) and Gioda (1981) proposed a rheological model to describe the viscous behavior of rock. The model consists of two parts: one Kelvin unit for primary creep and one Bingham element for secondary and tertiary creep, which are considered to be viscoplastic. They used DruckerPrager yield condition to active the viscoplastic part. The viscosity coefficient for viscoplastic behavior decreases with increase in the applied shear stress and accumulated creep strain. As a result, the model can capture the three phases of creep behavior.

Sterpi and Gioda (2009) proposed a similar model to represent the behavior of squeezing rock during tunneling. The model represents primary creep with viscoelastic unit (Kelvin Unit) and secondary creep with viscoplastic unit. They also found that reducing the viscoplastic creep parameter with accumulated viscoplastic strains (second invariant of deviatoric viscoplastic strains) to account for the gradual mechanical damage can describe the process of tertiary creep. This makes the creep strain rate gradually increase transferring from secondary stage into tertiary stage.

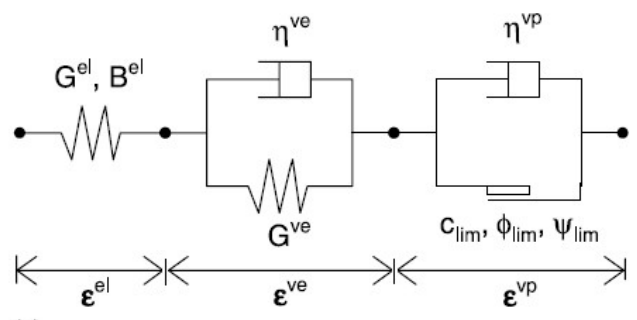

(a)

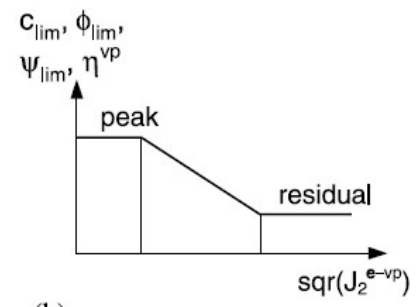

(b)

Fig. 2.14. A simplified rheological model

(a) model with associated parameters and (b) variation of the viscoplastic parameters with the $2^{\text {nd }}$ invariant of deviatoric viscoplastic strains (b) (Sterpi and Gioda 2009) 
Ottosen (1986) proposed a viscoelastic-viscoplastic model for rock salt, in which Burgers model represented the viscoelastic behavior while an additional viscoplastic elelment (including Bingham viscosity and a viscoplastic Kelvin element) described the viscoplastic behavior. The comparison between this model and power law model in fitting experimental creep data for rock salt shows that the new model demonstrates closer agreement with the creep data than the power law model.

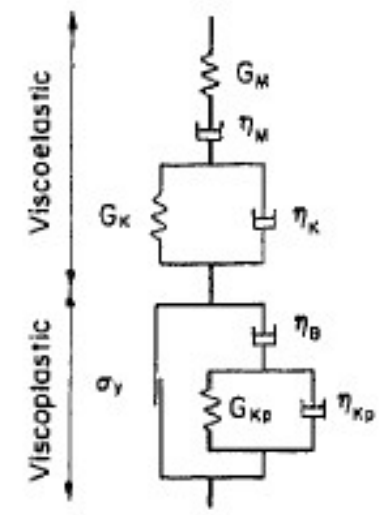

Fig. 2.15. Viscoelastic-viscoplastic model (Ottosen 1986)

In addition, either "over-stress" theory or damage theory can define the viscoplastic model. On one hand, if the model is based on "over-stress" theory (Perzyna 1966), the stress condition is allowed to be above the yield surface and the viscoplastic strain rate is proportional to distance of stress condition and the yield surface. Malan (1999) proposed an elasto-viscoplastic model to investigate the time-dependent closure data collected from a deep gold mine. The model used a time-dependent Mohr-Coulomb yield surface and considered cohesion as time-dependent to account for strength degradation with time. The rate of cohesion reduction was proportional to the excess stress above the residual target surface. This means that when the stress is closer to the strength value, the cohesion reduces more rapidly. The simulated results with FLAC show good agreement with the closure behavior of tabular excavations. Furthermore, Barla (2009), Debernardi and Barla (2009) and Barla et al. (2012) developed a new viscoplastic constitutive model, called SHELVIP (Stress Hardening Elastic Viscous Plastic), to describe the important features of the behavior observed in tunnels excavated in serve to very severe squeezing conditions. The model has one viscoplastic yield surface and one plastic yield surface, as shown in the following figure. When the stress condition is inside the viscoplastic yield surface, the deformation is purely elastic. Elasto-viscoplastic deformation occurs when the stress condition is located between the viscoplastic yield surface and the plastic yield surface. Here, the overstress concept allows that the stress goes above the yield surface and Drucker-Prager criterion is employed as the viscoplastic yield surface. If the stress condition is present on the plastic yield surface, elasto-viscoplastic-plastic deformation occurs and the stress condition cannot go beyond the plastic yield surface. With this model, the study simulated the time-dependent laboratory tests (Debernardi and Barla 2009) and advancing tunnels (Barla 2009; Barla et al. 2012) and observed good agreement. 


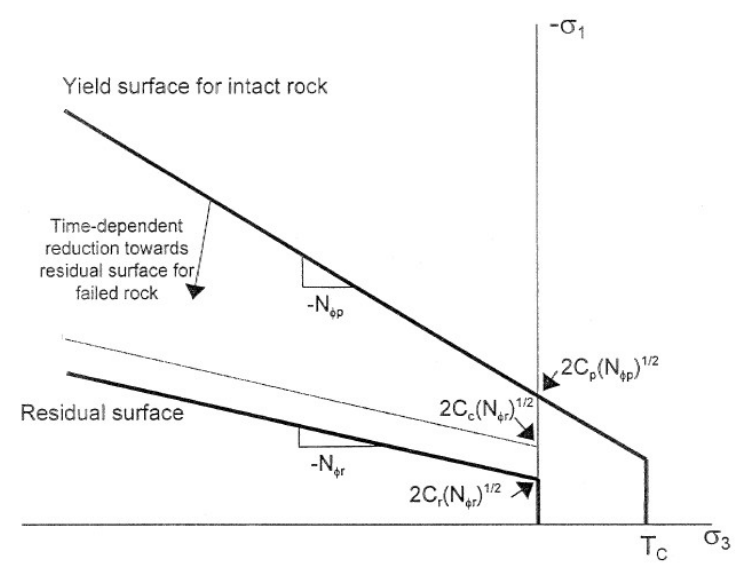

Fig. 2.16. The evolution of yield surface for intact rock to the eventual residual surface for failed rock (Itasca 2012)

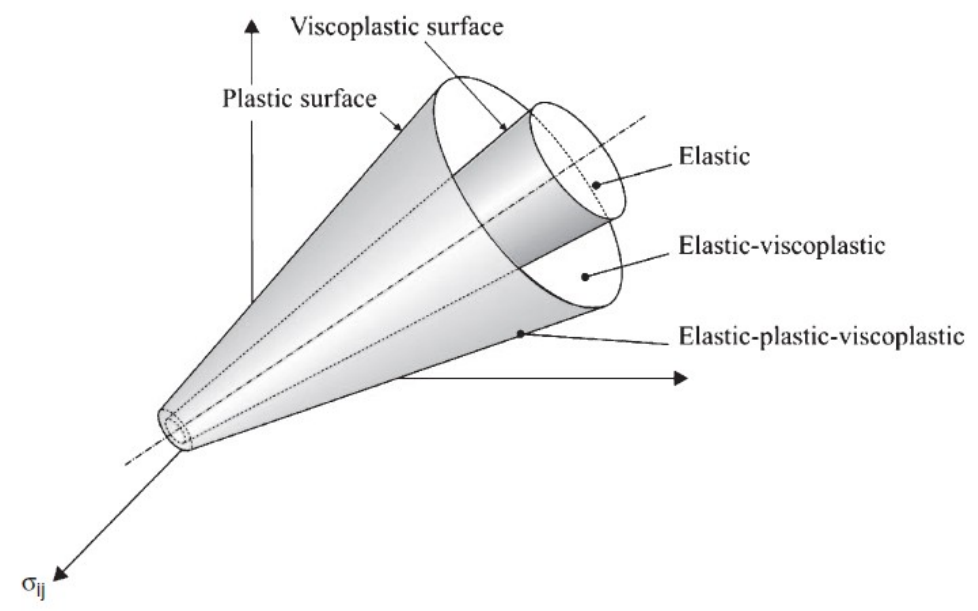

Fig. 2.17. Limit surfaces and stress fields in the principal stress plane for the stress hardening elasto-viscoplastic model (Itasca 2012)

On the other hand, if the time-dependent micro-fracturing is the primary mechanism for creep in brittle rocks, the accumulated creep strain deteriorates rocks with time (Lau and Chandler 2004) and can represent the evolution of damage with time. Introducing this evolving damage into elastic-plastic models can describe time-dependent behavior. Different damage parameters can represent the time-dependent deterioration and various methods, either damage mechanics (Dragon and Mroz 1979; Costin and Mecholsky 1983; Shao et al. 2003, 2006) or thermos-dynamics (Hamiel et al. 2004; Challamel et al. 2005; Pellet et al. 2005) can achieve the incorporation of damage parameters into constitutive models.

Finally, micromechanical models can describe the creep behavior of rock specimen when subcritical crack growth is the considered creep mechanism. There are micromechanical models describing the relation between micro-crack propagation and macroscopic rock behavior (Kemeny and Cook 1987; Ashby and Sammis 1990; Lockner 1993; Okui et al. 1993). As studies have verified the time-dependent microfracturing theory through creep tests on brittle rocks, the subcritical crack model can represent the timedependent behaviors such as creep and strain-rate related phenomena when incorporated into the micromechanical models (Sano et al. 1981). Kemeny (1991) extended the previous sliding crack model 
(Kemeny and Cook 1987) by combining it with the empirical Charles power law to describe the creep behavior. Lockner (1993) proposed a micromechanics model to describe the primary and secondary creep in which the cracks combined subcritical crack growth and the wing crack model. Okui and Horii (1997) used interaction field theory (Okui et al. 1993), a micromechanics-based continuum theory, to study the stress and time-dependent failure of brittle rocks. Brantut et al. (2012) proposed a model to describe time dependent brittle creep of water-saturated rocks under triaxial stress conditions based on the sliding wing crack model of Ashby and Sammis (1990).

In reviewing this literature, it is noticeable that studies have conducted many creep tests on different kinds of rock and have proposed various creep models to describe the creep behaviors. However, there is limited laboratory test data for coal measure rocks. In addition, because they proposed the creep models to describe the creep behavior specifically, they cannot extend them to represent the time-dependent behaviors of rocks in the post-failure region.

Although the creep of intact rock is an important source of roof convergence in underground coalmines, the measured roof convergence data is not only the manifestation of the time-dependent deformation of intact rock. Chin and Rogers (1987) recorded the diameter change of vertical shaft with $137 \mathrm{~cm}$ diameter at successive time intervals under constant radial pressure. They found that the viscosities determined in-situ are much lower than those derived from small intact specimens by up to several orders of magnitude. This means that the measured creep deformation is much larger than the creep deformation of intact rock specimens; as such, there are many factors contributing to roof convergence besides creep of intact rock.

Malan (1998) also observed this phenomenon in deep level hard rock mining. Underground closure measurement and observations demonstrate noticeable time-dependent behavior. However, the viscosity values determined from laboratory tests are two orders smaller for rock on a mining scale. This led to the belief that the rheological properties of the fracture zone, especially time-dependent formation fracture formation, must play a prominent role in the underground time-dependent behavior. He employed boundary element code, where the intact rock was simulated as viscoelastic Burgers material and the fractures were visco-plastic discontinuities, to simulate the underground time-dependent behavior. The model was randomly meshed with potential fractures considered to be intact with a prescribed strength. Once the potential fractures met failure criterion, they became real fractures with visco-plastic properties. The results show that the fracture zone relaxes in a time-dependent fashion transferring stress to the more solid rock, where the new fractures will form as a time-dependent process. From this study, it is observable that aside from the creep of intact rock, the property of failed rock and time-dependent failure are both important factors in time-dependent deformation.

Also, the time-dependent roof convergence can be the result of the behavior of bedding plane. The existence of bedding planes makes the roof as bonded rock layers. Separation between these layers can easily occur at the center of the openings. As a result, the measured convergence cannot represent the behavior of intact rock. However, when shear failure occurs at one opening corner, the rock layer will change from clamped beam into cantilever beam (Molinda and Mark 2010). This in turn significantly increases the whole roof deformation. 
In summary, the study of the time-dependent deformation and failure cannot consist merely of intact rock creep. The time-dependent failure, property of failed rock, and influence of bedding planes play important roles in the time-dependent deformation and failure of roof in underground coalmines. As this section conducted a literature review for creep of intact rock, the following sections will contain the literature review of these other aspects.

\subsection{TIME-DEPENDENT FAILURE}

\subsubsection{Laboratory study on the time-dependent failure of rock}

The formation of roof falls always involves rock failure. A proper failure criterion is critical for obtaining realistic results, especially when the phenomenon is time-dependent. As indicated by Sun and $\mathrm{Hu}$ (1997), a theory of strength of rocks should comprise two aspects: the condition of stress and the condition of time for rock failure, which can answer whether rock material fails under certain load and when failure takes place. However, the widely-used failure criterion (such as Mohr-Coulomb criterion, Drucker-Prager criterion, and Hoek-Brown criterion) can only answer whether rock fails under certain load, but not when failure takes place. Under creep condition, even when the applied load is lower than the failure load, rock failure may still occur after some time with creep deformation. The failure criterion based only on stress condition cannot account for this failure condition. Therefore, the time-dependent study should include one failure criterion accounting for the influence of time on strength.

Wawersik (1972) studied time-dependent strength of Westerly granite, Nugget sandstone, and Tennessee marble with uniaxial compression test with various strain rates and uniaxial creep test. The tests led to the conclusion that rock strength reduces with time. The observed decrease of the compressive strength of Tennessee marble with an increase of loading time was less than $15 \%$; conversely, the observed strengths of Westerly granite and Nugget sandstone dropped to $68 \%$ and $57 \%$, respectively. These results suggest that the long-term strength of Class II rock may lie considerably below their quasi-static compressive strengths; also, for Class II rocks, the long-term strength appears to be close to the quasi-static compressive strength. In addition, the study proposed one method to predict the failure of creep rock based on the complete quasistatic stress-strain curve. The maximum creep strain prior to failure is equal to the strain difference between two points at the same stress level in the ascending and descending parts of the complete stress-strain curve. If the creep strain can be theoretically calculated, researchers can in turn predicate the time to failure from the complete stress-strain curve.

When discussing the question of when failure can take place, the widely-used concept of time to failure can describe the time it takes for rock to fail under a constant load. Cruden (1974) presented a static fatigue law, $n \log \left(\sigma_{1} / \sigma_{2}\right)=\log \left(t_{2} / t_{1}\right)$, based on the hypothesis that the failure of rock happens when a critical density of microcracks were present, as well as a power law for transient creep. This method, based on the time to failure under a specific stress, can predict the time to failure of the rock under another stress value.

Schmidtke (Schmidtke 1985) tested 140 specimens of Lac du Bonnet granite to study the effect of longterm loading. The results show that there is an exponential relation between natural logarithm of stress level and time to failure. For a significantly long time, this relation can give a lower bound-static fatigue limit. The examination of the long-term test results determined a static fatigue limit of $45 \%$ of strength for the 
granite.

When analyzing the experimental results of tri-axial compression test and multi-stage creep test, Yamaguchi et al. (2001) introduced the concepts of representative strength and normalized failure time. They employed the assumption that rock deterioration rate is proportional to a certain function of stress, which in turn is logarithmic to the ratio of applied differential axial stress to the representative strength. Failure occurs when the integration of this stress function reaches some inherent value. Once the inherent normalized failure time can be obtained, one can determine the time to failure under other different test conditions, regardless of the stress path.

In addition, the concept of time to failure is useful specifically for creep condition; however, the strainrate test can also demonstrate the influence of time on strength. This is because each test duration will vary with different strain rates and as a result, this will involve different time. The resulting variation in strength can be the result of the influence of time. Shin et al. (2005) confirmed this by investigating the variation in strength and creep life of various sedimentary and igneous rocks from Japan with the concepts of stress dependence of creep life and rate dependency of strength. The study found that the stress dependency of creep life can quantitatively explain the rate dependency of strength, revealing that rock failure mechanism may be basically the same in both creep test and strength tests. This indicates that the parameters determined from either creep test or compression test with various strain rates can predict the behaviors of the other.

Bieniawski (1970) performed compression test with strain rates ranging from $10^{-6}$ to $10^{-9} / \mathrm{sec}$. The results show that a higher strain rate results in a higher elastic modulus, thereby resulting in a higher strength. Additional researchers observed the phenomenon of reducing rock strength with decrease in strain rate during compression tests as well (Hudson and Brown 1972; Peng 1973; Sano et al. 1981).

\subsubsection{Incorporation of time-dependent failure into constitutive model}

Failure criterion has incorporated the influence of time on strength to account for the time-dependent failure. The review of this literature illustrates that the time-to-failure for creep condition can determine the influence of time, as well as using the relationship between strength and strain rate for compression test. However, these concepts cannot be directly included into failure criterion. Failure criterion usually incorporates the time-dependent failure by means of time-dependent strength or strength deterioration.

On one hand, time-dependent strength can represent the influence of time, which indicates that the strength will decrease with time. Kaiser $(1980,1981)$ proposed a phenomenological model for rock with time-dependent strength. This time-dependent strength works for peak strength and for post-peak strength. Malan (1999) also derived a time-dependent Mohr-Coulomb failure criterion, where the cohesion degraded with time and the degradation rate depended on the distance between the stress state and the residual strength. This model was only effective for post-peak strength, and time did not affect the peak strength.

On the other hand, strength deterioration can also describe the time-dependent failure. As stated by Drescher and Handley (2003), creep itself is not a driving mechanism of failure; it is merely an outwardly measurable manifestation of failure processes taking place in a stressed material. This means that the timedependent deformation can be considered as a damage factor and, as a result, the strength will degrade with 
time-dependent deformation. Therefore, the failure criterion can directly incorporate the influence of time.

Lau and Chandler (2004) used damage-controlled test and long-term loading test to study the damage evolution with time and its influence on material properties. The study on time to failure under constant load shows that a shorter time is necessary to fail the specimen if the stress ratio is higher. The stress value when unstable crack growth begins would be a conservative estimate of long-term strength at any confining pressure, particularly at low confinement. In addition, they quantified the damage as a function of time by determining the degradation of elastic properties through periodic unloading-reloading cycles. As damage increases, Young's modulus decreases, and Poisson's ratio increases. The study of Lin et al. (2004, 2009) shows similar results, where the properties were determined by the change of compressive wave and shear wave velocities. This study quantified the damage using the following factors: inelastic volumetric strain, degradation of dynamic elastic properties, and accumulative acoustic emission count during the uniaxial constant loading test on granite specimen.

In the viscoelastic-plastic model Fakhimi and Fairhurst (1994) proposed to investigate the stand-up time of underground excavations, strength degrades exponentially with accumulated viscous deformation. The influence of accumulated viscous deformation can affect the peak strength and the strain-softening behavior as well.

Ray (2009) also studied the time effects on cutters and roof falls in underground coalmines. Because there is not enough rock test data available, the study employed strength reduction method was employed to account for the time-dependent strength degradation. There is no cutter observed with in-situ peak strength, but it gradually begins to develop as the peak strength is reduced.

Sterpi and Gioda (2009) reduced the viscoplastic creep parameters with accumulated viscoplastic strains to account for the gradual mechanical damage. As a result, the strain will gradually accelerate and transit from secondary stage into tertiary stage. Yang et al. (2014) and Liu et al. (2015) also used similar rheological models to represent the creep behavior of diabase and rock salt, respectively.

Based on their micromechanical model, Brantut et al. (2012) proposed an approximate creep law, where the subcritical crack propagation reduces the failure stress in a manner equivalent to a decrease in cohesion.

Because the time-dependent behavior in post-failure region is part of this study, a time-dependent strength law or strength deterioration law is preferable. The time-dependent strength relates strength to time directly; the strength deterioration law usually relates strength to viscous deformation, which in turn is related to time and stress level.

\subsection{BEHAVIOR OF FAILED ROCK}

The literature review for the behavior of failed rock is divided into two parts. The first part is focused on strain-softening behavior, usually considered to be a time-independent behavior. It describes the use of strain-softening model to simulate cutter roof failure in underground coalmines (Gadde and Peng 2005; Ray 2009; Sherizadeh and Kulatilake 2016) and demonstrates that the strain-softening model is capable of capturing the progressive formation of cutter type roof failure. The second part contains the review of the time-dependent post-failure behaviors of rock. 


\subsubsection{Strain-softening behavior}

Strain-softening behavior is an important characteristic of failed rock. Although strain-softening behavior is the result of inhomogeneous deformation and fracturing (Read 1984; Kimura et al. 1987), rather than a true material property of rock, studies still widely use the strain-softening model to investigate the stability of underground excavations and slopes.

To analyze the effects of the post-failure stress-strain behavior on tunnel support, Azzouz and Germaine (1981) presented a closed-form solution for a cylindrical opening with Tresca yield criterion and no gravity; they divided the surrounding rock mass into three zones (plastic zone, softening zone, and elastic zone), based on the idealized stress-strain curve. The results show that the size of the yield zone decreases as the strain required from peak stress to residual stress increases, as well as when the stress drop from peak stress to residual stress decreases. While the influence of dilation in post-failure region on the size of yield zone varies for different normalized load factors, which is the ratio between stress difference of far-field in situ stress and the internal opening pressure, and peak-strength of the surrounding rock mass. Dilation increases the size of yield zone with smaller degrees of unloading while decreasing the size of yield zone with higher values of load factors.

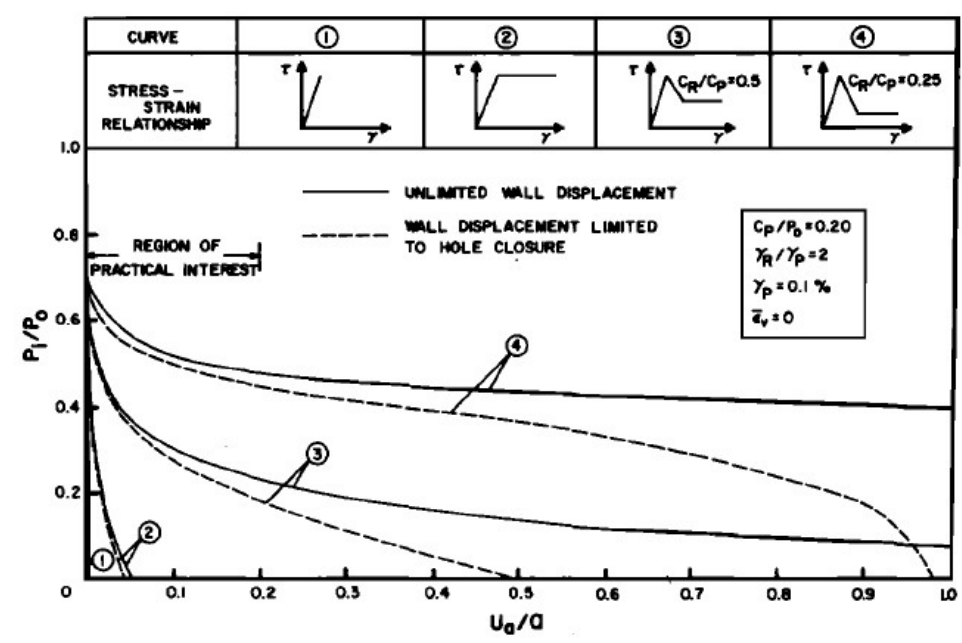

Fig. 2.18. Ground reaction curves for materials with different stress-strain relationships (Azzouz and Germaine 1981)

Sterpi (1999) studied the strain localization and the associated softening effects with a finite element model. The numerical results show that strain-softening behavior has significant influence on the overall behavior of the surrounding material, while one with elastic-perfectly plastic model cannot present. The results also indicate that only the softening analysis provides some information about the development of collapse mechanism, and its shape is in qualitative agreement with the experimental observation of the three-dimensional model.

After realizing that elastic model, elastic-perfectly plastic model, and elastic-brittle model cannot predict the extent and depth of failure in a circular test tunnel in brittle rock, Hajiabdolmajid et al. (2002) proposed 
a model where the cohesion weakens and frictional strengthens (CWFS) as function of plastic strain. The model successfully captured the essential component of brittle rock mass failure.

Fang and Harrison $(2001,2002)$ proposed an elemental degradation method to simulate the progressive failure of rock where the degradation index represented the elemental degradation. This index is the ratio of the degradation occurring at a certain confining stress level to the corresponding degradation occurring under uniaxial conditions. They combined this theory with an elasto-plastic model to obtain an elasticbrittle-plastic model and incorporated it into FLAC to simulate the progressive failure of coal pillars.

Gadde and Peng (2005) proposed the use of the strain-softening model with implementation of cutting sequence to capture spatial distribution of cutters close to field observation at an Illinois mine. Ray (2009) further adopted this method for the purpose of checking the influence of cutting sequence on cutter formation. This captured the failure initiation and propagation within roof by the distribution of cohesion value during progressive mining development. Studies have also used strain-softening model in the simulation of bedded mine roofs (Esterhuizen and Bajpayee 2012; Sherizadeh and Kulatilake 2016).

In summary, the literatures reviewed here demonstrate the importance of incorporating strain-softening model in capturing the realistic and/or progressive failure in numerical simulation. Also, the distribution of shear plastic strain or cohesion value can demonstrate the failure condition.

\subsubsection{Time-dependent post-failure behavior}

Aside from strain-softening behavior, which is time-independent, failed rock can show time-dependent post-failure behavior, differing from that in the pre-failure region. However, it is difficult to maintain a constant load on a failed specimen. There is only one group of creep tests reported to be conducted on marble and sandstone in the post-failure region (Peng 1973). The results reveal that the failed rock can sustain creep load but the time to creep rupture is usually within 10 minutes. If the failed rock has not been fully separated by fractures into pieces, the creep curves usually consist of secondary and tertiary stages while the creep curves for the fully separated specimens holding by friction, consist of three stages.

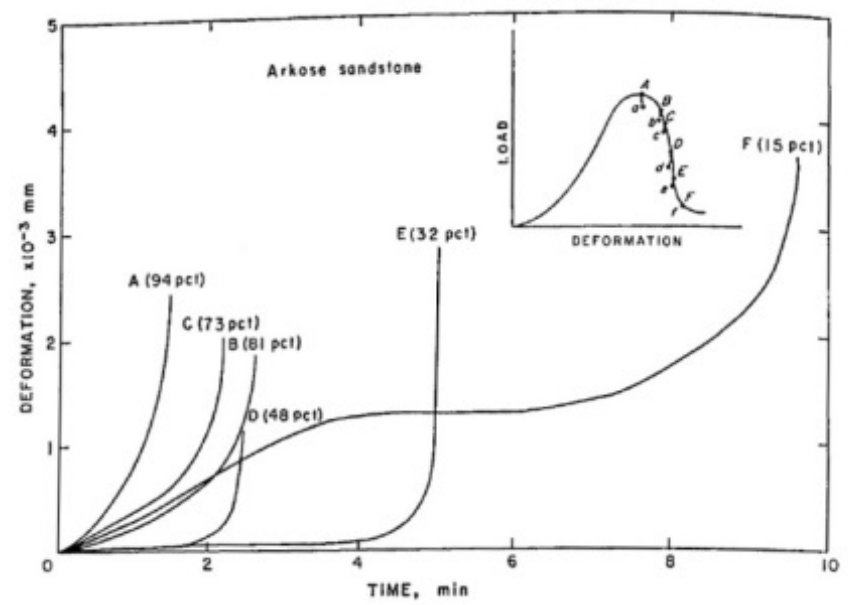

Fig. 2.19. Creep curves of fractured Arkose sandstone (Peng 1973) 
Instead, most studies used relaxation test and strain-rate test to investigate the time-dependent postfailure behavior of rock. During relaxation test, the displacement is a fixed value and the researchers can monitor the relation between stress and time; the strain-rate tests are compression tests with different strain rates. Using different strain rates, compression tests will involve different time, resulting in differences in the stress-strain curve.

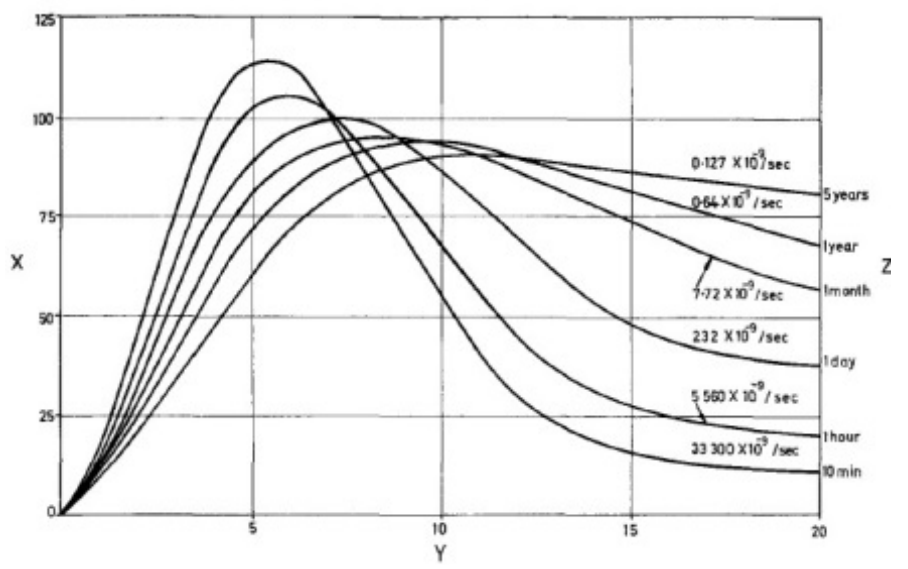

Fig. 2.20. Stress-strain curves with different strain rates (Bieniawski 1970)

Bieniawski (1970) first investigated the time-dependent behavior of failed rock, conducting three different tests on find-grained sandstone: one with constant strain rate, one with changing strain rate, and one with constant load. The tests with constant strain rate showed that a higher strain rate resulted in a higher elastic modulus and in a higher strength and that lower strain rate led to flatter slope of loaddeformation curve in post-failure region; Hudson and Brown (1972) confirmed this in their research on marble. A sudden change strain rate in post-failure region produced a sudden and corresponding reaction. For a sudden decrease of strain rate, the load-deformation curve became steeper, and then became flatter, similar to that of the low strain rate. While suddenly increasing the strain-rate resulted in increased strength and then steeper load-deformation curves as that of high strain rate. The tests with constant load revealed that failed rock could eventually reached stability under constant applied load, but the previous straining history affected this process.

Instead of creep tests, Hudson and Brown (1972) conducted relaxation tests with different constant strain rates $\left(10^{-4}\right.$ and $\left.10^{-6} / \mathrm{sec}\right)$ on marble to study the time-dependent behavior of rock within post-failure region. The results showed that the relaxation effect is greater for a faster displacement rate, which they then used to predict the effect of displacement rate on the shape of the complete force-displacement curve by integrating the relaxation during each displacement increment along the displacement axis at the different rates. The pre-failure part of the predicted force-displacement curves and the strength follow the relaxation trend exactly; however, the post-failure part of the predicted curves was the reverse of the experimental results. 


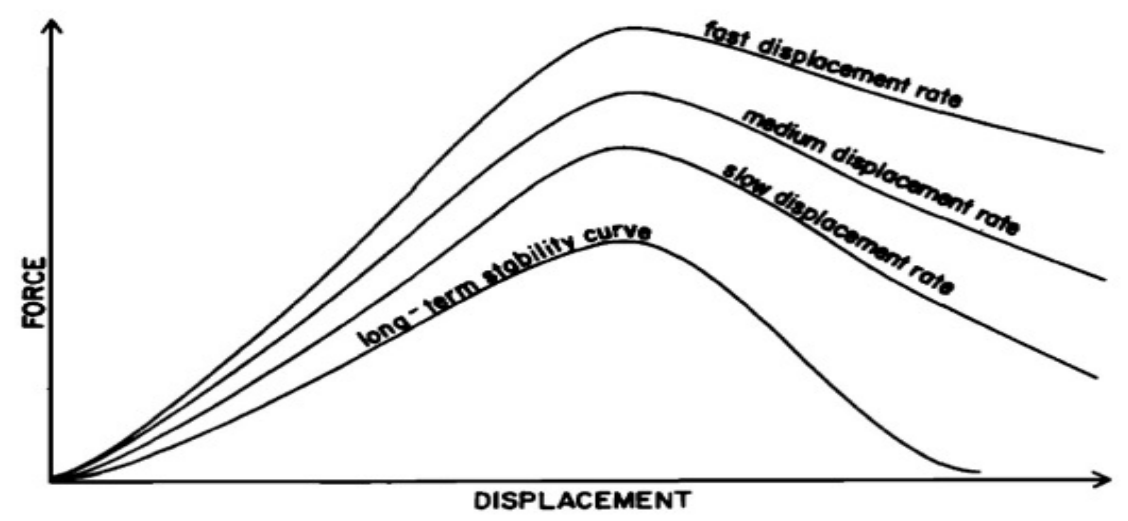

Fig. 2.21. Hypothetical variation in complete force-displacement curves due to relaxation alone (Hudson and Brown 1972)

Peng and Podnieks (1972) conducted relaxation and strain-rate tests on different rocks to study timedependent behavior. The tests on tuff with strain rates ranging from $10^{-2}$ to $10^{-7} / \mathrm{sec}$ showed that the strength increased with increasing strain rates, and the difference of stress-strain curves for different strain rates occurred after reaching the elastic limit. They also applied varied strain rates on each specimen after elastic limit and before failure, revealing that the crack propagation caused the stress relaxation. It also revealed that if the applied strain rate is high enough to make the increase of the applied stress greater than the stress relaxation, the applied stress continues increasing. Otherwise, the applied stress will drop.

Aside from laboratory tests, studies have proposed a few models to describe the time-dependent postfailure behaviors. Based on the laboratory tests by Bieniawski (1970) and Peng (1973), Kaiser (1980, 1981) proposed a phenomenological model for rock with time-dependent strength, incorporating the ratedependent phenomenon, strain softening behavior, creep, and relaxation, to study the effect of stress history on the deformation behavior of underground openings. The results show that the load-history, particularly the time-dependent post-peak strength behavior of rock, is important in evaluating the stability of underground openings because the extent and shape of the plastic zone and the tunnel wall displacements can vary within a wide range depending on the rate of excavation, the excavation layout, and the type of support.

Fakhimi and Fairhurst (1991) also developed a viscoelastic-plastic model for intact rock to predict the stand-up time of underground excavations. Per this study, the rock will show softening behavior with plastic strain and viscous strain to account for the degradation of rock strength. The simulation of plane strain problems with this model shows qualitative good agreement with laboratory results, such as rate-sensitivity, creep dilatancy, and time-to-failure.

Nawrocki and Mroz (1998) proposed a visco-plastic model for rocks in which both yield and viscosity undergo variation during the deformation process. They used a Bingham-type model composed of elastic and viscous portions with its behavior governed by the elastic modulus, the yield stress, the residual stress, and viscosity coefficient. The modulus remains unchanged, whereas they proposed that the viscosity coefficient would reduce nonlinearly from initial value to residual value along with strain. In addition, yield 
stress decreases following the degradation rule. The degradation of yield stress consists of two parts: deformation degradation and stress degradation. The viscous strain induced the deformation degradation, while the stress degradation represents the crack growth due to stress increase. With such a model, they can meaningfully describe the rate-dependent strength and time-dependent strain-softening behaviors.

When investigating the time-dependent closure behavior of tabular excavation in deep gold mines, Malan (1999) proposed an elasto-visco-plastic model. The model used a time-dependent Mohr-Coulomb yield surface and time-dependent cohesion to account for strength degradation with time. This model was then employed to simulate various time-dependent laboratory tests, including compression test with various strain rates, and relaxation test in post-failure region (Malan and Drescher 2000). The result shows that the model can be only partially successful in simulating the laboratory behavior. The model can capture the increase in strength with increasing strain rates, different failure modes with varying strain rates, and the decreasing axial stress during relaxation. However, there are two points contrary to the laboratory tests. The first one is that the model predicts a decreasing negative slope in the post-failure region with higher strain rate. The second one is that, for relaxation tests initiated at different stress levels, the eventual residual stress values are identical.

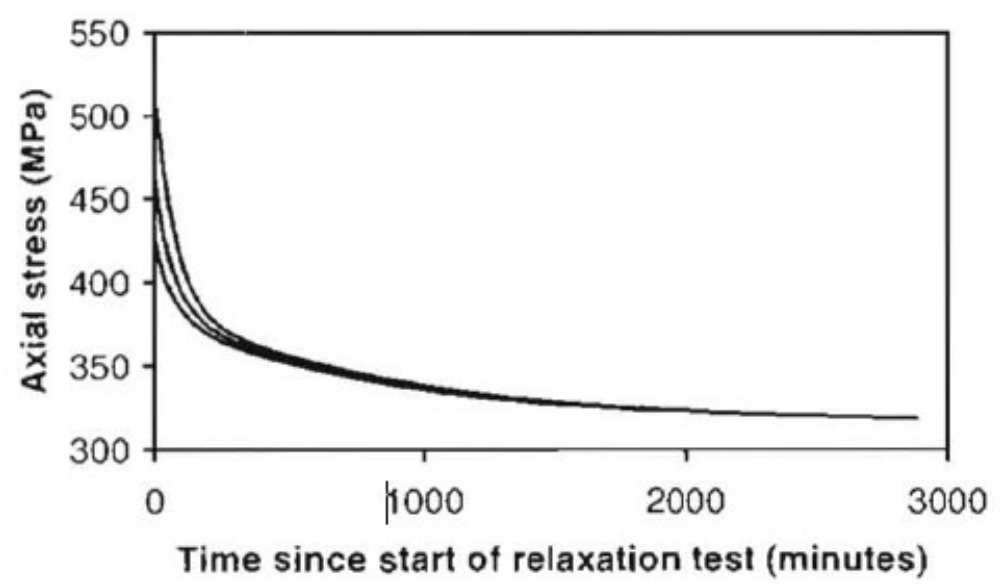

Fig. 2.22. Relaxation behavior for the three tests initiated in post-failure region (Malan and Drescher 2000)

In summary, the time-dependent post-failure behavior of rock is an important feature of failed rock and must be incorporated into a constitutive model to investigate the influence on the time-dependent stability of underground mine roof. The literature reviewed here demonstrates that the time-dependent behavior can still occur during the strain-softening process, and there is significant difference in the time-dependent properties between intact and failed rock. Therefore, it is necessary to conduct laboratory tests to investigate the time-dependent behavior of failed rock and incorporate the time-dependent post-failure behavior into a constitutive model to study the influence on the time-dependent response of roof stability.

\subsection{BEDDED ROCK}

Another common problem in underground coalmines is bedded roof. The bedding planes can decrease the rock mass strength and increase the failure area. Bedding planes also introduce anisotropy, affecting the roof failure mode. Time-dependent deformation can also occur along these discontinuities. 


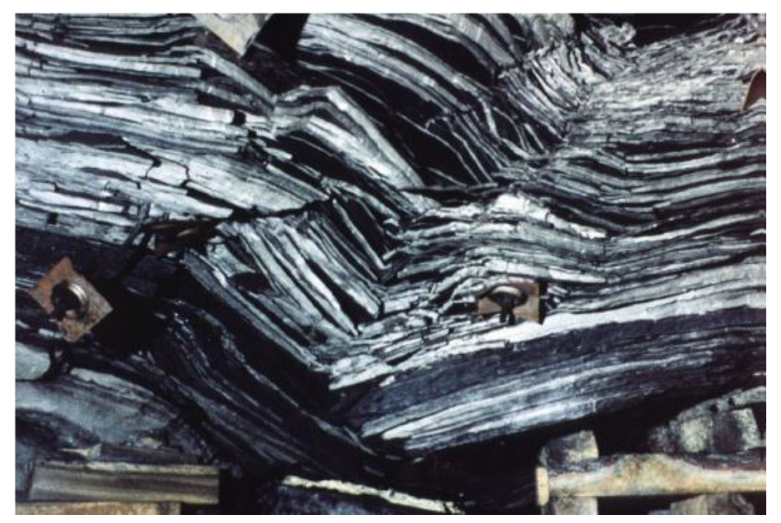

Fig. 2.23. Cutter failure in bedded roof (Esterhuizen and Bajpayee 2012)

\subsubsection{Time-independent influence of bedding planes}

The time-independent influence of bedding plane on rock mass can be shown in one of two ways. On one hand, the existence of bedding planes can decrease the rock strength and in turn induce more failure in rock mass. On the other hand, the presence of bedding planes induces anisotropy into rock mass and therefore alters the stress distribution and the failure mode.

(1) Influence of bedding planes on strength

The triaxial compressive test conducted on three bedded rocks and one isotropic rock showed that bedded formations exhibited sizable direction variations in both their elastic constant and yield strengths (Chenevert and Gatlin 1965). The bedding planes induced an appreciable difference between properties in the horizontal and vertical directions. Strength reduction was as high as 40 percent with the test specimens orientated at $20-30^{\circ}$ to the bedding planes.

Einstein and Hirschfeld (1973) systematically investigate the influences of a joint system on the deformability and strength of a rock mass of gypsum material. The results show that the strength of a jointed rock is at minimum if the joints are favorably inclined, and the strength increases if the joints are unfavorably inclined. This is because the failure gradually changes from sliding along the pre-existing joints to fracturing through the intact rock material. Aside from this, a generalized Mohr plot was presented to represent the behavior of the model rock mass with various joint configurations. The upper limit of the strength of the jointed rock is the Mohr envelope of intact material and the lower limit is the Mohr envelope for sliding along a single smooth joint. 


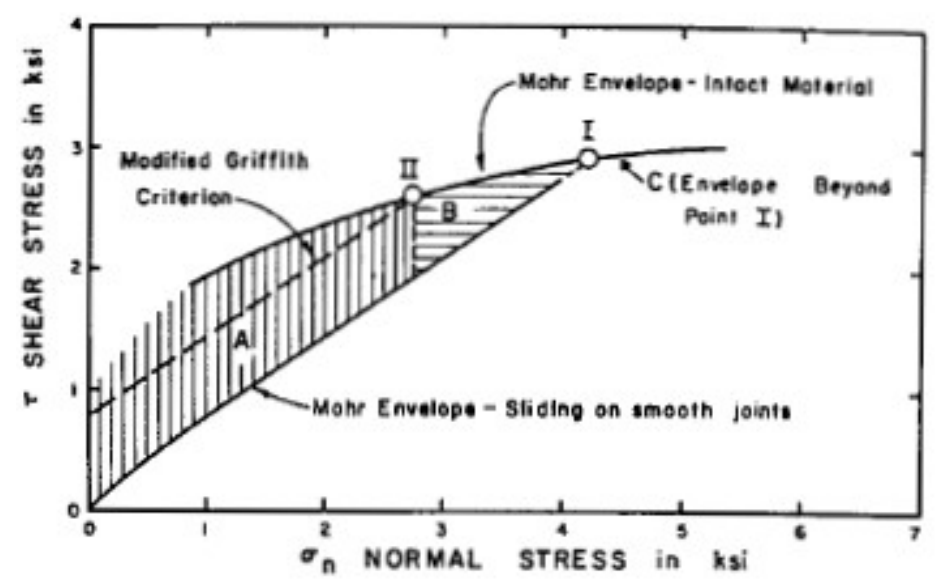

Fig. 2.24. Mohr envelope for different joint configurations (Einstein and Hirschfeld, 1973)

Kulatilake (1985) numerically investigated the elastic constants and strength of rock with discontinuities. The simulation method consisted of probabilistic modeling of discontinuity geometry, generation of discontinuities in rock blocks by Monte-Carlo simulation, and finite element analysis of simulated rock blocks. The results demonstrate that the mean value of the ratio between shale mass strength and intact shale strength was about 0.6 ; for modulus, the ratio was about 0.45 .

Ramamurthy and Arora (1994) conducted 250 uniaxial compression tests and 1300 triaxial tests on jointed and intact specimens of three materials: plaster of Paris, Jamrani sandstone, and Agra sandstone. A joint factor was proposed to account for the influence of the number of joints per meter length, joint orientation, and the shear strength along this joint on strength. This factor is uniquely related to the ratio of compression strength of jointed rock to that of the intact rock, irrespective of the rock type.

Schultz (1995) conducted a literature review about strength and deformability of jointed basaltic rock mass. The review documented marked reductions in strength. Tensile strength and cohesion of the rock mass are approximately one to two orders of magnitude lower than the corresponding values for intact basalt. Uniaxial compressive strength is lower by approximately an order of magnitude; the deformation modulus is smaller by perhaps a factor of two, relative to values of Young's modulus for intact basalt under condition of ambient temperature and negligibly small confining pressure.

Gadde et al. (2007) proposed a simple method to estimate the strength reduction factor (RF) based on the laboratory uniaxial compressive strength, the diameter or the edge length of the tested samples, $d$, and the thickness of the stratum between any two adjacent bedding planes, $T$.

$$
\begin{array}{ll}
R F=(T / d)^{-0.5} & \text { if } \mathrm{T}<48 \text { inches } \\
R F=(48 / d)^{-0.5} & \text { if } T \geq 48 \text { inches }
\end{array}
$$

In the study of horizontal stress effects on roof stability in US limestone mines, Esterhuizen et al. (2008) simulated the influence of bedding planes on failure. Without bedding plane, failure can extend up to abut $3 \mathrm{~m}$ above the roofline. With only one $1 \mathrm{~m}$-thick bedding plane in the roof, failure extends to $4 \mathrm{~m}$ above the roofline. Failure can also extend to up to $5 \mathrm{~m}$ when including three $1 \mathrm{~m}$-tick bedding planes into the roof; 
however, when modelling the roof as a thinly bedded rock with ubiquitous joint model, the extent of failure is much greater to about $10 \mathrm{~m}$ above the roof line. Inspection of the results demonstrates that slip along the roof beds allows more roof deflection to occur and therefore reduces the confinement in the roof.

(2) Influence of bedding plane on failure mode

Stephansson (1971) derived the mathematical solutions of deflection, bending moment, and longitudinal fiber stress of seven different configurations of single- and multi-layer roof in horizontally bedded rock and verified these solutions with physical models. The results demonstrate that the abutment compression has a great influence upon stress and deflection around underground openings. In the model experiments, thin single- and double-layer roofs affected by a uniform loading on the top surface show a beam-like breakage pattern, with the first failure beginning in the roof center followed by failure at a certain distance from the wall. Thick roof layers obtained $\mathrm{H} / \mathrm{l}>0.5$ arching failure mode. The failure starts at the bottom part of the center line and moves upward, leading to high shear stresses at the walls of the openings. This increasing shear stress finally results in a momentary failure process from the bottom of the roof and proceeds towards the top of the existing crack at the center line.

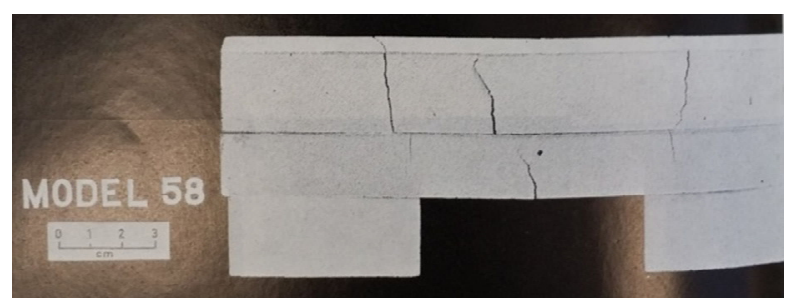

Fig. 2.25. Breakage patterns of centrifuged models with layer thickness less than half the roof span (Stephansson 1971)

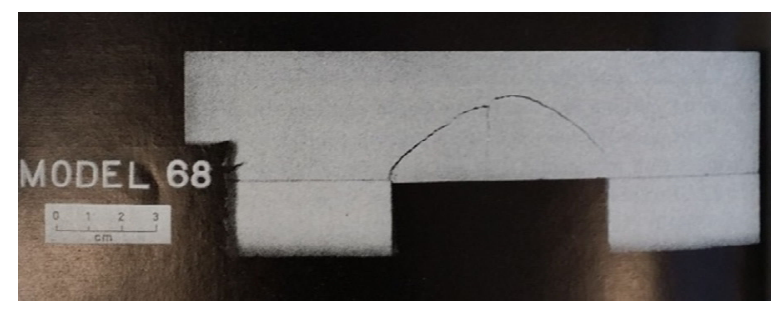

Fig. 2.26. Breakage pattern of centrifuged models with a layer thickness greater than half the roof span, $\mathrm{H} / \mathrm{l}=0.57$ (Stephansson 1971)

Stefanizzi et al. (2007) used the extension strain criterion (Ndlovu and Stacey 2007) with ELFEN code to investigate the strain driven fractures around tunnels in layered rock masses. The results showed that either the presence of discontinuities or the change in lithology affected the mechanism of failure around the tunnel.

When studying the influence of cutting sequence on cutters and roof falls, Ray (2009) also investigated the influence of bedding plane on the severity of the cutter problem. The study observed that the discontinuities did not significantly change the cutter patterns noticed near the roof surface. However, the depth to which cutters propagated in the roof depends on the number and position of the bedding planes. The presence of bedding planes breaks the roof's continuity and as a result, the discontinuities can arrest 
the cutter propagation deep into the roof. With increasing plastic strain in the roof, the cutters propagated horizontally as the failed roof elements shed their load to the adjacent stronger elements.

Perras (2009) studied the influence of lamination thickness on anisotropic behavior at Niagara Tunnel, Canada, by modeling the laminations ranging between 0.16 to $16 \mathrm{~m}$ in thickness. The study found an existing a critical normalized lamination thickness (thickness/radius) of 0.9 , above which the excavation response is similar to the equivalent isotropic model and below which the laminated behavior corresponds to a characteristic failure mode controlled by bed deflections and bed parallel shear. As the normalized lamination thickness initially decreases below 0.9, the stresses are channeled through the crown beam, concentrating the yield area and increasing the crown deflections; this leads to crown beam failure. As the lamination thickness further decreases, the stresses are shed to multiple laminations, increasing the displacements significantly and changing the shape and extent of the yield zone. From multiple lamination coupling to self-limiting yield, the degree of tensile yielding controls the development of chimney style failure. Tensile yielding first begins in the haunch area and progressively extends above the crown, as the lamination thickness decreases; this continues until it reaches a self-limiting plastic yield zone shape at normalized lamination thickness below 0.026. Incorporation of discrete anisotropy is necessary to accurately model the excavation response in horizontally lamination ground.

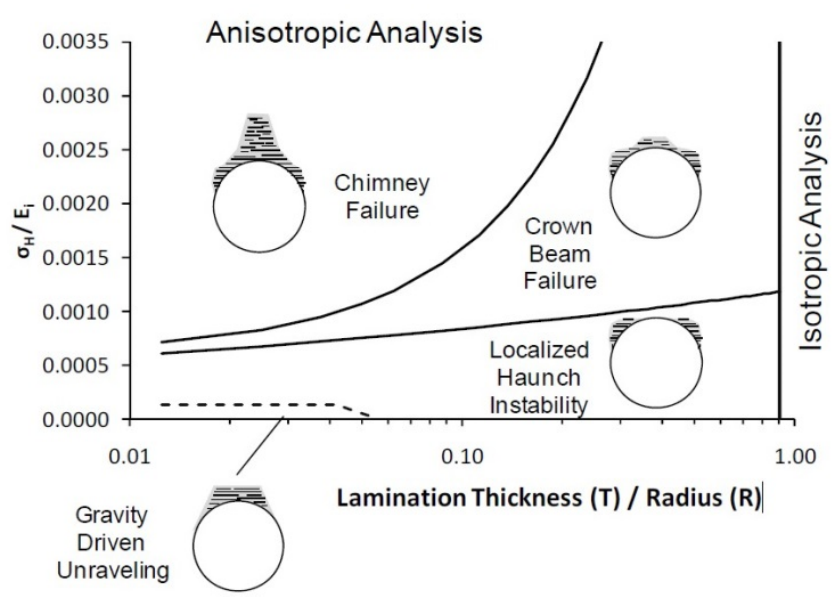

Fig. 2.27. Normalized horizontally laminated anisotropic ground behavior chart showing when anisotropic plasticity analysis should be conducted and generally what type of anisotropic behavior can be expected (Perras, 2009)

The numerical simulation performed by Esterhuizen et al. (2012) shows that bedding planes in roof have a detrimental effect on the stability of the roof. Slip along the interfaces can cause an increase in the horizontal stress in the roof and a decrease in the confining stress. The net effect is that the laminated roof is more likely to fail than a massive roof of the same strength. Cutter type failure at the excavation corners occurs in the numerical models through a process of compressive shearing failure, followed by downward rotation and tensile fracturing of the immediately adjacent rock. However, failure at the center of the roof span occurred more frequently when the rock strength was relatively higher, and the interface friction was lower than the base case analyses.

In summary, the presence of bedding planes significantly changes the behavior of roof in underground coalmines. Due to sliding along bedding planes or change in stress distribution, the bedding planes can 
decrease the strength of rock and weaken the roof. This induces more failure in roof and therefore increases roof instability. However, the magnitude of strength decrease depends on various factors, such as orientation, thickness, and joint property. In addition, the existence of bedding planes alters the failure mode. The bedding planes separate the roof into thin layers or beams, which are easy to separate and susceptible to bending or buckling. The failure of one layer decreases the confinement to the inner layer and further induces progressive failure, layer by layer. The initiation and development of this failure process also depend upon many factors, including thickness, joint property, in-situ stress, and mine layout. Finally, these studies demonstrate that the influence of bedding plane on roof behavior is complicated. Simulating bedded roof merely with anisotropic model or by decreasing rock mass strength cannot lead to realistic results. Therefore, it is necessary to simulate the bedded roof by explicitly incorporating the joint model into roof during simulation.

\subsubsection{Time-dependent influence of bedding planes}

Aside from the influence on time-independent behaviors of rock mass, bedding planes can also exhibit time-dependent behaviors, which may in turn affect the overall behavior of rock mass. As pointed out by Bowden and Curran (1984), the concern for the creep behavior of joints was due to their pervasiveness around underground excavations, as well as the fact that the shear strength of a joint was generally less than that of intact rock. The following summarizes the important conclusions of previous laboratory tests.

The creep behavior of rock joints, in general, was similar to that of an intact rock, although creep deformations were usually greater for the former (Amadei 1979). For the rock with joints, the creep strain was higher than that for the equivalent intact specimen, particularly in the early stages and increasing inclination from $30^{\circ}$ to $60^{\circ}$. Decreasing the cohesion to zero also led to higher creep (Schwartz and Kolluru 1981). For given surface properties, creep displacement along discontinuity depended on the ratio of the applied shear stress to the peak shear strength of the joint, meaning that above a certain value of this ratio, joint creep became the main component of movement; below this value, creep of the intact rock was dominant (Amadei 1979; Schwartz and Kolluru 1982; Bowden and Curran 1984; Malan 1998). Additionally, joint creep also depends upon the absolute stress level across the joint (Schwartz and Kolluru 1982).

The time-dependent behavior of a jointed rock mass is a function of the viscous behavior of the intact rock and its discontinuities. However, the simulation results with displacement discontinuity method (where the intact rock was modelled as a linear viscoelastic material while the joints were modeled with a nonlinear time-dependent model) demonstrated that joint creep was only important when joint surfaces were relatively free to move. This is not the situation normally encountered in underground coal mines where the dominant discontinuity is the horizontal bedding planes. Additionally, Nadimi et al. $(2010,2011)$ obtained input parameters for 3DEC from laboratory and field data to simulate the time-dependent deformation of Siah Bisheh cavern, which only considered the influence of discontinuities and creep of rock. The simulation results show good agreement with field data. From this point of view, it is not necessary to consider the time-dependent behavior of discontinuities. 


\subsection{SUMMARY}

From the literatures discussed in the previous sections, it can be noted that all the reviewed topics are related to the research in this dissertation -the time-dependent deformation and associated failure of roof in underground mines. Below is a summary of multiple important conclusions from this literature review.

First of all, the laboratory tests on intact and failed rock specimens demonstrate that rock has a significant variation in the time-dependent property before and after the peak. It is necessary to determine the time-dependent property at different stages of a complete stress-strain curve. However, it is difficult to conduct a creep test on a failed specimen and as a result, there is limited knowledge about the timedependent behavior of failed rock. Rather than creep test, researchers have successfully performed relaxation test on failed specimens. The time-dependent behavior in the post-failure region was observable from the relaxation test. However, no studies attempted to determine the time-dependent properties or parameters from these relaxation tests. If relaxation test can determine the time-dependent parameters, relaxation test method can be the test method to determine the time-dependent behavior and associated parameters at different stages of a complete stress-strain curve.

Second, the influence of the variation in the time-dependent property and the influence of timedependent rock failure on roof stability should also be a significant part of this study. The literature demonstrates that, for the time-dependent study on roof stability, special attentions should be paid to the variation in the time-dependent property, strain-softening behavior, and the time-dependent failure of rock. Studies have widely used strain-softening model in the stability analysis of underground structures. The influence of the other two factors on roof stability should be the first research priority, ensuring the necessity of including all these features into a new constitutive model.

Third, this study will propose a constitutive model capable of describing the time-dependent behavior before and after failure, strain-softening behavior, and strength deterioration if their influence on the timedependent roof stability is confirmed. Previous studies proposed models that specifically represent the creep behavior. Even if the model can describe the three stages of a creep curve, it is still difficult to account for the strain-softening behavior. If laboratory tests can determine the time-dependent behavior in the prefailure and post-failure region, strength deterioration, and strain softening behavior, it will be possible to obtain such a model, which includes all the important characteristics, either time-dependent or timeindependent, of rock.

Fourth, various factors can affect the roof failure process, and their influence should be studied from a time-dependent point of view. Previous studies demonstrate that directional horizontal stress and mine layout are major factors that can affect mine roof stability. During development, the roof failure initiates due to these time-independent factors. The roof will then experience time-dependent deformation and failure propagation when the entry is in service. The proposed constitutive model will simulate the timedependent deformation and failure of roof in underground coalmines under different conditions. As a result, it can determine the influence of these factors on the time-dependent deformation and associated failure of mine roof. 
Finally, this study should investigate the influence of bedding plane and roof support on the timedependent deformation and failure of roof in underground coalmines. Previous studies demonstrate the importance of bedding planes in affecting rock mass behaviors. The presence of bedding planes in the mine roof decreases the strength of rock mass and induces more failure in the roof at equilibrium, which may affect the following time-dependent failure process. In addition, the bedding planes divides the roof into beams or layers, inducing progressive failure layer by layer. Under time-dependent conditions, the separated and bended beams can achieve more deformation and further decrease the confinement to the upper layer, inducing more failure. Therefore, the time-dependent simulation of mine roof should consider the influence of bedding planes. 


\section{CHAPTER 3 \\ STUDY OF THE TIME-DEPENDENT PROPERTIES OF ROCK WITH THE RELAXATION TEST}

\subsection{INTRODUCTION}

Measured or observed roof deformation over time is not merely the result of the creep of intact rock. The closure data for underground excavations demonstrates that the viscosity values determined from laboratory test on intact specimens are much larger than that on an engineering scale (Chin and Rogers 1987; Malan et al. 1997). Measurements indicate that, aside from the creep of intact rock, there are other factors contributing to the time-dependent deformation. Malan et al. (1997) studied time-dependent behavior for deep level stopes in gold mines with a boundary element code. The intact rock was simulated as Burgers material, and the fractures were treated as viscoplastic. A random mesh of potential fractures covered the problem space, and they were initially intact with a prescribed strength. Upon failure, the potential fractures became viscous discontinuities. The results indicate that the rheological behavior of the fracture zone and the resulting time-dependent formation of new fractures governs the time-dependent behavior. The fracture zone relaxes over time, transferring stress to the more solid rock. New fractures then form in these positions over time. The observed behavior demonstrates the importance of the timedependent behavior of failed rock and the time-dependent failure process in the time-dependent closure of underground excavations.

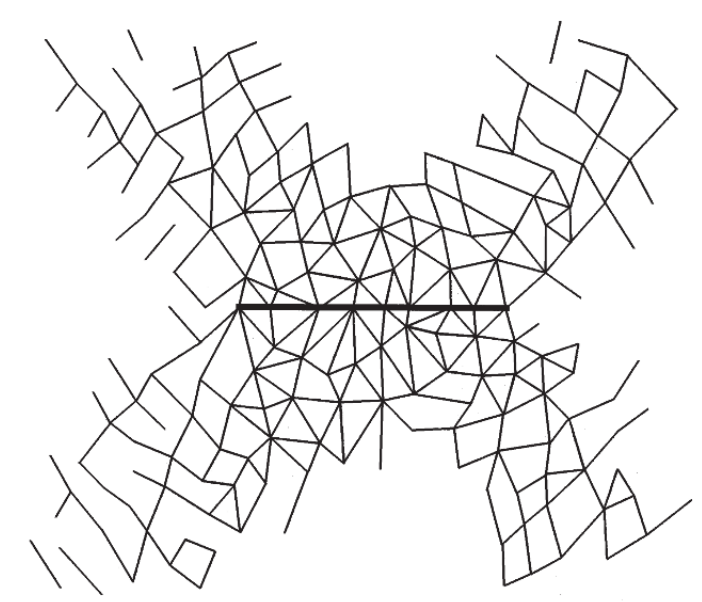

Fig. 3.1. Development of fracture zone 24 hours after the 9 th mining increment. The stope is represented by the thick black line (Malan et al. 1997)

Determination of rock time-dependent parameters for intact and failed rock is the key in numerically simulating the time-dependent failure process of underground mine roof. Creep test has been the primary test method conducted to study the time-dependent behavior of rocks (Price 1964). The relation between strain and time can be determined when a constant load is applied onto a rock specimen and the associated 
creep parameters can be determined by fitting the creep model to the creep curves. In the tertiary stage of a creep test, the time-dependent strain starts to accelerate and departs from the trend of the second stage. Extra parameters are usually necessary to represent the departed strain by fitting the creep curves of tertiary stage. However, before the final failure, the rock specimen is still intact in the tertiary stage. The determined parameters cannot represent the time-dependent property of failed rock. In addition, once the rock specimen fails, it is extremely difficult to maintain a constant load (Peng 1973). As a result, limited knowledge is available about the time-dependent property of rocks in the post-failure region; therefore, previous studies assume that creep parameters remain constant before and after failure (Fakhimi and Fairhurst 1994; Lorig and Calderon Rojo 2002).

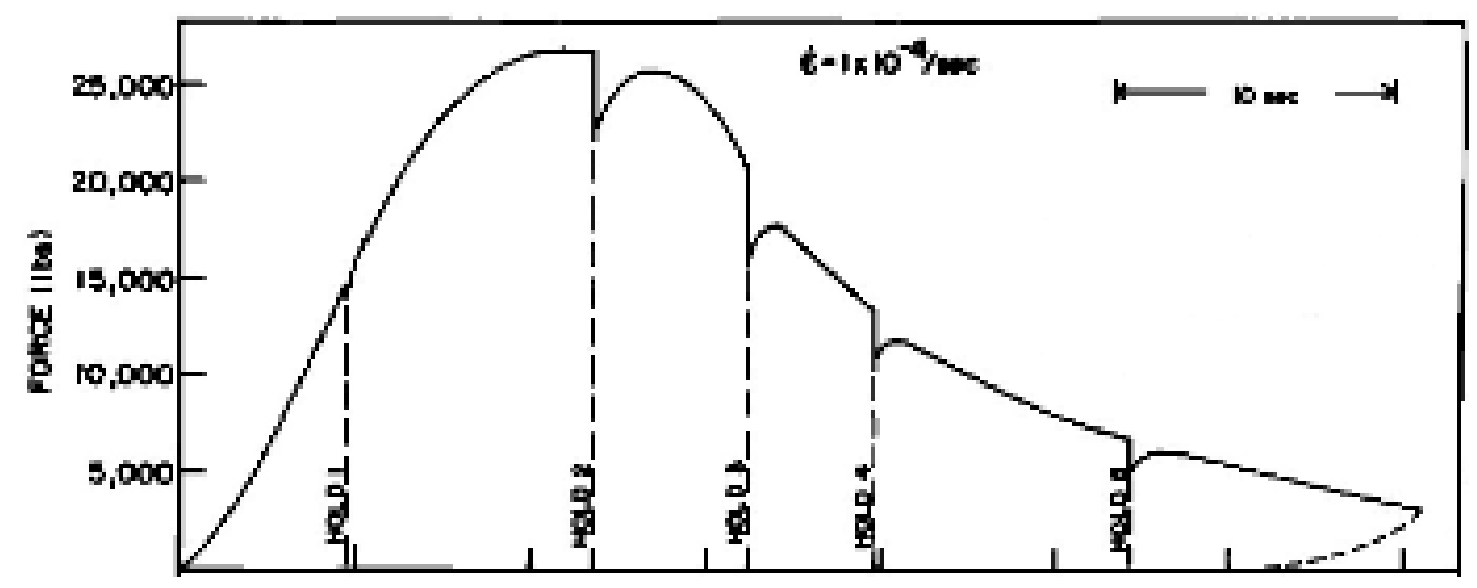

(a) The stress-strain curve with relaxation tests during an unconfined compression test on marble

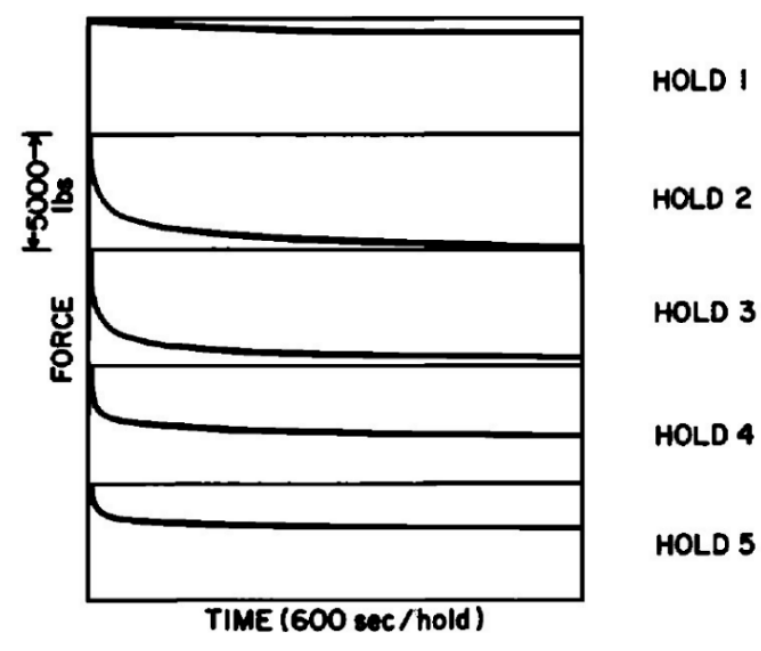

(b) Load-time curves for the relaxation tests

Fig. 3.2. Relaxation test results by Hudson and Brown (Hudson and Brown 1972)

Relaxation test, rather than creep test, is the preferred option in the study of the time-dependent property of rock. Various researchers have performed relaxation tests at different stages of a complete loaddisplacement curve (Hudson and Brown 1972; Peng and Podnieks 1972; Peng 1973), successfully overcoming the challenges posed in the constant load tests. The test results demonstrate that, at the same stress level, relaxation in the post-failure region is more pronounced than in the pre-failure region, as shown in Fig. 3.2. However, these studies only used the relaxation test results to qualitatively describe the stress 
relaxed from the specimens. They made no attempt to quantify the evolution of time-dependent property before and after failure. If the relaxation test results can determine the time-dependent parameters, it should be possible to represent the variation in the time-dependent property of rocks before and after failure by the varying time-dependent parameters determined at different stages of a complete stress-strain curve with the same constitutive model in the pre-failure and post-failure region. Therefore, it is imperative to investigate the variation in time-dependent property with relaxation test.

This chapter contains the development of a relaxation model based on Burgers model. Laboratory tests, including creep test and relaxation test, were conducted on rock specimens in the pre-failure region to verify the method of using relaxation test to determine the time-dependent parameters. Due to the complicity of the equation, the procedure to determine the associated parameters from laboratory relaxation test results were also proposed. In addition, some relaxation tests were conducted in the post-failure region. The expectation was to determine the time-dependent parameters of failed rock with the relaxation model by assuming viscoelastic behaviors in the post-failure region. However, the axial-displacement control model failed to capture the strain-softening process.

\subsection{DEVELOPMENT OF A RELAXATION MODEL}

\subsubsection{Relation between creep and relaxation}

Spence and Hult (2001) defined creep as a state caused by prescribed boundary forces while relaxation represents the state caused by prescribed boundary displacements. Under unconfined conditions, creep refers to continuously increasing strain with fixed boundary forces, while relaxation refers to the drop in the stress with fixed boundary deformation. This demonstrates that creep and relaxation are two intrinsic time-dependent behaviors of material caused by different boundary conditions. In other words, the intrinsic time-dependent properties of the material should be consistent, no matter whether investigated with a creep test or a relaxation test.

Numerical simulation of unconfined relaxation test was then performed on a simulated rock specimen using Burgers material in 3DEC (Itasca 2012). The test kept the length to diameter ratio of the specimen with a height of $10 \mathrm{~m}$ and a diameter of $5 \mathrm{~m}$ was kept at 2:1. In addition, the model was meshed into tetrahedral zones with an average edge length of $0.4 \mathrm{~m}$. The vertical velocity of the model bottom was set to zero and a constant stress was applied on the top of the model. After the model reached equilibrium condition, the vertical displacement of the top of the model was then fixed, as shown in Fig. 3.3, and creep model was activated to run for six hours. A function written with FISH, a programming language embedded within 3DEC, was used to monitor the stress within the specimen. The resulted relaxation curve with parameters, $\sigma\left(\right.$ or $\left.\sigma_{0}\right)=55.12 \mathrm{MPa}, \quad v=0.25, E_{m}=413.4 \mathrm{GPa}, E_{k}=11.44 \mathrm{GPa}, \eta_{m}=6.61 \times$ $10^{6} \mathrm{GPa} \cdot s, \eta_{k}=2.4 \times 10^{5} \mathrm{GPa} \cdot s$, is plotted in Fig. 3.4. 


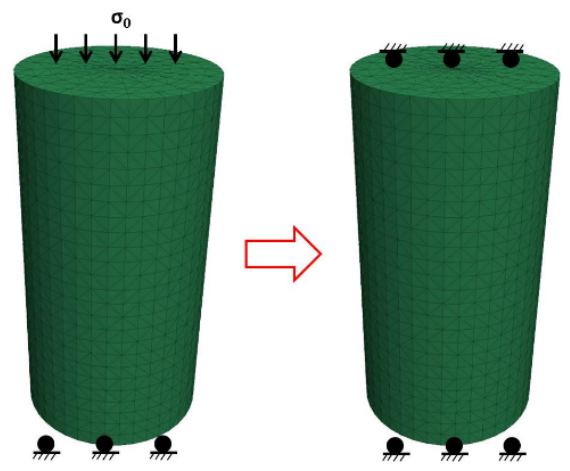

Fig. 3.3. Schematic for numerical simulation of unconfined relaxation test

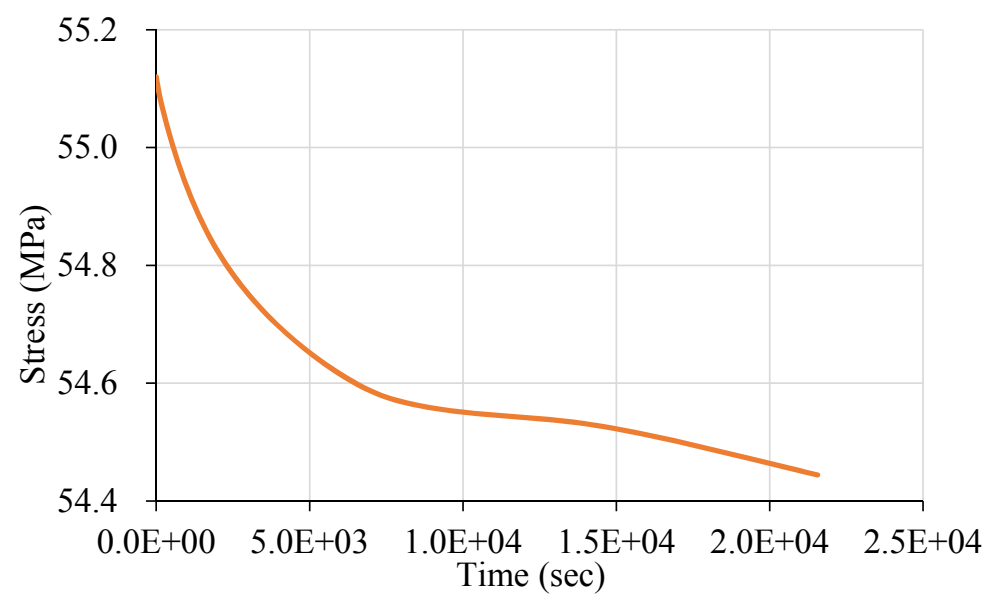

Fig. 3.4. Relaxation curve from numerical simulation

The simulation results demonstrate that, by changing the boundary condition, Burgers material can show relaxation behavior. As shown in Fig. 3.4, the stress within the specimen gradually decreased with time when the specimen was initially stressed, and the boundary displacement was fixed. In this simulation, the assignment of the parameters for Burgers model results in a relaxation curve. If such a relaxation curve is, in turn, obtainable from a laboratory relaxation test, it will be possible to determine the parameters for Burgers model.

\subsubsection{Relaxation model}

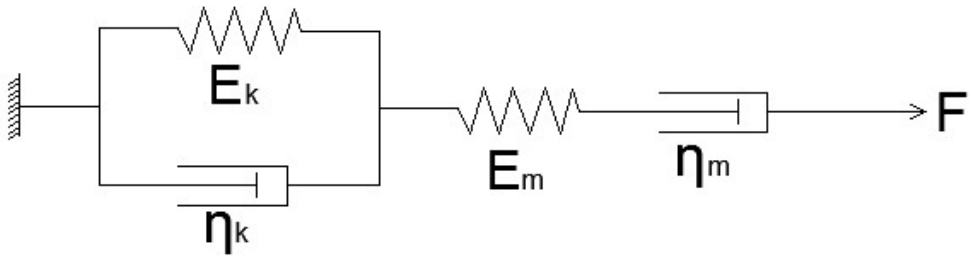

Fig. 3.5. Schematic of Burgers model

Starting from Burgers model, the corresponding relaxation model was derived in this section. Since studies commonly use Burgers model to represent the creep behavior of rocks (Price 1964; Fakhimi and Fairhurst 1994; Malan et al. 1997; Ghorbani and Sharifzadeh 2009; Li and Ghassemi 2012), it was used in the paper to describe the time-dependent behaviors of rock. Burgers model consists of one Kelvin unit and 
one Maxwell unit as shown in Fig. 3.5, where the subscripts, $\mathrm{k}$ and $\mathrm{m}$, represent that the parameters are for Kelvin unit and Maxwell unit, respectively. Eq. 3.1a and Eq. 3.1b show Burgers model for unconfined creep condition, where $\mathrm{K}$ is bulk modulus. In Eq. 3.1a, the first term of the right side is the volumetric strain, and the other three terms represent the deviatoric strain. Therefore, the material behaves as an elastic body under hydrostatic compression and as a Burgers material under deviatoric stress condition (Goodman 1989).

$$
\begin{aligned}
& \varepsilon=\frac{\sigma}{9 K}+\frac{\sigma}{3 E_{m}}+\frac{\sigma}{3 \eta_{m}} t+\frac{\sigma}{3 E_{k}}\left[1-\exp \left(\frac{-E_{k} t}{\eta_{k}}\right)\right] \\
& \varepsilon=\frac{1}{9 K} \sigma+\left\{\frac{1}{E_{m}}+\frac{1}{\eta_{m}} t+\frac{1}{E_{k}}\left[1-\exp \left(\frac{-E_{k} t}{\eta_{k}}\right)\right]\right\} \frac{\sigma}{3}
\end{aligned}
$$

In Eq. $3.1 \mathrm{~b}, 1 / \mathrm{K}$ is the reciprocal of bulk modulus and the term $\left\{\frac{1}{E_{m}}+\frac{1}{\eta_{m}} t+\frac{1}{E_{k}}\left[1-\exp \left(\frac{-E_{k} t}{\eta_{k}}\right)\right]\right\}$ represents reciprocal of an apparent shear modulus. When time increases, the value of this term increases. The apparent shear modulus, which represents the resistance to deviatoric stress, gradually decreases with time. When the boundary displacement is fixed, the decreasing apparent shear modulus releases the stress within rock. From this point of view, a creep model can theoretically explain relaxation behavior.

For Kelvin unit,

$$
F=\dot{u}_{k} \eta_{k}+E_{k} u_{k}
$$

where $\dot{u}_{k}$ and $u_{k}$ are strain rate and strain of Kelvin unit, respectively, and the notation of $\mathrm{F}, E_{k}$ and $\eta_{k}$ can be found in Fig. 3.5. The derivative of $F$ to time, $t$, can be obtain from Eq. 3.2, as

$$
\dot{F}=\ddot{u}_{k} \eta_{k}+E_{k} \dot{u}_{k}
$$

For Maxwell unit,

$$
\dot{u}_{m}=\frac{\dot{F}}{E_{m}}+\frac{F}{\eta_{m}}
$$

where $\dot{u}_{m}$ is the strain rate of Maxwell unit and $\dot{F}$ is the stress-rate. The notation of $F, E_{m}$ and $\eta_{m}$ can be found in Fig. 3.5. It should be noted here that $E_{m}$ also represents shear modulus. When this model is describing the time-independent or instantaneous response corresponding to the situation that timestep is zero in numerical simulation, only the elastic part of Maxwell unit is active. Under this circumstance, Burgers model only shows elastic behavior described by $E_{m}$.

For relaxation, there is the following condition where the total strain increment is zero:

$$
\dot{u}_{k}+\dot{u}_{m}=0
$$

Substituting Eqs. 3.2 and 3.5 into Eq. 3.5, one can get,

$$
\left(\frac{1}{\eta_{m}}+\frac{1}{\eta_{k}}\right) F+\frac{1}{E_{m}} \dot{F}-\frac{E_{k}}{\eta_{k}} u_{k}=0
$$

Substituting Eqs. 3.2 and 3.3 into Eq. 3.6, there is now, 


$$
\frac{\eta_{k}}{E_{m}} \ddot{u}_{k}+\left(1+\frac{\eta_{k}}{\eta_{m}}+\frac{E_{k}}{E_{m}}\right) \dot{u}_{k}+\frac{E_{k}}{\eta_{m}} u_{k}=0
$$

which is a linear homogeneous second-order differential equation with constant coefficients. The general solution for this equation is,

$$
u_{k}=C_{1} \exp \left(r_{1} t\right)+C_{2} \exp \left(r_{2} t\right)
$$

where $C_{1}$ and $C_{2}$ are constants and,

$$
r_{1}, r_{2}=\frac{E_{m}}{2 \eta_{k}}\left[-\left(1+\frac{\eta_{k}}{\eta_{m}}+\frac{E_{k}}{E_{m}}\right) \pm \sqrt{\left(1+\frac{\eta_{k}}{\eta_{m}}+\frac{E_{k}}{E_{m}}\right)^{2}-4 \frac{E_{k} \eta_{k}}{E_{m} \eta_{m}}}\right]
$$

Substitute Eq. 3.8 into Eq. 3.5,

$$
F=C_{1}\left(r_{1} \eta_{k}+E_{k}\right) \exp \left(r_{1} t\right)+C_{2}\left(r_{2} \eta_{k}+E_{k}\right) \exp \left(r_{2} t\right)
$$

The initial condition indicates that $u_{k}=u_{k 0}$ and $F=F_{0}$ when $t=0$. With these initial conditions, the two constants are determined.

$$
\begin{aligned}
& C_{1}=\frac{F_{0}-u_{k 0}\left(E_{k}+r_{2} \eta_{k}\right)}{\left(r_{1}-r_{2}\right) \eta_{k}} \\
& C_{2}=\frac{u_{k 0}\left(E_{k}+r_{2} \eta_{k}\right)-F_{0}}{\left(r_{1}-r_{2}\right) \eta_{k}}
\end{aligned}
$$

If the history-dependence of Burgers model is ignored, the expression of $C_{1}$ and $C_{2}$ is,

$$
\begin{aligned}
C_{1} & =\frac{F_{0}}{\left(r_{1}-r_{2}\right) \eta_{k}} \\
C_{2} & =\frac{-F_{0}}{\left(r_{1}-r_{2}\right) \eta_{k}}
\end{aligned}
$$

Finally, the time-dependent stress during relaxation can be expressed as,

$$
F=F_{0}\left[\left(r_{1}+\frac{E_{k}}{\eta_{k}}\right) \exp \left(r_{1} t\right)-\left(r_{2}+\frac{E_{k}}{\eta_{k}}\right) \exp \left(r_{2} t\right)\right] /\left(r_{1}-r_{2}\right)
$$

The above calculation is for a one-dimensional situation. However, the purpose of this research is to derive the relaxation equation and in turn to find a method to determine the creep parameters from relaxation test data. What is obtainable from a relaxation test is the relation between vertical stress and time. As a result, it is necessary to express the relation between stress and time with a relaxation equation based on the above derivation.

During unconfined test, $\sigma$ is used to represent the axial stress. As such, one can express the deviatoric stress in the axial direction as,

$$
F=\frac{2}{3} \sigma
$$

Rather than the sum of Kelvin strain rate and Maxwell strain rate, expressed in Eq. 3.5, the total strain rate, including the elastic volumetric strain rate, should be zero. 


$$
\dot{u}_{k}+\dot{u}_{m}+\dot{u}_{v o l}=0
$$

where $\dot{u}_{v o l}$ represents the axial strain rate induced by volumetric strain, expressed as,

$$
\dot{u}_{v o l}=\frac{\dot{\sigma}}{9 K}
$$

where $K$ is the bulk modulus.

Following the calculating process from Eq. 3.6 through Eq. 3.15, one can derive the relaxation equation under unconfined condition as,

$$
\sigma=\sigma_{0}\left[\left(r_{1}+\frac{E_{k}}{\eta_{k}}\right) \exp \left(r_{1} t\right)-\left(r_{2}+\frac{E_{k}}{\eta_{k}}\right) \exp \left(r_{2} t\right)\right] /\left(r_{1}-r_{2}\right)
$$

where,

$$
r_{1}, r_{2}=\frac{1}{2 \eta_{k}\left(\frac{1}{E_{m}}+\frac{1}{3 K}\right)}\left[-\left(1+\frac{\eta_{k}}{\eta_{m}}+\frac{E_{k}}{E_{m}}+\frac{E_{k}}{3 K}\right) \pm \sqrt{\left(1+\frac{\eta_{k}}{\eta_{m}}+\frac{E_{k}}{E_{m}}+\frac{E_{k}}{3 K}\right)^{2}-4 \frac{E_{k} \eta_{k}}{\eta_{m}}\left(\frac{1}{E_{m}}+\frac{1}{3 K}\right)}\right]
$$

Comparing the relaxation relations determined from the numerical simulation and theoretical calculation, with same parameters, Fig. 3.6 plots the relaxation curves from numerical simulation and theoretical calculation and shows good agreement. This demonstrates the validity of the derived relaxation equation.

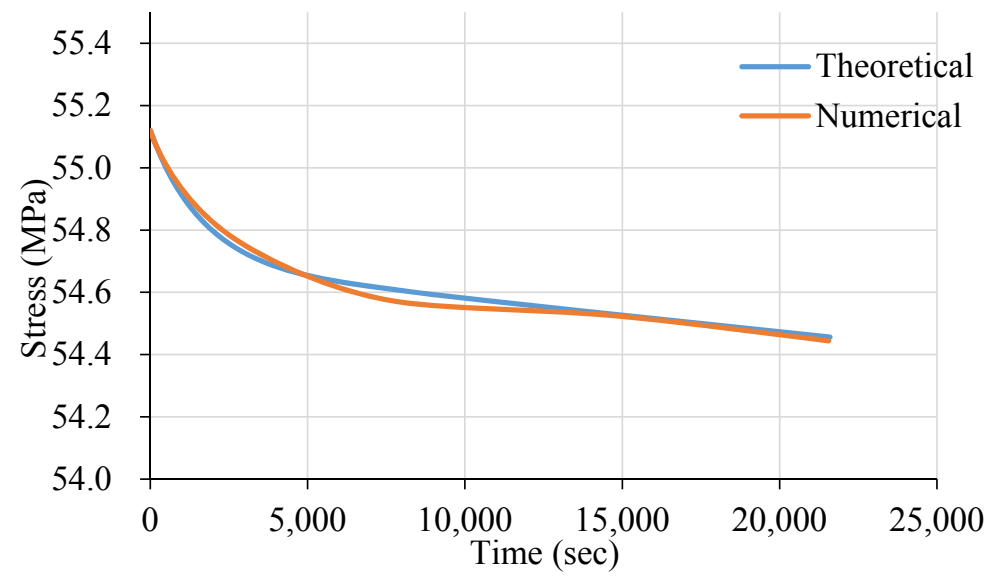

Fig. 3.6. Relaxation curve from numerical simulation

Finally, when comparing the creep behavior and relaxation behavior resulting from Burgers material using the same parameters in the numerical simulation, Eq. 3.1a and Eq. 3.19 provide the basis to plot the creep curve and relaxation curve, respectively. Comparing the two figures in Fig. 3.7 shows that similar to a creep curve, the relaxation curve also consists of two stages. Stress drop is rapid in the first stage, and in the second stage the stress drops with a nearly constant rate. In addition, it was likely that relaxation test could reach its secondary stage quicker than the creep test because the stress continues dropping during relaxation. However, the results show that the duration of the first stage of relaxation curve was similar to that of creep curve, where the difference is not noticeable. Therefore, the duration of a relaxation test in the 
pre-failure region should be the same as the time for a creep test.

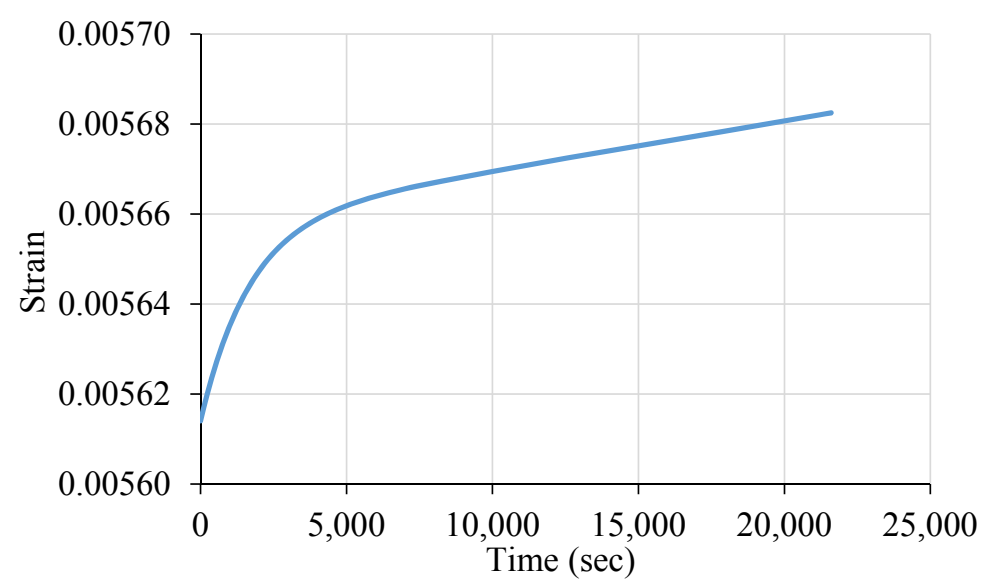

(a) Creep curve

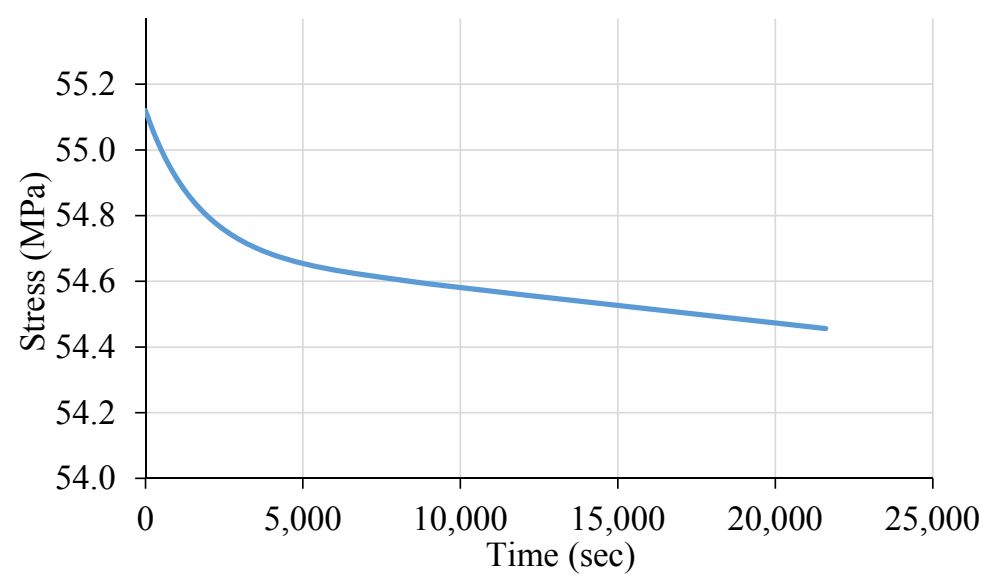

(b) Relaxation curve

Fig. 3.7. Theoretically calculated creep and relaxation curves with same parameters

\subsection{VERIFICATION OF USING RELAXATION TEST TO DETERMINE THE TIME-DEPENDENT PARAMETER}

This study conducted laboratory tests to investigate the creep behavior and relaxation behavior, as well as to verify the parameters determined from relaxation tests. First, the method of using relaxation test to study the time-dependent property of rock was to be verified in the pre-failure region. The theoretical analysis and numerical simulation in Section 3.1 verify the possibility of determining parameters for Burgers model with relaxation test. To verify this possibility in laboratory, the research approach was to perform various creep tests and relaxation tests on each specimen and then compare the parameters determined from different test methods. If the tests could achieve good agreement between creep test and relaxation test, relaxation test could then functionally study the time-dependent property of rock. Second, performing relaxation tests on specimens in the post-failure region attempted to study the time-dependent property of failed rock. If viscoelastic behavior is assumed for failed rock (Fakhimi and Fairhurst 1994), it is possible to extend this test method to the post-failure region. 


\subsubsection{Laboratory test design}

Three coal measure rocks, sandyshale, shaly limestone and limy shale, all obtained from Harrison County, West Virginia were used in the tests. The $50 \mathrm{~mm}(2 \mathrm{in}$.) core specimens were prepared as per recommended ASTM standard and were tested in a rig (MTS). Table 3.1 listed the property of the rocks tested. Two groups of laboratory test including a series of creep test and/or relaxation test at different stress or strain levels for each specimen.

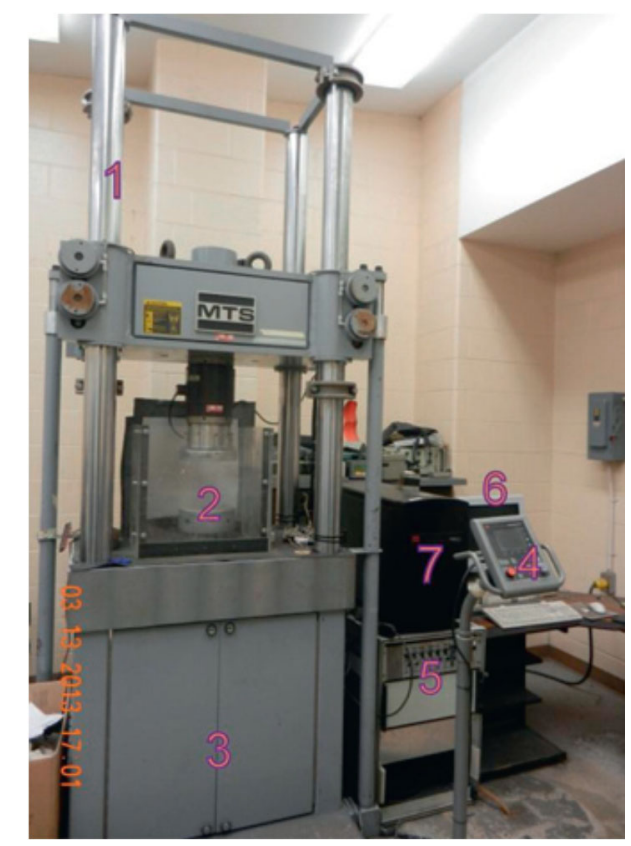

Fig. 3.8. MTS servo-controlled compression testing machine and its components

(1) Machine load, (2) Glass shield, (3) Hydraulic actuator, (4) Manual control system, (5) Strain Gauge control panel, (6) Computer, (7) MTS data acquisition system (Mishra and Verma 2015)

Table 3.1 Property of tested rocks

\begin{tabular}{|c|c|c|c|}
\hline Rock Type & Density $\left(\mathrm{kg} / \mathrm{m}^{3}\right)$ & Young's Modulus (GPa) & UCS (MPa) \\
\hline \multirow{3}{*}{ limy shale } & \multirow{3}{*}{2620} & 4.08 & 23.48 \\
\hline & & 3.66 & 22.03 \\
\hline & & 9.56 & 89.39 \\
\hline \multirow{3}{*}{ sandyshale } & \multirow{3}{*}{2624} & 12.13 & 101.61 \\
\hline & & 22.28 & 161.21 \\
\hline & & 14.03 & 104.94 \\
\hline \multirow{3}{*}{$\begin{array}{c}\text { shaly } \\
\text { limestone }\end{array}$} & \multirow{3}{*}{2658} & 17.21 & 82.49 \\
\hline & & 22.34 & 141.02 \\
\hline & & 19.14 & 89.37 \\
\hline
\end{tabular}

\section{(1) Group 1}

The purpose of the first group of tests was to verify the method of using relaxation test to study timedependent property of rock by comparing the parameters determined from creep test and relaxation test in the pre-failure region. Verifying this method requires a comparison of the results between two test methods. Because creep test is difficult to conduct on failed specimen, the comparison cannot be made in the postfailure region and as a result, the test was designed to compare the results in the pre-failure region. The 
procedure was to perform three creep tests and two relaxation tests for each specimen at different stress levels $(70 \%, 75 \%, 80 \%, 85 \%$ and $90 \%$ of UCS), as shown in Fig. 3.9. For each specimen, the parameters determined from the two relaxation tests and three creep tests can be averaged respectively and compared; for all the specimens in Group 1, the parameters are further averaged based on the test methods for the purpose of eliminating variation in the results caused by specimen heterogeneity. This group consists of tests on three specimens of shaly limestone.

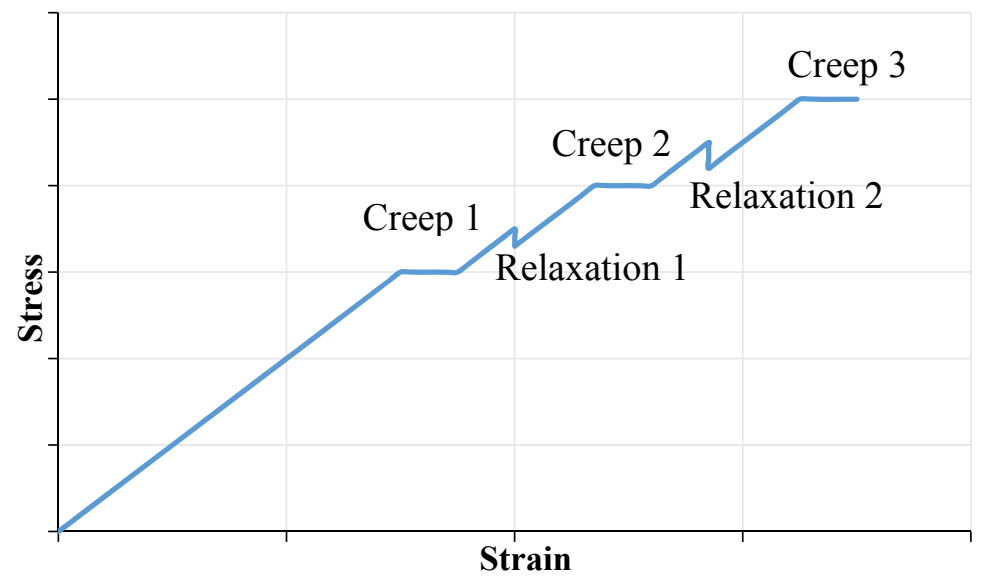

Fig. 3.9. Schematic of laboratory test procedure

\section{(2) Group 2}

The purpose of the second group of tests is twofold. For the second group of tests, two creep tests performed at two stress levels of the unconfined compression test on three specimens; next, five relaxation tests performed at different strain levels of the unconfined compression test on other three specimens of the same kind of rock. For the five relaxation tests, this study designed the first three to be conducted in the pre-failure region and compared with the creep test results. This is the first objective of the group of tests. This procedure tested sandyshale, limy shale and shaly limestone.

The second objective is to study the time-dependent property of failed rock. Previous relaxation tests on failed rock show more significant relaxation in the post-failure region than in pre-failure region. This signifies that failed rock can relax and transfer more stress to the surrounding rocks than intact rock. The last two of the five relaxation tests perform in the post-failure region to study the relaxation behavior of failed specimens. Comparing the relaxation curves from the pre-failure and post-failure region will then show the difference in the relaxation behavior between intact rock and failed rock. In addition, if the timedependent behavior is considered to be viscoelastic in the post-failure region, the same procedure can determine the model parameters of failed rock as in pre-failure region and, as a result, different parameters before and after failure can demonstrate the difference in time-dependent property. The model proposed by Fakhimi and Fairhurst (1994) assumed viscoelastic behavior for failed rock, but it did not consider the variation in the time-dependent property.

In summary, in Group 2, some specimens were tested with creep test in the pre-failure region while the others were tested with relaxation test in the pre-failure and post-failure region. The creep tests and 
relaxation tests in the pre-failure region can then verifty the method of using relaxation test to study the time-depedent property of rock. At the same time, the relaxation tests in the pre-failure and post-failure region can study the variation in the time-dependent property for intact and failed rock.

\subsubsection{Curve fitting procedure}

A fitting procedure is necessary to determine the viscous parameters from the results of the relaxation test. As seen in Eq. 3.20, the expression of unconfined relaxation behavior is complex. Fig. 3.10 shows an example of the relaxation curve obtained from laboratory test. It is difficult to determine the parameters by fitting the curve in Fig. 3.8 with Eq. 3.19 directly. Therefore, we proposed a fitting procedure to simplify this process.

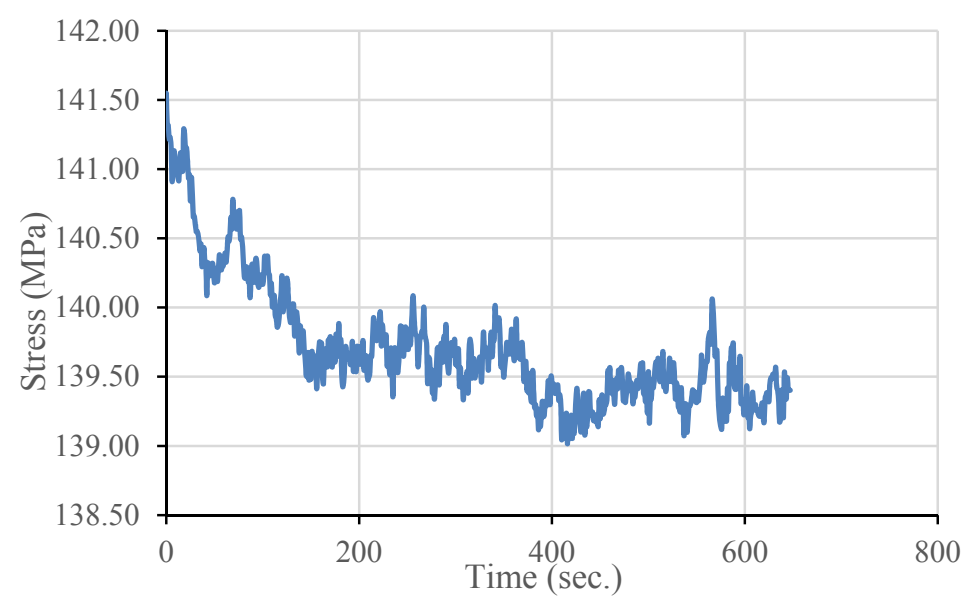

Fig. 3.10. Relaxation curve from laboratory test

If using three constants, $a, b$, and $c$, to represent $r_{1}, r_{2}$ and $\frac{E_{k}}{\eta_{k}}$, respectively, one can then write Eq. 3.19 as,

$$
\frac{\sigma}{\sigma_{0}}=[(a+c) \exp (a t)-(b+c) \exp (b t)] /(a-b)
$$

This equation describes the relation between stress ratio, $\frac{\sigma}{\sigma_{0}}$, and time, $t$, obtainable from laboratory test. Although the expression of $a, b$, and $c$ is complicated, they are constants in Eq. 3.21 and the laboratorydetermined stress-ratio-time relation can determine their values through curve fitting. As unconfined compression test can determine the value of bulk modulus and shear modulus, there are only three variables: $E_{k}, \eta_{k}$ and $\eta_{m}$. At the same time, there are three equations-the expressions of $a, b$, and $c$. Therefore, solving these three equations can determine the value of the three unknown creep parameters. For simplicity, the three variables are solved and expressed with $a, b$ and $c$ in the following three equations. Once the values are determined by curve fitting, one can directly substitute them into these equations to obtain the three viscous parameters for Burgers model.

$$
\begin{gathered}
\eta_{k}=\frac{-c}{(a+c)(b+c)\left(\frac{1}{E_{m}}+\frac{1}{3 K}\right)} \\
E_{k}=c \eta_{k}
\end{gathered}
$$




$$
\eta_{m}=\frac{-1}{\frac{1}{\eta_{k}}+(a+b+c)\left(\frac{1}{E_{m}}+\frac{1}{3 K}\right)}
$$

Software CurveExpert is a tool used to fit the laboratory test data to determine the above three constants. The laboratory data is modifiable within Excel to get the relation between time and $\frac{F}{F_{0}}$, as shown in Fig. 3.11. A user-defined model with the form of Eq. 3.21 (with three variables) was used for curve fitting, as shown in Fig. 3.12. After inputting the guessing value for the three variables, as shown in Fig. 3.13, the software can determine their values to get the best fitting result, as shown in Fig. 3.14. One can then substitute these three constants into Eq. 3.22 through Eq. 3.24 to obtain the three parameters for Burgers model.

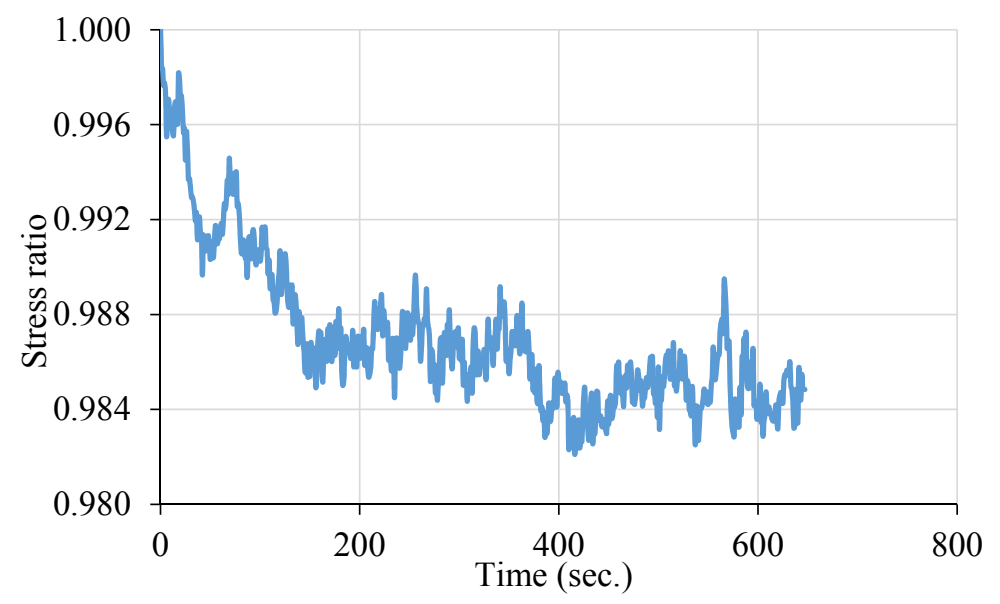

Fig. 3.11. Relation between time and $\mathrm{F} / \mathrm{F}_{0}$

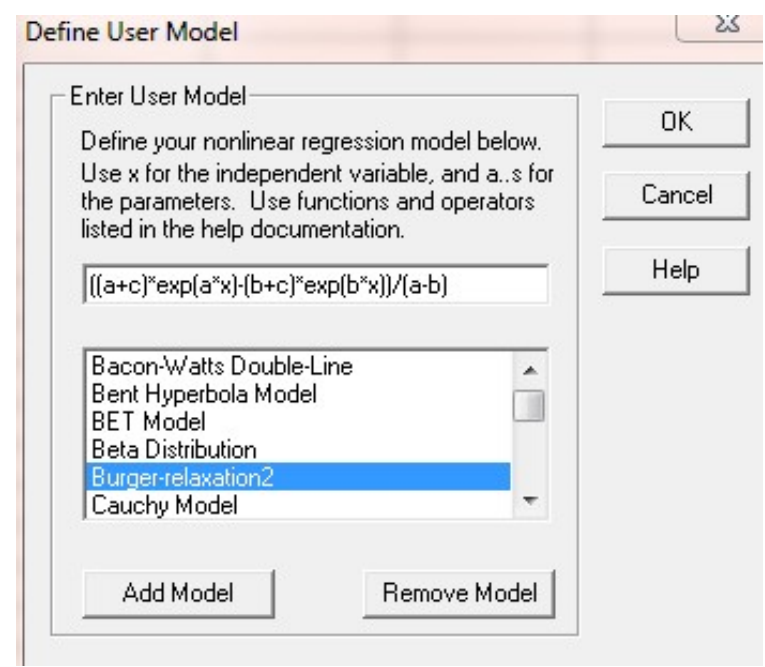

Fig. 3.12. User-defined mode in CurveExpert 


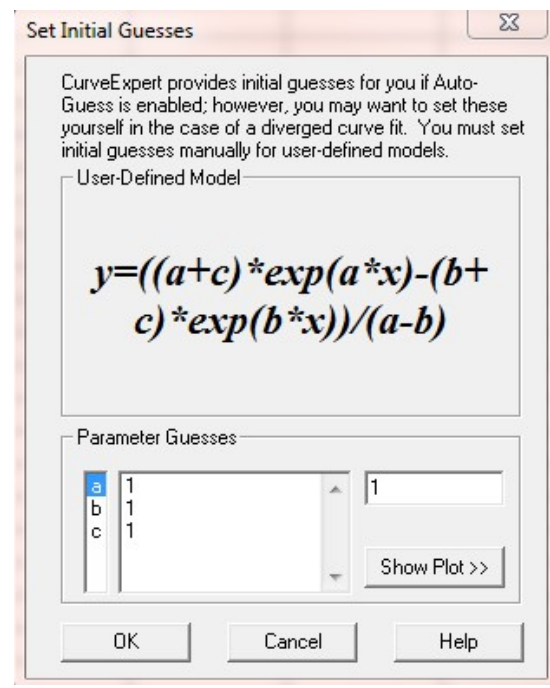

Fig. 3.13. Screen to input guess values for the three variables

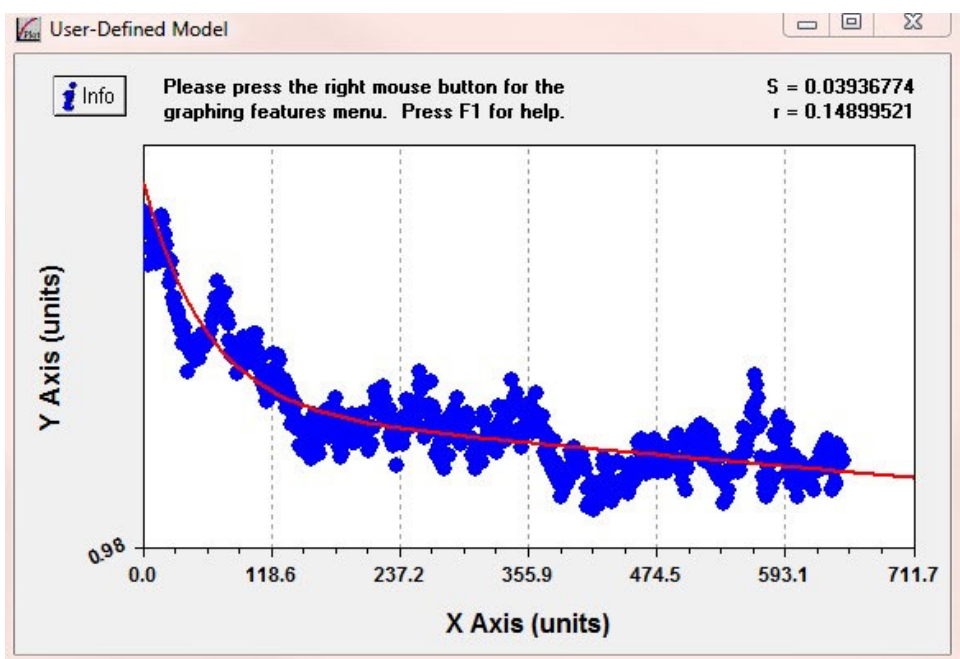

(a) Curve

Model Information - [User-Defined Model]

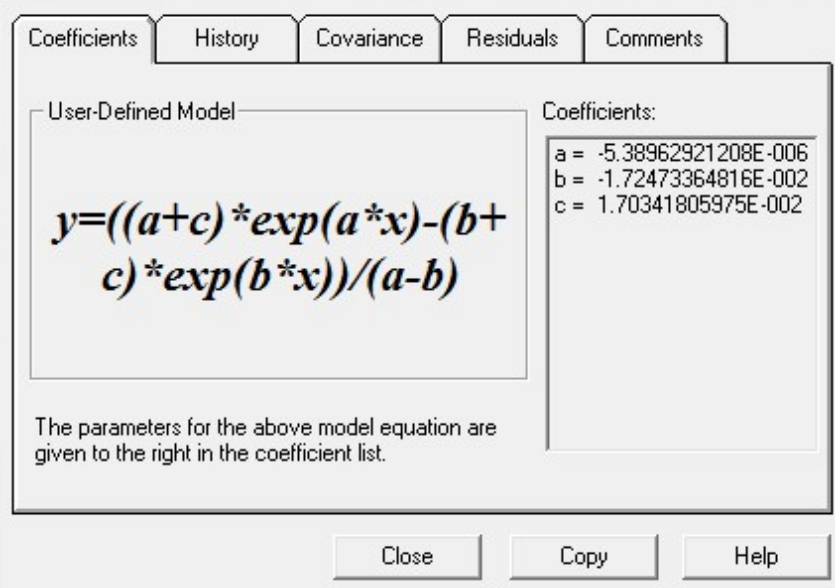

(b) Variable values

Fig. 3.14. Curve fitting result in CurveExpert 


\subsubsection{Test results and discussion}

The fitting procedure introduced by Goodman (1989) was used to determine the viscous parameters for Burgers model from the laboratory creep test. At the same time, relaxation tests can determine the viscous parameters based on the proposed procedure in this chapter. Figs. 3.15 and 3.16 show examples of the creep curves and relaxation curves determined from laboratory test and the fitted curves, respectively. The good agreement between the laboratory results and fitted models demonstrates that Burgers model can represent the time-dependent behaviors of rock for the test duration.

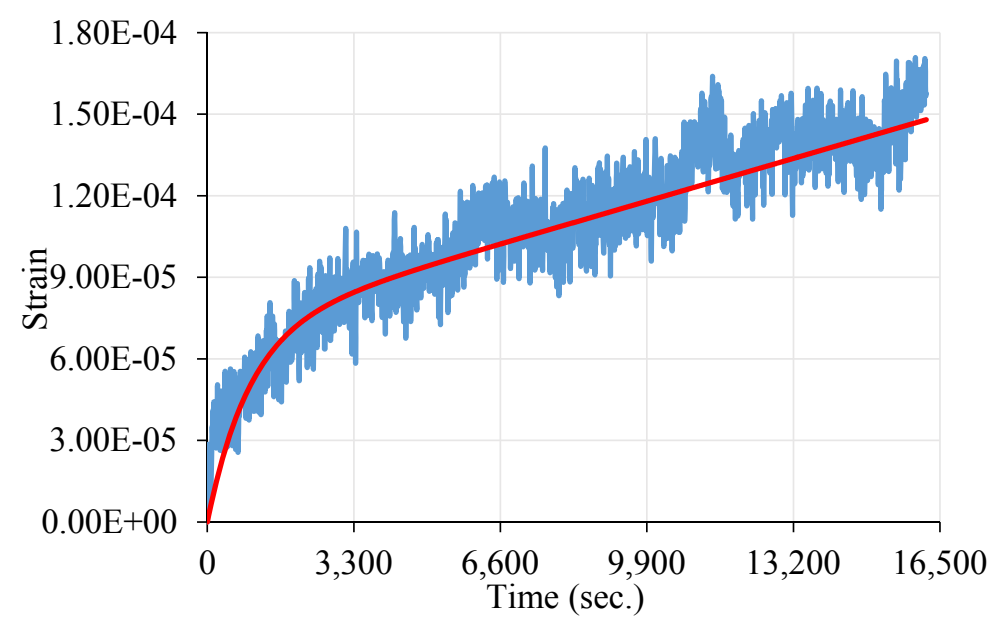

Fig. 3.15. Curve fitting result of Burgers model for creep test

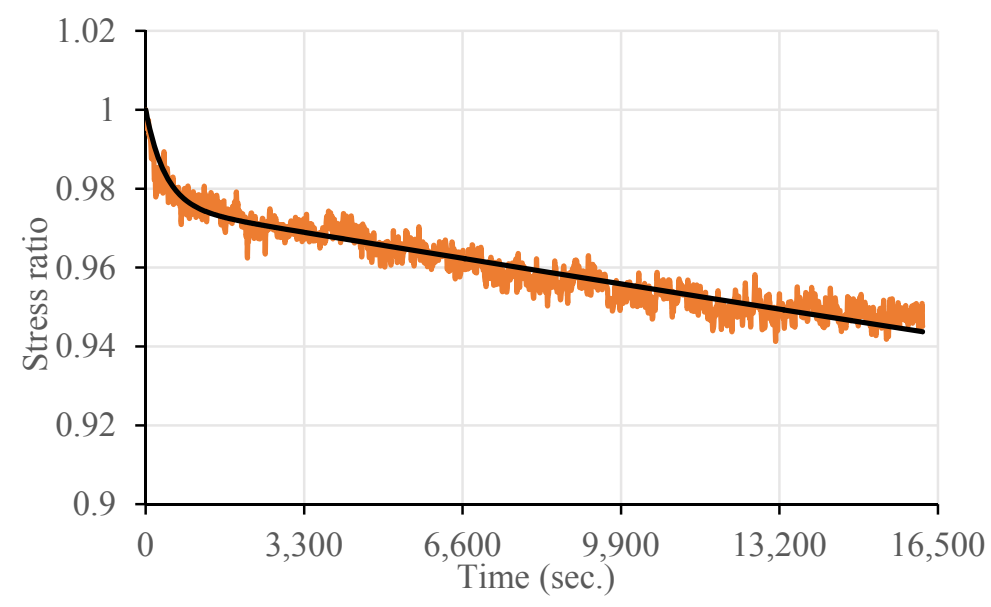

Fig. 3.16. Curve fitting result of Burgers model for relaxation test

Both creep tests and relaxation tests determined the viscous parameter for Burgers model for the three specimens of shaly limestone in Group 1, allowing the results to be compared. The bulk and shear modulus were calculated from the stress-strain curves before the first creep test. These values were then used to determine other parameters. Table 3.2 lists and averages the parameters determined from each creep test and relaxation test. For each specimen, the parameters were averaged based on the test method, and the results show a small variance in the averaged parameters between creep test and relaxation test. However, when further averaging the parameter over the three specimens, this minimizes the difference. The agreement between the parameters determined from different methods demonstrates that it is practicable to 
obtain viscous parameters from relaxation test.

Table 3.2 Comparison of creep parameters from creep and relaxation tests from first group of tests

\begin{tabular}{|c|c|c|c|c|c|c|c|c|c|}
\hline \multirow{2}{*}{ Specimen } & \multirow{2}{*}{ Stage } & \multirow{2}{*}{$\begin{array}{c}\text { K (psi) } \\
\text { Value }\end{array}$} & \multirow{2}{*}{$\frac{\text { Em }_{\mathbf{m}}(\mathbf{p s i})}{\text { Value }}$} & \multicolumn{2}{|c|}{$\eta_{\mathrm{m}}\left(\mathrm{psi}{ }^{*} \mathrm{~s}\right)$} & \multicolumn{2}{|c|}{$E_{k}(p s i)$} & \multicolumn{2}{|c|}{$\eta_{\mathrm{k}}\left(\mathrm{psi}{ }^{*} \mathrm{~s}\right)$} \\
\hline & & & & Value & Average & Value & Average & Value & Average \\
\hline \multirow{4}{*}{1} & Relaxation 1 & \multirow{4}{*}{$2.15 E+06$} & \multirow{4}{*}{$1.42 E+06$} & $4.87 \mathrm{E}+11$ & \multirow{2}{*}{$1.04 E+12$} & $5.46 \mathrm{E}+07$ & \multirow{2}{*}{$7.57 E+07$} & $6.36 \mathrm{E}+10$ & \multirow{2}{*}{$7.44 E+10$} \\
\hline & Relaxation 2 & & & $1.60 \mathrm{E}+12$ & & $9.67 \mathrm{E}+07$ & & $8.52 \mathrm{E}+10$ & \\
\hline & Creep 2 & & & $9.75 \mathrm{E}+11$ & $177 \mathrm{~F}+12$ & $4.80 \mathrm{E}+07$ & $516 \mathrm{~F} \perp 07$ & $4.53 \mathrm{E}+10$ & $56 \mathrm{~F} \perp 10$ \\
\hline & Creep 3 & & & $1.37 \mathrm{E}+12$ & $1.1 / \mathrm{E}+12$ & $5.51 \mathrm{E}+07$ & $5.10 \mathrm{E}+\mathrm{V} /$ & $4.59 \mathrm{E}+10$ & $4.56 \mathrm{E}+10$ \\
\hline \multirow{4}{*}{2} & Relaxation 1 & \multirow{4}{*}{$1.79 E+06$} & \multirow{4}{*}{$1.18 \mathrm{E}+06$} & $4.87 \mathrm{E}+11$ & \multirow{2}{*}{$8.59 \mathrm{E}+11$} & $8.96 \mathrm{E}+07$ & \multirow{2}{*}{$7.48 E+07$} & $7.23 \mathrm{E}+10$ & \multirow{2}{*}{$5.88 \mathrm{E}+10$} \\
\hline & Relaxation 2 & & & $1.23 \mathrm{E}+12$ & & $6.00 \mathrm{E}+07$ & & $4.52 \mathrm{E}+10$ & \\
\hline & Creep 2 & & & $5.95 \mathrm{E}+11$ & $752 \mathrm{~F}+11$ & $4.53 \mathrm{E}+07$ & $40 F+07$ & $3.79 \mathrm{E}+10$ & $260 F+10$ \\
\hline & Creep 3 & & & $9.11 \mathrm{E}+11$ & /. ОЈЕТ ПI & $4.26 \mathrm{E}+07$ & $4.40 \mathrm{ET} T \mathrm{~V}$ & $3.41 \mathrm{E}+10$ & 3.00ETIV \\
\hline \multirow{4}{*}{3} & Relaxation 1 & \multirow{4}{*}{$1.86 \mathrm{E}+06$} & \multirow{4}{*}{$1.23 E+06$} & $6.12 \mathrm{E}+11$ & \multirow{2}{*}{$9.01 E+11$} & $4.77 \mathrm{E}+07$ & \multirow{2}{*}{$4.49 E+07$} & $1.69 \mathrm{E}+10$ & \multirow{2}{*}{$1.72 \mathrm{E}+10$} \\
\hline & Relaxation 2 & & & $1.19 \mathrm{E}+12$ & & $4.21 \mathrm{E}+07$ & & $1.74 \mathrm{E}+10$ & \\
\hline & Creep 2 & & & $6.89 \mathrm{E}+11$ & \multirow{2}{*}{$8.00 E+11$} & $5.73 \mathrm{E}+07$ & \multirow{2}{*}{$7.30 \mathrm{E}+07$} & $1.96 \mathrm{E}+10$ & \multirow{2}{*}{$3.69 \mathrm{E}+10$} \\
\hline & Creep 3 & & & $9.10 \mathrm{E}+11$ & & $8.87 \mathrm{E}+07$ & & $5.41 \mathrm{E}+10$ & \\
\hline \multirow{2}{*}{ Average } & Relaxation & \multirow{2}{*}{$1.93 \mathrm{E}+6$} & \multirow{2}{*}{$1.28 \mathrm{E}+6$} & & $9.34 \mathrm{E}+11$ & & $6.51 \mathrm{E}+07$ & & $5.01 \mathrm{E}+10$ \\
\hline & Creep & & & & $9.08 E+11$ & & $5.62 \mathrm{E}+07$ & & $3.95 E+10$ \\
\hline
\end{tabular}

For the second group of tests, the tests did not follow the original design. Based on the test design, the first three relaxation tests were to be in the pre-failure region and the left two in the post-failure region. However, due to heterogeneity of the specimens, some specimens did not fail even after the fifth relaxation tests, while others failed before the second relaxation test. Therefore, the test number before and after failure does not match the design, and the following analysis only divides the test results into the pre-failure part and post-failure part.

The test results in pre-failure region are first analyzed. Fig. 3.17 through Fig. 3.19 show the fitted Burgers model parameters, $\eta_{m}, E_{k}$, and $\eta_{k}$. Each data point represents a "fitted" parameter from one creep test or relaxation test. The data points from creep test and relaxation test on the same type of rock have the same shape but differ in color. It is notable that the specimens' heterogeneity relatively separated the obtained parameters. However, the distribution of the data points demonstrates that some parameters determined from different test methods show good agreement, such as the value of $\eta_{m}$ for sandyshale and limy shale. Others show significant difference, such as the value of $E_{k}$ for all rocks. These can be confirmed by quantifying the distribution of these parameters with mean and standard deviation in Table 3.3. The large standard deviations indicate wide distribution of the fitted parameters. The mean values for certain parameters of certain rocks determined from creep test and relaxation test are very close, while others are quite different. This demonstrates the necessity of performing more tests to obtain more representative results. 


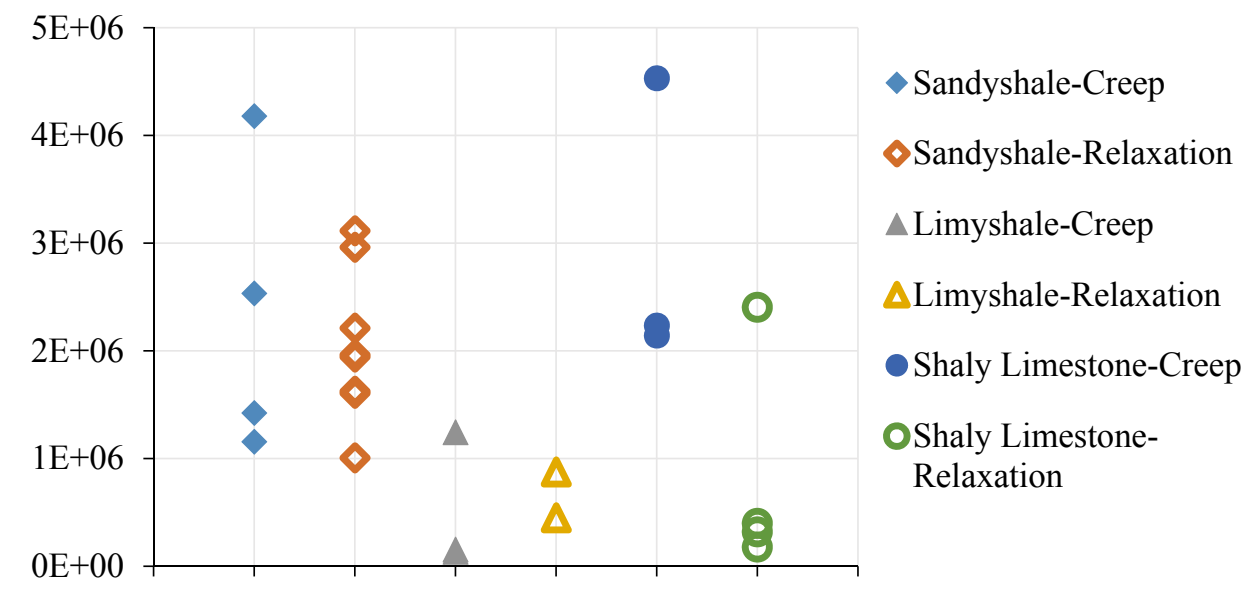

Fig. 3.17. Values of $\eta_{\mathrm{m}}\left(\mathrm{GPa}^{*} \mathrm{~s}\right)$ for different rocks with creep and relaxation tests from second group of tests

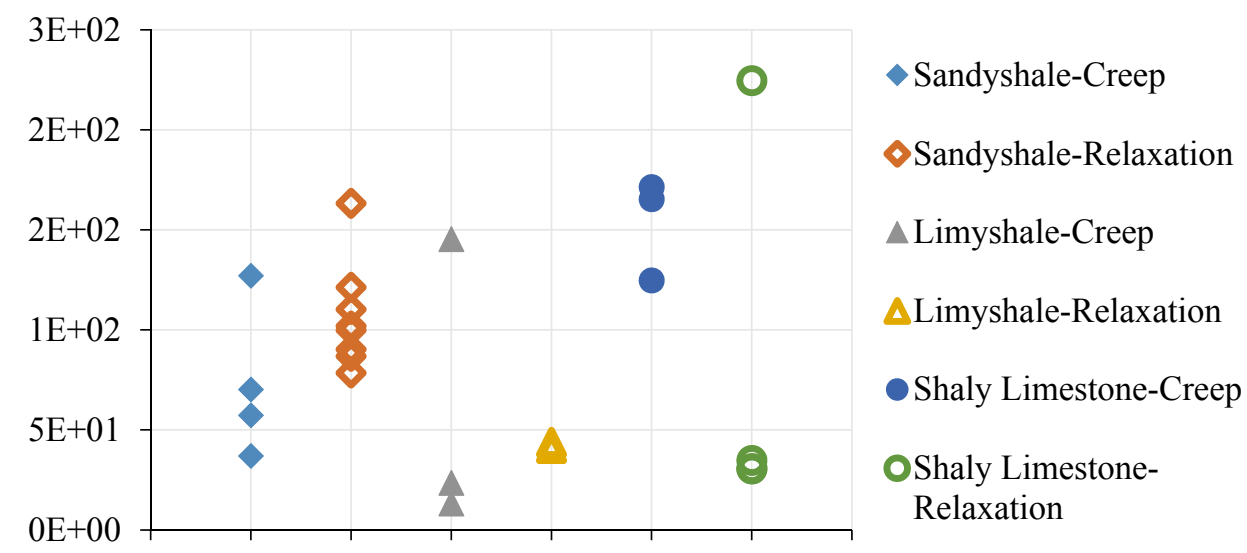

Fig. 3.18. Values of $E_{k}(\mathrm{GPa})$ for different rocks with creep and relaxation tests from second group of tests

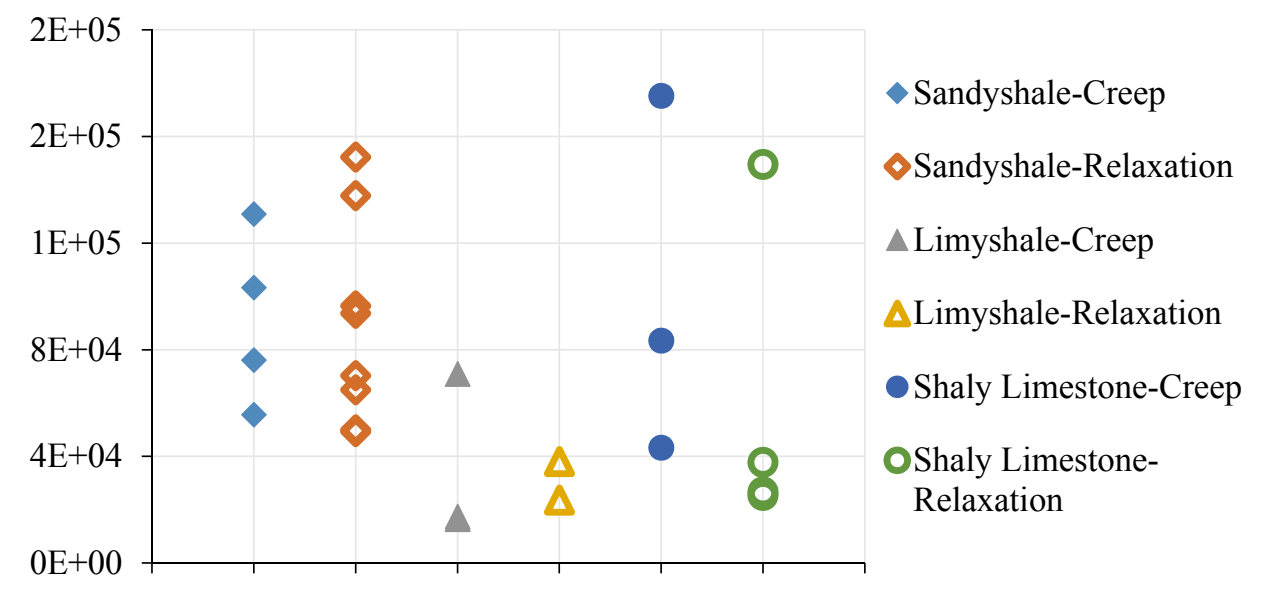

Fig. 3.19. Values of $\eta_{\mathrm{k}}\left(\mathrm{GPa}^{*} \mathrm{~s}\right)$ for different rocks with creep and relaxation tests from second group of tests 
Table 3.3 Mean and standard deviation of the viscous parameters

\begin{tabular}{|c|c|c|c|c|c|c|c|}
\hline \multirow{2}{*}{ Rock Type } & \multirow{2}{*}{$\begin{array}{c}\text { Test } \\
\text { Method }\end{array}$} & \multicolumn{2}{|c|}{$\eta_{m}(\mathbf{G P a} \mathbf{*})$} & \multicolumn{2}{c|}{$E_{k}(\mathbf{G P a})$} & \multicolumn{2}{c|}{$\eta_{k}(\mathbf{G P a} \mathbf{s})$} \\
\cline { 3 - 8 } & Mean & $\begin{array}{c}\text { Standard } \\
\text { Deviation }\end{array}$ & Mean & $\begin{array}{c}\text { Standard } \\
\text { Deviation }\end{array}$ & Mean & $\begin{array}{c}\text { Standard } \\
\text { Deviation }\end{array}$ \\
\hline \multirow{2}{*}{ sandyshale } & Creep & $2.32 \mathrm{E}+06$ & $1.19 \mathrm{E}+06$ & 72.92 & 33.43 & $9.15 \mathrm{E}+04$ & $2.84 \mathrm{E}+04$ \\
\cline { 2 - 8 } & Relaxation & $2.05 \mathrm{E}+06$ & $6.59 \mathrm{E}+05$ & 106.54 & 24.89 & $8.94 \mathrm{E}+04$ & $3.62 \mathrm{E}+04$ \\
\hline \multirow{2}{*}{ limy shale } & Creep & $5.10 \mathrm{E}+05$ & $5.18 \mathrm{E}+05$ & 60.76 & 59.98 & $3.50 \mathrm{E}+04$ & $2.54 \mathrm{E}+04$ \\
\cline { 2 - 8 } & Relaxation & $6.62 \mathrm{E}+05$ & $2.13 \mathrm{E}+05$ & 42.34 & 1.55 & $3.09 \mathrm{E}+04$ & $7.20 \mathrm{E}+03$ \\
\hline \multirow{2}{*}{$\begin{array}{c}\text { shaly } \\
\text { limestone }\end{array}$} & Creep & $2.97 \mathrm{E}+06$ & $1.35 \mathrm{E}+06$ & 153.88 & 25.38 & $1.01 \mathrm{E}+05$ & $6.77 \mathrm{E}+04$ \\
\cline { 2 - 8 } & Relaxation & $8.25 \mathrm{E}+05$ & $1.06 \mathrm{E}+06$ & 80.17 & 96.32 & $5.99 \mathrm{E}+04$ & $6.00 \mathrm{E}+04$ \\
\hline
\end{tabular}

As discussed earlier, this study designed some relaxation tests to conduct in the post-failure region for the purpose of investigating the time-dependent property of failed rock. Based on the assumption that the time-dependent behavior in the post-failure region is viscoelastic, the result of relaxation test conducted in the post-failure region can determine the parameters for Burgers model. However, the testing system with axial-displacement control mode failed to capture the strain-softening process. The stress dropped almost directly from peak to residual value; as a result, no relaxation test data was available during strain-softening process. Even so, some relaxation tests were successfully conducted in the residual stage.

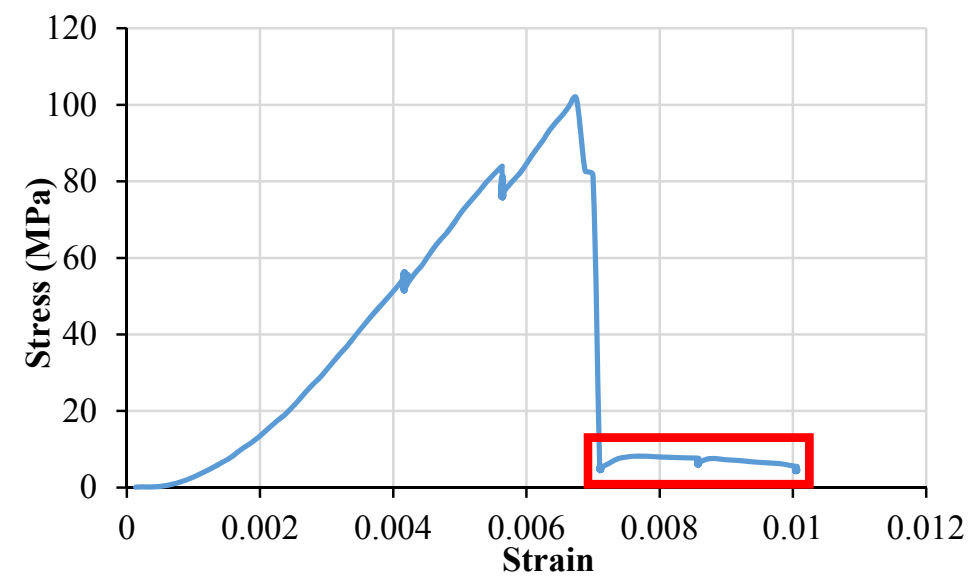

(a) Stress-strain curve

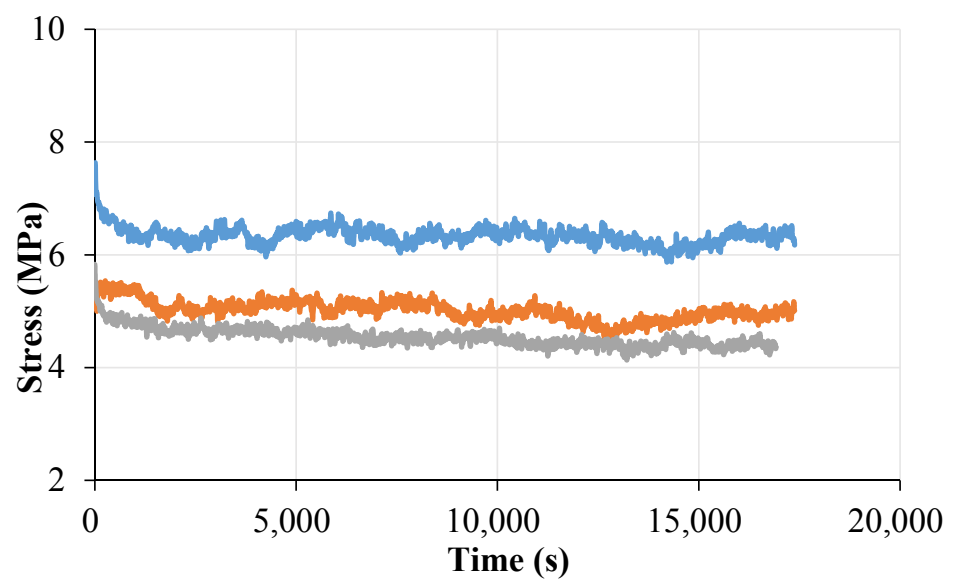

(b) Relaxation curves in the residual stage

Fig. 3.20. Relaxation tests in the residual stage 
The relaxation tests in the residual stage demonstrate significant relaxation for failed rock. For one specimen, the last three relaxation tests occurred in the residual stage, as shown in Fig. 3.20. The relaxation behavior in the residual stage is similar to that before failure where stress drop is quick at first and then gradually changes to a constant rate. In addition, the stress drop is significant during relaxation in the residual stage. The stresses at the beginning of the relaxation test were less than $10 \mathrm{MPa}$; however, they dropped by about $2 \mathrm{MPa}$ at the end of the relaxation tests. Around twenty percent of the stress relaxed within five hours. Although the magnitude of relaxed stress is relatively low, the ratio of the relaxed stress to the initial stress is very high.

In addition, the test of Group 2 showed one interesting phenomenon: failure occurrence during a relaxation test. As shown in Fig. 3.21, during the fourth relaxation test, the specimen failed after about 120 seconds of relaxation. The specimen did not fail when the relaxation test began, but instead failed when the stress was relaxed to a lower value. This implies that the strength reduced during relaxation test. In addition, the only process occurring during the relaxation test is the transfer of elastic strain to viscous strain. This indicates that the strength deterioration is the result of this conversion or the accumulated viscous strain (Fakhimi and Fairhurst 1994).

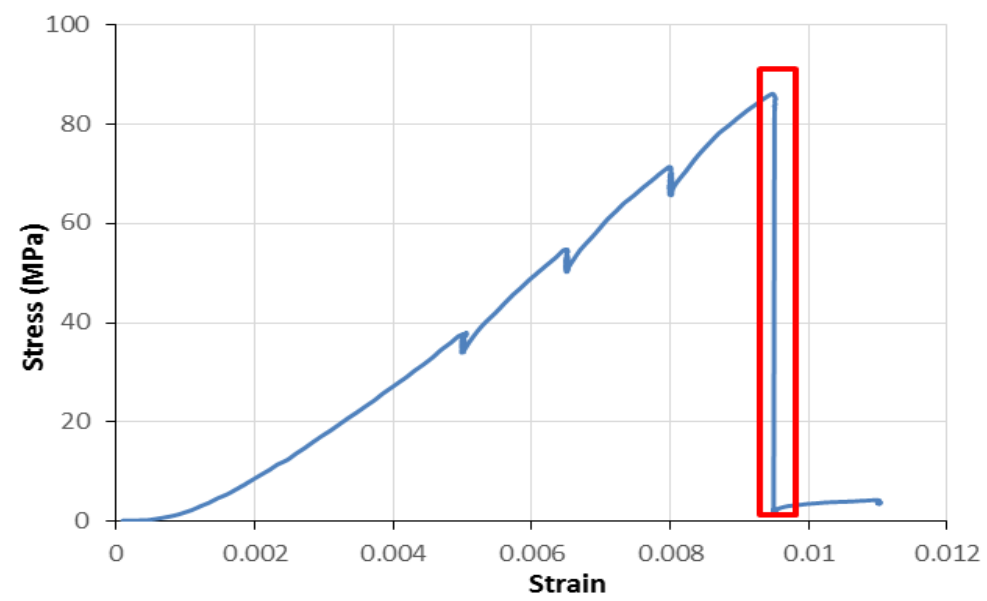

(a) Stress-strain curve

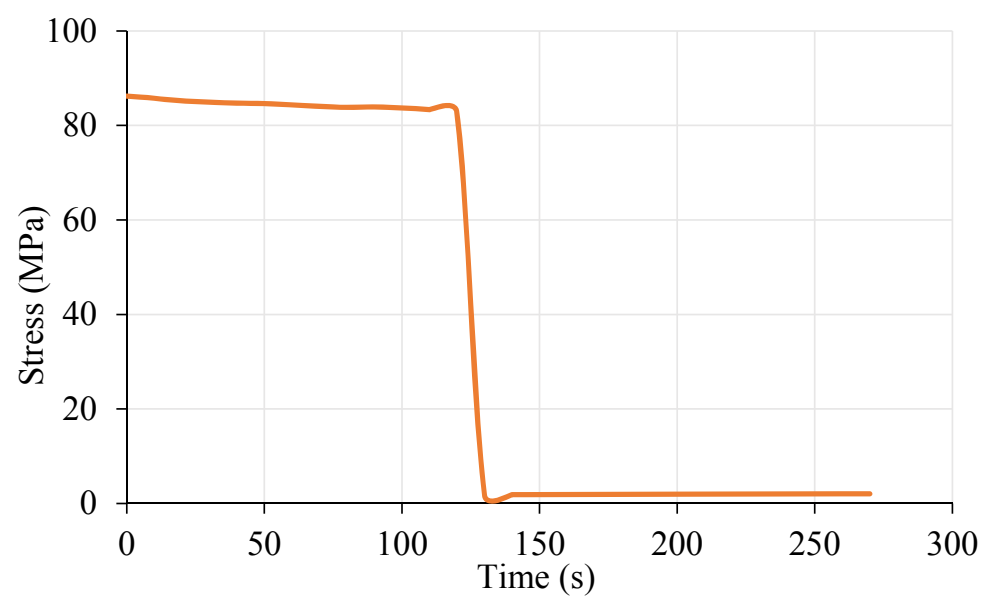

(b) Relaxation curve including failure occurrence

Fig. 3.21. Failure during a relaxation test 


\subsection{SUMMARY}

Roof falls usually occur after time-dependent roof convergence, studied previously from the viewpoint of rock creep. It is important to determine the creep parameters of roof rocks when investigating the timedependent behavior of underground mine roofs. However, creep test cannot be performed on failed specimens; thus, it cannot obtain the viscous parameters of failed rock. Laboratory relaxation test was proposed as an alternative method to determine the viscous parameters of rock before and after failure.

This study examined relaxation behavior theoretically and numerically with Burgers model. The results showed that the creep model was effective for describing relaxation behavior when boundary displacement was fixed; this indicates that the intrinsic time-dependent property of the material should be consistent, no matter if it is investigated with creep test or relaxation test. A relaxation equation for unconfined condition was then derived based on Burgers model and a detailed fitting procedure was described to determine creep parameters from relaxation test data.

In addition, two groups of laboratory tests ran in the pre-failure region to verify the proposed method. Multistage creep tests and relaxation tests were performed on rock specimens in the pre-failure region, one by one. The viscous parameters for Burgers model were determined from each test and were compared between the test methods. The results demonstrated that due to specimen heterogeneity, little variance existed between specimens; also, good agreement was obtained when the parameters were averaged over all the specimens of the same type of rock. Therefore, the relaxation test is a reliable method to determine the viscous parameters in the pre-failure region.

Finally, the study attempted to investigate the time-dependent behavior of failed specimens. As creep test is difficult to conduct on failed specimen, the expected solution was to extend the method of using relaxation test to determine the time-dependent parameters to the post-failure region. However, due to the failure of capturing strain-softening process, no relaxation tests ran successfully during strain-softening process. There were two interesting phenomena observed. First, the relaxation tests conducted in the residual stage showed more significant relaxation for failed rock than that of intact rock. Second, failure occurred during relaxation tests, demonstrating strength deterioration with viscous deformation. 


\section{CHAPTER 4 \\ THE INFLUENCE OF THE VARIATION IN THE TIME- DEPENDENT PROPERTIES AND STRENGTH DEGRADATION ON TIME-DEPENDENT ROOF FAILURE}

\subsection{INTRODUCTION}

The process of massive failure begins with localized failure that gradually progresses along the mine entry. When the failure reaches the competent, overlying strata, the entire roof rock collapses, exposing the overlying strata (Fig. 1.3). Therefore, one can call such failure a time-dependent failure.

Two types of behavior can result in this time-dependent failure of the roof: time-independent behavior and time-dependent behavior. The first type of behavior is not directly related to time, occurring after excavation and induced by the change in stress. Adjacent mining activities and rock failure within the roof can both affect the stress condition within the roof. For example, the development of adjoining entries, mining cut sequences, and movement of the longwall face will affect the local stress condition (Ray 2009). In addition, a roof fall or caving in nearby openings can also affect the stress condition, which can either promote or inhibit the failure propagation depending upon the condition (Gale et al. 1987; Aggson and Mouyard 1988; Dolinar et al. 2000a; Maleki et al. 2003). For example, the longwall face can reduce the horizontal stress with stress shadow, but it can also induce horizontal stress abutment. For these situations, numerical simulations usually ignore the influence of time.

The second type of behavior is directly relative to time. On one hand, the time-dependent response of the mine roof and the time-dependent behavior of the roof rocks can result in the time-dependent failure of the roof. The time-dependent deformation of roof rocks is one key factor contributing to the time-dependent roof convergence. The stress distribution within the roof changes along with the time-dependent deformation, potentially inducing failure propagation and further redistributing the stress. In addition, the variation in the time-dependent property between intact and failed rock potentially affects the stress distribution with time. Due to the variation in the time-dependent property, there will be a difference in the stress relaxation rate between intact and failed rock. If there is a failure zone initiated during the entry development within the roof, the stress relaxation behavior will be different inside and outside the failure zone; this will affect the stress distribution within the roof, especially the area close to the failure zone.

On the other hand, rock strength can deteriorate with time, possibly making a stable entry unstable over time. Failure of rock specimen in creep tests are known to occur at stress levels that are lower than the strength of the intact rock (Wawersik and Brown 1971; Baud and Meredith 1997; Rinne 2008). A creep test applies a lower stress than the rock strength onto a specimen. The specimen continues deforming and fails after some time, indicating that the rock strength degrades during the creep test. In other words, the strength degradation occurs with the increase in the time-dependent deformation. The time to failure under creep condition depends on the load, as shown in Fig. 4.1. In addition, shale, a common roof rock in underground 
coal mines, is known for its sensitivity to moisture (Van Eckhout 1976; Chungh and Missavage 1981; Masuda 2001; Molinda et al. 2004). The cyclic exposure of the shale roof rocks to moisture carried by ventilating air deteriorates the roof; as a result, the frequency of roof fall events changes with the season (Molinda et al. 2004). Other rocks can also deteriorate with the exposure to water (Heap et al. 2013). This is due to water's ability to promote the subcritical crack propagation within the specimens, deteriorating the rocks.

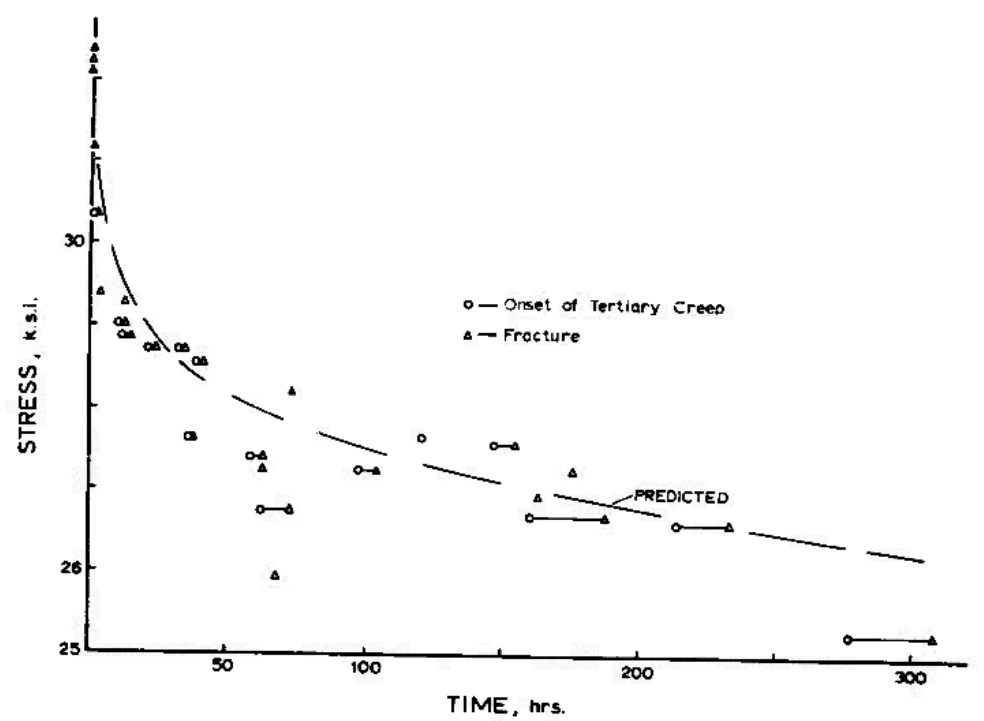

Fig. 4.1. Time to failure at different constant stress levels during the creep tests on Westerly Granite (Wawersik and Brown 1971)

The variation in the time-dependent property and strength degradation are two important factors in the study of time-dependent deformation and failure of mine roof. However, their influence on mine roof is not clear as of yet. If these two factors have a significant influence on the time-dependent roof stability, they need to be considered in the time-dependent simulation of mine roof. Otherwise, it is not necessary to incorporate them in the simulations. Incorporation of insignificant factors into the new model will only include more parameters into the new model. This section investigates the influences of the variation in the time-dependent property and strength degradation on the time-dependent failure within the mine roof separately. No new model was used in this section; instead, it uses Burgers model with Mohr-Coulomb failure criterion, referred as cvis model in $3 \mathrm{DEC}$, to represent the viscoelastic-plastic behavior of the roof rock. FISH functions were written to control the variation in the time-dependent property when failure occurs, as well as controlling the strength degradation with accumulated effective viscous strain. Comparing the simulation results with and without consideration of these factors will determine their influence on the time-dependent failure within mine roof.

\subsection{STUDY ON THE VARIATION IN THE TIME-DEPENDENT PROPERTY WITH RELAXATION TEST AND ITS INFLUENCE ON TIME-DEPENDENT ROOF FAILURE}

Chapter 3 verified the method of using relaxation test to determine the time-dependent parameters. If relaxation test is able to be conducted at different stages of a complete stress-strain curve, it can determine 
the time-dependent parameters at different stages; in turn, it can determine the variation in the timedependent property. However, the attempt to conduct the relaxation test failed due to the axial-displacement control mode failing to capture the strain-softening process. A few relaxation tests were performed in the residual stage, but not during strain softening process; instead, the relaxation tests from the literature (Hudson and Brown 1972) were used to study the variation in the time-dependent property and its influence. This study determined the time-dependent parameters in the pre-failure and post-failure region and compared them to demonstrate the variation in the time-dependent property. It then conducted numerical simulations to study the influence of the variation in the time-dependent property on the time-dependent roof behavior.

\subsubsection{Variation in the time-dependent property of rock}

Previous studies have not investigated the variation in the time-dependent property of rock, and overall understanding of the variation was lacking. In the pre-failure region, studies usually average creep parameters to describe creep behavior. However, the time-dependent parameters in the post-failure region are still unknown.

Due to the lack of available relaxation test data, Fig. 3.2 (b) shows the five relaxation curves used to study the variation in the time-dependent property. Fig. 3.2 shows relaxation in the post-failure region to be more significant than relaxation in the pre-failure region. Failed rock showed more relaxation than intact rock for the same duration, even though the relaxation test initiated from a lower stress level in the postfailure region than the relaxation test starting from a higher level in the pre-failure region. There are two options that can account for this increase in the significance of relaxation after failure. The first one is to use a viscoelastic model to describe the viscous behavior for intact and failed rocks; this can employ a decrease in the time-dependent parameters when failure occurs to account for the change in viscous behavior. The second one is to include a viscoplastic model into the viscoelastic model to account for the increase in significance of relaxation in the post-failure region. As the following simulations used Burgers model and Chapter 3 derived the relaxation equation for Burgers model, this study assumes that the timedependent behavior in the post-failure region is still viscoelastic. Therefore, this study uses Burgers model to describe the time-dependent behavior of rock before and after failure. The variation in the viscous parameters demonstrate the variation in the time-dependent property.

The variation in the time-dependent property has not been investigated in the past. It is unclear how the time-dependent property varies before and after failure. On one hand, the time-dependent parameters may vary with plastic strain, such as the variation of cohesion value with effective shear plastic strain in the strain-softening model. This signifies that the time-dependent parameters are constants before failure and vary with plastic strain in the post-failure region. If this is the situation, constant time-dependent parameters can be used in the pre-failure region and varying parameters with plastic strain can be used in the postfailure region. On the other hand, the variation in the time-dependent parameters could be small in the postfailure region, with constant parameters representing the time-dependent property of failed rock. This would mean that the time-dependent parameters only vary once when failure occurs. For this situation, intact rock can use one group of constant parameters, and failed rock can use the other group of constant parameters. 
From Fig. 3.2 (b), it is notable that the last four relaxation curves in the post-failure region have already reached their secondary stages while the relaxation curve in the pre-failure stage does not. It is reasonable to obtain parameters for Burgers model from the relaxation curves in the post-failure region. Each relaxation curve consists of data points of force and time, with their relation converting into the relation between stress ratio, $\sigma / \sigma_{0}$, and time. CurveExpert software (2010) performed curve fitting using the entered data for the purpose of determining the three constants, $a, b$, and $c$, in Eq. 3.21. Eqs. 3.22, 3.23, and 3.24 can finally determine the three time-dependent parameters by substituting in these three variables. Table 4.1 shows the parameters in the post-failure region, illustrating that the difference in each of the creep parameters between these four relaxation tests is small. As a result, it is possible to average each parameter over four relaxation tests and then use the averaged values for the viscous property of the failed rock.

Table 4.1 Parameters in the post-failure region determined from Hudson and Brown (1972) paper

\begin{tabular}{|c|c|c|c|}
\hline \\
\hline Parameter & Unit & Value & Averaged value \\
\hline $\mathbf{E}_{\mathbf{m}}$ & GPa & $2.65 \mathrm{E}+01$ & $2.65 \mathrm{E}+01$ \\
\hline \multirow{4}{*}{$\eta_{\mathrm{m}}$} & \multirow{4}{*}{ GPa *s } & $3.00 \mathrm{E}+05$ & \multirow{4}{*}{$2.74 \mathrm{E}+05$} \\
\hline & & $3.98 \mathrm{E}+05$ & \\
\hline & & $2.40 \mathrm{E}+05$ & \\
\hline & & $1.56 \mathrm{E}+05$ & \\
\hline \multirow{4}{*}{$\mathbf{E}_{\mathbf{k}}$} & \multirow{4}{*}{ GPa } & $1.16 E+02$ & \multirow{4}{*}{$8.99 \mathrm{E}+01$} \\
\hline & & $8.41 \mathrm{E}+01$ & \\
\hline & & $9.03 E+01$ & \\
\hline & & $6.96 \mathrm{E}+01$ & \\
\hline \multirow{4}{*}{$\eta_{\mathrm{k}}$} & \multirow{4}{*}{ GPa *s } & $1.63 E+03$ & \multirow{4}{*}{$1.24 \mathrm{E}+03$} \\
\hline & & $1.09 \mathrm{E}+03$ & \\
\hline & & $1.29 \mathrm{E}+03$ & \\
\hline & & $9.37 E+02$ & \\
\hline
\end{tabular}

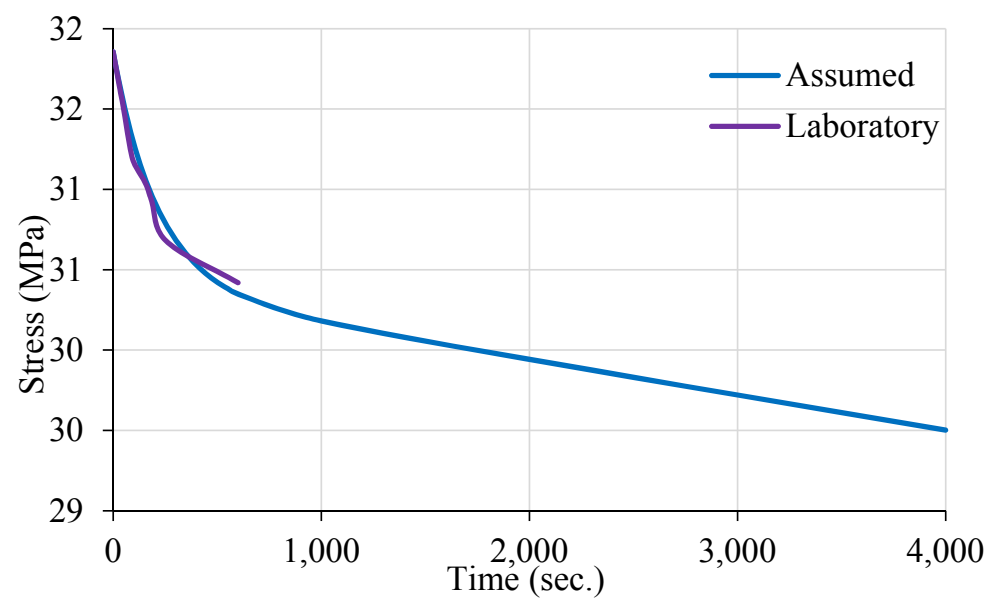

Fig. 4.2. Comparison of relaxation curves

In addition, the parameters for Burgers model in the pre-failure region are unavailable. There is only one relaxation test in the pre-failure region, and its duration is too short for the relaxation behavior to reach the secondary stage. However, the observation that relaxation in the post-failure region is more pronounced 
than in the pre-failure region indicates that the time-dependent parameters in the pre-failure region are larger than those in the post-failure region. In order to get the parameters in the pre-failure region, the parameters from the post-failure region were gradually increased to fit the relaxation curve marked as HOLD 1 in Fig. 3.2 (b). Fig. 4.2 compares the calculated relaxation curves with the assumed parameters and with HOLD 1. This figure shows that the relaxation curve with assumed parameters fits comparably well with the relaxation curve from laboratory test.

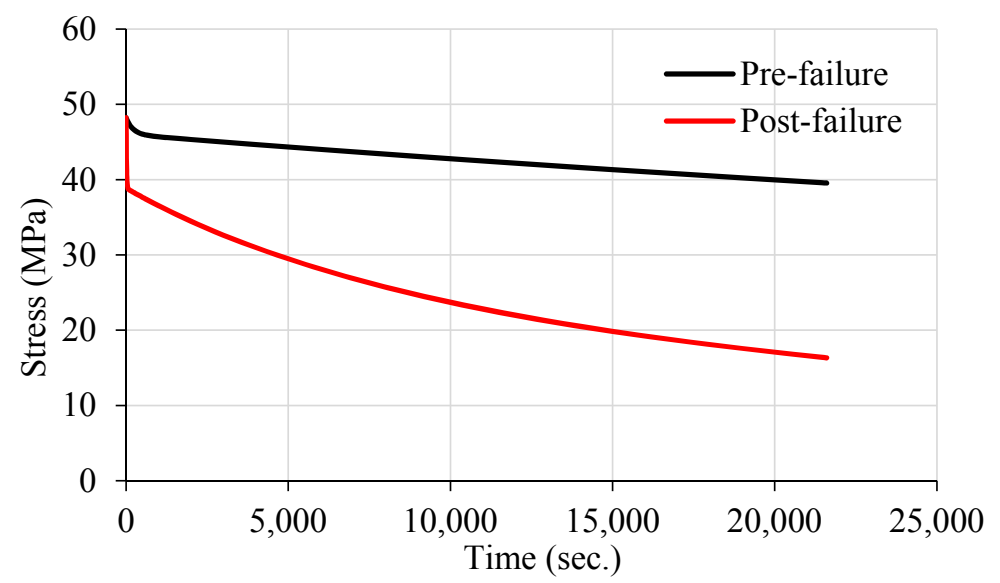

Fig. 4.3. Comparison of relaxation curves, starting from same stress values, before and after failure

Table 4.2 Parameters used in simulations

\begin{tabular}{|c|c|c|c|}
\hline \multicolumn{3}{|c|}{ Parameter } & Value \\
\hline \multirow{6}{*}{ Time-Independent } & \multicolumn{2}{|c|}{ Density $(\mathrm{Kg} / \mathrm{m} 3)$} & 2563 \\
\hline & \multicolumn{2}{|c|}{ Bulk Modulus (GPa) } & 40.17 \\
\hline & \multicolumn{2}{|c|}{ Shear Modulus/Mshear (GPa) } & 26.46 \\
\hline & \multicolumn{2}{|c|}{ Cohesion (MPa) } & 8.34 \\
\hline & \multicolumn{2}{|c|}{ Friction Angle (degree) } & 30.00 \\
\hline & \multicolumn{2}{|c|}{ Tensile Strength (MPa) } & 6.89 \\
\hline \multirow{6}{*}{ Time-Dependent } & \multirow{3}{*}{ Pre-Failure } & Mviscosity $\left(\mathrm{GPa}^{*} \mathbf{s}\right)$ & $2.74 \mathrm{E}+06$ \\
\hline & & Kshear (GPa) & $4.51 \mathrm{E}+02$ \\
\hline & & Kviscosity $\left(\mathrm{GPa}^{*} \mathbf{s}\right)$ & $9.92 \mathrm{E}+04$ \\
\hline & \multirow{3}{*}{ Post-Failure } & Mviscosity $\left(\mathrm{GPa}^{*} \mathrm{~s}\right)$ & $2.74 \mathrm{E}+05$ \\
\hline & & Kshear (GPa) & $9.03 \mathrm{E}+01$ \\
\hline & & Kviscosity (GPa*s) & $1.24 \mathrm{E}+03$ \\
\hline
\end{tabular}

Fig. 4.3 presents the relaxation curves plotted with different viscous parameters for intact and failed rock. The relaxation curves for conditions with smaller parameters in the post-failure region and larger parameters in the pre-failure region initiates at the same stress level. Fig. 4.3 illustrates that the difference in the viscous parameters results in significant differences in the overall trend of the relaxation curves. No matter in the first stage or the second stage, smaller viscous parameters produce larger relaxed stress for the same duration. In addition, the difference in magnitude of relaxed stress increases with time. Finally, numerical simulations were conducted on laboratory scale rock specimens using the parameters determined for intact and failed rock in the next section. Table 4.2 lists the parameters used in the simulations. 


\subsubsection{Simulation methodology to study the influence of variation in the time-dependent property}

This study performed numerical simulations using the commercial geotechnical software-threedimensional distinct element code (3DEC). Although designed for discontinuous medium, it can also simulate continuous medium. The model is meshed into finite-difference zones, with each zone behaving according to a prescribed linear or nonlinear stress-strain law. The explicit solution scheme used in 3DEC makes it possible to model complex and nonlinear behaviors. Burgers model, associated with MohrCoulomb failure criterion, is a built-in model in 3DEC that can run directly within the software.

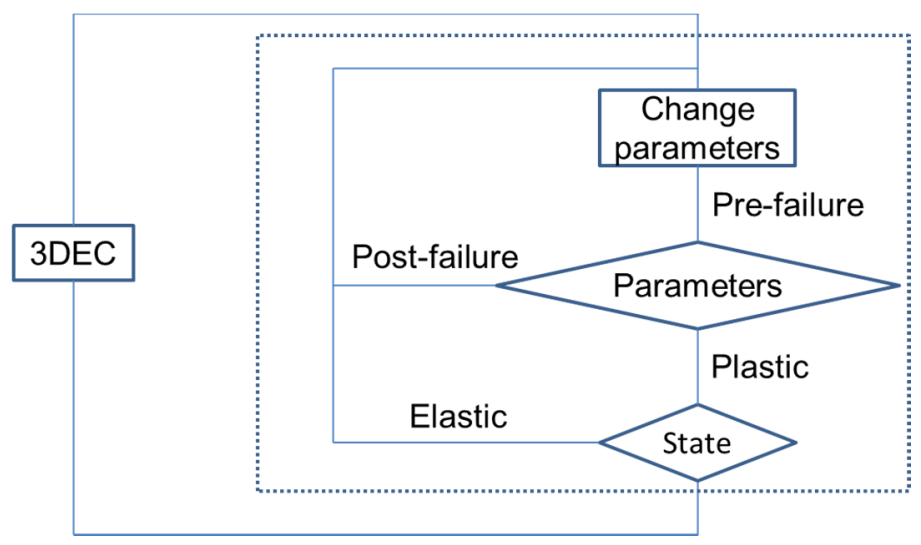

Fig. 4.4. Flowchart showing communication between 3DEC and FISH function

In addition, the application of FISH functions extends 3DEC's usefulness and adds user-defined features. FISH is a programming language embedded within 3DEC that enables the user to define new variables and functions (Itasca 2012) . During the simulations in this chapter, a FISH function monitored the zone state and changed the three time-dependent parameters when detecting zone failure. FISH function, $z_{-}$state (zi), monitored the state for each zone, where $z \boldsymbol{i}$ is the zone ID. Fig. 4.4 shows the calculating process with the FISH function. At the beginning of each timestep, function, $z$ _state (zi), scans all the zones within the model. After detecting plastic state, FISH function, z prop (zi, name), changed each parameter for the failed zones where name is the property name which is the same as the property name to assig properties with $\boldsymbol{Z O N E}$ command. After changing the parameters, the program maintains them for the following calculations even as the associated zones are unloaded. This accomplished the variation in the time-dependent property in these simulations within 3DEC.

In this section, two unconfined compression tests, each with one relaxation test in the pre-failure region and one in the post-failure region, were simulated with 3DEC. Fig. 4.5 shows the model. The bottom of the model was fixed, and a velocity boundary was applied from the top. The first simulation involves one unconfined compression test to determine the number of steps to failure. The next two simulations were two unconfined compression tests with relaxation tests. The simulation initially applied a velocity boundary from the top to a determined timestep in the pre-failure region; it then fixed the top boundary of the specimen to simulate relaxation test with duration of one hour. Afterwards, the simulation changed the top boundary to velocity boundary again to apply load to the post-failure or perfectly-plastic range. Finally, it performed the second relaxation test. The stress, strain, and creep time were monitored during this process. 
The two relaxation tests in the pre-failure region and in the post-failure region initiated at the same stress level. The difference between these two unconfined compression tests is the time-dependent parameters. One specimen was assigned with constant parameters which correspond to the values from the pre-failure region; the parameters for the other specimen varied with the method shown in Fig. 4.4. Figs. 4.6 and 4.7 show the resulting stress-strain curves and relaxation curves, respectively.

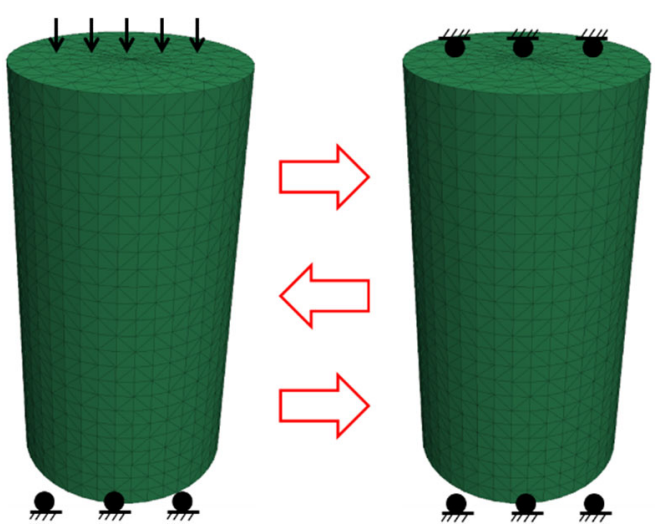

Fig. 4.5. Model for unconfined compression test with relaxation tests

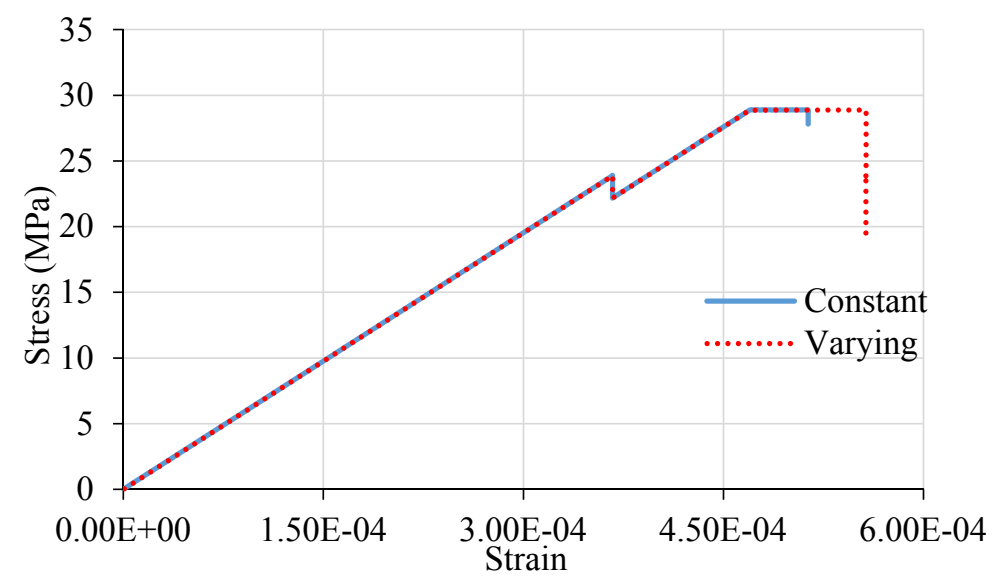

Fig. 4.6. Stress-strain curve for unconfined compression tests with relaxation test

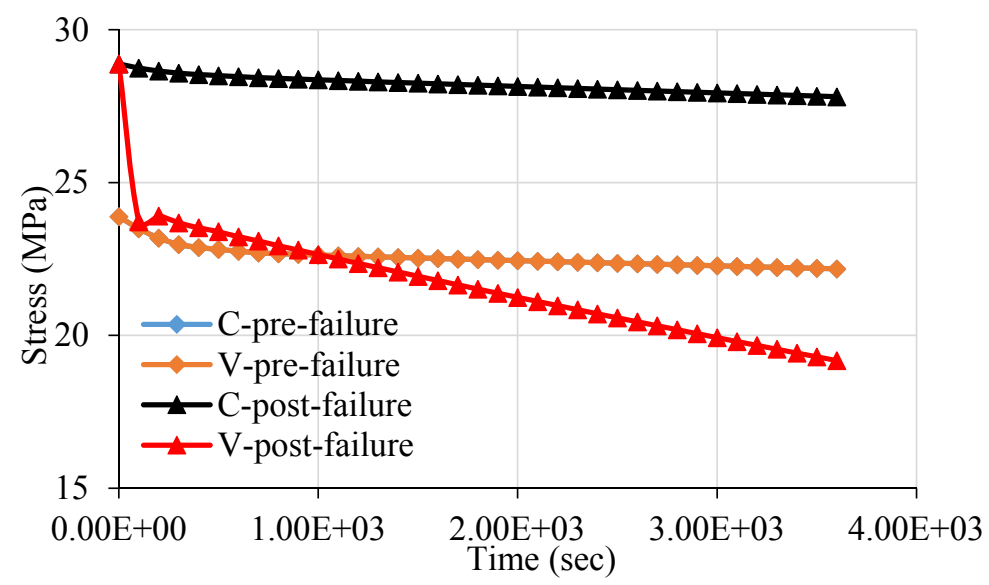

Fig. 4.7. Relaxation curves for the four relaxation tests.

'C' represents constant viscous parameters while "V" represents varying viscous parameters 
As seen in Figs. 4.6 and 4.7, the behaviors of two models are the same in the pre-failure region and are different in the post-failure region. The stress-strain curves in Fig. 4.6 and the relaxation curves in Fig.4.7 are nearly identical in the pre-failure region, indicating that the time-independent and time-dependent behaviors are the same for the rocks before failure. This is due to the use of the same parameters in these two models in the pre-failure region. However, when changing the three time-dependent parameters in the post-failure region for one model, different behaviors occurred in the post-failure region. Figs. 4.6 and 4.7 show that the specimen with varying viscous parameters relaxed more stress than that with constant parameters for the same period in the post-failure region. This signifies that the relaxation behavior in the post-failure region is more significant than in the pre-failure region. These two test simulations demonstrate that the proposed method successfully changed the parameters when detecting zone failure during cycling, resulting in different time-dependent behaviors in the post-failure region.

This study investigated the influence of the variation in the time-dependent property by simulating an excavation with rectangular cross-section in homogeneous massive rock mass of marble. The influence is demonstrated by the difference in stress distribution and plastic zone between two different simulation methods, one with constant viscous parameters and the other with varying viscous parameters. For all simulations, the model ran first to equilibrium to obtain the elastoplastic solution. Then the simulation activated the creep model to run for a predetermined time to obtain the viscoelastic-plastic solution. As the purpose of these simulations was to check the influence of the variation in the time-dependent property of rock before and after failure, size effect was not considered for the rock mass. In-situ stress and mining layout were two assumed causes for the failure around the underground excavation at equilibrium. As a result, the excavation in the assumed in-situ stress field could introduce a plastic zone in the surrounding rock mass. In the following creep simulation, one model changed the parameters for the failed zone while the other did not. The simulations assumed that the angle between the in-situ maximum horizontal stress and the longitudinal axis of an entry was $60^{\circ}$, which is an unfavorable condition for entry stability (Gadde and Peng 2004). For current modeling efforts the simulations considered only one in-situ stress condition, with the following conditions maintained as follows: vertical stress and minimum horizontal stress at 13.78 $\mathrm{MPa}$, maximum horizontal stress value at $22.39 \mathrm{MPa}$, and the ratio of maximum horizontal stress to the vertical stress or the ratio of maximum horizontal stress to minimum horizontal stress at 1.625 .

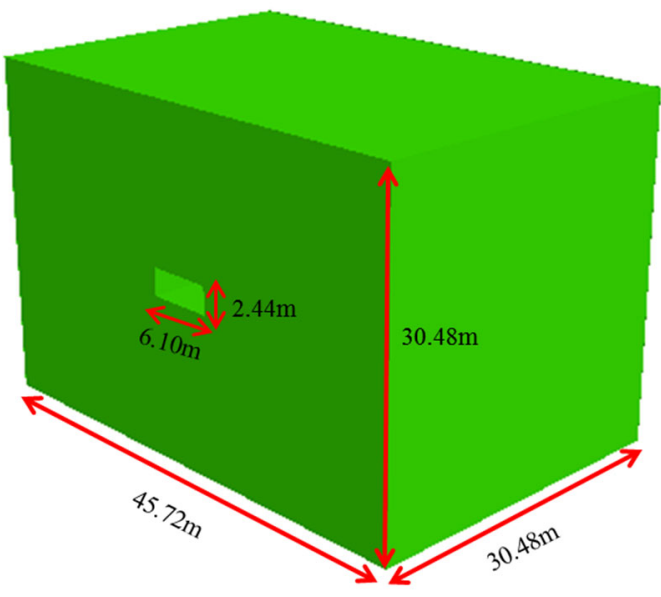

Fig. 4.8. Model dimension 


\subsubsection{Simulation results and discussion}

(1) Simulation results at equilibrium condition

To accurately simulate the geostatic stresses in the numerical simulation, the models ran to equilibrium conditions. Creep runs then activated from the equilibrium stage. The change in stress distribution drives the time-dependent deformation in the rock and the plastic state indicates the development of failure in the roof. Therefore, this investigates two parameters: (1) plastic state and (2) stress distribution at equilibrium condition. Figs. 4.9 and 4.10 present the major principal stress distribution and the plastic state at equilibrium. High stress concentration occurs at the corner of entry which allows for the onset of shear failure. In addition, the presence of directional in-situ horizontal stress increases the stress concentration at one corner, propagating failure towards the upper layers. The failure and stress distribution results are similar to the condition at the first stage of cutter failure process in underground coal mines (Gadde and Peng 2005).

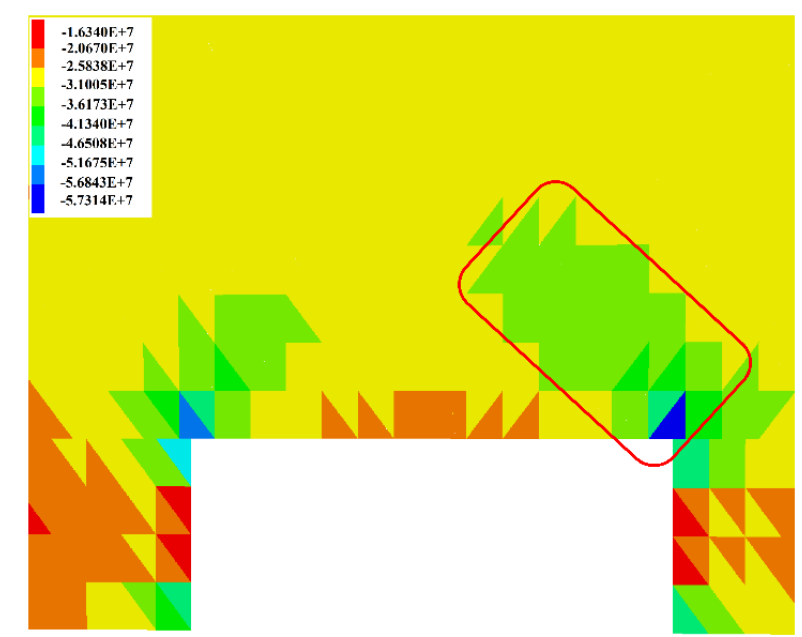

Fig. 4.9. Plot of major principal stress at equilibrium (unit: Pa)

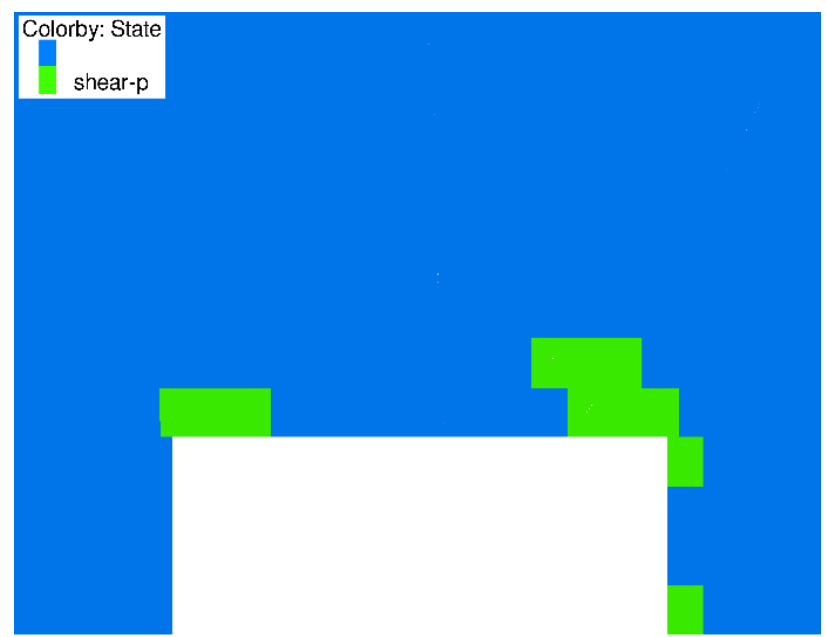

Fig. 4.10. Plot of plastic state at equilibrium 
(2) Simulation results with constant viscous parameters

The numerical model assigned with constant viscous parameters ran for time equivalent to one day. Figs. 4.11 and 4.12 plot the major principal stress distribution and failure condition, respectively. When Figs. 4.9 and 4.11 are compared, it is notable that the change in major principal stress distribution is insignificant and that more stress concentration still occurs at the right edge of the entry. The change from Fig. 4.9 to Fig.4.11 is the decrease in magnitude of the stress. In addition, when comparing Figs. 4.10 and 4.12, it shows that no failure propagation occurs during creep model run for one day. Therefore, the stress within the roof is relaxing at the same time and there is no failure propagation when constant viscous parameters for Burgers model are used.

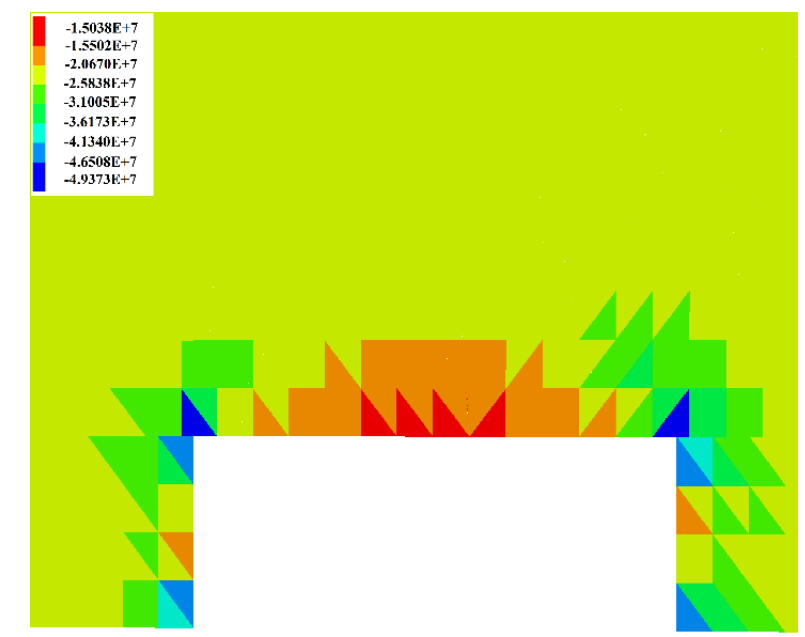

Fig. 4.11. Plot of major principal stress after one day for model with constant viscous parameters (unit: Pa)

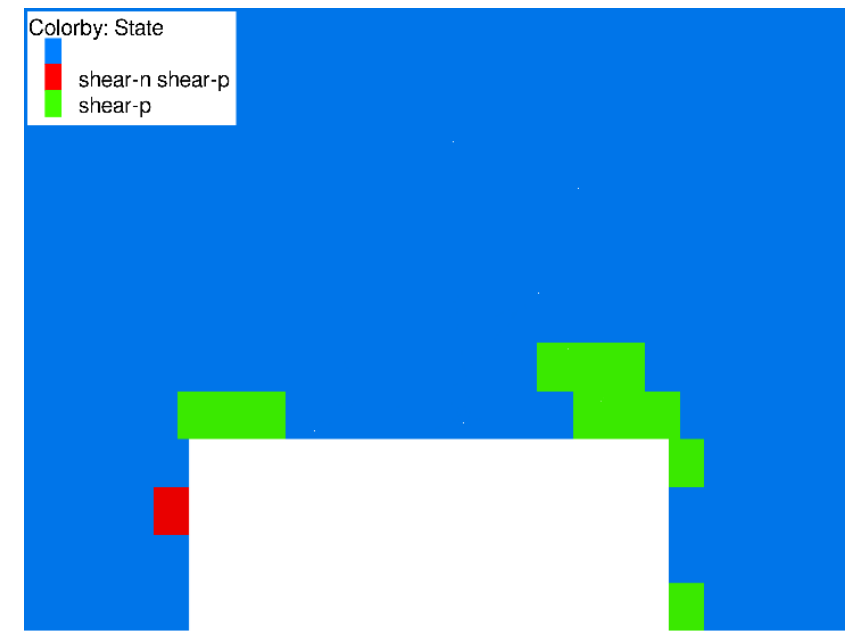

Fig. 4.12. Plot of plastic state after one day for model with constant viscous parameters

The observations show "synchronized" relaxation within the failed and elastic zones when using constant viscous parameters. There is no difference in the time-dependent parameters between intact rock and failed rock. If initiating from the same stress condition, the relaxation behavior of intact and failed rock is exactly the same. For the same time duration, equal amount of stress can relax from failed and intact rock, as shown in Fig. 3.13. Thus, there is no change in the stress distribution. However, due to stress relaxation, 
the magnitude of stress should decrease with time. This results in a kind of "synchronized" stress relaxation. In addition, due to "synchronized" stress relaxation, differential stress cannot build up and the reducing stress prevents failure propagation.

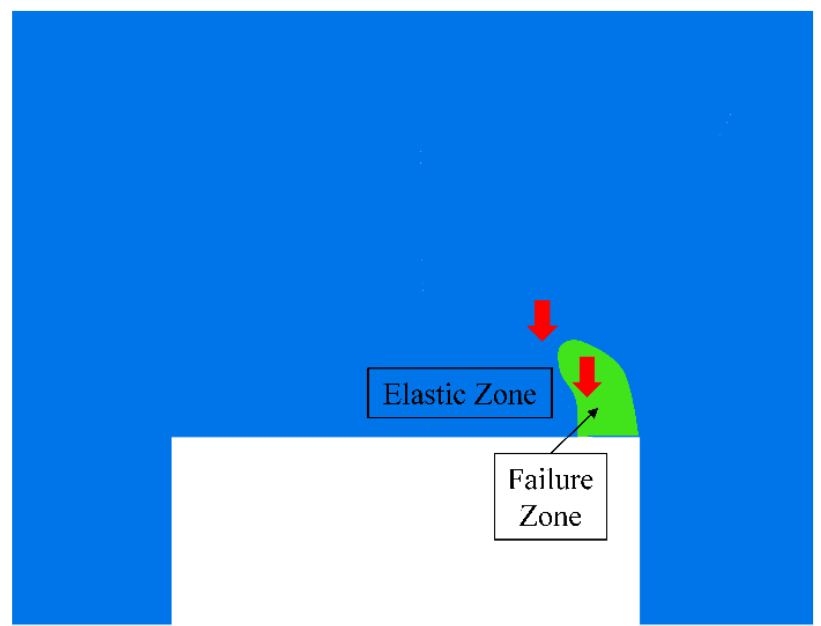

Fig. 4.13. Illustration of "synchronized" stress relaxation in plastic and elastic zone for the model with constant viscous parameters

(The size of the arrow represents the relative magnificence of stress relaxation)

(3) Simulation results with varying viscous parameters

With varying viscous parameters, the results predicted by the model significantly differ from the model with constant parameters. The model ran beyond equilibrium for one day. When Figs. 4.10 and 4.14 are compared, it is notable that failure initiates from the left and right edges of the entry; the failure initiating from the right edge propagates towards the upper strata. Also, the propagating direction and range of failure zone in Fig. 4.14 is similar to the distribution of high stress zone at equilibrium, as marked in Fig. 4.9. This demonstrates the importance of stress distribution on the propagation of failure with time.

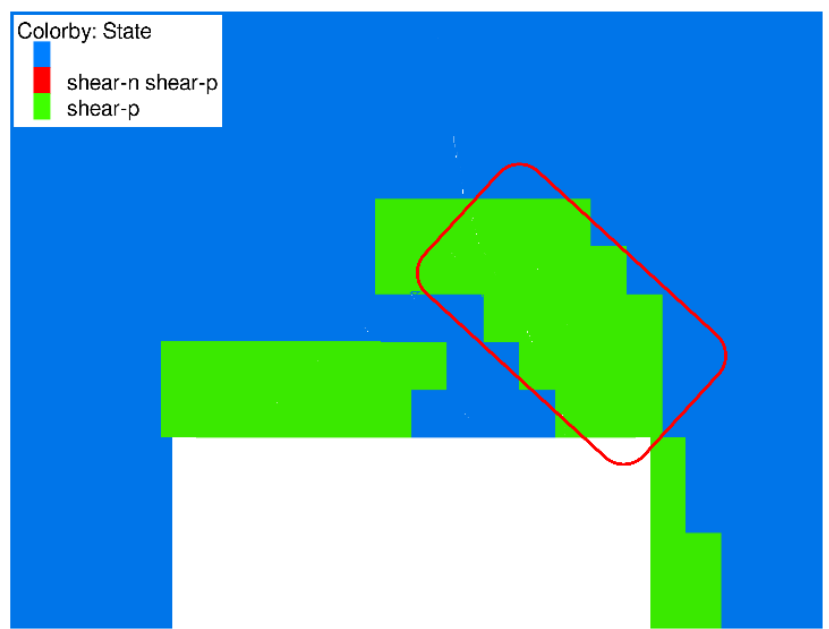

Fig. 4.14. Plot of plastic state after one day for model with varying viscous parameters

In addition, varying the viscous parameters when failure occurs affects the stress distribution. Fig. 4.9 shows that stress concentrations are observed above the opening corners. However, in Fig. 4.15 the stress 
within these corresponding areas becomes very small after creep simulation of one day. Comparing Figs. $4.9,4.14$, and 4.15 shows that after creep simulation of one day, the high stress areas at equilibrium (Fig. 4.9) already fail (Fig. 4.14), and the stress within these areas become very small (Fig. 4.15). Areas with high stress move deeper into the roof and surround the areas with low stress. These results demonstrate that areas with high stress at equilibrium gradually fail with time, and the stress transfers from the failure zone into deeper solid rock.

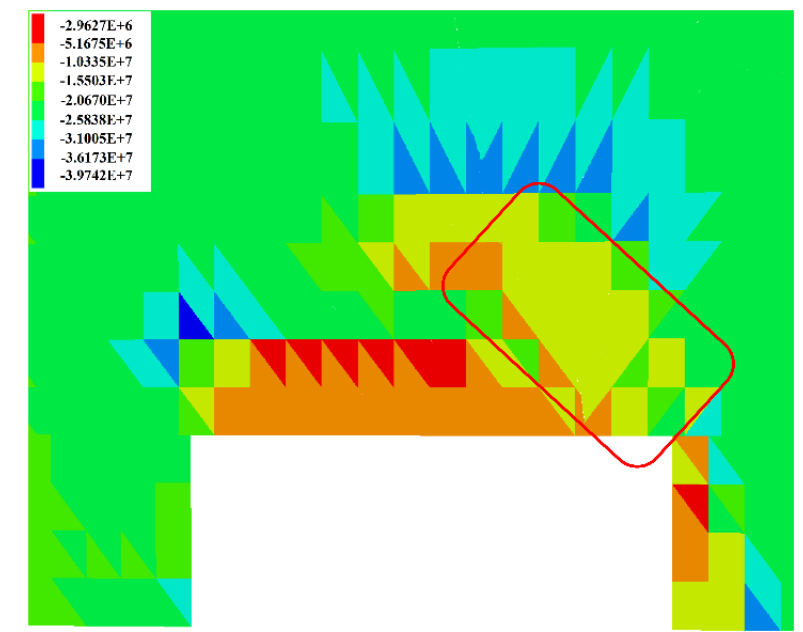

Fig. 4.15. Plot of major principal stress after one day for model with varying viscous parameters (unit: Pa)

The observations demonstrate that varying the time-dependent parameters can result in different relaxation behaviors. Due to the decrease in the time-dependent parameters, failed rock can relax more stress than intact rock from the same stress level, as shown in Fig. 4.16. Pronounced stress relaxation in the failure zone increases differential stress in surrounding elastic zones and, in turn, increases the failure potential of the surrounding elastic zones.

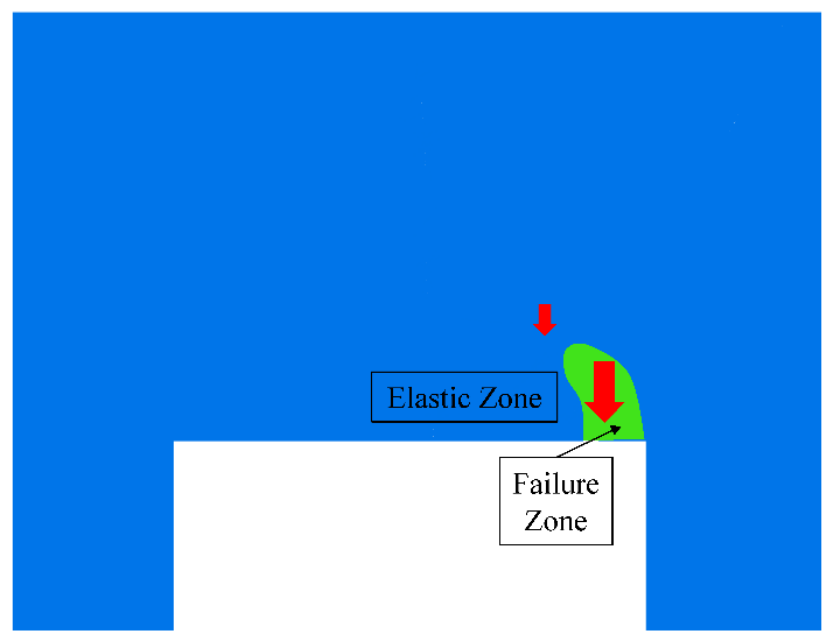

Fig. 4.16. Illustration of stress relaxation in plastic and elastic zone for the model with varying viscous parameters (The size of the arrow represents the relative magnificence of stress relaxation)

(4) Mechanism for time-dependent propagation of failure

The mechanism for time-dependent propagation of failure is summarized based on the numerical model. 
Fig.4.17 illustrates the failure process. The stress redistribution caused by excavation initiates shear failure within roof, especially at the entry corners. Difference in the time-dependent property causes the failed rock to relax the stress at a faster rate than that of the intact rock. The rock surrounding the broken rock is intact and therefore still elastic in response. However, rapid stress relaxation in the adjacent failure zone decreases confinement to the surrounding rock and, in turn, increases differential stress, especially at the boundary between the failure and elastic zones. If the time-dependent differential stress increases to meet the rock strength, failure can occur at the boundary of failure zone and result in the time-dependent failure propagation.

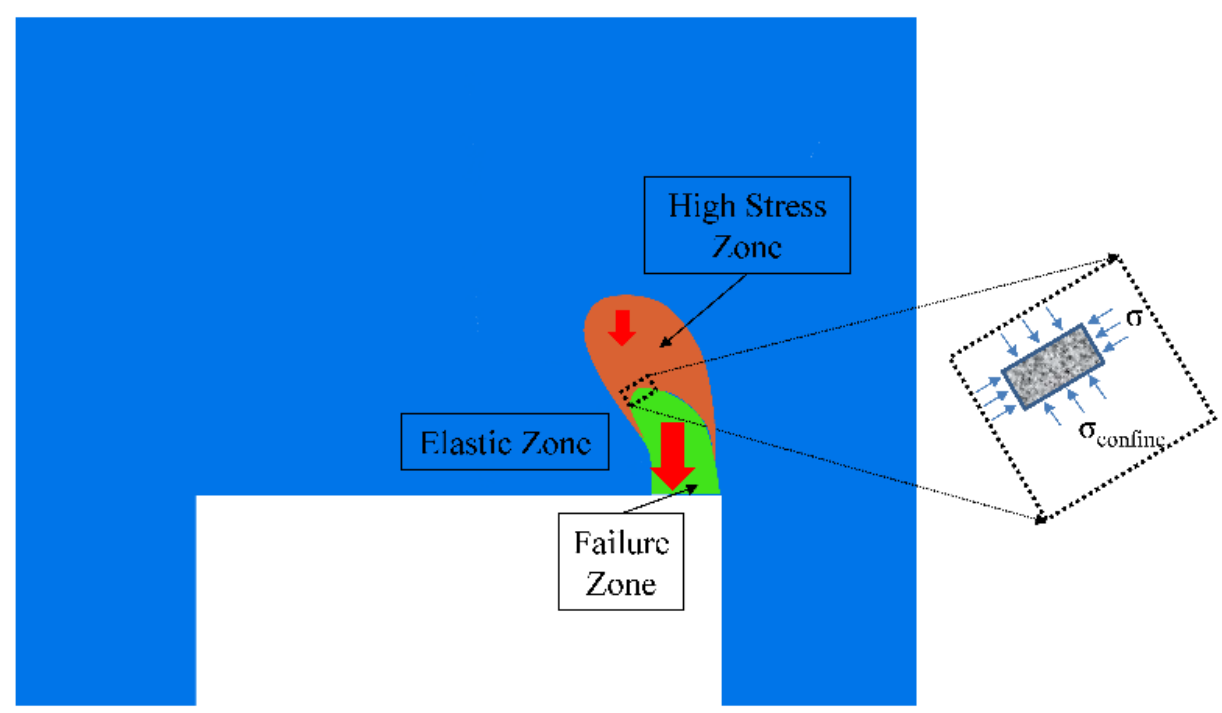

Fig. 4.17. Illustration of mechanism for time-dependent propagation of failure

However, the modeling results demonstrate that the time-dependent failure process is dependent on various factors. First, the difference in the time-dependent properties between intact rock and failed rock can affect the failure process. When using constant viscous parameters, there was no failure propagation in the numerical simulation. However, failure propagated when using varying viscous parameters for intact and failed rock. The mechanism for time-dependent failure propagation demonstrates that time-dependent failure induces due to the increase of differential stress resulting from different stress relaxation rates within the failure and elastic zone. If the variation in the time-dependent properties decreases, the difference in stress relaxation within the failure zone and elastic zones decreases and, results in "synchronized" stress relaxation.

Second, the failure zone at equilibrium determines the initial condition for the time-dependent failure. The failure zone at the entry corners initiates due to the stress redistribution during entry development. The modeling results show that high differential stress builds up at the boundary between the failure zone and elastic zone. Therefore, time-dependent failure starts to propagate from the boundary of the failure zone. Due to the bedded rock strata and/or in-situ horizontal stress in underground coal mines, failure around an entry is usually localized. This location is very important for the time-dependent failure propagation, as it determines where the time-dependent failure begins. If no failure zone forms during entry development, the rocks within the roof are elastic with same time-dependent parameters and, in turn, "synchronized" stress relaxation occurs. 
Finally, failure propagation depends on in-situ stress. On one hand, in-situ stress can affect the formation of a failure zone during entry development; as such, it can affect the time-dependent failure process. If the panel direction varies, in-situ horizontal stress can affect the size and location of the failure zone. On the other hand, in-situ stress controls the time-dependent failure propagation. In-situ stress determines stress distribution around an opening; this in turn determines the high stress zone. When differential stress is building up due to stress relaxation within the failure zone, differential stress in a high-stress zone has a higher chance to meet the rock strength and induce failure than that with low stress. Thus, failure is prone to propagate along the direction of high major principal stress. In conclusion, in-situ stress determines the direction of time-dependent failure propagation.

(5) Influence of failure propagation on roof convergence

This study investigated the influence of failure propagation on roof convergence by comparing the roof convergence for the models with constant and varying parameters and then observing the different roof convergence behaviors. As shown in Fig. 4.18, when starting from the same equilibrium condition, the difference in the two roof convergence curves becomes larger and larger with time. This is the result of time-dependent failure propagation. Fig. 4.18 also presents the plastic state at different times for the model with varying parameters. After the primary stage, the convergence curve with varying parameters becomes steeper and steeper along with the gradual failure propagation; the convergence curve with constant viscous parameters, conversely, shows almost constant convergence rate with small fluctuation in the secondary stage.

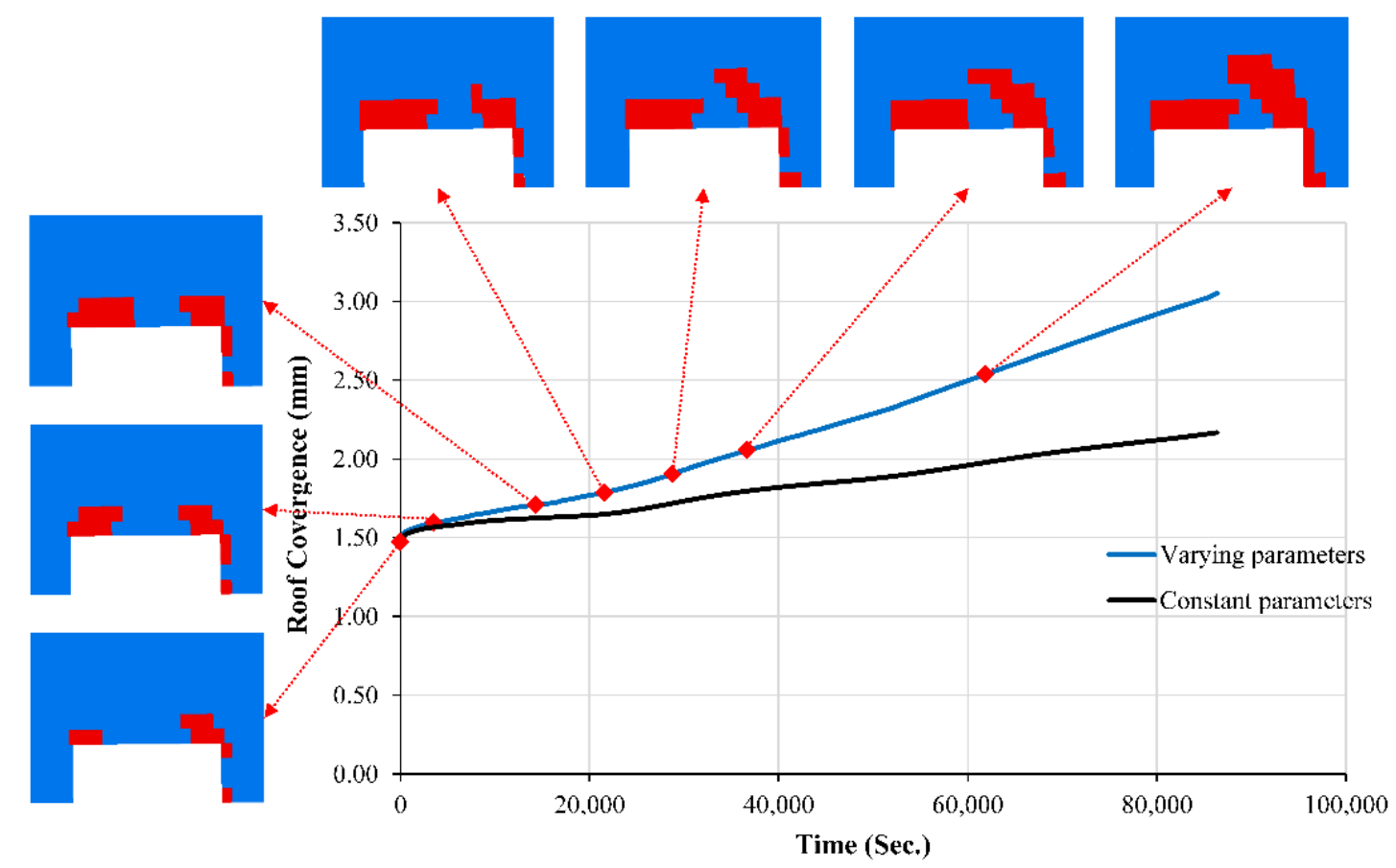

Fig. 4.18. Comparison of roof convergence for the models with constant and varying parameters 


\subsection{INFLUENCE OF STRENGTH DEGRADATION ON TIME-DEPENDENT ROOF FAILURE}

Failure of rock specimen in creep tests are known to occur at stresses that are lower than the strength of the intact rock (Wawersik and Brown 1971; Baud and Meredith 1997; Rinne 2008), indicating that the rock strength degrades during the creep test. The acoustic emission output from triaxial creep test on sandstone shows that time-dependent cracking plays a major role during the secondary stage of creep (Baud and Meredith 1997). Rinne (2008) adopted subcritical crack theory to simulate the delayed failure of a loaded rock, with the result demonstrating that the slow time-dependent fracturing process may lead to a sudden unstable failure event. Lin et al. (2009) carried out a series of constant loading tests with acoustic emission monitoring; the source location analysis reproduced the progressive damage process during the test. These studies demonstrate that subcritical cracks can propagate during creep test and therefore deteriorate the rock specimens. If the constitutive mode and numerical simulations do not consider the strength degradation caused by viscous deformation, the strength remains constant during creep test. The applied stress is constant and is lower than the rock strength; therefore, the specimen deforms without failure.

This is the situation for the built-in constitutive model in Itasca software. The constitutive models for the time-dependent simulations are the combination of creep models and Mohr-Coulomb failure criterion. The constitutive models do not include strength degradation. Rock failure occurs only when the stress condition meets the failure criterion. The viscoelastic-plastic model, combining Burgers model and MohrCoulomb failure criterion, is the cvisous model in 3DEC (Itasca 2012), which was used to simulate an unconfined creep test. The simulation applied a constant stress of ninety-eight percent of the strength onto the top of the simulated specimen. The model ran to equilibrium condition first to obtain the elastic solution. The instantaneous elastic strain was $4.35 \times 10^{-4}$. Creep simulation then activated to run for a time equivalent to $2 \times 10^{6}$ seconds (555.6 hours). As shown in Fig. 4.19, the specimen deforms without failure with the axial viscous strain is almost seventeen times as the instantaneous elastic strain. However, this is not the situation in laboratory tests.
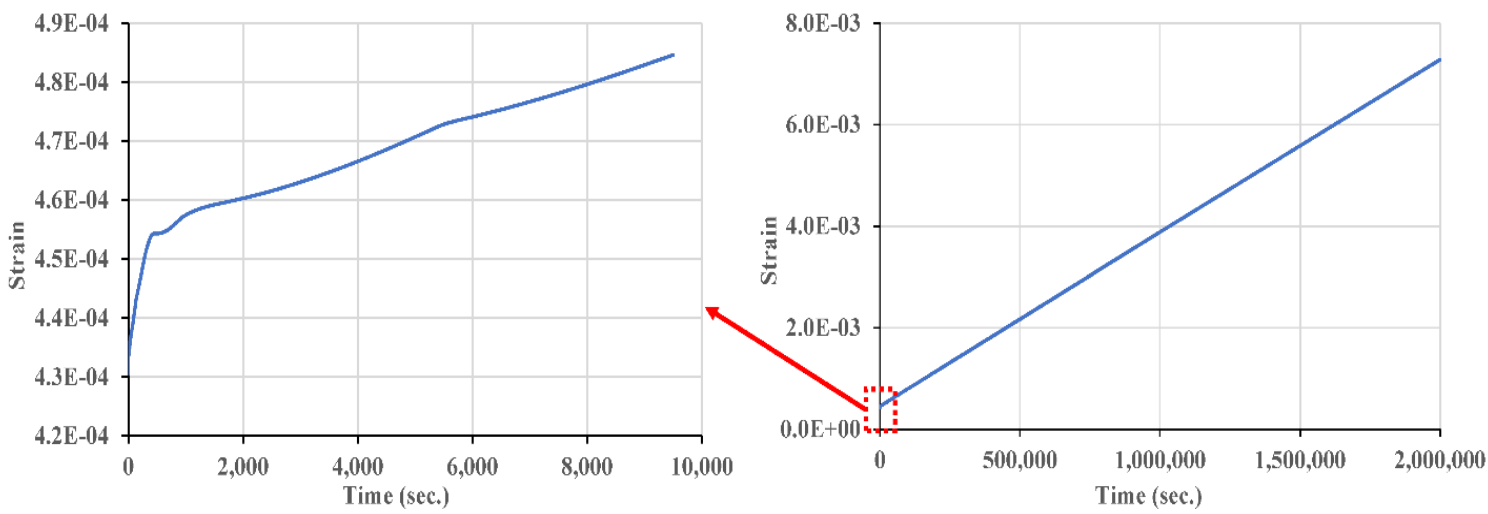

Fig. 4.19. Stress-strain curve for the simulated unconfined creep test with Burgers model and Mohr-Coulomb criterion

In order to capture the failure under creep condition, studies should consider the strength degradation induced by viscous deformation. Various studies have proposed methods to consider the strength degradation induced by viscous deformation. Wawersik and Brown (1971) used maximum creep strain as 
failure criterion, which is obtainable from the strain between the ascending and descending parts of the complete quasi-static stress-strain curve. The results of uniaxial compression creep tests (Kranz and Scholz 1977) showed that the inelastic volumetric strain at the onset of tertiary creep was nearly constant, independent of stress level and related to fracture strength and rock type. Malan (1999) also derived a timedependent Mohr-Coulomb failure criterion, where the cohesion was considered to degrade with time and the degradation rate depended on the distance between the stress state and the residual strength. Fakhimi (1994) proposed a model to investigate the stand-up time of underground excavations where the strength degraded exponentially with effective viscous strain. Similar to the effective shear plastic strain in strainsoftening model, the effective viscous strain is a parameter qualifying the damage induced by the viscous behavior and can be related to the rock strength. With such a parameter, the strength degradation caused by viscous deformation can be considered for complicated stress condition and be used in numerical simulation. This section used the concept of effective viscous strain to quantify the damage induced by viscous strain and to control the strength degradation due to viscous strain with FISH functions.

\subsubsection{Effective viscous strain for Burgers materials}

Burgers model has two components: one for the time-independent response, and the other for the timedependent behavior, as shown in Eq. 4.1. The relation between the viscous strain and time in axial direction can then be described as follows:

$$
\varepsilon^{v}=\frac{\sigma}{3 \eta_{m}} t+\frac{\sigma}{3 E_{k}}\left[1-\exp \left(\frac{-E_{k} t}{\eta_{k}}\right)\right]
$$

Introducing the strength degradation parameter $\kappa^{v}$ controls the time-dependent strength degradation. It is a measure of the second invariant of the viscous strain, given as,

$$
\kappa^{v}=\sqrt{\frac{2}{3} \varepsilon_{i j}^{v} \varepsilon_{i j}^{v}}
$$

where $\varepsilon_{i j}^{v}$ represents the viscous strain in three-dimensional condition. For unconfined condition, one can then derive the strength degradation as,

$$
\kappa^{v}=\frac{\sqrt{3}}{2}\left|\varepsilon^{v}\right|
$$

The strength degradation parameters can be calculated with the axial strain during an unconfined creep test. At the same time, determining the relation between $\kappa^{v}$ and strength value is possible if the unconfined creep test continues until the specimen fails. Under this circumstance, the constant stress applied to the specimen is the strength value. Due to the viscous deformation, the rock strength decreases from the original value to the stress value of the creep test. Therefore, this obtains the strength value for one specimen with specific effective viscous strain values.

After obtaining the relation between the strength degradation parameters and strength value from creep tests, it is then enterable into 3DEC code to control the strength degradation during time-dependent simulations. At each timestep, one can calculate the accumulated effective viscous strain and then use it to update the strength value based on the strength degradation relation. The calculation of effective viscous strain in $3 \mathrm{DEC}$ is as follows. 


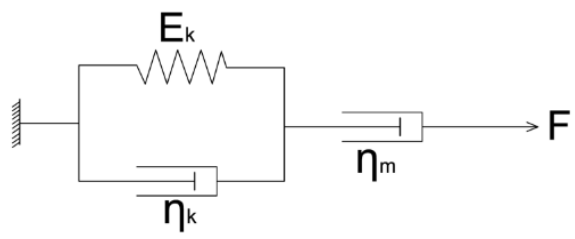

Fig. 4.20. Schematic of viscous part in Burgers model for one dimension

Fig. 4.20 only considers the viscous part of Burgers model. For the Maxwell unit, there is,

$$
\dot{u}_{m}=\frac{\bar{F}}{\eta_{m}}
$$

where $\dot{u}_{m}$ is the strain rate of the Maxwell unit and $\bar{F}$ corresponds to the mean values of $F$ over the timestep with the format of Eq. 4.5. The superscripts ${ }^{\mathrm{n}}$ and ${ }^{\mathrm{o}}$ denote the new and old values, respectively.

$$
\bar{F}=\frac{F^{n}+F^{0}}{2}
$$

Writing Eq. 4.4 in finite-difference form,

$$
u_{m}^{n}=u_{m}^{0}+\frac{F^{n}+F^{0}}{2 \eta_{m}} \Delta t
$$

For the Kelvin part (Itasca 2012), there is,

$$
u_{k}^{n}=\frac{1}{A}\left[B u_{k}^{0}+\left(F^{n}+F^{0}\right) \frac{\Delta t}{2 \eta_{k}}\right]
$$

where

$$
\begin{aligned}
& A=1+\frac{E_{m} \Delta t}{2 \eta_{k}} \\
& B=1-\frac{E_{m} \Delta t}{2 \eta_{k}}
\end{aligned}
$$

At each timestep, one can calculate the new viscous strain by adding up the Maxwell viscous strain in Eq. 4.6 and the Kelvin strain in Eq. 4.7. For three-dimensional condition, the symbols $S_{i j}$ represents the deviatoric stress tensor and $\varepsilon_{i j}^{v}$ represents the viscous strain tensor. The superscripts $k, m$, and $p$ represent the contributions of the Kelvin, Maxwell, and plastic components of stresses and strains, respectively.

$$
\begin{gathered}
e_{i j}^{m, n}=e_{i j}^{m, o}+\frac{\Delta t}{4 \eta^{m}}\left(S_{i j}^{n}+S_{i j}^{o}\right) \\
e_{i j}^{k, n}=\frac{1}{A}\left[B e_{i j}^{k, o}+\frac{\Delta t}{4 \eta^{k}}\left(S_{i j}^{n}+S_{i j}^{o}\right)\right]
\end{gathered}
$$

Further calculating the viscous strain tensor as,

$$
e_{i j}^{v}=e_{i j}^{m}+e_{i j}^{k}
$$

then substituting this into Eq. 4.2 to calculate the accumulated effective viscous strain at each timestep. 


\subsubsection{Incorporation of strength degradation into numerical simulation}

Laboratory creep tests can determine the strength degradation relation. Unconfined creep tests can run at different stress levels until the specimens fail. For each stress level, Eq. 4.3 can calculate the accumulated effective viscous strain. As the specimens fail at the end of the creep tests, the applied stress values are the rock strengths after degradation. By determining and combining the degraded strength and accumulated effective viscous strain for each stress level, one can find the strength degradation relation.

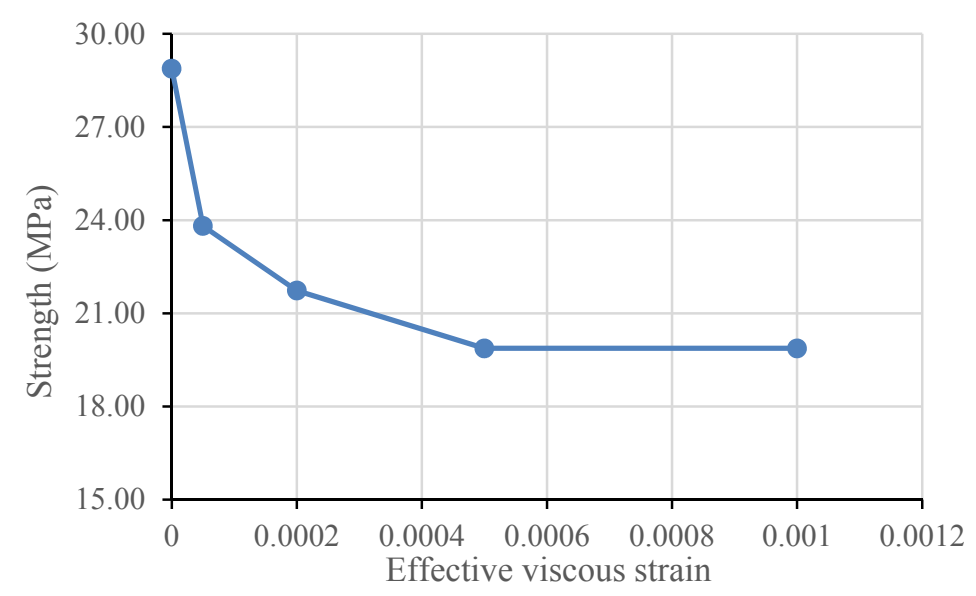

Fig. 4.21. Strength degradation relation

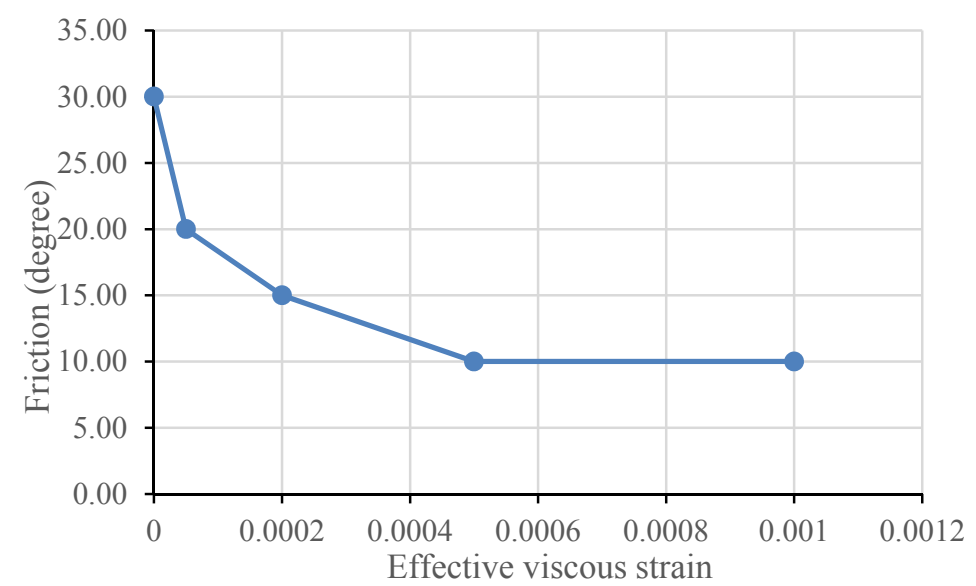

Fig. 4.22. Relation between friction angle and effective viscous strain used in the simulation

A FISH function was written to control the strength degradation during the time-dependent simulations. The cvis model in 3DEC, Burgers model for the time-dependent behavior and Mohr-Coulomb criterion for failure prediction, represented the time-dependent behavior of rock. At each timestep, the FISH function calculated the accumulated effective viscous strain and updated the strength values. Here the study assumed the cohesion value to be constant and the friction angle decreased with accumulated viscous strain to account for the time-dependent strength degradation. The relation between strength value and effective viscous strain was converted to the relation between friction angle and effective viscous strain based on a constant cohesion value, as shown in Figs. 4.21 and 4.22. At each timestep, Eqs. 4.2 and 4.12 calculated the accumulated effective viscous strain and updated the corresponding friction angle. With the increase in effective viscous strain, friction angle gradually decreased resulting in strength degradation. 


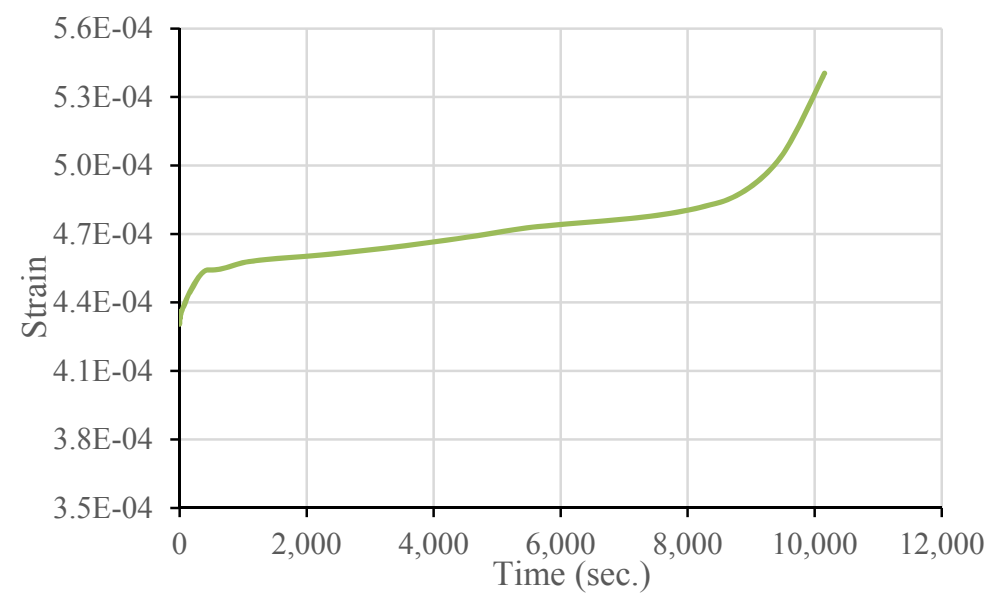

Fig. 4.23. Creep curve showing three stages for the specimen considering strength degradation

One unconfined creep test was simulated to verify the simulation method. Fig. 4.22 shows the strength degradation relation represented by the relation between friction angle and the effective viscous strain. The effective viscous strain gradually accumulates with time during a creep test, leading to the decreasing strength value. Specimen failure occurs when the strength value reduces to the value equal to the applied constant stress. Fig. 4.23 illustrates that the simulated specimen demonstrated typical creep behavior where the creep curves had three stages-primary, secondary, and tertiary. The accelerating strain rate after the secondary stage indicated the failure of the specimen. Therefore, the simulated specimens failed after some time when the creep test initiated.

\subsubsection{Numerical study on the influence of strength degradation on roof behavior}

This study examined the influence of strength degradation on roof behavior within 3DEC with the FISH function controlling the strength degradation during the time-dependent simulations. Table 4.2 and Fig. 4.22 present the parameters and Fig. 4.8 shows the model used for the simulations. For one model, constant viscous parameters accounted for the influence of the strength degradation, while the other model used varying viscous parameters to account for the influence of strength degradation and the variation in the time-dependent property.

As Burgers model describes elastic behavior under hydrostatic condition and describes Burgers behavior under deviatoric stress condition, the time-dependent behavior only occurs with deviatoric stress and the distribution of deviatoric stress potentially affects the time-dependent behaviors of roof. Fig. 4.24 plots the distribution of deviatoric stress. An arch-shaped area with high deviatoric stress exists within the roof. In addition, Fig. 4.25 shows the distribution of maximum shear stress. The maximum shear stress represents the difference between major principal stress and minor principal stress, on which the Mohr-Coulomb failure criterion is based. The maximum shear stress shows similar distribution as the deviatoric stress, indicating that the arch-shaped area has high deviatoric stress and high maximum shear stress. The high deviatoric stress implies that both large time-dependent deformation and strength degradation will manifest. The high maximum shear stress indicates that the area is closer to the peak strength than other areas. If there is time-dependent failure propagation, it will likely propagate along the arch-shaped area. 


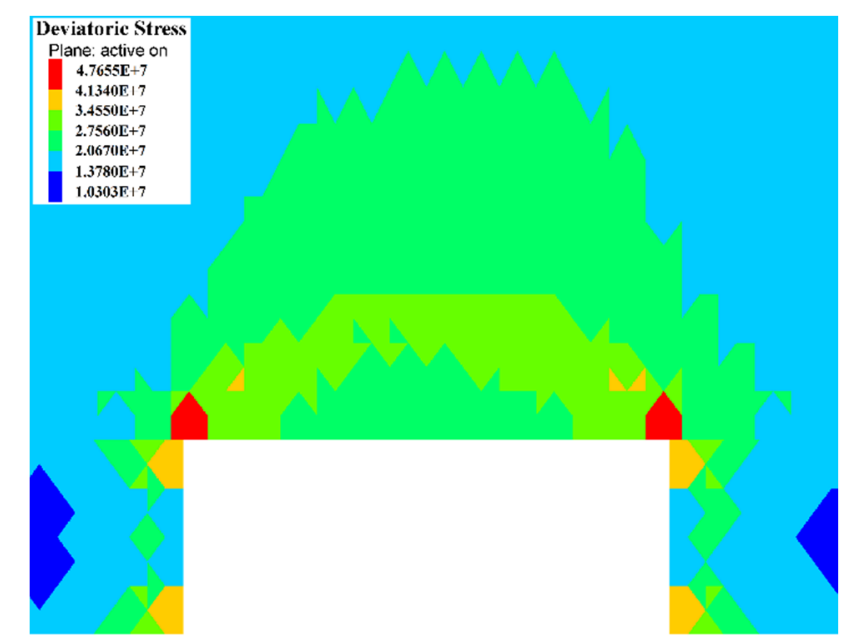

Fig. 4.24. Plot of deviatoric stress distribution at equilibrium (Unit: Pa)

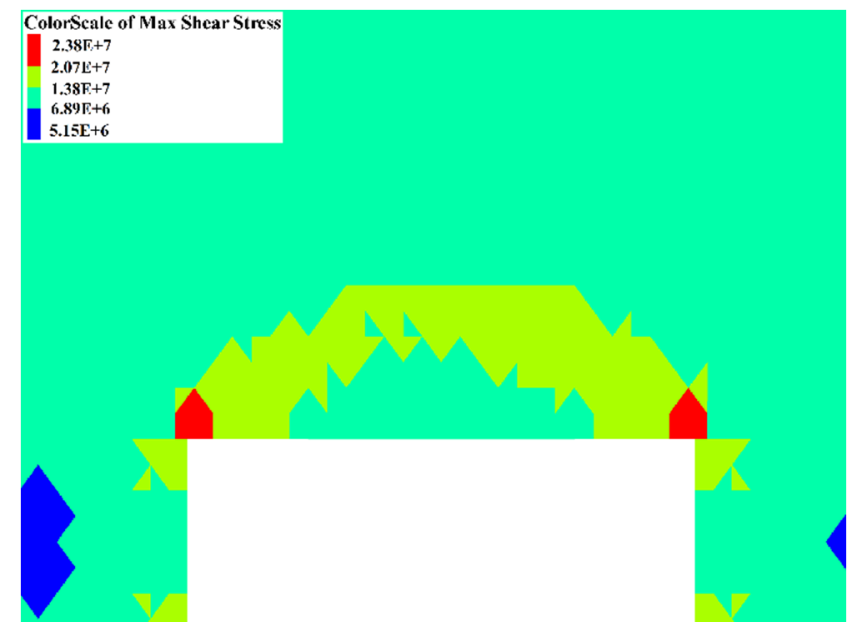

Fig. 4.25. Plot of maximum shear stress distribution at equilibrium (Unit: Pa)

In addition, the time-dependent simulation activated and ran for one day after reaching the equilibrium condition. Figs. 4.26, 4.27 and 4.28 show the failure condition, effective viscous strain distribution and friction distribution within the roof, respectively. The comparison of failure condition in Figs. 4.10 and 4.26 shows that there was minor change in the failure condition, where slight failure propagation occurred above the left entry corner. As the simulation used constant viscous parameters, the stress in the failure zone and the surrounding elastic zone relaxed with the same viscous parameters. Strength gradually degraded with accumulated viscous strain, especially within the arch-shaped area, as shown in Figs. 27 and 28. However, the stress relaxed from the rocks along with the viscous deformation, meaning that the strength of the roof rock reduced with viscous deformation at the same time as the stress of the rocks decreased. The result was no failure propagation with time. Furthermore, the model with constant viscous parameters ran for thirty more days, and the results show that there was no failure propagation over one month with these simulation conditions. 


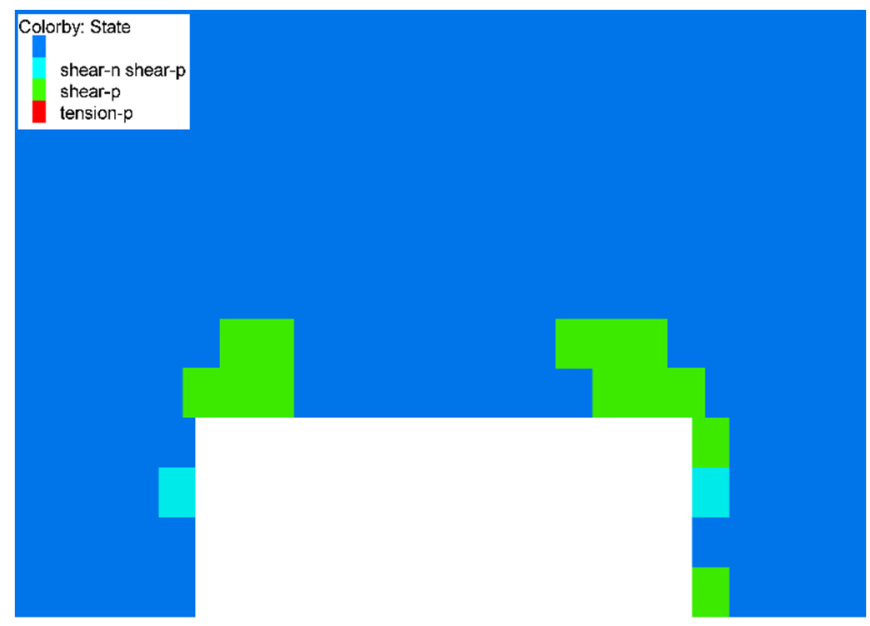

Fig. 4.26. Plot of plastic state after one day for model with constant viscous parameters and strength degradation

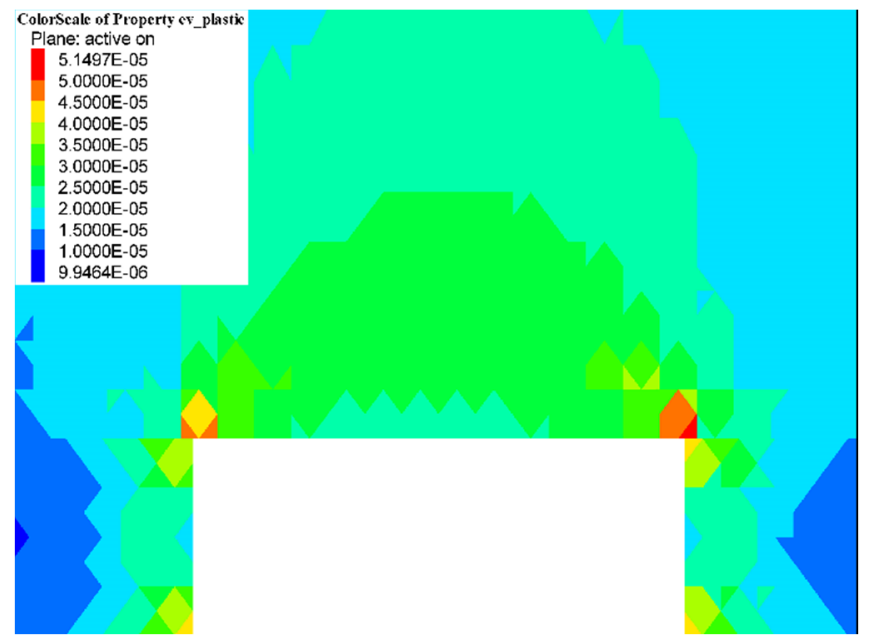

Fig. 4.27. Plot of effective viscous strain distribution after one day for the model with constant viscous parameters and strength degradation

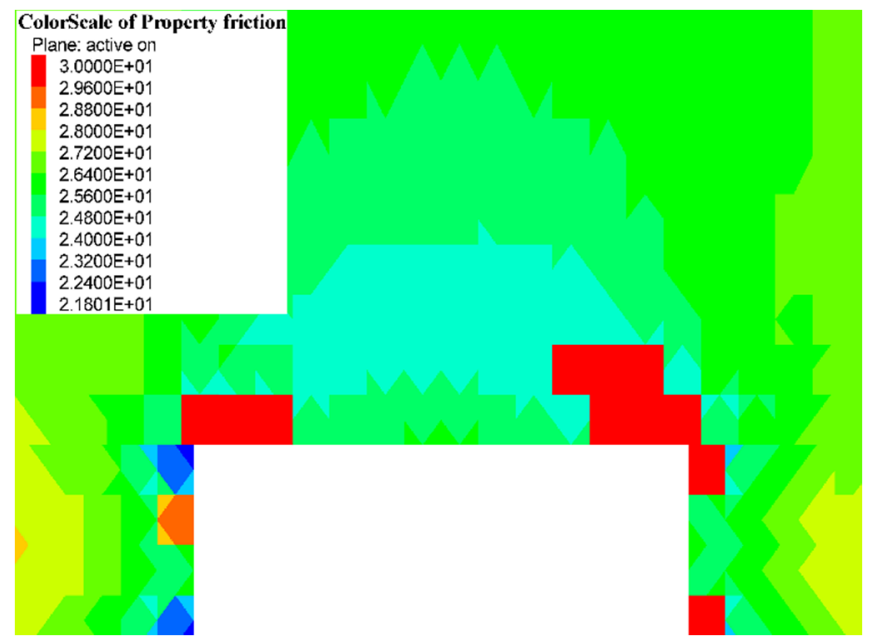

Fig. 4.28. Plot of friction angle after one day for the model with constant viscous parameters and strength degradation (Unit: degree) 
Finally, for the model with varying viscous parameters, Figs. 4.29 and 4.30 show the plastic states after two minutes and after one day, respectively. Firstly, this study examined the influence of varying viscous parameters for the models with strength degradation. Comparing Figs. 4.24, 4.25, and 4.29 shows that the failures initiating from the entry corners at equilibrium propagated quickly along the high deviatoric stress and high maximum shear stress area and coalesced in around two minutes. The failure zone gradually propagated above, leading to a much larger failure zone than the model with constant viscous parameters. The difference between these two models was the viscous parameters in the post-failure region. Due to the difference in the viscous parameters between intact and failed rock, the stress relaxation rates were different inside and outside the failure zone. The higher stress relaxation rate inside the failure zone increased the stress difference at the boundary between failure and elastic zones, potentially promoting the failure propagation. The increasing stress difference also accelerated the strength degradation and thus promoted the failure propagation.

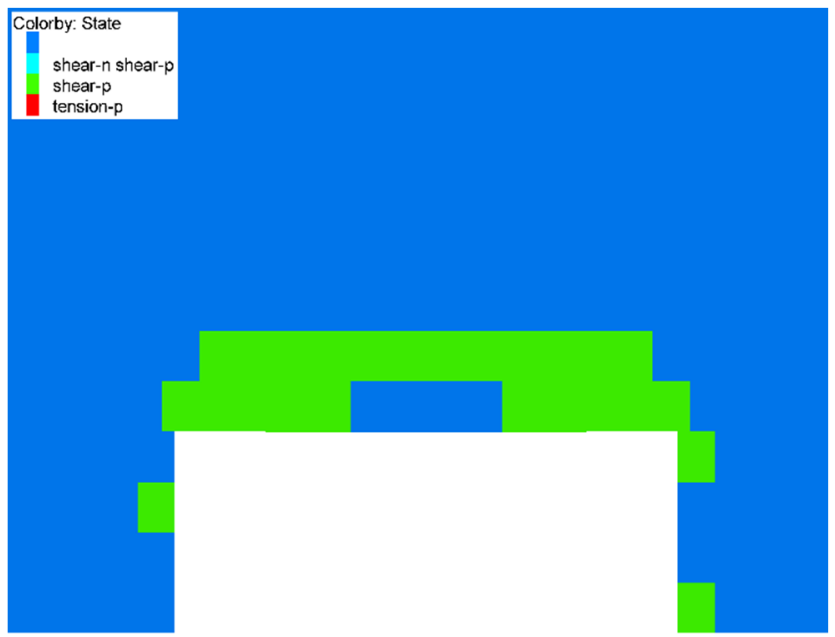

Fig. 4.29. Plot of plastic state after two minutes for the model with varying parameters and strength degradation

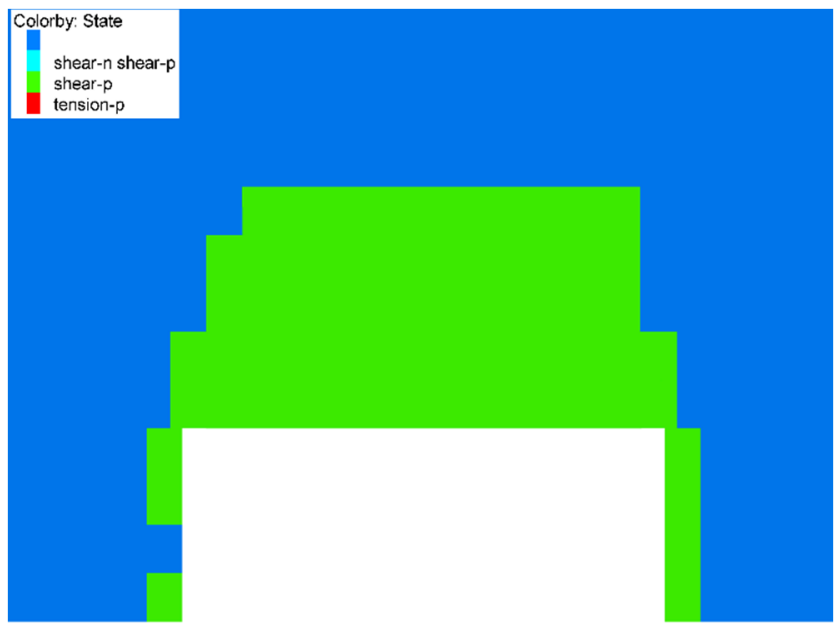

Fig. 4.30. Plot of plastic state after one day for model with varying parameters and strength degradation

Additionally, this study examined the influence of strength degradation for the models with varying viscous parameters. The difference in the plastic states showing in Figs. 4.14 and 4.30 is a result of the 
strength degradation. While the model in Fig. 4.30 considered the influence of strength degradation, the model in Fig. 4.14 did not. In Fig. 4.14, the failure initiating from the two entry corners propagated separately towards the upper strata. When the strength degradation was taken into account, the plastic states shown in Figs. 4.29 and 4.30 demonstrate that the failure propagated to coalesce first and then propagated upwards, resulting in a larger failure zone. Therefore, the consideration of strength degradation changed the failure process and increased the failure zone.

\subsection{SUMMARY}

The formation of roof fall is a time-dependent process. The time-dependent behavior of roof rocks is the key to determine the time-dependent response of mine roof. However, for time-dependent numerical simulations, commercial codes do not consider the variation in the time-dependent property and strength degradation caused by viscous behavior. This chapter investigated the influence of these two behaviors on the time-dependent response of mine roof by using FISH functions to change the viscous parameters when failure occurred and to control the friction decay with effective viscous strain in 3DEC. The model results demonstrate the importance of these two factors on the time-dependent deformation and failure of mine roof.

The relaxation test was used as the test method to study the time-dependent property of rock. Chapter 3 verified the method of using relaxation test to determine the viscous parameters in the pre-failure region. By assuming viscoelastic behavior for both intact rock and failed rock, Burgers model can describe the varying time-dependent behavior of rock before and after failure, and the method can determine the viscous parameters in the post-failure region. Varying viscous parameter can represent the variation in the timedependent property. Based on the derived relaxation equation and curve-fitting procedure, the viscous parameters for Burgers model in the pre-failure and post-failure region were determined from previous unconfined compression tests with various relaxation tests. The results demonstrate that there is pronounced difference in the time-dependent parameters between intact and failed rock, while the variation at different stages in the post-failure region is negligible. Therefore, it is rational to use two groups of time-dependent parameters to represent the time-dependent property of intact and failed rock.

In addition, the simulation, with constant and varying viscous parameters, demonstrates that the variation in the time-dependent property of rock can induce time-dependent failure propagation depending on various factors. Rapid stress relaxation in a failure zone decreases the confinement to the surrounding intact rock and increases the differential stress, potentially inducing failure at the boundary of the failure zone. This is the mechanism for the time-dependent failure propagation due to the variation in the timedependent property. However, this time-dependent failure process depends on various factors, like the failure zone at equilibrium, in-situ stress, and the difference in the time-dependent property for intact and failed rock. Furthermore, along with the time-dependent failure, increasing roof convergence rates can also occur.

Another important feature of the time-dependent behaviors of rocks is the strength degradation with time. Failure of rock specimen in creep tests are known to occur at stresses that are lower than the strength of the intact rock. Due to subcritical crack propagation and other mechanisms, the viscous deformation 
introduces damage to the rock specimens and therefore induces strength degradation. However, the viscoelastic-plastic model combining the viscoelastic model and the failure criterion cannot reproduce the creep failure observed in laboratory tests. This study introduced the parameter of effective viscous strain to quantify the damage induced by viscous deformation and to control the strength degradation in numerical simulation with 3DEC. Laboratory creep test can then determine the relation between strength values and the effective viscous strains. During numerical simulation, the strength value can be updated at each timestep after calculating the accumulated effective viscous strain. The simulation of rock specimen failure undergoing unconfined creep test demonstrates the ability of the FISH function in capturing the timedependent strength degradation. In the following simulations of mine roof, there was no failure propagation with constant viscous parameters for the simulated conditions and parameters. Although strength degradation occurred within the mine roof, the stress relaxed from the rocks at the same time; therefore, there was no time-dependent failure. However, a much larger failure zone occurred when varying viscous parameters were used. The increase in the stress difference at the boundary between elastic and failure zone resulted from the variation in the time-dependent property accelerates the strength degradation, and potentially promote the failure propagation. Finally, the consideration of strength degradation for the model with varying viscous parameters changes the failure process. For the model with varying viscous parameters, the failures initiating from the two entry corners propagate upwards separately, while the failures initiating at two different locations coalesce first and then propagate upwards when strength degradation is taken into account. 


\section{CHAPTER 5 \\ LABORATORY STUDY ON THE TIME-DEPENDENT BEHAVIOR OF INTACT AND FAILED ROCKS WITH UNCONFINED RELAXATION TEST}

\subsection{INTRODUCTION}

Fig. 1.3 presents the development of cutter type failure in coal measure rocks overlying the Pittsburgh coal seam. Failure usually initiates on one side of the entry during development. The rock within this failed zone is in the post-failure region and loses some or all of its load-bearing capacity. This loss alters the stress field and deformability of the roof. As a result, failure can progressively propagate and eventually leads to roof collapse. Simulations have used the strain-softening model to show the gradual loss of load-bearing capacity in the fractured zone during mine development (Gadde and Peng 2005; Ray 2009). However, the cutter failure in underground mines gradually develops with time. As shown in Fig. 1.3, intact rock, failed rock, and time-dependent failure propagation are all important in this process. In addition, the numerical simulations performed by Malan et al. (1997) demonstrate the importance of the time-dependent behavior of failed rock and the failure process in the time-dependent closure of underground excavations. Chapter 4 also investigates the influence of the variation in the time-dependent property on roof failure. The simulation results demonstrate that the difference in the time-dependent property between intact and failed rock leads to different stress relaxation rates inside and outside the failure zone; this variation results in the increase of stress difference at the boundary between failure zone and elastic zone, as well as potentially promoting the failure propagation. Therefore, it is necessary to determine the time-dependent property of intact and failed coal measure rocks, which can numerically simulate the time-dependent roof failure process in underground coal mines. The purpose of this chapter is to study the time-dependent behavior of intact and failed coal measure rocks.

Relaxation test has been proposed and verified as an alternative method to determine the time-dependent property of rocks, while creep tests commonly investigate the time-dependent behavior of intact rock. There are extensive studies reporting on the time-dependent behavior of intact rocks; however, once the rock fails, it is extremely difficult to maintain a constant load (Peng 1973), resulting in limited information about the time-dependent property of failed rock. Chapter 3 proposed and verified the method of using relaxation test to determine the time-dependent property of intact and failed rock. In Chapter 4, this method determined the viscous parameters in the pre-failure and post-failure region of marble based on the test results in literature. However, the attempt to study the time-dependent behavior in the post-failure region was unsuccessful due to the failure in capturing strain-softening process with axial-displacement control mode in Chapter 3.

This chapter used relaxation test to investigate the time-dependent behavior of intact and failed sandstone under unconfined condition. A few unconfined compression tests were first conducted to determine the mechanical property of the sandstone specimens. The strength and lateral deformation at 
failure were summarized, based on which the specific points to start the relaxation tests in the pre-failure region and post-failure region were determined. Two groups of unconfined compression test with relaxation tests in the pre-failure and post-failure region were then conducted to study the time-dependent behavior of intact and failed specimens. The test results were analyzed based on the failure condition, stress-strain curve, relaxation curve and the variation in the time-dependent property. Finally, the time-dependent property of intact and failed coal measure rocks was analyzed based on the laboratory test in Chapter 3. The laboratory test results provide the parameters for the numerical simulations in the following two chapters.

\subsection{THE TIME-DEPENDENT BEHAVIOR OF INTACT AND FAILED SANDSTONE SPECIMENS}

\subsubsection{Experimental procedure}

(1) Specimen preparation and mechanical properties

Cores were drilled from sandstone blocks and specimens with $5.08 \mathrm{~mm}$ (2 in.) diameter were prepared in the rock mechanics laboratory at West Virginia University following ASTM standard. An MTS servocontrolled compression testing system was used to perform the laboratory test.

As the axial-displacement control mode failed to capture the strain softening process in the unconfined compression tests in Chapter 3, the tests in this chapter used circumferential strain control mode to control the failure process of the specimens. First, axial displacement control mode applied the load onto the specimens with a displacement magnitude of $0.76 \mathrm{~mm}(0.03 \mathrm{in}$.). Circumferential strain control mode then controlled the failure of the specimens. The mechanical behavior of sandstone was determined from four unconfined compression tests with this testing methodology. The tests stopped when the circumferential deformation exceeded the $2.54 \mathrm{~mm}(0.1 \mathrm{in}$.) limit.

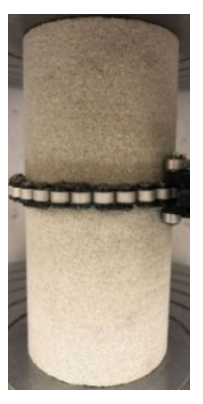

(a) Before test

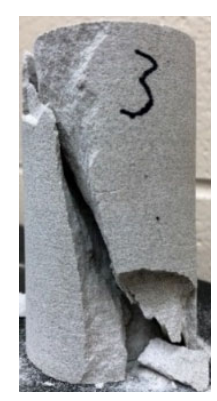

(b) After test

Fig. 5.1. Photos of specimen before and after unconfined compression test

Figs. 5.1, 5.2, and 5.3 show the typical failure mode, stress-strain curves and stress-lateral deformation curves for the unconfined compression tests, respectively. Table 5.1 summarizes the strength and lateral deformation at failure. Fig. 5.1 illustrates that the failure mode of the sandstone specimen is shear failure with a major shear failure plane. As shown in Fig. 5.2, the rock shows Class II behavior in the post-failure region, meaning that the rock is unstable in the post-failure region (Mishra and Nie, 2013). In addition, Fig. 5.2 and Table 5.1 show that there is variation in the rock strength. The strength values vary from $53.06 \mathrm{MPa}$ 
to $61.85 \mathrm{MPa}$ with a variation of $8.79 \mathrm{MPa}$. The sandstone specimens had an average strength of 58.12 $\mathrm{MPa}$. There is also variation in the circumferential deformation when failure occurs, as shown in Fig. 5.3. The circumferential deformation ranges from $0.277 \mathrm{~mm}$ to $0.427 \mathrm{~mm}$ at peak stress values.

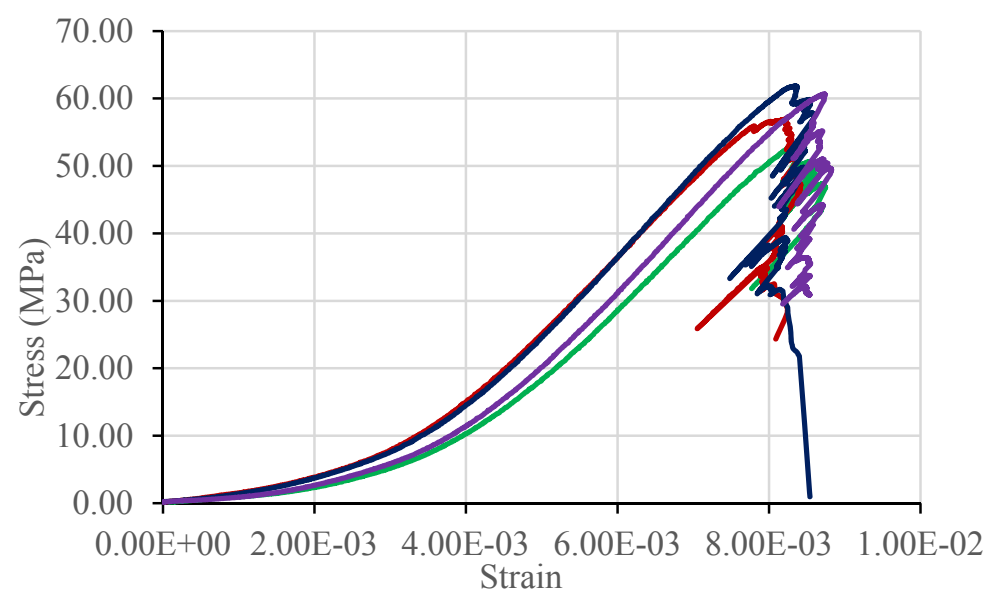

Fig. 5. 2. Stress-axial strain curve for unconfined compression test

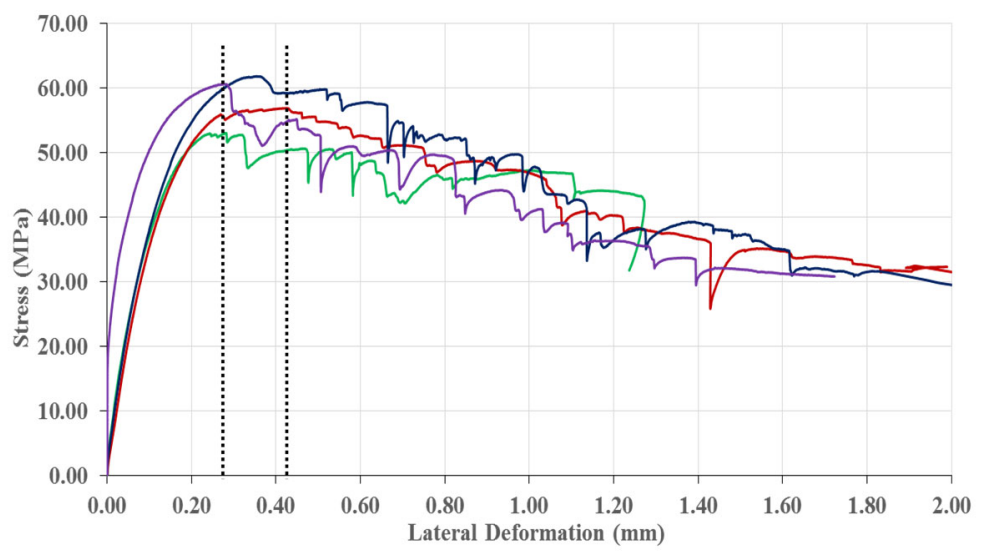

Fig. 5.3. Stress-lateral deformation curve for unconfined compression tests

Table 5.1 Unconfined compression test result summary

\begin{tabular}{|c|c|c|c|}
\hline Test No. & $\begin{array}{l}\text { Strength } \\
\text { (MPa) }\end{array}$ & $\begin{array}{c}\text { Average } \\
\text { Strength (MPa) }\end{array}$ & $\begin{array}{l}\text { Lateral deformation } \\
\text { at failure (mm) }\end{array}$ \\
\hline 1 & 53.06 & \multirow{4}{*}{58.12} & 0.277 \\
\hline 2 & 56.92 & & 0.427 \\
\hline 3 & 61.85 & & 0.353 \\
\hline 4 & 60.66 & & 0.277 \\
\hline
\end{tabular}

(2) Relaxation test methodology

The study designed the laboratory test procedure based on the unconfined compression test results. In the first step, the axial-displacement control mode was used to load the specimens. When the specimens were close to failure and in the post-failure region, the control mode changed to circumferentialdeformation control to get a complete stress-strain curve. For the MTS testing system, when the control 
mode is set as lateral deformation, only the lateral deformation value can be used as the limit value to trigger the relaxation test. According to the unconfined compression test results, the specimens failed with lateral deformation between $0.28 \mathrm{~mm}$ and $0.43 \mathrm{~mm}$. It was designed to perform one relaxation test in the prefailure region and another in the post-failure region. One circumferential displacement value of $0.216 \mathrm{~mm}$ (smaller than $0.28 \mathrm{~mm}$ ) initiated the first relaxation test in the pre-failure region. For the relaxation test in the post-failure region, this study used two lateral deformation values of $0.762 \mathrm{~mm}$ and $1.270 \mathrm{~mm}$. The purpose of choosing these two values was to begin the relaxation test at different failure states in the postfailure region: one is closer to the peak while the other closer to the residual. Therefore, there were two groups of tests. For the first group of tests, one relaxation test initiated with a circumferential displacement value of $0.216 \mathrm{~mm}$ and the other with a circumferential displacement value of $0.762 \mathrm{~mm}$. The second group of tests used circumferential displacement values of $0.216 \mathrm{~mm}$ and $1.270 \mathrm{~mm}$ to initiate the relaxation tests. As a result, these tests investigated the time-dependent property of sandstone with one pre-failure state and two post-failure states.

In determining the duration of the relaxation test, this study conducted a few relaxation tests on the sandstone specimens in the post-failure region. The lateral deformation exceeded the limit of the circumferential strain gauge in about half an hour. Therefore, the duration for the relaxation test was 30 minutes for both in the pre-failure region and in the post-failure region. Fig. 5.4 shows the laboratory test procedure and Table 5.2 summarizes the laboratory test plan.

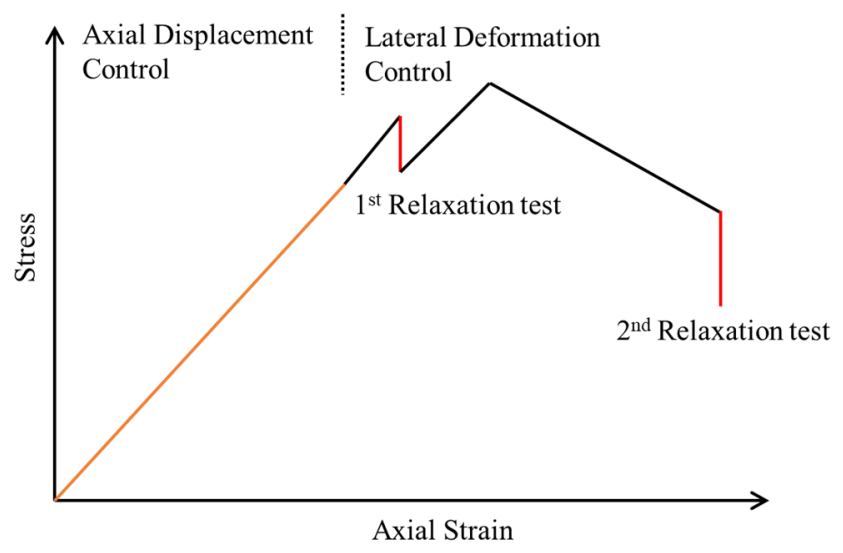

Fig. 5.4. Illustration of the relaxation test procedure

(The orange curve represents the axial displacement control loading part; the black curve represents the lateral control loading part; and the red curves are relaxation curves)

Table 5.2 Summary of laboratory test plan

\begin{tabular}{|c|c|c|c|c|}
\hline $\begin{array}{l}\text { Group } \\
\text { No. }\end{array}$ & $\begin{array}{l}\text { Test } \\
\text { No. }\end{array}$ & $\begin{array}{l}\text { Displacement limit of axial } \\
\text { displacement control ( } \mathrm{mm})\end{array}$ & $\begin{array}{c}\text { Lateral deformation } \\
\text { initiating } 1^{\text {st }} \text { relaxation }(\mathrm{mm})\end{array}$ & $\begin{array}{c}\text { Lateral deformation initiating } \\
2^{\text {nd }} \text { relaxation }(\mathrm{mm})\end{array}$ \\
\hline \multirow{3}{*}{1} & 5 & \multirow{6}{*}{0.76} & \multirow{3}{*}{0.216} & \multirow{3}{*}{0.762} \\
\hline & 6 & & & \\
\hline & 7 & & & \\
\hline \multirow{3}{*}{2} & 8 & & \multirow{3}{*}{0.216} & \multirow{3}{*}{1.270} \\
\hline & 9 & & & \\
\hline & 10 & & & \\
\hline
\end{tabular}




\subsubsection{Results of laboratory test on sandstone specimens}

The unconfined compression tests with relaxation test were divided into two groups. The only difference between these two groups was the lateral deformation initiating the second relaxation test in the post-failure region. A total of six specimens were tested with relaxation test and the test results were analyzed from their stress-strain behavior, failure condition, and the variation in the viscous property.

\section{(1) Failure condition}

After the second relaxation tests, this study examined the failure conditions of the specimens and observed two typical failure conditions. Test No. 7 and No. 9 are examples of this phenomenon. Figs. 5.5 and 5.6 show the specimen photos taken before and after the tests. All the tests terminated when the lateral deformation reached the $2.54 \mathrm{~mm}$ limit of the circumferential strain gauge. The photos illustrate that the failure of the specimens was shear failure with a major failure plane. However, there is difference between them. For the test of No. 6, 9 and 10, there was a major shear failure plane persistent through the specimens, as shown in Fig. 5.6. While the major failure plane did not penetrate cross the whole specimens for test of No. 5, 7 and 8, as shown in Fig. 5.5.

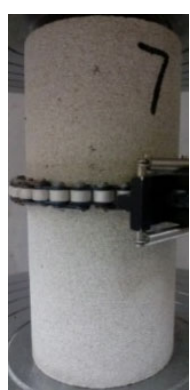

(a) Before test

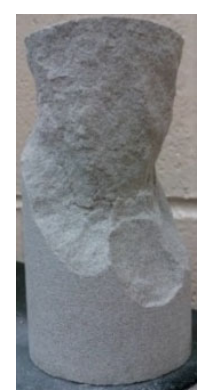

(b) After test

Fig. 5.5. Specimen pictures for test No. 7 with non-persistent major failure plane

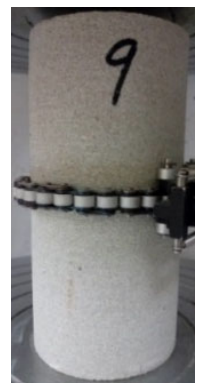

(a) Before test

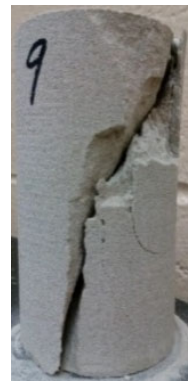

(b) After test

Fig. 5.6. Specimen pictures for test No. 9 with persistent major failure plane

Table 5.3 summarizes the failure conditions of the specimens. Three relaxation tests started with a circumferential deformation of $0.762 \mathrm{~mm}$ and the others with $1.270 \mathrm{~mm}$ circumferential deformation. It was likely that larger circumferential deformation would indicate more failure within the specimen and initiating the relaxation tests in the post-failure region at different circumferential deformation values would assist in studying the relaxation behaviors at different failure conditions. However, Table 5.3 shows that 
there is persistent failure with $0.762 \mathrm{~mm}$ circumferential deformation and non-persistent failure with 1.270 $\mathrm{mm}$ circumferential deformation, meaning that the two circumferential deformation values cannot differentiate two failure condition. The stress values initiating the second relaxation test also show that, except in No. 7 (which had a higher initiating stress value), all the other tests initiated the second relaxation test with a stress value between $45 \mathrm{MPa}$ and $50 \mathrm{MPa}$. Therefore, the results show that the circumferential deformation is not a good indicator of the failure condition. Due to the limit of the circumferential strain gauge, the difference between these two circumferential deformation values is so small that it cannot differentiate two different failure conditions.

Table 5.3 Summary of failure condition

\begin{tabular}{|c|c|c|}
\hline $\begin{array}{c}\text { Test } \\
\text { No. }\end{array}$ & $\begin{array}{c}\text { Lateral deformation starting } \\
\text { relaxation }(\mathbf{m m})\end{array}$ & Failure Condition \\
\hline $\mathbf{5}$ & & Non-persistent failure \\
\hline $\mathbf{6}$ & \multirow{2}{*}{0.762} & Persistent failure \\
\hline $\mathbf{7}$ & & Non-persistent failure \\
\hline $\mathbf{8}$ & & Non-persistent failure \\
\hline $\mathbf{9}$ & \multirow{2}{*}{1.270} & Persistent failure \\
\hline $\mathbf{1 0}$ & & Persistent failure \\
& & \\
& &
\end{tabular}

(2) Stress-strain behavior

Fig. 5.7 shows the stress-strain curves for the tests of the first group-No. 5, No. 6, and No. 7, with certain notable features. First, the relaxation test ran successfully on failed specimens with circumferentialdeformation control. Chapter 3 verified the method of using relaxation test to determine the time-dependent parameters in the pre-failure region by comparing the parameters determined with creep test and relaxation test; this study attempted to extend this method to the post-failure region by assuming viscoelasticity in the post-failure region. However, the axial-displacement control model failed to capture the strain-softening process and, in turn, no relaxation tests could take place during strain-softening process. This chapter then changed the laboratory test on sandstone to circumferential-displacement control mode. When close to failure, the specimens start to dilate. The circumferential-displacement control mode regulates the dilating or failing process of the specimens by adjusting the loading rate in the axial direction, preventing the violent failure with axial-displacement control. As a result, this successfully captured the strain-softening process and relaxation tests could run in the post-failure region of the unconfined compression test. However, it is notable that only one relaxation test was able to be conducted during the strain-softening process; due to the range of the circumferential strain gauge, it could only capture part of the strain-softening process. As shown in Figs. 5.2 and 5.7, the stress-strain curve did not reach the residual stage, and the tests terminated after meeting the maximum range of the circumferential strain gauge. 


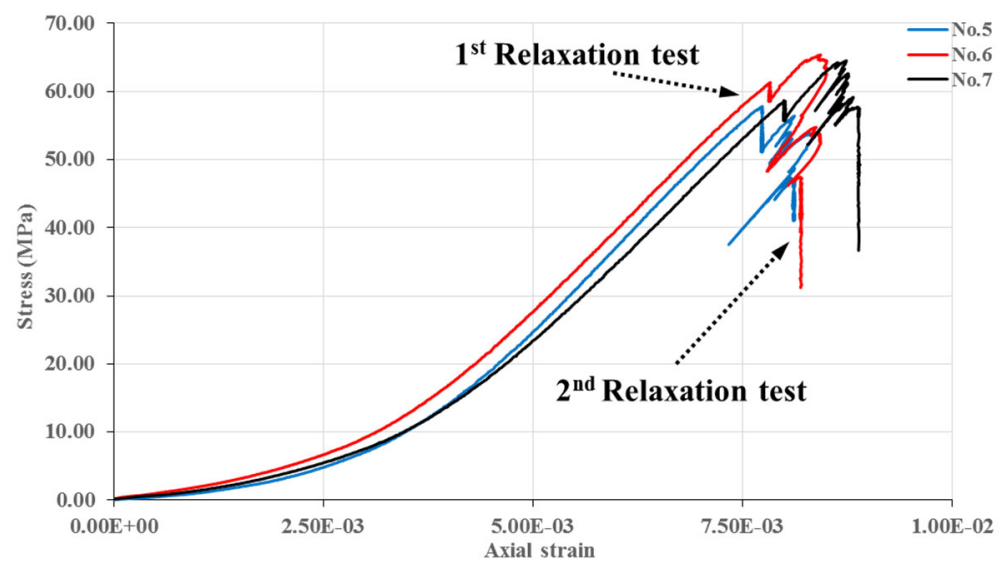

Fig. 5.7. The stress-strain curves for test No. 5, No. 6 and No. 7

In addition, Fig. 5.7 shows that the stress relaxed from a specimen depends on the stress condition initiating the relaxation test. During a relaxation test, the axial strain value is constant, and the stress is relaxing from the specimen. The stress-axial-strain curve for a relaxation test is a vertical line, and the length of the line represents the magnitude of relaxed stress. Fig. 5.7 illustrates that the length of the vertical line for the first relaxation test of test No. 5 is longer than those of No. 6 and No. 7, indicating that more stress relaxed from test No. 5 than the other two.

On one hand, comparing the stress values initiating the relaxation test in the pre-failure region shows that the absolute stress level is lowest for test No. 5, where more stress was relaxed. It indicates that the absolute stress levels initiating the relaxation test are not the key factor affecting the stress relaxation rate.

On the other hand, observing the stress levels initiating the relaxation test and the peak stress values, the relaxation test for No. 5 ran at its peak value while the initiating stress levels for the other two tests were much lower than their peak values. This observation indicates that more stress relaxes from the specimen applied with a stress value closer to its strength. To better show this relation, the stress initiating the relaxation tests in the pre-failure region and the relaxed stress during the relaxation tests were normalized to its peak stress value and their relation is shown in Fig. 5.8. The stress ratio initiating relaxation represents the ratio of the stress value starting relaxation to its peak stress value, which represents the relative stress level; the relaxed stress ratio, in turn, is the ratio of relaxed stress value to its peak stress value. Fig. 5.8 demonstrates that more stress relaxed from the specimens when the relaxation test initiates at a stress values closer to its peak strength. When the relaxation tests were initiated almost at the peak $(100 \%$ of the peak stress), more than $10 \%$ of the peak stress relaxed in 30 minutes. About $4 \%$ of the peak stress relaxed for the same duration when the initiating stresses were below $94 \%$ of the peak stress values. Therefore, the stress relaxation is more sensitive to the relative stress level, rather than the absolute stress level. 


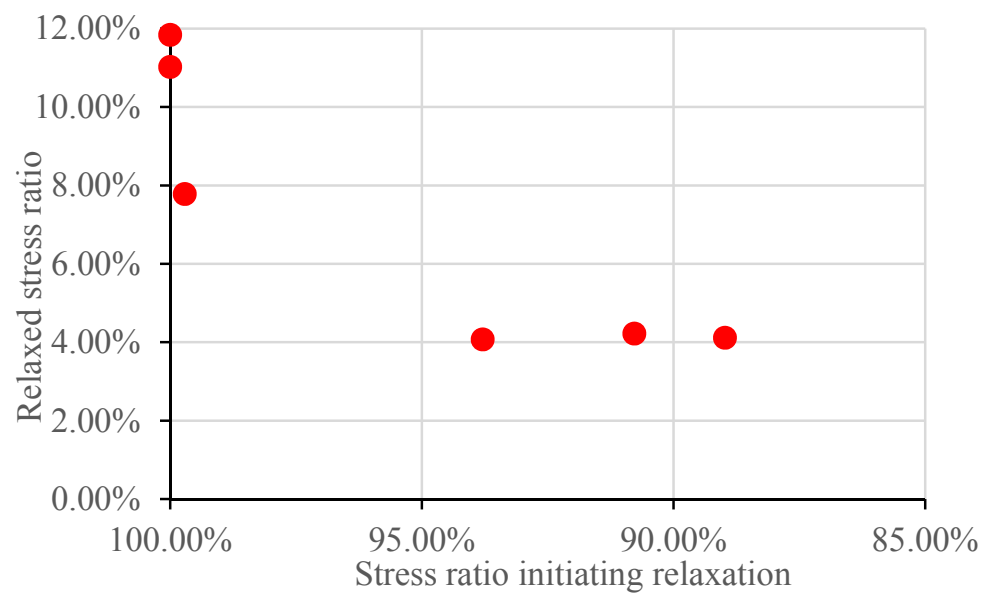

Fig. 5.8. Relation between stress ratio starting relaxation and relaxed stress ratio in the pre-failure region

Furthermore, Fig. 5.7 shows that the stress relaxed from the specimen changes with failure condition. The length of the relaxation curves in the post-failure region are longer than those in the pre-failure region, indicating that more stress relaxed from failed specimens than from those that are intact. This is the variation in the time-dependent property for rocks before and after failure that was mentioned in previous chapters. Test No. 6 is an example of this variation. Fig. 5.9 shows the relation between time and stress ratio during the relaxation test in the pre-failure and post-failure region. The stress ratio is ratio of stress values to the stress value at the beginning of the relaxation test. Fig. 5.9 shows that more stress relaxes from the failed specimen than from the intact specimen. For the intact specimen, about $5 \%$ of the stress was relaxed from the specimen over 30 minutes. About $17 \%$ of the stress relaxed from the failed specimen before the sudden failure of the specimen. In a shorter time-frame, more stress was relaxed from the failed specimen.

Aside from the difference in the magnitude of relaxed stress between intact and failed specimens, the relaxation behaviors of intact and failed specimens are also different. The stress relaxation curve in the prefailure region shows a typical and smooth stress relaxation behavior; for those in the post-failure region, however, the stress relaxation demonstrates staircase type behavior with abrupt plunges in the stress after a stage of smooth relaxation. Another stage of smooth relaxation followed the abrupt plunges and, at some point, a large abrupt stress plunge occurred, leading to the termination of the test. In the pre-failure region, the specimen is intact, and the measured stress during a relaxation test represent the response of the intact specimen with typical smooth relaxation behavior. The sandstone specimens, conversely, have a major shear failure plane and they are not intact. The measured stress during the relaxation test represents the overall response of intact rock pieces and the failure plane.

Two phenomena can result in the stepwise relaxation behavior in the post-failure region. The first one is the propagation of the non-persistent failure plane during the stress relaxation. Although the stress was relaxing, there was still high stress concentration at the tip of the cracks and the interaction between the cracks can lead to the propagation of the failure plane and release the stress suddenly. After the sudden release of the stress, the further failure propagation is inhibited and results in smooth stress relaxation. In addition, the second one is shear-off of the asperities along the major failure plane. At the beginning of the relaxation test in the post-failure region, the asperities along the failure plane interlock and induce stress 
concentration. These asperities shear off at some point during the smooth stress relaxation and induce a sudden slip. At the same time, the stress releases and the specimen reach a stable stage. Another sudden slip could occur at some point after a stage off smooth stress relaxation and may lead to the unstable failure of the specimen. This behavior is similar to the post-seismic deformation, which is primarily due to the afterslip and relocking at different parts of the main rupture zone (Klein et al. 2016). As the confining stress for triaxial condition inhibit the sliding along the failure plane, it is likely that the confining stress will inhibit the sudden slip during the relaxation test in the post-failure region, as well as obtaining smooth relaxation curves from failed specimens.

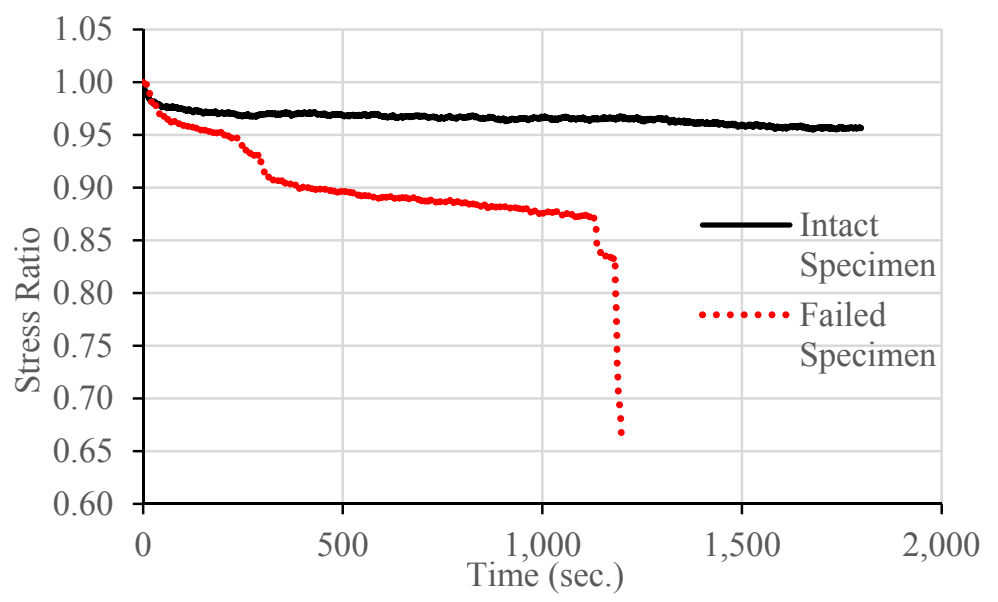

Fig. 5.9. The relation between time and stress ratio during the relaxation tests in the pre-failure and post-failure region for Test No. 6

(3) Variation in the time-dependent property of sandstone

This study examined the variation in the time-dependent property of sandstone by determining the viscous parameters in the pre-failure and post-failure region based on the proposed method in Chapter 3. Table 5.4 summarizes the averaged viscous parameters for intact and failed sandstone and the ratio of each of the parameters.

Table 5.4 Summary of the averaged viscous parameters for intact and failed rock

\begin{tabular}{|c|c|c|c|}
\hline & Intact specimen & Failed specimen & Ratio \\
\hline $\boldsymbol{\eta}_{\mathbf{k}}\left(\mathbf{G p a}{ }^{\mathbf{s}}\right)$ & $8.18 \mathrm{E}+03$ & $2.16 \mathrm{E}+03$ & 0.26 \\
\hline $\mathbf{E}_{\mathbf{k}}(\mathbf{G P a})$ & 157.69 & 172.48 & 1.09 \\
\hline $\boldsymbol{\eta}_{\mathbf{m}}\left(\mathbf{G p a}{ }^{\mathbf{s}} \mathbf{s}\right)$ & $2.52 \mathrm{E}+05$ & $4.64 \mathrm{E}+04$ & 0.18 \\
\hline
\end{tabular}

Table 5.4 shows significant difference in the viscous parameters between intact and failed specimens. From the ratio of the viscous parameters in the pre-failure region to those in the post-failure region, it is notable that the viscous parameters of failed specimens are much smaller than those of the intact ones, except the Kelvin modulus. The relaxation curves resulted from the viscous parameters in Table 5.4 are plotted in Fig. 5.10, where the difference in the relaxation behavior can be observed. The relaxation behavior in the first stage of the relaxation curve is mostly determined by the viscous parameters for the Kelvin part of Burgers model. Due to the significant difference in $\eta_{\mathrm{k}}$ and minor difference in $E_{\mathrm{k}}$, there is significant difference in the slope of the relaxation curve in the first stage and minor difference in the 
magnitude of stress relaxed in the first stage. As a result, initiating from the same stress level, more rapid stress relaxation occurs in the post-failure region, requiring shorter time to reach the second stage. In addition, the variation in the parameter of $\eta_{\mathrm{m}}$ determines the increase in the difference of relaxed stress in the second stage. Although there is almost no difference in the stress starting the second stage, the difference in the relaxed stress increases with time. Significant difference can be the result of the difference in the stress relaxation rate for a long-term situation.

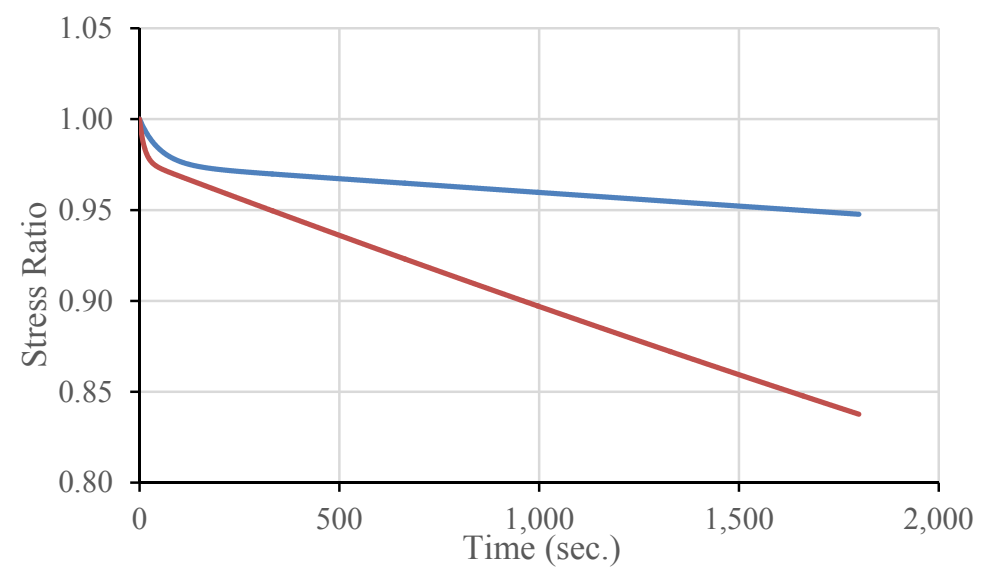

Fig. 5.10. Relaxation curves resulted from different viscous parameters

This study also determined and compared the viscous parameters based on the failure conditions in the post-failure region. The purpose of using two circumferential displacement values in the post-failure region was to investigate the relaxation behaviors at two different failure conditions with the assumption that more circumferential displacement indicates more failure inside the specimen. However, the test results show that all of the relaxation tests in the post-failure region were initiated with a stress value between $45 \mathrm{MPa}$ and $50 \mathrm{MPa}$. It is likely that the circumferential displacement is significant in the post-failure region and that the difference in the circumferential displacement used could not differentiate two failure conditions. This limit is due to the circumferential strain gauge. Rather than the two circumferential displacement values, the two failure conditions-persistent failure and non-persistent failure-after the second relaxation test were used to differentiate the failure conditions. Table 5.5 summarizes the viscous parameters determined based on the failure condition.

Table 5. 5 Summary of the averaged viscous parameters based on the failure condition

\begin{tabular}{|c|c|c|c|c|}
\hline State & \multicolumn{2}{|c|}{ Pre-failure region } & \multicolumn{2}{c|}{ Post-failure region } \\
\hline Parameter & $\begin{array}{c}\text { Non-persistent } \\
\text { Failure }\end{array}$ & $\begin{array}{c}\text { Persistent } \\
\text { Failure }\end{array}$ & $\begin{array}{c}\text { Non-persistent } \\
\text { Failure }\end{array}$ & $\begin{array}{c}\text { Persistent } \\
\text { Failure }\end{array}$ \\
\hline $\boldsymbol{\eta}_{\mathbf{k}}(\mathbf{G p a} * \mathbf{s})$ & $8.94 \mathrm{E}+03$ & $6.87 \mathrm{E}+03$ & $2.40 \mathrm{E}+03$ & $2.40 \mathrm{E}+03$ \\
\hline $\mathbf{E}_{\mathbf{k}}(\mathbf{G P a})$ & 124.89 & 169.34 & 163.37 & 195.16 \\
\hline $\boldsymbol{\eta}_{\mathbf{m}}(\mathbf{G p a} \mathbf{s})$ & $2.10 \mathrm{E}+05$ & $2.95 \mathrm{E}+05$ & $5.34 \mathrm{E}+04$ & $3.98 \mathrm{E}+04$ \\
\hline
\end{tabular}

Table 5.5 shows that there is minor difference in the viscous parameters for the specimens with different failure conditions. In the pre-failure region, the specimens were intact sandstone and there should not have been significant variation in the viscous parameters. The viscous parameters still show minor difference or variation in the pre-failure region. In the post-failure region, the specimens were categorized into two 
groups based on the failure condition. Comparisons of each viscous parameter in the post-failure region show minor difference. If the variation in the viscous parameters in the pre-failure region is taken into account, the conclusion is that there is no difference in the viscous behavior in the post-failure region, regardless of the failure condition.

\subsubsection{Variation in the time-dependent property of coal measure rocks}

Both the literature review and laboratory testing demonstrated insignificant variation in the timedependent properties in the post-failure region. Initially, the variation in the time-dependent properties of marble was investigated based on the relaxation tests in the literature in Chapter 4, and the viscous parameters determined from the various relaxation tests in the post-failure region show insignificant variation. As a result, the study in Chapter 4 ignored the variation in the time-dependent properties in the post-failure region and used one group of viscous parameters to represent the time-dependent properties of failed rock. In addition, the relaxation tests conducted on sandstone in this chapter show insignificant variation in the time-dependent properties, regardless of the failure condition. Therefore, it is rational to ignore the minor variation in the viscous parameters at different stages of the post-failure region.

Laboratory tests in Chapters 3 and 5 determined the viscous parameters of coal measure rocks in the pre-failure and post-failure region. Ignoring the variation in the viscous parameters in the post-failure region, the viscous parameters determined at any stage of the post-failure region could represent the timedependent properties of failed rock. The relaxation tests conducted in the residual stage of the unconfined compression tests determined the viscous parameters for the failed shale, sandyshale and shaly limestone. Table 5.6 summarizes the viscous parameters for coal measure rocks in the pre-failure and post-failure region.

Table 5.6 Summary of mechanical parameters determined from the laboratory tests

\begin{tabular}{|c|c|c|c|c|c|c|}
\hline Rock type & Condition & K (GPa) & $E_{m}(G P a)$ & $\eta_{\mathrm{k}}\left(\mathrm{Gpa}^{*} \mathrm{~s}\right)$ & $E_{k}(G P a)$ & $\eta_{\mathrm{m}}\left(\mathrm{Gpa}^{*} \mathrm{~s}\right)$ \\
\hline \multirow{2}{*}{ Shale } & Pre-failure & \multirow{2}{*}{6.06} & \multirow{2}{*}{3.64} & $3.09 \mathrm{E}+04$ & 42.34 & $6.62 \mathrm{E}+05$ \\
\hline & Post-failure & & & $5.44 \mathrm{E}+03$ & 23.95 & $3.38 \mathrm{E}+05$ \\
\hline \multirow{2}{*}{ Sandyshale } & Pre-failure & \multirow{2}{*}{8.13} & \multirow{2}{*}{4.89} & $8.94 \mathrm{E}+04$ & 106.54 & $2.05 \mathrm{E}+06$ \\
\hline & Post-failure & & & $5.66 \mathrm{E}+03$ & 34.19 & $1.44 \mathrm{E}+06$ \\
\hline \multirow{2}{*}{$\begin{array}{c}\text { Shaly } \\
\text { Limestone }\end{array}$} & Pre-failure & \multirow{2}{*}{12.75} & \multirow{2}{*}{7.58} & $5.99 \mathrm{E}+04$ & 80.17 & $8.25 \mathrm{E}+05$ \\
\hline & Post-failure & & & $4.31 \mathrm{E}+03$ & 28.79 & $2.60 \mathrm{E}+05$ \\
\hline \multirow{2}{*}{ Sandstone } & Pre-failure & \multirow{2}{*}{8.12} & \multirow{2}{*}{4.87} & $8.18 \mathrm{E}+03$ & 157.69 & $2.52 \mathrm{E}+05$ \\
\hline & Post-failure & & & $2.16 \mathrm{E}+03$ & 172.48 & $4.64 \mathrm{E}+04$ \\
\hline
\end{tabular}

\subsection{SUMMARY}

Relaxation test has been proposed and verified as an alternative method to determine the time-dependent property of intact and failed rocks. However, the attempt to conduct relaxation test in the post-failure region during unconfined compression tests failed because the tests were unable to capture the strain-softening process in Chapter 3. Only a few relaxation tests ran successfully in the residual stage. In order to demonstrate that relaxation test can run successfully during strain-softening process and determine the viscous parameters for failed rock, this study conducted unconfined compression tests with relaxation tests 
on sandstone specimens to investigate the time-dependent behavior of intact and failed sandstone under unconfined condition. This section first conducted a few unconfined compression tests to determine the mechanical property of the sandstone specimens; it then summarized the strength and lateral deformation at failure based on which the specific points to start the relaxation tests in the pre-failure and post-failure region were determined. Two groups of unconfined compression test with relaxation tests in the pre-failure and post-failure region were then conducted to study the time-dependent behavior of intact and failed sandstone specimens. For the first group of tests, one relaxation test initiated with a circumferential displacement value of $0.216 \mathrm{~mm}$ and the other with a circumferential displacement value of $0.762 \mathrm{~mm}$. The second group of tests used circumferential displacement values of $0.216 \mathrm{~mm}$ and $1.270 \mathrm{~mm}$ to initiate the relaxation tests. As a result, these tests investigated the time-dependent property of the sandstone in one pre-failure state and two post-failure states.

The test results show that the relaxation behaviors of intact and failed rock specimens are different. The stress relaxation curves in the pre-failure region show a typical, smooth stress relaxation behavior, while, the stress relaxation demonstrates stepped behavior in the post-failure region. The failed specimens experience sudden and large stress drops after a stage of smooth relaxation. As a result, more stress relaxed from the failed specimens than those that are intact. The sudden stress drops are likely induced by the failure propagation or shear-off of asperities along the major shear failure plane.

In addition, tests showed two observable failure conditions, regardless of the circumferential displacement values initiating the second relaxation test. Using two circumferential displacement values in the post-failure region to initiate the second relaxation test did not differentiate the failure conditions. Instead, the failure conditions differentiated due to the persistence of the major failure plane inside of the specimens. Some specimens had persistent failure plane while other had non-persistent failure plane. The viscous parameters determined at different failure conditions did not show significant difference, and their average represents the time-dependent properties of failed rock.

Finally, the relaxation tests in the residual stage helped determine the viscous parameters of failed coal measure rocks. According to the observation that the variation in the time-dependent properties of failed rock were insignificant, it is reasonable to determine the viscous parameters from the relaxation tests at any stage in the post-failure region. Therefore, the relaxation tests in the residual stage determined the viscous parameters for failed shale, sandyshale, and shaly limestone, and the viscous parameters of failed sandstone were determined from the relaxation tests during the strain-softening process. This chapter then summarizes the viscous parameters for various coal measure rocks in the pre-failure and post-failure region. 


\section{CHAPTER 6 \\ DEVELOPMENT OF A VISCOELASTIC-STRAIN-SOFTENING MODEL}

\subsection{INTRODUCTION}

The time-dependent behavior of roof rocks is one key factor affecting the roof stability in underground mines. However, previous studies considered only part of the time-dependent behavior. On one hand, for the time-independent study, the focus centered on two major areas: the failure criterion, which determines whether the rock will fail under certain and/or complex stress condition, and the stability analysis of underground openings with different rock properties and different in-situ stress conditions. Some studies used strain-softening model to simulate the progressive failure. However, the progressive failure is a result of the stress path induced by the underground development, rather than a real time-dependent behavior. Thus, the time-dependent studies ignore the influence of time. On the other hand, the focus of the timedependent study centered on the accumulated strain with time and the failure under creep condition. A creep model with parameters determined from creep tests can simulate the time-dependent deformation of underground structures. Even though the creep model can successfully capture the tertiary stage of the creep curves, it is still within the pre-failure region and the associated creep parameters cannot represent the timedependent property of failed rock.

In actuality, the time-dependent roof failure is the result of progressive propagation of localized failure involving various behaviors, including the time-dependent behavior of intact and failed rock, strainsoftening behavior and strength degradation caused by viscous behaviors. The study on the time-dependent behavior of mine roof should take all these behaviors into consideration.

The time-dependent behavior of rocks can be demonstrated in two forms: creep and relaxation. First of all, rock shows creep behavior with strength degradation occurring during the process. When applying a constant load onto the top of a rock specimen, the specimens continue deforming with time. A laboratory creep test can then obtain the creep curves showing the relation between strain or strain rate and time. The creep model and associated parameters can then be determined by matching the creep curve. Also, the failure of rock specimens during creep tests are known to occur at stress levels lower than the strength of intact rock, indicating that rock strength degrades with time. If strength degradation is not taken into consideration, the strength is constant during a creep test. When the applied stress is lower than the rock strength value, the specimen should keep deforming without failure. The simulations in Chapter 4 have demonstrated the importance of incorporating the strength degradation into the study on the time-dependent stability of mine roof. However, the creep models in 3DEC does not consider the time-dependent strength degradation, and they are unable to reproduce the failure induced by accumulated viscous deformation.

In addition, relaxation is another time-dependent behavior of rocks that can illustrate the variation in the time-dependent property of rocks. After loading the specimen and keeping the displacement constant, the stress within the specimen will gradually drop because of viscous behaviors, resulting in stress relaxation. 
Laboratory creep test is often the preferred method for determining the time-dependent property of rocks. However, once the rock fails, it is extremely difficult to maintain a constant load. Various researchers have used the relaxation test to overcome the challenges posed in constant load tests. Studies have performed relaxation tests successfully at different stages of a complete load-displacement curve. The test results demonstrate that the stress relaxation in the post-failure region is more pronounced than in the pre-failure region. For the same duration, more stress relaxes during the tests in the post-failure region than that in the pre-failure region, demonstrating that there is variation in the time-dependent property; in other words, there is a significant change in the time-dependent property when failure occurs. If viscoelastic behavior is assumed for failed rock, the variation in the viscous parameters can represent the change in the timedependent property. The numerical simulations in Chapter 4 demonstrated the importance of the variation in the time-dependent property in time-dependent stress redistribution and failure propagation.

Furthermore, the time-dependent behavior and strain-softening behavior occur at the same time in the post-failure region. The strain-softening behavior is usually considered to be a time-independent behavior. However, time-dependent behaviors, such as relaxation and creep, can still occur during the strain-softening process and there are different time-dependent properties during the strain-softening process from that in the pre-failure region. This demonstrates the necessity of developing a model that can describe the timedependent behaviors and the strain-softening behavior while considering the variation in the timedependent property and strength degradation.

This chapter proposed a new viscoelastic-strain-softening model to describe the time-dependent and time-independent behaviors of rock in numerical simulations. Burgers model represents the viscoelastic behavior of rock and the viscous parameters vary to include the effect of the variation in the time-dependent property of rock before and after failure. In addition, the strain-softening model describes the behavior of rock in the post-failure region, where the strength decreases with increasing effective shear plastic strain. Similarly, a strength degradation relationship that represents the relation between rock strength and effective viscous strain accounts for the strength deterioration caused by the viscous behaviors. This chapter also explains the procedures to obtain associated parameters and calibrate the model. Finally, a series of laboratory tests were simulated with the developed model for verification purpose, and the results demonstrate that the new model can reproduce the time-dependent, strain-softening behavior observed during laboratory test.

\subsection{DEVELOPMENT OF A NEW VISCOELASTIC-STRAIN-SOFTENING MODEL}

The viscoelastic-strain-softening model is characterized by a viscoelastic-plastic deviatoric behavior and an elasto-plastic volumetric behavior. The viscoelastic behavior is represented by Burgers model where Kelvin cell in series with a Maxwell component, and the plastic model corresponds to a Mohr-coulomb model with non-associated shear and associated tension flow rules. Cohesion, friction angle, dilation angle and tensile strength can soften after the onset of plastic yield.

\subsubsection{Viscoelastic calculation}

The deviatoric behavior is viscoelastic and is described by Burgers model. For the Kelvin unit, 


$$
F=\dot{u}_{k} \eta_{k}+E_{k} u_{k}
$$

where $\dot{u}_{k}$ and $u_{k}$ are strain rate and strain of Kelvin unit, respectively. Fig. 3.5 contains the notation of $F, E_{k}$ and $\eta_{k}$. Writing Eq. 6.1 in finite-difference form,

$$
\bar{F}=\frac{u_{k}^{\prime}-u_{k}^{0}}{\Delta t} \eta_{k}+E_{k} \bar{u}_{k}
$$

where $\bar{F}$ and $\bar{u}_{k}$ correspond to the mean values of $\mathrm{F}$ and $u_{k}$ over the timestep, which have the following forms in Eqs. 6.3 and 6.4, and the superscripts' and ${ }^{\circ}$ denote the new and old values, respectively.

$$
\begin{gathered}
\bar{F}=\frac{F^{\prime}+F^{0}}{2} \\
\bar{u}_{k}=\frac{u_{k}^{\prime}+u_{k}^{0}}{2}
\end{gathered}
$$

Combining Eqs. 6.2, 6.3 and 6.4,

$$
u_{k}^{\prime}=u_{k}^{0}+\left[F^{\prime}+F^{0}-E_{k}\left(u_{k}^{\prime}+u_{k}^{0}\right)\right] \frac{\Delta t}{2 \eta_{k}}
$$

For the Maxwell unit, there is,

$$
\dot{u}_{m}=\frac{\dot{F}}{E_{m}}+\frac{\bar{F}}{\eta_{m}}
$$

where $\dot{u}_{m}$ is the strain rate of the Maxwell unit and $\dot{F}$ is the stress rate. Writing Eq. 6.6 in finitedifference form,

$$
u_{m}^{\prime}=u_{m}^{0}+\frac{F^{\prime}-F^{0}}{E_{m}}+\frac{F^{\prime}+F^{0}}{2 \eta_{m}} \Delta t
$$

Finally, the combination of strains from the Kelvin unit and Maxwell unit is the total strain.

$$
u^{\prime}-u^{0}=u_{m}^{\prime}-u_{m}^{0}+u_{k}^{\prime}-u_{k}^{0}
$$

By combining Eqs. 6.5, 6.7 and 6.8,

$$
F^{\prime}=\frac{1}{X}\left[u^{\prime}-u^{0}+Y F^{0}-\left(\frac{B}{A}-1\right) u_{k}^{0}\right]
$$

where

$$
\begin{gathered}
A=1+\frac{E_{m} \Delta t}{2 \eta_{k}} \\
B=1-\frac{E_{m} \Delta t}{2 \eta_{k}} \\
X=\frac{1}{E_{m}}+\frac{\Delta t}{2 \eta_{m}}+\frac{\Delta t}{2 A \eta_{k}} \\
Y=\frac{1}{E_{m}}-\frac{\Delta t}{2 \eta_{m}}-\frac{\Delta t}{2 A \eta_{k}}
\end{gathered}
$$


Several things are notable in the expressions within the brace in Eq. 6.9. The first two items represent the total strain increment, which the 3DEC software can calculate, and the second item describes the old stress value. The third item represents the old Kelvin strain value, meaning that the calculation of new stress at each timestep should be dependent on the Kelvin strain values in the previous timestep (aside from the strain increment and old stress value). Therefore, the response of Burgers model depends on strain history and the state variable recording history information is $u_{k}$, which has an evolution equation derived from Eq. 6.5,

$$
u_{k}^{\prime}=\frac{1}{A}\left[B u_{k}^{0}+\left(F^{\prime}+F^{0}\right) \frac{\Delta t}{2 \eta_{k}}\right]
$$

At each timestep, one should calculate the new value of Kelvin strain after new stress calculation. This value will assist in calculating the new stress values in the next timestep.

The above calculation only accounts for one stress component; in the following calculation, tensor accounts for all the stress and strain components. The symbols $\sigma_{i j}$ and $\epsilon_{i j}$ represent stress and strain, respectively. The symbols $S_{i j}$ and $e_{i j}$ are deviatoric stress and strain while $\sigma_{0}$ and $e_{v o l}$ represent volumetric stress and strain. They have the following relations.

$$
\begin{gathered}
S_{i j}=\sigma_{i j}-\sigma_{0} \delta_{i j} \\
e_{i j}=\epsilon_{i j}-\frac{e_{v o l}}{3} \delta_{i j}
\end{gathered}
$$

where

$$
\begin{gathered}
\sigma_{0}=\frac{\sigma_{k k}}{3} \\
e_{v o l}=\varepsilon_{k k}
\end{gathered}
$$

The superscripts $k, m$, and $p$ represent the contributions of Kelvin, Maxwell and plastic components to stresses and strains, respectively. Also, the superscripts $n$ and $o$ denote new and old values. In the form of tensor, Eq. 6.9 and Eq. 6.14 are as follows,

$$
\begin{aligned}
& S_{i j}^{n}=\frac{1}{a}\left[\Delta e_{i j}+b S_{i j}^{o}-\left(\frac{B}{A}-1\right) e_{i j}^{k, o}\right] \\
& e_{i j}^{k, n}=\frac{1}{A}\left[B e_{i j}^{k, o}+\frac{\Delta t}{4 \eta^{k}}\left(S_{i j}^{n}+S_{i j}^{o}\right)\right]
\end{aligned}
$$

where

$$
\begin{gathered}
A=1+\frac{E^{k} \Delta t}{2 \eta^{k}} \\
B=1-\frac{E^{k} \Delta t}{2 \eta^{k}} \\
a=\frac{1}{2 E^{m}}+\frac{\Delta t}{4}\left(\frac{1}{\eta^{m}}+\frac{1}{A \eta^{k}}\right)
\end{gathered}
$$




$$
b=\frac{1}{2 E^{m}}-\frac{\Delta t}{4}\left(\frac{1}{\eta^{m}}+\frac{1}{A \eta^{k}}\right)
$$

In elastic/viscoelastic range, calculating the new volumetric stress as follows,

$$
\sigma_{0}^{n}=\sigma_{0}^{o}+K \Delta e_{v o l}
$$

where $K$ is the bulk modulus.

\subsubsection{Failure criterion and flow rule}

The calculation of new stress assumes that the behavior is viscoelastic, and the calculated new stress is 'trial new stress'. The failure criterion then allows to check whether or not failure occurred. If failure is detected, one can calculate the plastic strain based on the flow rule and make stress correction on the trail stress. Otherwise, the trial stress is the new stress which returns to 3DEC for next timestep.

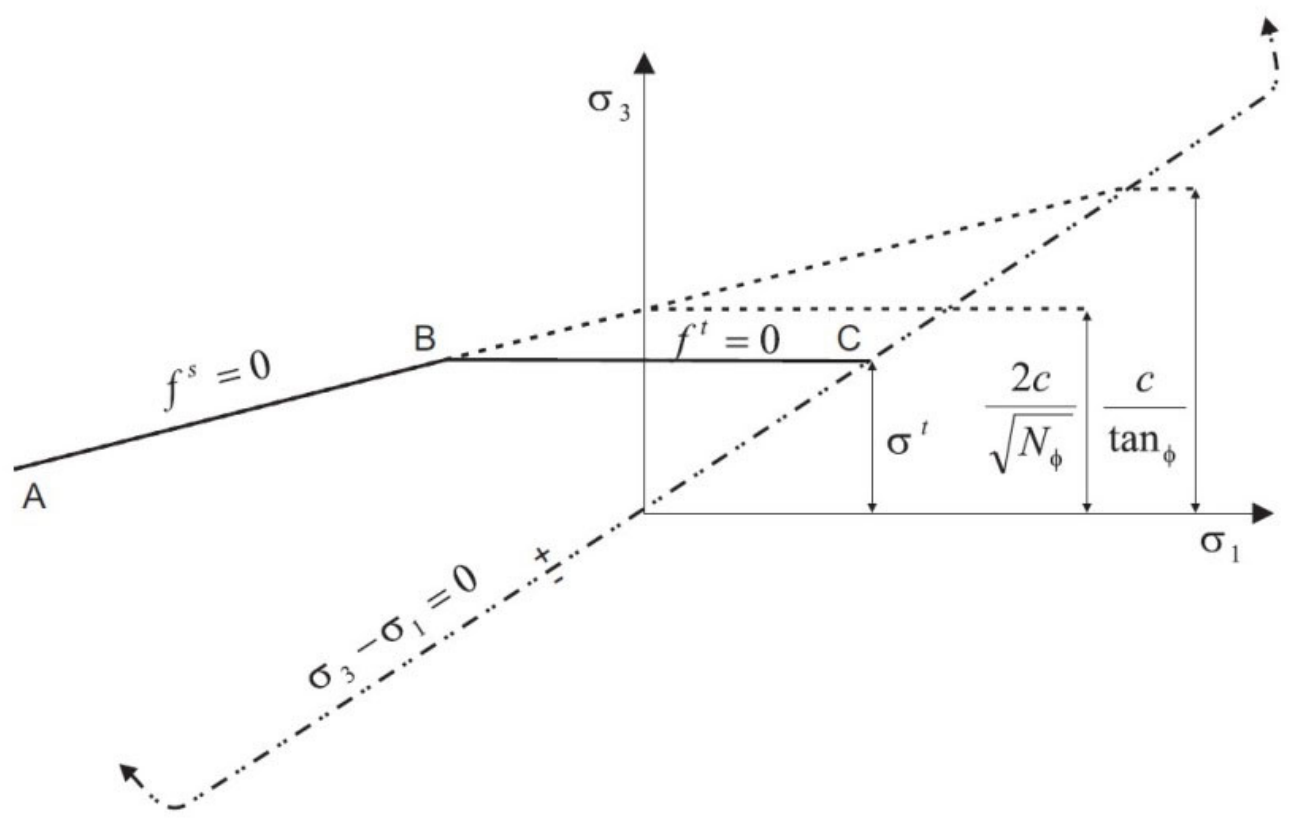

Fig. 6.1. Mohr-Coulomb failure criterion (Itasca 2012)

The failure criterion used in the new model is Mohr-Coulomb failure criterion, which is a composite of shear and tensile criteria, as shown in Fig. 6.1. The yield criterion is $f=0$, and in the principal axes formulation, there is,

Shear yielding:

$$
f=\sigma_{1}-\sigma_{3} N_{\phi}+2 C \sqrt{N_{\phi}}
$$

Tension yielding:

$$
f=\sigma^{t}-\sigma_{3}
$$

where $C$ is cohesion, $\phi$ is friction angle, $\sigma^{t}$ is tensile strength, $\sigma_{1}$ and $\sigma_{3}$ are the minimum and maximum 
principal stresses (compression negative), and $N_{\phi}$ has the following form.

$$
N_{\phi}=\frac{1+\sin \phi}{1-\sin \phi}
$$

It is notable that the tensile strength cannot exceed the $\sigma_{3}$ value corresponding to the intersection point of the straight lines $f^{s}=0$ and $\sigma_{1}=\sigma_{3}$ in the $f\left(\sigma_{1}, \sigma_{3}\right)$ plane. This maximum value is given by

$$
\sigma_{\max }^{t}=\frac{C}{\tan \phi}
$$

Two functions, $g^{s}$ and $g^{t}$, define shear plastic flow and tensile flow rules, respectively. The function $g^{s}$ corresponds to a non-associated flow rule and has the form,

$$
g^{s}=\sigma_{1}-\sigma_{3} N_{\varphi}
$$

where $\varphi$ is dilation angle and

$$
N_{\varphi}=\frac{1+\sin \varphi}{1-\sin \varphi}
$$

The function $g^{t}$ corresponds to an associated law.

$$
g^{t}=-\sigma_{3}
$$

The following technique ensures that the flow rule gives a unique definition. Fig. 6.4 defines a function $h\left(\sigma_{1}, \sigma_{3}\right)=0$. It separates the plastic domain into positive and negative domains, as indicated on the figure and has the form,

$$
h=\sigma_{3}-\sigma^{t}+a^{p}\left(\sigma_{1}-\sigma^{p}\right)
$$

where $a^{p}$ and $\sigma^{p}$ are constants defined as,

$$
\begin{gathered}
a^{p}=\sqrt{1+N_{\phi}^{2}}+N_{\phi} \\
\sigma^{p}=\sigma^{t} N_{\phi}-2 C \sqrt{N_{\phi}}
\end{gathered}
$$

In Fig. 6.2, a point within the $\left(\sigma_{1}, \sigma_{3}\right)$ plane represents one stress value. Once the trial stress meets the failure criteria, it should be present either in domain 1 or 2 , corresponding to negative or positive domains of $\mathrm{h}=0$, respectively. If the stress point falls within domain 1 , shear failure occurs, and the stress point is placed on the curve $f^{s}=0$ using a flow rule derived using the potential function $g^{s}$. If the point falls within domain 2, this will detect tensile failure, and the new stress point conforms to $f^{t}=0$ using a flow rule derived using $g^{t}$. 


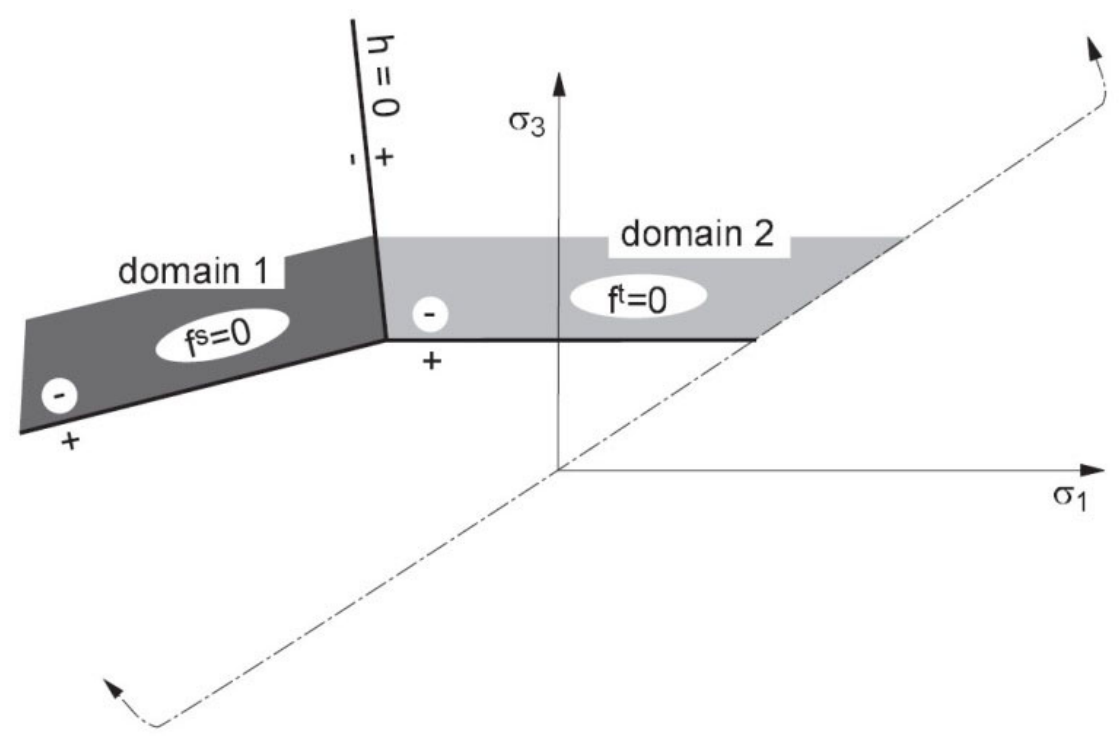

Fig. 6.2. Domains used for the definition of flow rule (Itasca 2012)

\subsubsection{Plastic stress correlation}

When failure occurs, one can partition the total strain rate into three parts.

$$
\dot{e}_{i j}=\dot{e}_{i j}^{k}+\dot{e}_{i j}^{m}+\dot{e}_{i j}^{p}
$$

Basing the calculation of plastic strain rate on the flow rule, it has the following normal form, no matter shear failure or tensile failure.

$$
\begin{gathered}
\dot{e}_{i j}^{p}=\lambda \frac{\partial g}{\partial \sigma_{i j}}-\frac{1}{3} \dot{e}_{v o l}^{p} \delta_{i j} \\
\dot{e}_{v o l}^{p}=\lambda\left[\frac{\partial g}{\partial \sigma_{11}}+\frac{\partial g}{\partial \sigma_{22}}+\frac{\partial g}{\partial \sigma_{33}}\right]
\end{gathered}
$$

If expressed in incremental form, Eq. 6.36 can rearrange as the following,

$$
\Delta e_{i j}-\Delta e_{i j}^{p}=\Delta e_{i j}^{k}+\Delta e_{i j}^{m}
$$

Because the plastic strain has no effect on the stress increment, one should deduct the plastic strain increment from the total strain increment when using strain increment to calculate stress. As a result, the left and right hands of Eq. 6.39 correspond to those of Eq. 6.8, respectively. The new stress calculation after failure occurs, which corresponds to Eq. 6.19, has the following form.

$$
S_{i j}^{n}=\frac{1}{a}\left[\Delta e_{i j}-\Delta e_{i j}^{p}+b S_{i j}^{o}-\left(\frac{B}{A}-1\right) e_{i j}^{k, o}\right]
$$

Corresponding to Eq. 6.25, one can calculate the new volumetric stress when failure occurs as,

$$
\sigma_{0}^{n}=\sigma_{0}^{o}+K\left(\Delta e_{v o l}-\Delta e_{v o l}^{p}\right)
$$

As mentioned before, the visco-elastic assumption provides a basis to calculate the trial new stress, and 
failure criterion is used to test for failure. However, the detection of failure is within principal space, meaning that the first step is to calculate the principal stress components and sort them from the trial new stress. Then the yield function, Eqs. 6.26 and 6.27, is computed. If failure occurs, stress correction should obtain the true value of new stress. This means that Eqs. 6.19 and 6.25 calculate the trial new stress and Eqs. 6.39 and 6.40 calculate the true new stress values if failure criterion is met. The difference between these corresponding equations is the plastic part. One can make the plastic correction by deducting the plastic part from Eqs. 6.19 and 6.25. As with the application of yield function, however, the plastic correction is also made within principal space. Expressing Eqs. 6.9 and 6.40 in principal space and using $\hat{S}_{i}^{n}$ and $\hat{\sigma}_{0}^{n}$ to represent the trial values of principal stresses and calculating volumetric stress with Eqs. 6.19 and 6.25 , one can then make the plastic correction by deducting the plastic part from the trial stresses.

$$
\begin{gathered}
S_{i}^{n}=\hat{S}_{i}^{n}-\frac{1}{a} \Delta e_{i}^{p} \\
\sigma_{0}^{n}=\hat{\sigma}_{0}^{n}-K \Delta e_{v o l}^{p}
\end{gathered}
$$

After stress correction in principal space, the new stresses transfer back to the global space to find the new global stress components, assuming that the occurrence of a plastic correction do not affect the principal directions.

\subsubsection{Variation in the time-dependent property}

Relaxation test results demonstrate that failed rock shows more relaxation than intact rock for the same duration, indicating that the time-dependent parameters in the post-failure region should be much smaller than those in the pre-failure region if assuming viscoelastic behavior in the pre-failure and post-failure region. In addition, the relaxation tests in literature and in this study found that the variation in the viscous parameters determined from the relaxation tests in the post-failure region is relatively small and can be ignored if the difference in pre-failure and post-failure region is taken into consideration. Therefore, it is reasonable to use averaged viscous parameters for the viscoelastic property of the failed rock.

In summary, varying parameters for Burgers model can represent the variation in the time-dependent property at different stages of a complete stress-strain curve. Due to the relatively small variation in the viscous parameters in the post-failure region, averaged parameters can represent the time-dependent property of failed rock. This is the same situation in the pre-failure region where one uses the averaged time-dependent parameters. Therefore, this study will use two groups of time-dependent parameters, one for intact rock and the other for failed rock, to account for the variation in the time-dependent property of rocks before and after failure. A procedure similar to the FISH function in Fig. 4.4 was used in the developed model to vary the viscous parameters when failure occurs.

\subsubsection{Strain-softening behavior}

For the strain softening behavior induced by shear and tensile failure, the two parameters, $\kappa^{\mathrm{s}}$ and $\kappa^{\mathrm{t}}$, are the sum of incremental measures of plastic shear and tensile strain for the zone, respectively. One can calculate the shear and tensile softening increments for the zone as the volumetric average of softening increments over all tetrahedral involved in the zone. 
The shear-softening increment for a particular tetrahedron is a measure of the second invariant of the plastic shear strain increment tensor for the timestep, given as

$$
\Delta \kappa^{s}=\frac{1}{\sqrt{2}} \sqrt{\left(\Delta \varepsilon_{1}^{p s}-\Delta \varepsilon_{m}^{p s}\right)^{2}+\left(\Delta \varepsilon_{2}^{p s}-\Delta \varepsilon_{m}^{p s}\right)^{2}+\left(\Delta \varepsilon_{3}^{p s}-\Delta \varepsilon_{m}^{p s}\right)^{2}}
$$

where $\Delta \varepsilon_{m}^{p s}$ is the volumetric plastic shear strain increment,

$$
\Delta \varepsilon_{m}^{p s}=\frac{1}{3}\left(\Delta \varepsilon_{1}^{p s}+\Delta \varepsilon_{3}^{p s}\right)
$$

And the tensile-softening increment is the magnitude of the plastic tensile strain increment.

$$
\Delta \kappa^{t}=\left|\Delta \varepsilon_{3}^{p t}\right|
$$

The softening of cohesion in terms of the shear parameters is a commonly used method to control the strain-softening behavior of rocks. Softening cohesion was the single softening parameters when using strain-softening model to simulate cutter roof failure in underground coal mines (Gadde and Peng 2005; Ray 2009; Sherizadeh and Kulatilake 2016). The user can provide the relation between cohesion and the shear parameter in the form of tables. Each table contains pairs of values: one for the parameter, and one for the corresponding parameter value. It is assumed that the property varies linearly between two consecutive parameter entries in the table, as shown in Fig. 6.3. The parameter $\Delta \varepsilon^{p t}$ (Eq. 6.45) can describe the softening of the tensile strength in a similar manner.

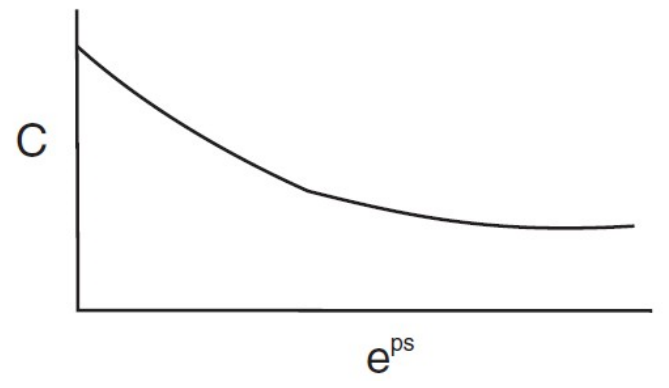

(a) Cohesion variation with plastic strain

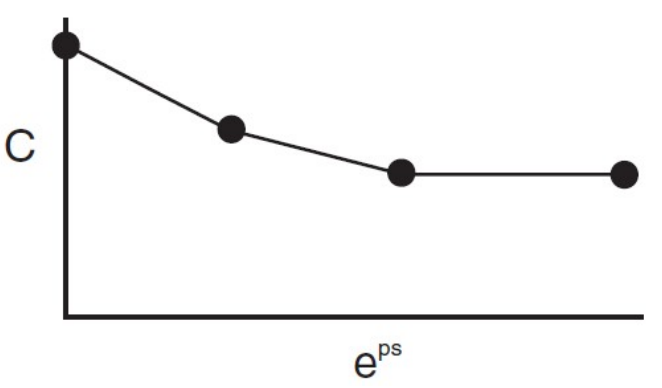

(b) Approximation with segments

Fig. 6.3. Approximation of cohesion variation with plastic train by segments (Itasca 2012)

\subsubsection{Strength degradation}

The strength degradation parameter $\kappa^{v}$ introduced in Chapter 4 was used in the new model to control the strength degradation. Similar to the strain-softening behavior represented by cohesion, the strength degradation is described by friction angle in the new model. The relation between friction angle and the strength degradation parameter can be provided by the user as a table. At each timestep, the model calculates the accumulated effective viscous strain and updates the friction angle value.

Unlike plastic strain, viscous strain in the new viscoelastic-plastic model can recover if the stress unloads. If the friction angle is associated with the real-time effective viscous strain, the friction angle can recover when the calculated effective viscous strain decreases during unloading. To prevent this situation, the 
maximum value of the effective viscous strain in the calculation history is used to determine the real-time friction angle values. This indicates that, once there is viscous strain, the damage is induced; even if the viscous strain recovers later, the damage does not disappear.

\subsubsection{Implementation}

The procedure to calculate the new stress values and to update the material parameters is shown in Fig. 6.4. Trial new stresses $\hat{S}_{i}^{n}$ and $\hat{\sigma}_{0}^{n}$ are computed from Eqs. 6.19 and 6.25 by assuming viscoelastic increments, respectively. The trial stresses are computed by adding stress increments calculated from the total strain increments, $\Delta \varepsilon_{i j}$, to the old stress components. Principal stresses and the corresponding directions are then calculated.

If the stress state does not violate the composite yield criterion (Eqs. 6.26 and 6.27), no plastic flow takes place for this step and the trial stresses gives the new principal stresses. If the stress state meets the failure criterion, one can then calculate the value of $h\left(\sigma_{1}, \sigma_{3}\right)$ function. If that value is negative, shear failure occurs. Otherwise, tensile failure takes place. Eqs. 6.41 and 6.42 can then make plastic correction and obtain the new stress values. Although Eqs. 6.41 and 6.42 relate to shear failure and tensile failure situation, different flow rules (Eqs. 6.30 and 6.32) result in different expressions of $\Delta e_{i}^{p}$ in these two equations. After determining the new principal stresses for one timestep, one can then obtain the new stress components in the system of global axes by assuming that the principal directions have not been affected by the occurrence of plastic flow.

If failure occurs, one can then calculate the increment in softening/hardening parameters for the zone from Eqs. 6.43 or 6.45 after determining the new stress value for the step. Adding these increments into the old values to obtain the new ones, which in turn can update the new values for the zone cohesion, fiction

angle, dilation angle and tensile strength. These renewed parameters can further determine the strength values for next timestep.

In addition, after detecting failure, one must check the viscous parameters of the failed/failing zone. If their values correspond to the values from the pre-failure region, they will need to be assigned new values corresponding to the post-failure region. If their values are equal to those from the post-failure region, no change in the parameters is necessary. The result is that the viscous parameters change to the values from the post-failure region when failure first takes place in that zone. After changing the viscous parameters, the following calculation utilizes them, even when unloading the zone back to elastic range.

It is notable that the new model works as strain-softening model when the creep timestep is zero. By default, the creep timestep is zero and the creep simulation is off. Under this circumstance, only the elastic part of the Maxwell unit is active, and the model is strain-softening model. 


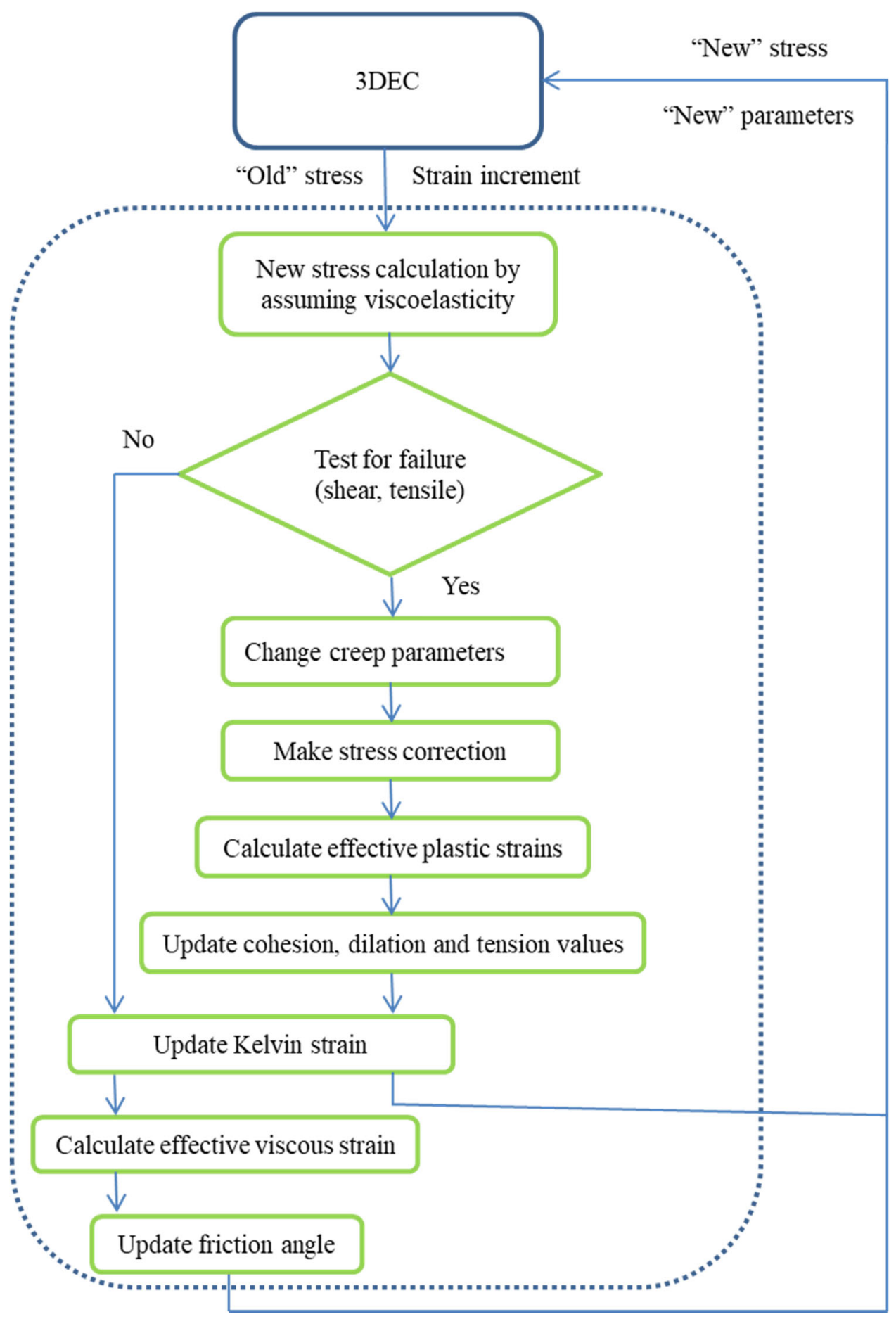

Fig. 6.4. Flowchart showing model implementation

\subsection{PROGRAMMING}

\subsubsection{User-defined model in 3DEC}

To develop user-defined models in 3DEC, the model must be written in $\mathrm{C}++$ and compiled as a DLL file (dynamic link library) that can load whenever needed. The main purpose of the code is to return new stress values, given strain increments from the 3DEC main software at each timestep. As shown in Fig. 6.4, 
the 3DEC main software provides the stress values for each zone from the previous timestep and the calculated strain increments at the current timestep. The user-defined model, in the form of DLL file, calculates the new stress values for the current timestep and updates the associated parameters, which then return back to the 3DEC main software to calculate the new strain increment. Fig. 6.4 details the procedures encapsulated within the dotted line, accomplished by the user-defined model.

$\mathrm{C}++$ language emphasizes the application of object-oriented approach to program structure, using classes to represent objects. An object encapsulates the data associated with the object and is invisible outside of the object. Communication with the object is by member functions that operate on the encapsulated data. This situation is the same in the user-defined model in 3DEC. In actuality, the writing of a user-defined constitutive model in 3DEC is programming to define a class. At the beginning, objects of that class will be created for the zones within 3DEC. All the associated parameters should be obtained from 3DEC software, initialized, and stored within the object when creating the object. While running, 3DEC main software provides stress values and strain increments at each timestep. The member functions within the object calculate new stress values and internal state, and then return them to 3DEC for next timestep. Therefore, the basic functions of the user-defined class are to initialize parameters, obtain parameters from 3DEC commands, calculate new stress values from given strain increments, check failure states, and renew parameters. The member functions within the definition of the class should accomplish each of these goals.

A base class, ConstitutiveModel, is already defined in 3DEC. This base class is an abstract class because it declares a number of member functions. As a result, no one can create an object of this base class; however, it provides a framework for actual constitutive models. All the actual constitutive models, whether built-in or user-defined, are classes derived from the base class. Within the derived classes, one must supply real member functions to replace each one of the pure virtual functions of the abstract class-ConstitutiveModel. Therefore, the main programming work of a user-written constitutive model is to define a class by creating one header file and one implementation file. The header file defines a class by deriving from the base class and declaring all the member functions and member variables used when cycling within the definition of the class. Brief description of each member function should also be present. The implementation file includes the definition of the member functions to implement the operations of an object.

\section{(1) Header file}

The first step is to declare one class for the user-defined constitutive model by deriving from the base class, ConstitutiveModel. The derived class declaration should include the declaration of the member functions and their brief descriptions. The following section lists and describes the member functions and their description that are used in the following constitutive model.

String getName() returns a string containing the name of the constitutive model as the user will refer to it with the $\boldsymbol{Z O N E}$ command. This must be a unique name.

String getFullName() returns a string containing the name of the constitutive model that is used on printout. It may or may not be the same as that given by the getName() member function.

String getProperties() returns a string containing the names of the model properties. The given names 
will be those recognizable by the PROPERTY command. Property names are delimited by commas.

Variant getProperty (UInt $\boldsymbol{n}$ ) returns a value for the model property of sequence number $\boldsymbol{n}$. Variant defines a data type that can represent many different primitive types (e.g., String, Double, Int, etc.). This class passes properties to and from the constitutive model.

String getStates () returns a string containing state names, which represent the failure state. The names are used to printout and in plotting to identify user-defined interval states of the model.

setProperty (UInt n, const Variant $\& \boldsymbol{d V a l}$ ) stores the supplied property values in an appropriate member variable. The value of $\boldsymbol{d} \mathbf{V a l}$ in the function call comes from a 3DEC command of the form PROP $\boldsymbol{n a m e}=\boldsymbol{d V a l}$; the value of $\boldsymbol{n}$ is the sequence number of the property name previously specified by means of a getProperties() call.

Copy (const ConstitutiveModel ${ }^{*} \mathbf{c m}$ ) first calls the base class Copy function and then copies all essential data from the model object pointed to by $\mathbf{c m}$ (assumed to be the same derived class as the current model). It is not necessary to copy data members recomputed when the initialize () function is called. Use dynamic_cast $<$ MyModel $*>(\mathrm{cm})$ to upcast $\mathbf{c m}$.

Initialize (UByte uDim, State *ps) is called once for each model object (i.e., for each full zone) when the 3DEC CYCLE command is given, when large-strain update is performed and at the beginning of the run () method if isValid () returns false. The dimensionality (e.g., this is 3 for 3DEC and 2 for UDEC) is given as $\boldsymbol{u D i m}$ and structure $\boldsymbol{p s}$ contain current information for the zone containing the model object.

Run (BUyte uDim, State *ps) is called for each zone at each cycle form within 3DEC's zone scan. This function will update the stress tensor from strain increments.

Double getConfinedModulus (void) returns a value for its best estimate of the maximum confined modulus, which 3DEC uses to compute the stable timestep.

Double getShearModulus (void) returns a value for its best estimate of the current tangent shearmodulus.

Double getBulkModulus (void) returns a value for its best estimate of the current tangent bulk-modulus.

UInt getMinorVersion () returns the version number of the constitutive model.

Double getStressStrengthRatio (const Sym Tensor \&st) returns the ratio of the passed stress tensor to the yield strength of the zone properties.

Bool supportsHystereticDamping () returns true if supporting hysteretic damping.

Bool supportStressStrengthRatio () return true if supporting stress-strength ratio calculations.

Bool supportPropertyScaling O returns true if supporting property scaling for factor-of-safety calculations.

Aside from these, there are a few member functions declared in the base class and used in the user- 
defined model. The function setValid (UByte dim) indicates by dimension whether initialization needs to be called again. The function canFail () is a bool type function and returns false if SOLVE elastic is in process. The function ConstitutiveModel *clone (void) is used to create a new object of the same class as the current object and returns a pointer to the new object of type ConstitutiveModel. This function is called whenever 3DEC installs the model in a zone.

In addition, the model class definition in header file should contain a constructor that must invoke the base constructor. Constructor initializes the member variables of a class (Malik, 2009). Because there is no access to the member variables of the base class outside the class itself, the initialization of base class member variables can only be done by the base constructor. Therefore, the constructor of the derived class should be able to invoke the base constructor. At the same time, the constructor in the derived class should also initialize the member variables of the derived class. It is recommended that in all cases, the derivedclass constructor should be called with no parameters.

Finally, the Object-Oriented Design in $\mathrm{C}++$ encourages the encapsulation of data, which makes it impossible to access this data outside the object. This is achievable in 3DEC by declaring the member functions to be public while the member variables are to be private.

(2) Implementation file

The implementation file provides the definition of the member functions for the derived class. However, it defines a few functions used for model registration first before other member function. Each user-defined constitutive model is compiled into a DLL file that must be instantiated in the 3 DEC process. By convention, there are four exported functions in a DLL file used as a plug-in to 3DEC. These functions are getName (), getMajorVersion (), getMinorVersion () and createInstance (), which have further explanations below.

The getName () function returns a string always beginning with "model", indicating that the DLL is a constitutive model plug-in. The same string that is returned from the getName () method of the ConstitutiveModel class should be appended to the "model".

The getMajorVersion of function should always be the same. The major version is determined by the base constitutive model DLL and indicated binary compatibility.

The getMinorVersion () function indicates the minor version update of the constitutive model. Increment this value when making changes to the model.

The createInstance () function actually creates and returns an instance of user-defined class. A registry stores this information to create all other instance (via the clone () function).

Following the model registration is the definition of the member functions declared in the header file. These member functions will implement the operations of the object. For a constitutive model, the basic operations include initializing parameters, obtaining parameters from 3DEC commands, calculating new stress values from the given strain increments, checking failure states and renewing parameters. The following functions perform all these operations: getProperties(), getStates O, getProperty (UInt n), setProperty (UInt n, const Variant \&dVal), copy (const ConstitutiveModel *cm), initialize (UByte uDim, 
State *ps), run (BUyte uDim, State *ps). The header file provides the description for each of these functions; there is no list of the detailed code in this section.

Among these member functions, the most important link between 3DEC and a user-defined model is the member function run (unsigned nDim, State *ps). The main task of this function is to compute new stresses from strain increments. In a nonlinear model, it is also useful to communicate the internal state of the model. Fig. 4.6 demonstrates the communication between $3 \mathrm{DEC}$ and the user-defined model during cycling. Accomplishing the work done within the dashed box is mostly within the member function run(). The structure ps contains the current stress components and the computed strain increment components for the zone being processed. Structure is a data type in $\mathrm{C}++$ and is a collection of a fixed number of components in which the components are accessible by name. The components may be of different types (Malik, 2009).

A few important members of this structure (all public) are as follows. These parts will calculate new stresses from given strain increments in each timestep.

Unsigned char sub_zone_ is the sequence number of the sub-zone currently being processed, starting at 0 . This information can scale accumulated sub-zone data correctly.

Unsigned char total_sub_zones_is the total number of sub-zones in the zone currently being processed, including those from all overlays, if present.

Unsigned char overlay_ is the number of times the volume of the current zone is represented.

Unsigned long state_ is model state indicator flag. Specific bits in this flag correspond to unique failure states.

Double getZoneVolume returns the volume of the current full zone.

SymTensor stnE_ is strain increment tensor, input to the constitutive model. For each cycle, 3DEC will calculate the strain increments and then pass the information to the user-written model.

SymTensor $\mathbf{s t n} \boldsymbol{S}_{-}$is the stress tensor. For each cycle, the user-defined model will calculate the new stress based on the strain increments and old stress, and then transfer the updated stress back to 3DEC for next step.

SymTensor is of structure/class type, which defines a $3 * 3$ symmetric tensor. It typically represents stresses and strains. Member access is available through the s11(), s120,s13(), s22(), etc. functions (value parameters), while member modification is available through the rs11(), rs12(), etc. functions (reference parameters). The getEigenInfo() function can obtain the principal directions and values. The helper class Sym TensorInfo allows the user to modify principal values while maintaining principal directions and build up a new Sym Tensor with the result. This is important for obtaining true stress value if failure could occur. Based on the strain increments inputted from 3DEC, the model will calculate a trial stress assuming elastic condition. The trial stress should transfer to principal stress space with the getEigenInfo() function to detect failure condition. If failure occurs, correction on the principal stress values can occur while maintaining the principal directions. The corrected values can then resolve to global axes to obtain the true stress values after considering the plastic state. 
Double getTimeStep returns timestep (input only).

Bool isLarge returns true if the model is currently running in large-strain mode.

Bool isCreep returns true if the creep calculation mode is active.

Bool viscous_ returns true if computing viscous strains for the stiffness-proportional component of Rayleigh damping. Typically, it should be set true for an elastic increment, and false for an increment in which yielding occurs.

Void *getTableIndexFromID (unsigned id) returns table index value. This converts a table number to the index used to access the table.

Double getYFromX (void *index, const Double $\& x$ ) returns a y-value corresponding to the supplies Xvalue from the table identified by its index value. These two functions are important in obtaining the varying cohesion, friction and dilation angle values when strain-softening model is used. In 3DEC commands, each table has an assigned table ID number for cohesion, friction and dilation angle and the getTableIndexFromID() function can covert the table numbers in command to the index used to access the table. Next, one can calculate the accumulated effective shear plastic strains for each zone within plastic state and can then treat this value as an input, $x$-value, for the second function to obtain the strain-softening parameter values from corresponding tables identified by the index value.

Double working_[max_working_l stores values that requires storage between run() calls. These parameters are typically accumulated values for the constitutive models, for example the accumulated effective shear plastic strain in strain-softening model and Kelvin strains in Burgers model.

(3) Creating user-defined model DLLs

In order to create a DLL in Visual Studio 2005, it is first necessary to create a solution, which will contain projects that are essentially a collection of $\mathrm{C}++$ source and header files and their dependencies. Itasca has recommended the following steps and settings to create a new project.

1) Launch Visual Studio 2005 from the Start menu and select FILE/NEW/PROJECT. Under "Project Types", select Visual C++/Win32. Under "Templates", select Win32 project. Select a location and name of the project and press $\mathbf{O K}$.

2) A Win32 Application Wizard dialog will appear. Select "Application setting" on the left. Under "Application type", select DLL. Under "Additional Options", select "Empty Project". Then press FINISH.

3) Copy the files "modelvess.h", "modelvess.cpp", "version.rc" and "version.txt" into the directory created by Visual Studio for your solution and project.

4) In the "Solution Explorer" window of Visual Studio, right-click on the "Header Files" folder and add "modelvess.h". Then right-click on the "Source Files" folder and add "modelvess.cpp". Add the file "version.rc" to the "Resources" folder. Add the file "version.txt" as a project object, "Add Existing Item" from the project context menu. These files are now editable to create the custom constitutive model. 
5) Right-click on the project entry and select "Properties". Make sure that Configuration: reads "Active (Debug)" and Platform: reads "Active (Win32)".

6) Under "Configuration Properties", select the "General" entry. Change the "Output Directory" entry to "\$(ProjectDir)\$(ConfigurationName)".

7) Under "C/C++", select the "General" entry. Add the directories "interface" and "models $\backslash \mathbf{s r c} "$ (separated by ;), both subdirectories of the 3DEC install directory, to "Additional Include Directories". In a typical install, this entry might read "C:IProgram Files\Itascal3dec410linterfaces; C:IProgram Files $\backslash$ Itasca $\backslash 3 d e c 410 \backslash$ models $\backslash$ sre". Also change the "Warning Level" to Level 4 and "Treat Warnings As Errors" to YES.

8) Under "C/C++", select the "Preprocessor" entry. Change "Preprocessor Definitions" so that “_DEBUG" now reads "MODELDEBUG".

9) Under "C/C++", select the "Code Generation" entry. Change "Runtime Library" to "Multithreaded DLL (/MD)".

10) Select "Linker/General". The name of the DLL produced should follow the convention "modelVess001.dIl". The "model" prefix identifies it as a constitutive model. Vess is the name of the constitutive model as returned by the getname() method, and 001 indicates the major version number. This entry should read "\$(OutDir) \modelVessd001.dll". The "d" is to allow one to distinguish a debug version of the model from a release version.

11) Select "Linker/Input". The "Additional Dependencies", for a 64-bit system, should add the files “exe64\lib\base001.lib" and "exe64\lib\conmodel001.lib", separated by a space, and including the 3DEC install directory. In a typical install, this would read "C: $\backslash$ Program Files $\backslash I t a s c a \backslash 3 d e c 410 \backslash$ exe64 $\backslash$

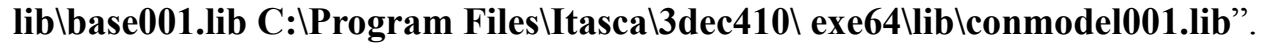

To create a release version, make the same property changes in the "Release" configuration properties, but do not add the "MODELDEBUG" preprocessor definition. Also, remove the "d" suffix from the model name.

Then within 3DEC software, the CONFIG cppudm command will allow the code to accept DLL models. Model DLL files can then load into 3DEC while it is running by giving the command ZONE load filename, where filename is the name of the DLL. DLL files automatically load if they are placed in the

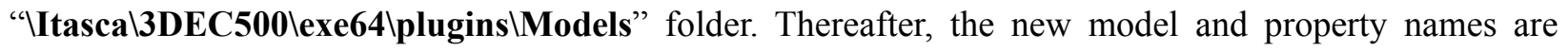
recognizable by $3 \mathrm{DEC}$ and FISH functions that refer to the model and its properties.

\subsubsection{Programming of the new model}

According to the development of the new model in Section 6.1 and the user-defined model in 3DEC in Section 6.2, the viscoelastic-strain-softening model was programmed into 3DEC as a user-defined model. After coding and debugging, the next step is to generate a DLL file named modelvess005_64.dll and copy it to the "Itascal3DEC500 $\backslash$ exe64 $\backslash$ plugins $\backslash$ Models" folder. When the model is assigned to a material, it loads automatically. 
The following section explains the use of the new model in 3DEC software as follows. First, in order to use the user-defined viscous model, the beginning of the commands should give the CONFIG creep cppudm command so that 3DEC software allows the acceptance of user-defined model and creep simulation. In addition, the name of the model is vess (viscoelastic-strain-softening) when programming. The ZONE model vess $<$ range ..... > command assigns the new model to the material of a specific range. Furthermore, one can assign the property values for the new model to the material by the property name and property value following ZONE command. For example, command ZONE bulk value1 mshear value 2 assigns the values of bulk modulus and Maxwell shear modulus to the material.

Aside from the common property in 3DEC, such as bulk modulus, cohesion, friction table, and Kelvin viscosity (shear modulus), the viscous parameters determined from the post-failure region should be assigned to account for the variation in the time-dependent property. The property names are postksh, postkvis and postmvis for Kelvin shear modulus, Kelvin viscosity and Maxwell viscosity in the post-failure region, respectively. If the viscous parameters for failed rock are unassigned, it instead uses the values for intact rock and, as a result, does not consider the variation in the time-dependent property. It is also notable that different tables are necessary for strain-softening behavior and strength degradation. The cohesion table controls strain-softening behavior by showing the relation between cohesion values and effective shear plastic strain, while the friction table controls the strength degradation induced by viscous behavior by showing the relation between friction angle and effective viscous strain. Caution is necessary when assigning these parameters; if leaving these tables unassigned, the material does not show the corresponding behaviors.

\subsection{MODEL PARAMETER DETERMINATION AND CALIBRATION}

\subsubsection{Determining model parameters}

This section describes the proposed laboratory test plan to determine the parameters for the newly developed model. The test plan includes the test to determine the time-independent parameters, the test to determine the variation in the time-dependent property, and the test to describe the strength degradation behavior.

For one type of rock, conducting a series of unconfined and tri-axial compression test is the first step to investigate the time-independent mechanical parameters. At least two confining stresses should be adopted for the tri-axial compression test and at least three specimens for each of the (unconfined and tri-axial) compression test. These tests can determine the mechanical parameters-Young's modulus, poison ratio, and strength parameters (cohesion and friction angle). Also, these tests can study the relation between strength (cohesion) and shear plastic strain controlling the strain-softening behavior in the post-failure region.

In addition, conducting three unconfined compression tests with five relaxation tests, two in the prefailure region and three in the post-failure region, for each specimen can determine the viscous parameters. The two relaxation tests in the pre-failure region can assist in determining the viscous parameters for intact rock, while three relaxation tests conducted in the post-failure region can determine the viscous parameters of failed rock. 
Finally, a series of unconfined creep tests at different stress levels- $80 \%, 85 \%, 90 \%$ and $95 \%$ of UCScan assist in studying the relation between strength values and effective viscous strains. It is important to note that the creep tests should continue until the specimens fail, and a long time is necessary for the creep test if the applied stress is low. One can then calculate the accumulated effective viscous strain from the creep curves, and the strength value corresponds to the applied stress value during the creep test. As a result, a series of creep to failure test can help obtain the strength value associated with a specific effective viscous strain value.

\subsubsection{Calibration for strain-softening behavior}

It is well-known that the strain-softening model should be calibrated for simulations. The typical failure mode for compression test on rock specimens is shear failure: the deformation field localizes into shear bands, and the energy releases and dissipates with band formation. However, the development of shear bands begins due to very small local vibrations in the initial condition and the thickness of the shear band depends on the internal characteristic length associated with the material, such as average length of imperfections or grain diameters.

The 3DEC software can simulate the initiation and evolution of shear bands, as seen in the previous simulations. However, there is no internal characteristic length associated with the material included in the code, which determines the thickness of the shear band. As a result, the thickness of the bands in 3DEC reduces to the smallest dimension resolved by the mesh, which is the zone size. If the band is parallel to the mesh, the typical band thickness is about one zone-width. The band thickness is about three zone-width if the band cuts the grid at an arbitrary angle. Therefore, the solution algorithm in 3DEC reproduces the physics of band formation accurately, but the thickness of the bands and their spacing are strongly dependent on the mesh.

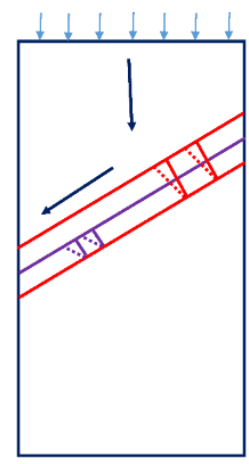

Fig. 6.5. Model to consider strain softening behavior with different failure band thicknesses

According to the strain-softening memo from Itasca, the strain softening rate highly depends on the mesh. Consider the example of a softening material using a given softening law. 3DEC can simulate a localization test and produce a load-displacement curve measured from the platen. The strain concentrates in a band during strain softening, and the strain within the band is irrecoverable or plastic. For instance, with a given displacement increment, the concentrated strains within the band are different depending on the width of the band, which in turn depends on the zone size. Smaller zones lead to thinner band. For a given displacement increment, the thinner band needs to deform more than that of a thicker band. For a 
given softening law, more plastic deformation within the shear band demonstrates that the strength decreases more rapidly, leading to steeper slope for the stress-strain curve in the post-failure region and resulting in more brittle behavior.

The mesh dependence is a common problem for a standard continuum formulation; as such, correction involves the incorporation of a length scale. The method that can solve this problem includes enrichment of standard formulation with Cosserat continuum, plasticity gradient model and non-local model, and standard method regulation using energy principles. Each method has its own respective advantages and drawbacks. Regulation method is the only method available for 3DEC at this time.

The following is a summary of the recommended calibration and regularization procedure. The results of tri-axial or shear experimental tests can assist in calibrating the numerical softening rate. The procedure consists of performing a numerical simulation to reproduce the lab test, using a given grid size. In the numerical experiment, the numerical band softening rate adjusts to fit the lab test response as close as possible. If using the same zone size for the problem simulation, no regulation is necessary. If increasing the zone size, the numerical softening rate increases in proportion and vice-versa.

\subsubsection{Calibration for viscous behavior}

Table 6.1 Parameters for model calibration and verification

\begin{tabular}{|c|c|c|c|}
\hline \multicolumn{3}{|c|}{ Parameter } & Value \\
\hline \multirow{6}{*}{ Time-Independent } & \multicolumn{2}{|c|}{ Density (Kg/m3) } & 2563 \\
\hline & \multicolumn{2}{|c|}{ Bulk Modulus (GPa) } & 40.17 \\
\hline & \multicolumn{2}{|c|}{ Shear Modulus/Mshear (GPa) } & 26.46 \\
\hline & \multicolumn{2}{|c|}{ Cohesion (MPa) } & 8.34 \\
\hline & \multicolumn{2}{|c|}{ Friction Angle (degree) } & 30.00 \\
\hline & \multicolumn{2}{|c|}{ Tensile Strength (MPa) } & 6.89 \\
\hline \multirow{6}{*}{ Time-Dependent } & \multirow{3}{*}{ Pre-Failure } & Mviscosity (GPa*s) & $2.74 \mathrm{E}+06$ \\
\hline & & Kshear (GPa) & $4.51 \mathrm{E}+02$ \\
\hline & & Kviscosity $\left(\mathrm{GPa}{ }^{*} \mathrm{~s}\right)$ & $9.92 \mathrm{E}+04$ \\
\hline & \multirow{3}{*}{ Post-Failure } & Mviscosity (GPa*s) & $2.74 \mathrm{E}+05$ \\
\hline & & Kshear (GPa) & $9.03 \mathrm{E}+01$ \\
\hline & & Kviscosity $\left(\mathrm{GPa}^{\prime} \mathbf{s}\right)$ & $1.24 \mathrm{E}+03$ \\
\hline
\end{tabular}

Mesh dependence is an important factor for the viscous-strain-softening model, which is a combination of viscous model and strain-softening model. The previous section has discussed the mesh dependence for strain softening model. But what about the viscous behaviors in the post-failure region? Is there mesh dependence as well? One notable feature of the viscous-strain-softening model is that it takes into account the variation in the time-dependent property of rocks before and after failure. If there is a failure band in a rock specimen, the stress relaxation rate will be different inside and outside the band. The stress relaxation rate inside the band is the same regardless of the band thickness; as a result, the overall response of the specimens with failure band of different thicknesses are different. In addition, if there is a failure zone within the roof, the thickness or size of the failure zone varies with zones of different sizes. The influence of relaxation or creep within this area on the surrounding areas may be different. Therefore, it is necessary 
to investigate the mesh dependence of viscous behaviors in the post-failure region.

First, the study focused on the mesh dependence of viscous behaviors in the post-failure region from relaxation point of view. In the simulation of unconfined compression test on a rock specimen, all the elements fail at the same time. If the vess model describes the rock behavior, the post-failure viscous parameters activate at the moment when failure occurs. The result is that all the zones assigned with postfailure viscus parameters fill the whole specimen, rather than being located solely within a band. Therefore, it is impossible to load the specimen to failure and then perform a relaxation test with the purpose of investigating the mesh dependence of viscous behaviors. Instead, this study used a different methodology.

Fig. 6.6 shows the use of bands with different thicknesses ( 0.3 inch and 0.6 inch, respectively) to represent the failure area, similar to the shear bands. Within these bands the tests assigned the viscous parameters with the values from the post-failure region and assigned with the viscous parameters of the pre-failure region to the other areas. During the test, velocity first applied to the top of the specimen to load the specimens to the same stress level; then, fixing the top of the specimen initiated a relaxation test. The relaxation curves can help study the influence of band thickness on the time-dependent behaviors.

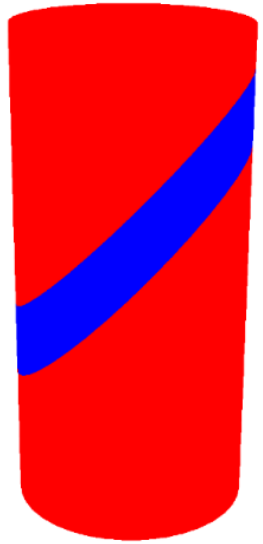

(a) Thick band

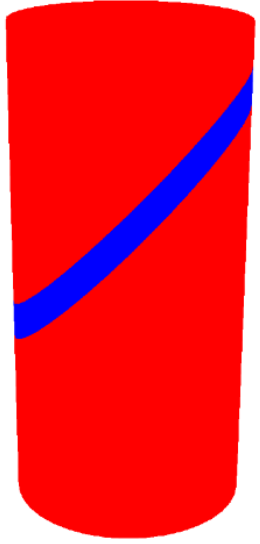

(b) Thin band

Fig. 6.6. Plot of property distribution within specimens

The simulation results with different band thicknesses show differences in the relaxation behavior. From the stress-strain curve in Fig. 6.7, it is notable that the stress-strain curves overlap before the relaxation test with only minor difference in the relaxation behavior. A smaller amount of stress relaxed from the specimen with thin band; the relaxation curve in Fig. 6.8 further confirmed this, where the relaxation curve for the specimen with thin band is below that of the specimen with thick band. These results imply that slightly larger amount of stress relaxed from the specimen with thick band. Therefore, the thickness of the band can affect the stress relaxation rate, but the influence is minor. 


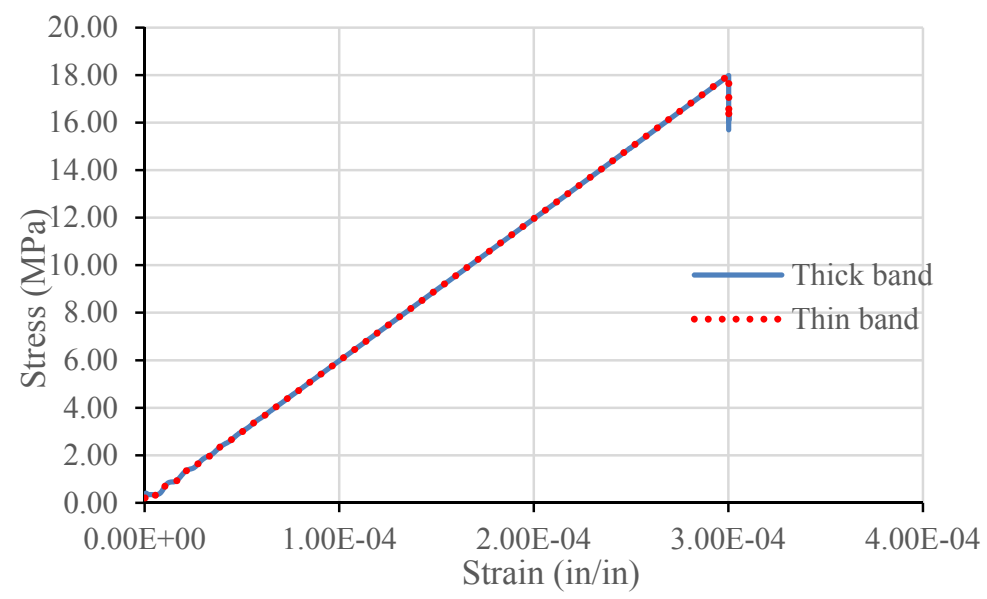

Fig. 6.7. Stress-strain curves for the specimens with bands of different thicknesses

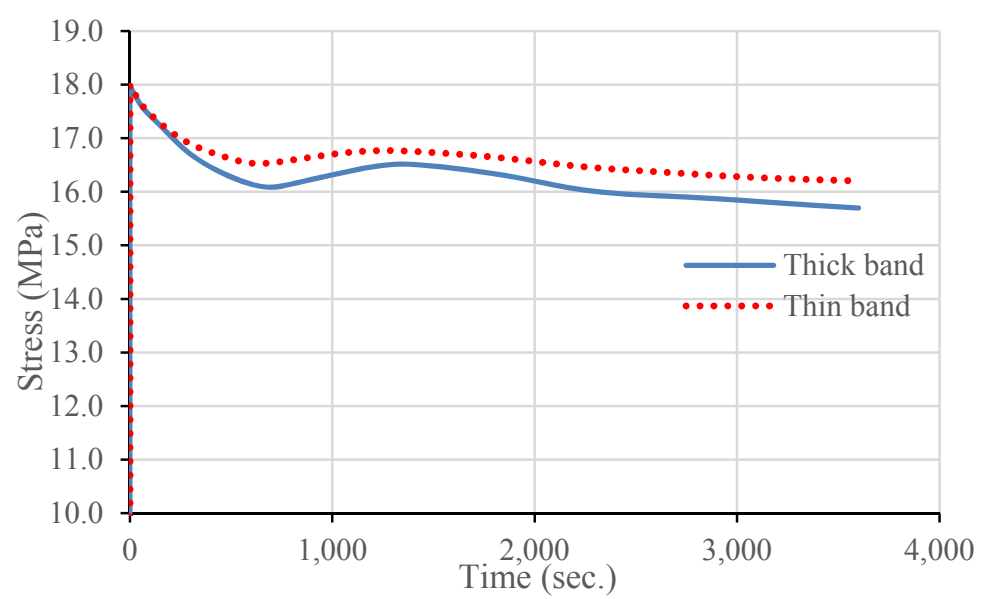

Fig. 6.8. Stress-time curves for the specimens with bands of different thicknesses

A procedure similar to that for strain softening model, was tried to calibrate the time-dependent behaviors of the viscoelastic-strain-softening model. The thickness of the thick band was two times as that of the thin band in the above simulations; as such, different stress relaxation behaviors occurred. In order to calibrate the model, all the viscous parameters for the model with thick band increased two times their previous values and the tests ran again. Figs. 6.9 and 6.10 show the simulation results. These figures show that the stress-strain curves and relaxation curves all overlap, indicating that increasing the viscous parameters proportionally when the element size increases can calibrate the viscous parameters in the postfailure region. 


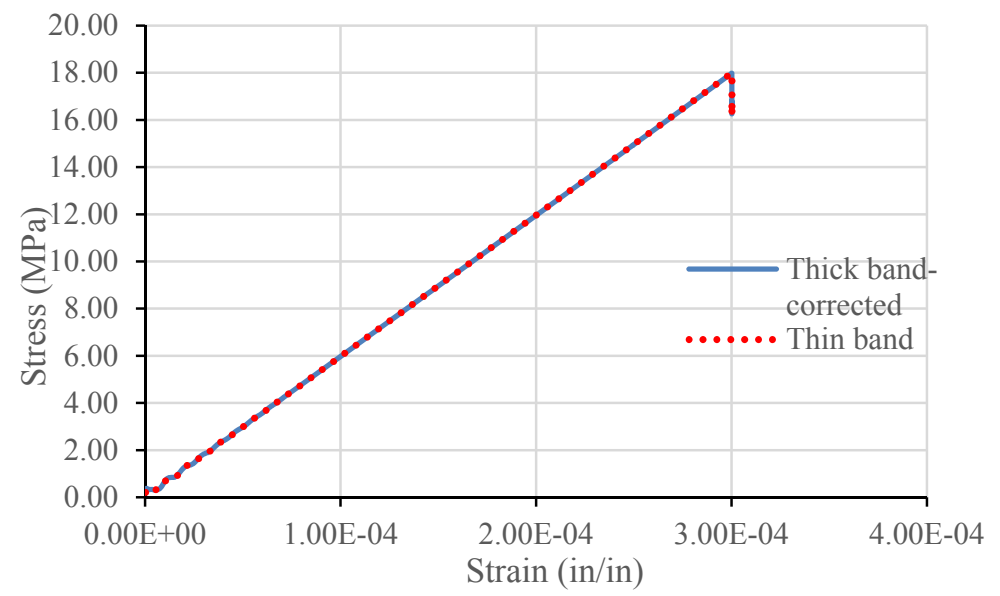

Fig. 6.9. Stress-strain curves for the specimens with bands of different thicknesses after correction

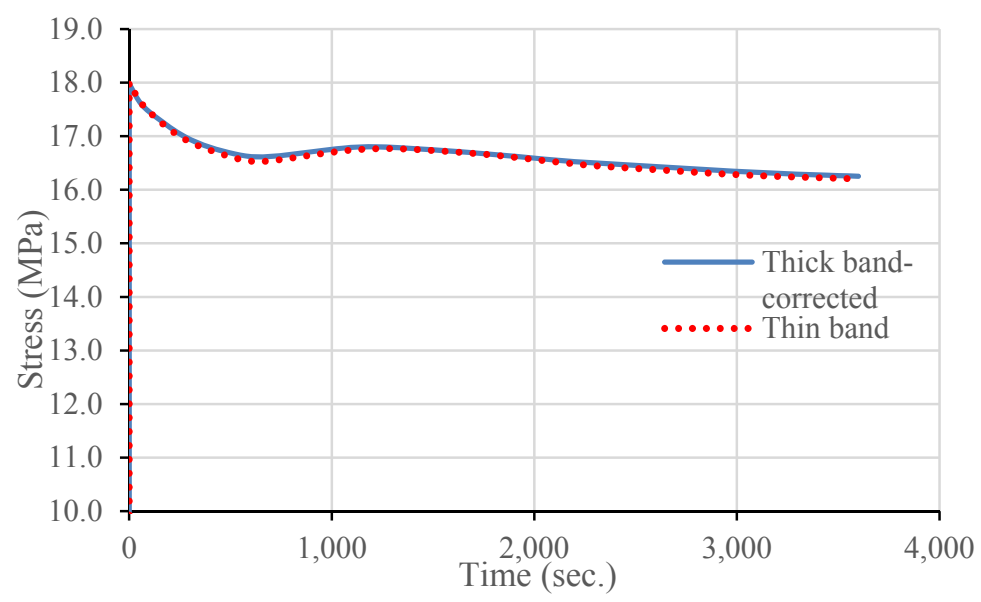

Fig. 6.10. Stress-time curves for the specimens with bands of different thicknesses after correction

\subsection{MODEL VERIFICATION}

This study conducted a series of numerical simulations on simulated rock specimens to verify the generated vess model. Table 6.1 presents the time-independent and time-dependent parameters. Figs. 6.11 and 6.12 show the decay of cohesion and friction angle with shear plastic strain and effective viscous strain.

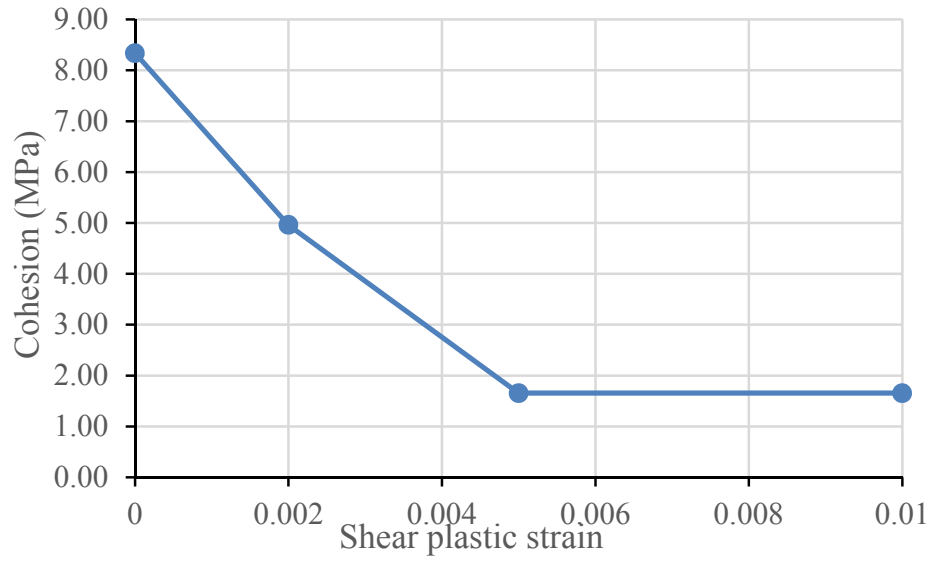

Fig. 6.11. Variation of cohesion with shear plastic strain 


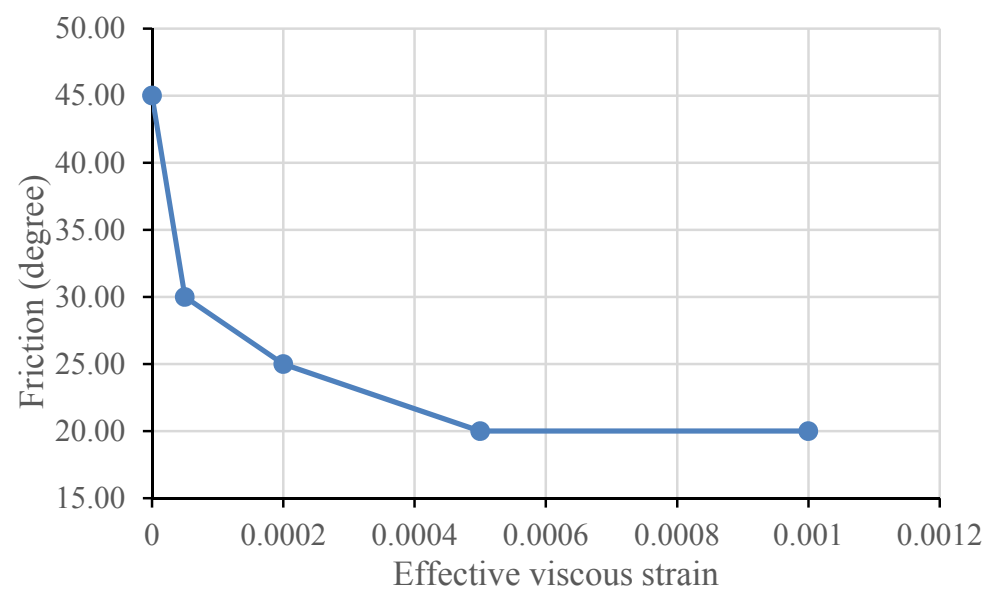

Fig. 6.12. Variation of friction angle with effective viscous strain

\subsubsection{Numerical simulation of unconfined compression test}

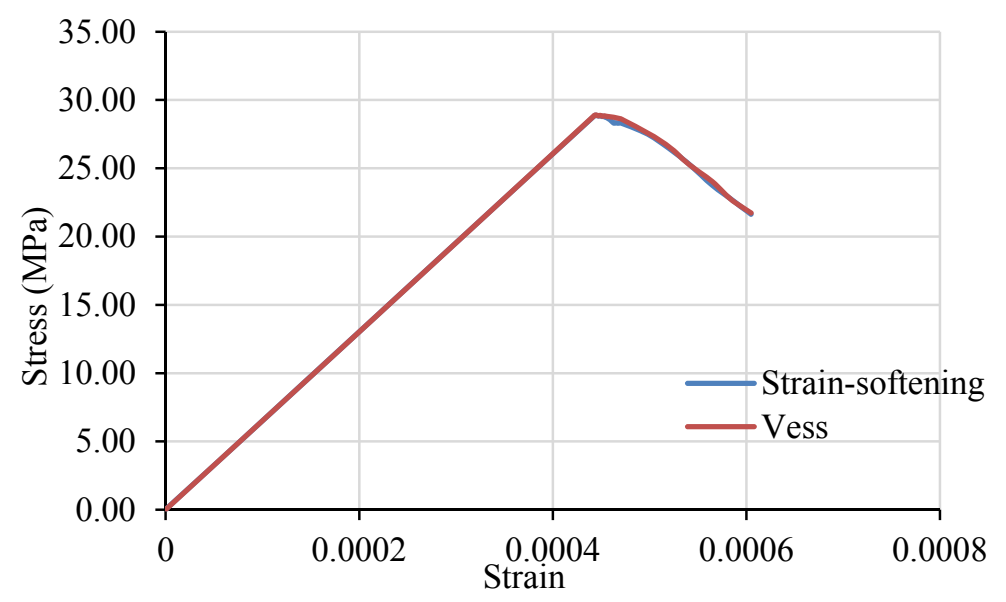

Fig. 6.13. Comparison of strain-softening behaviors between the strain-softening model and vess model

Unconfined compressive tests were simulated first with the strain-softening model and viscoelasticstrain-softening model. Except for the constitutive model, all the other parameters and model configurations remained the same. Fig. 6.13 shows the resulted stress-strain curves. The stress-strain curves from the two different models show good agreement, except that the stress-strain curve for the viscoelastic-strainsoftening model is smoother than the one for the strain-softening model. This demonstrates that the viscoelastic-strain-softening model can represent the post-failure behavior described by the strain-softening model.

\subsubsection{Unconfined compression test with relaxation tests}

Next, unconfined compression test with two relaxation tests were simulated with two different models to verify the new model. The tests assigned Burgers model with Mohr-Coulomb failure criterion, referred as the 'cvis' model in 3DEC, to one specimen. For comparison, the tests assigned the viscoelastic-strainsoftening model without strain-softening behavior to the other specimen. As a result, the two models show perfect-plastic behavior after failure. Under this circumstance, the difference between these two models is 
that the viscoelastic-strain-softening model considers the variation in the time-dependent property before and after failure. The first relaxation test performed in the pre-failure region, and the other in the postfailure region. Therefore, the relaxation test results in the pre-failure region are comparable between the viscoelastic-strain-softening model and Burgers model; the relaxation test results in the post-failure region, in turn, can verify the capability of the new model in considering the variation in the time-dependent property.

The compression test was considered to be time-independent, regardless of the influence of time or strain rate. The tests applied a constant velocity boundary on the top of the simulated specimen, fixing the top of the specimen upon reaching a determined timestep. The time-dependent simulation then activated to simulate a relaxation test. After the relaxation test, the tests applied the velocity boundary again to load the specimen to the post-failure region for the second relaxation test.

Figs. 6.14 and 6.15 show the simulation results in the form of stress-strain curves and relaxation curves. With two different models, the stress-strain curves before the second relaxation test and the relaxation curves for the first relaxation test show good agreement. This agreement indicates that the viscoelasticstrain-softening model can accurately reproduce the behaviors described by Burgers model with MohrCoulomb failure criterion when not considering strength degradation and strain-softening behavior. In addition, there is significant difference in the second relaxation test between these two models. The viscoelastic-strain-softening model considers the variation in the time-dependent property by changing the time-dependent parameters when failure occurs. The variation results in different relaxation behaviors in the post-failure region. For these two models, the difference in the relaxation curves for the relaxation tests in the post-failure region demonstrates that the new model can consider the variation in the time-dependent property of rocks.

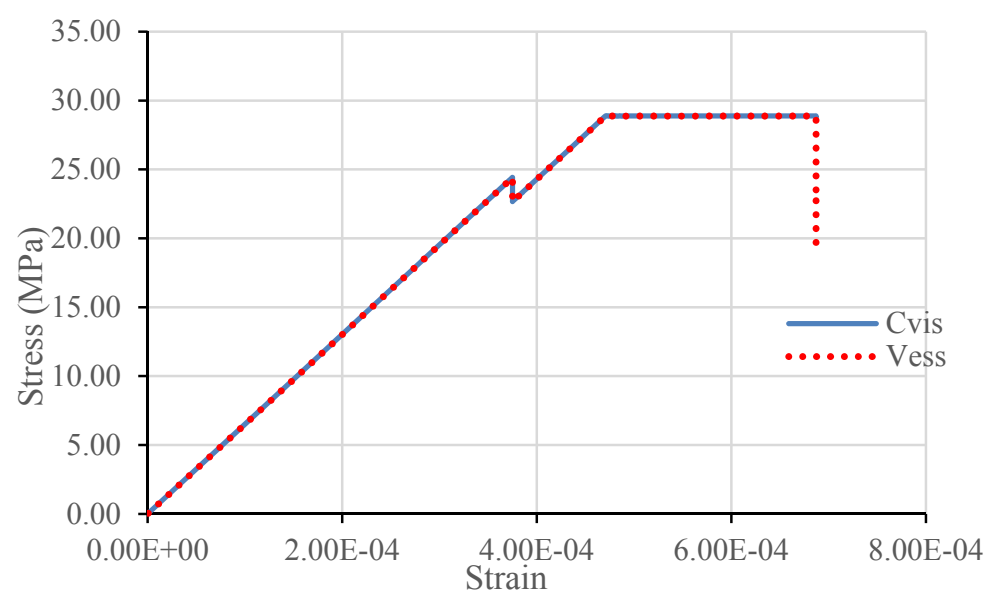

Fig. 6.14. Stress-strain curves with cvis and vess model with perfect plasticity 


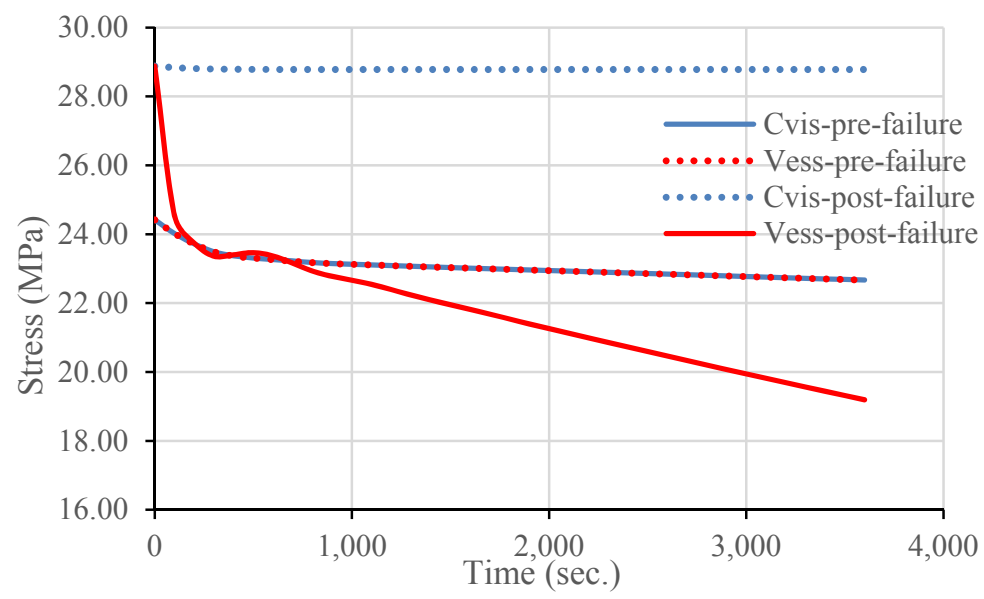

Fig. 6.15. Relaxation curves with cvis and vess model with perfect plasticity

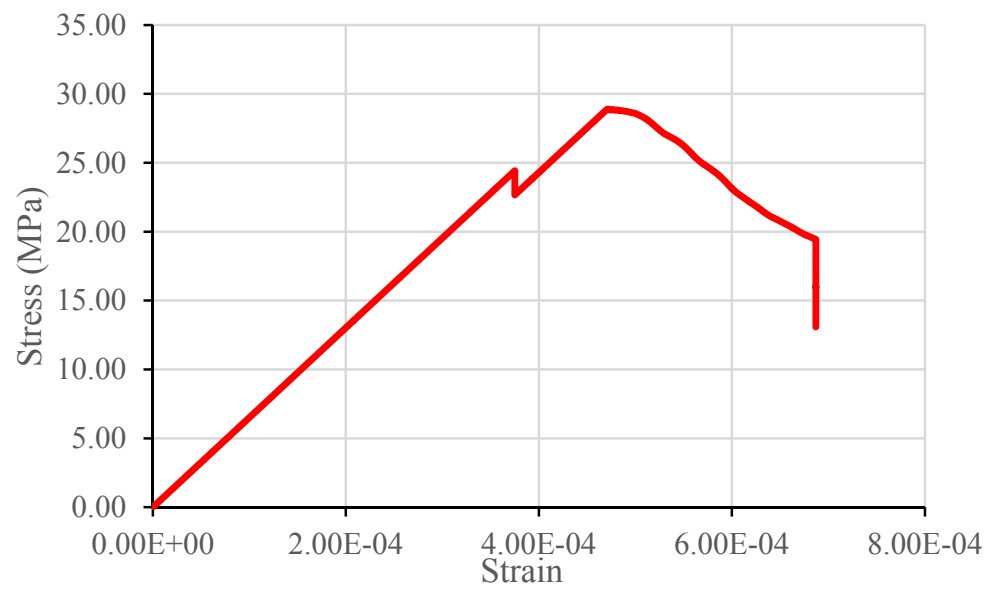

Fig. 6.16. Stress-strain curve with vess model considering strain-softening behavior and variation in the timedependent property

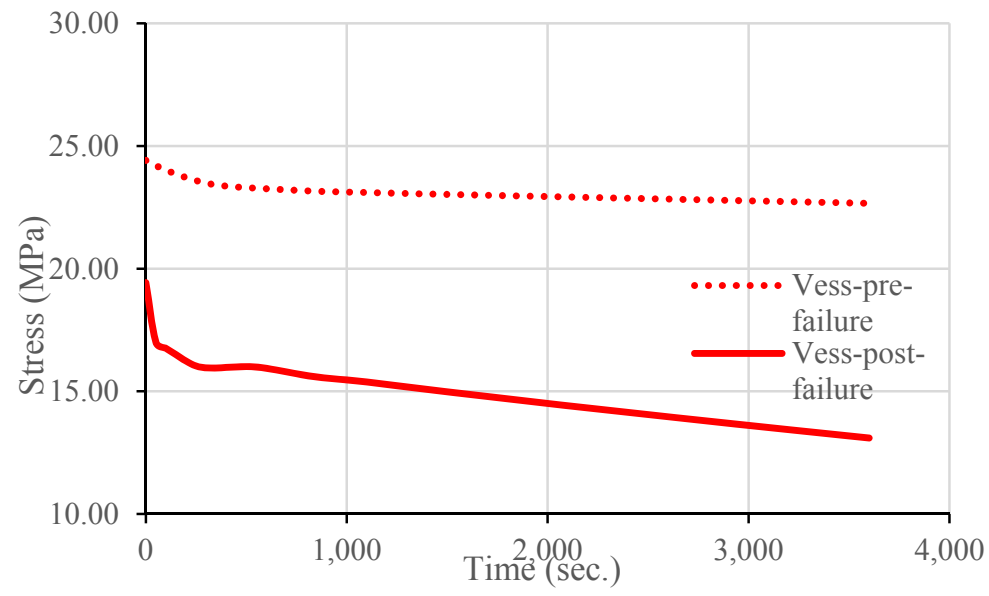

Fig. 6.17. Relaxation curves with vess model considering strain-softening behavior and variation in the timedependent property 
Furthermore, the unconfined compression with two relaxation tests was simulated with the viscoelasticstrain-softening model, whereas the second relaxation test ran during the strain-softening process. Figs. 6.16 and 6.17 show the simulation results. The model can describe the strain-softening behavior and the viscous behaviors observed in laboratory test. Although the starting stress for the second relaxation test is lower than that of the first, by considering the variation in the time-dependent property, more stress relaxed from the test conducted in the post-failure region for the same duration.

\subsubsection{Creep failure}

This study simulated a series of unconfined creep tests to verify the new model in reproducing the creep failure behavior. Fig. 6.12 shows the strength degradation relation given by the relation between friction angle and the effective viscous strain, which one can input into 3DEC code as a table. Fig. 6.18 shows the resulting strength degradation relation. The effective viscous strain gradually accumulates with time during a creep test, leading to a decreasing strength value. Specimen failure occurs when the strength value reduces to the value of the applied constant stress.

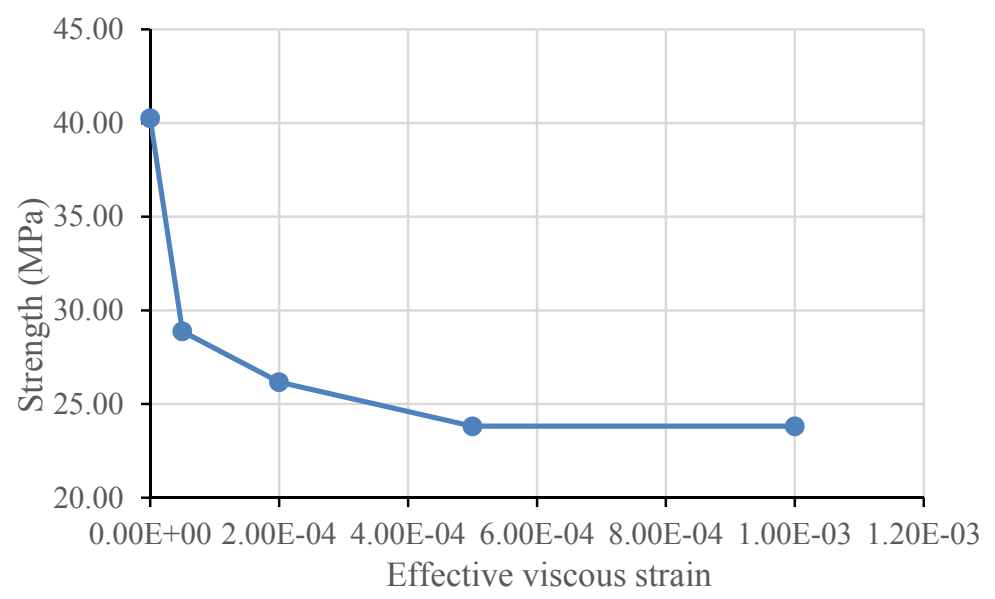

Fig. 6.18. Strength degradation relation

The simulation results demonstrate that the model can reproduce the creep failure of laboratory specimens. Fig. 6.19 illustrates that the simulated specimens show typical creep behavior, where the creep curves have three stages: primary, secondary, and tertiary. The accelerating strain rate after the secondary stage indicates the failure of the specimen; therefore, when the creep test starts, the simulated specimens fail over certain amount of time. However, there are three specimens continuing to deform without failure; this is due to the provided strength degradation relation. As shown in Fig. 6.18, the strength degradation relation becomes a horizontal line when the strength reduces to $23.81 \mathrm{MPa}$, indicating that this is the lower bound for strength degradation. If the applied stress is below this value, the specimen continues deforming without failure. In addition, Fig. 6.19 shows that the time to failure depends on the applied constant stress. It takes longer time to fail the specimen if the applied constant stress is lower. Fig. 6.20 plots the relation between time to failure and the applied stress, showing similar trend as the strength degradation relation in Fig. 6.18. 


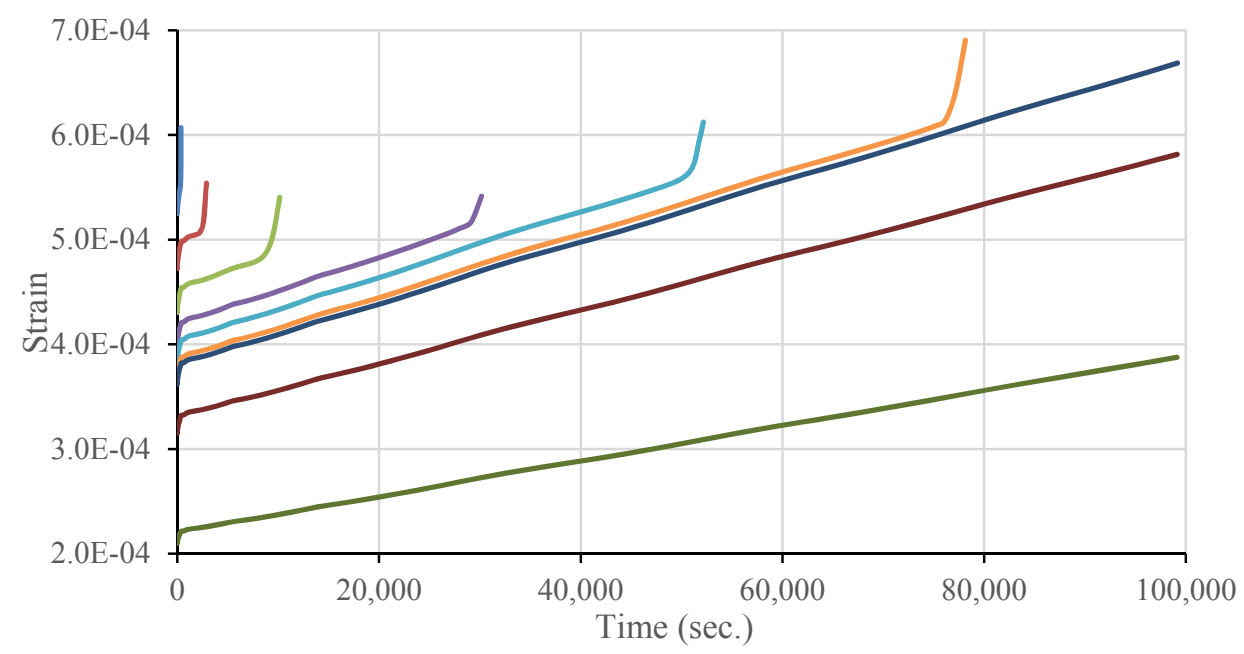

Fig. 6.19. Results of simulated creep tests with strength degradation

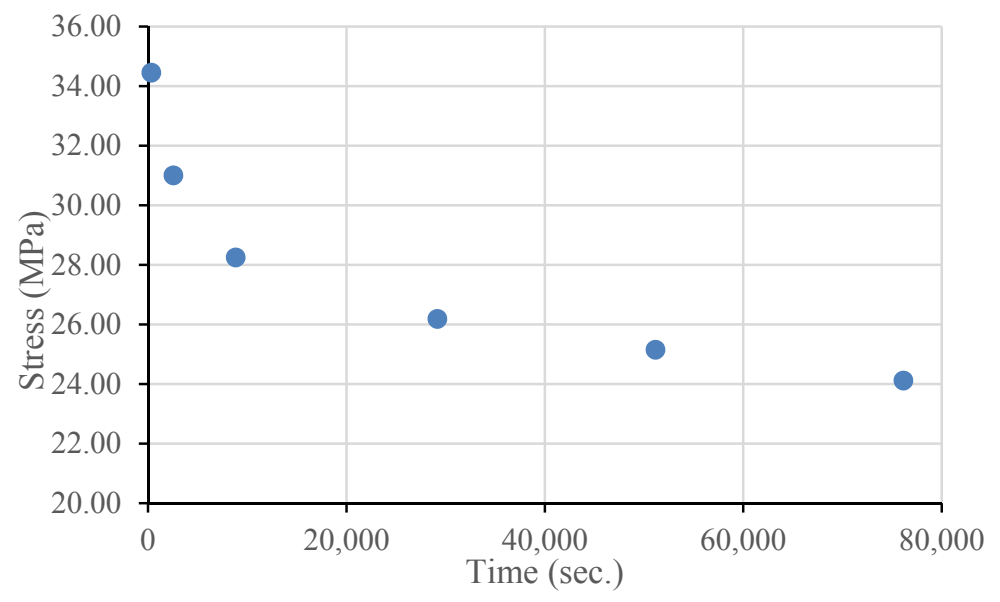

Fig. 6.20. Time to failure with different constant stresses

\subsection{SUMMARY}

The formation of roof fall in underground coal mines is a time-dependent process. Time-dependent roof failure is the result of the progressive propagation of localized failure and involves various behaviors, including time-dependent behavior of intact and failed rock, strain-softening behavior, and strength degradation caused by viscous behaviors. Various laboratory tests have observed these behaviors; however, there is no current model that represents all of these important behaviors.

This chapter proposed a new viscoelastic-strain-softening model to describe the time-dependent and time-independent behaviors of rocks in numerical simulation. In this model, Burgers model represents the viscoelastic behavior of rocks, regardless of whether the rock is intact or failed. The model represents the difference in the viscous property between intact and failed rock by varying viscous parameters when failure occurs. Using different viscous parameters for rocks before and after failure accounts for the variation in the time-dependent property of rock. In addition, the strain-softening model describes the behavior of rock in the post-failure region, where the strength decreases with increasing effective plastic strain. The user can input the relation between cohesion and effective plastic strain to control the strain-softening behavior. A 
strength degradation relationship, similar to the strain-softening model, describes the relationship between rock strength and effective viscous strain to account for the strength deterioration caused by viscous behaviors. The relationship between friction angle and effective viscous, input by the user, controls the strength degradation. Finally, this study programmed the proposed model with $\mathrm{C}++$ and compiled it as a user-defined model in 3DEC in the form of a DLL file. The model can then assist in performing numerical simulation. This chapter also explained the procedures to obtain associated parameters and to calibrate the model.

Finally, this study simulated a series of laboratory tests to verify the developed model. The simulation of unconfined compression tests with the strain-softening model and the new model shows good agreement, demonstrating that the new model can describe strain-softening behavior. The simulated unconfined compression tests with two relaxation tests also demonstrate that the new model can reproduce the behaviors described by the built-in cvis model in 3DEC. The new model can further describe strainsoftening behavior and the variation in the time-dependent property for rocks before and after failure. The time to failure in the simulated rock failure under unconfined creep condition also shows good agreement with the strength degradation relationship. In summary, the simulation results demonstrate that the new model can reproduce the time-dependent strain-softening behaviors observed during various laboratory tests. 


\section{CHAPTER 7 \\ NUMERICAL STUDY ON THE TIME-DEPENDENT BEHAVIOR OF MASSIVE ROOF FOR UNDERGROUND OPENINGS}

\subsection{INTRODUCTION}

Field observations have demonstrated that the formation of roof falls is a process involving timeindependent and/or time-dependent behavior. The time-independent behavior mainly refers to the response of mine roof to the stress redistribution induced by mining activities. The adjacent mining activities, like the development of adjoining entries, mining cutting sequences and the movement of longwall face, and the failure propagation within the roof can both affect the local stress condition of the mine roof. Numerical simulations usually ignore the influence of time for these situations. In addition, the time-dependent behavior includes the time-dependent deformation of roof rock, the rock deterioration, and the timedependent failure of the roof rock. The stress within the roof redistributes along with the time-dependent failure development. As a result, the time-independent and time-dependent behavior interacts with each other.

The common simulation procedure for creep simulation of underground structures involves two steps. The first step is to run the model to equilibrium after excavation. The objective is to determine the timeindependent response of the surrounding rocks resulted from the excavation-induced stress redistribution. The unbalance force or deviatoric stress due to the excavation reduces as well to keep the numerical stability for the creep simulation. Afterwards, the second step is to start the creep simulation by assigning a creep timestep; this allows for the investigation of the time-dependent response of the mine roof. Therefore, the creep simulation will investigate the time-independent response of the mine roof to excavation as well as the time-dependent response.

However, this process can be affected by various factors. Primarily, the mechanical behavior and property of the roof rock is one key factor affecting the roof stability in underground mines. These are represented by the constitutive model and associated parameters in numerical simulations. Chapter 6 detailed the proposing and programming of Burgers model incorporating with the variation of the timedependent property, the strength degradation, and strain-softening behavior into 3DEC as a user-defined model; Chapter 3 and Chapter 5 showed the laboratory tests to determine to mechanical properties of coal measure rocks. This chapter used the new model vess to represent the time-independent and time-dependent behaviors of the mine roof rocks and used the calibrated rock properties from the laboratory tests in Chapter 3 and Chapter 5.

Aside from the aforementioned behaviors, other factors also affect the time-dependent roof stability. The time-dependent behavior or the development of the viscous deformation of rock is highly dependent on the stress conditions. Any factor affecting the stress distribution within the mine roof will affect the timedependent deformation and failure of roof rock, further affecting the roof stability. The in-situ stress and stress path are two key factors affecting the roof stability (Ray 2009). The directional horizontal stress 
affects the roof stability and it is necessary to conduct numerical simulation to determine its influence from a time-dependent point of view (Gadde and Peng 2004). In addition, the development of an entry is not achieved in one step, as it takes time to develop an entry. During the entry development, one point within the mine roof advancing the mining face may experience an increase in stress when the mining face moves closer and experience a gradual release of confinement after the mining face passes the point. A realistic way to simulate the time-dependent development of an entry is to divide the excavation into many steps and introduce time into the numerical simulations between mining steps. In this way, one can capture the influence of the stress path that the roof rock may experience.

In summary, this chapter will study the time-dependent response of the massive mine roof numerically. It will numerically simulate a mine entry with a specific in-situ stress condition to investigate the process of time-dependent deformation and failure of the mine roof; in turn, this will determine the influence of the time-dependent behavior and property of the roof rock on the roof behavior. In addition, the numerical simulations will use different horizontal stresses and step-wise excavation. The comparison of the timedependent roof behavior between these different situations will likely demonstrate the influence of the directional horizontal stress and the step-wise excavation on the time-dependent response of the mine roof.

\subsection{STUDY ON THE TIME-DEPENDENT BEHAVIOR OF MASSIVE MINE ROOF}

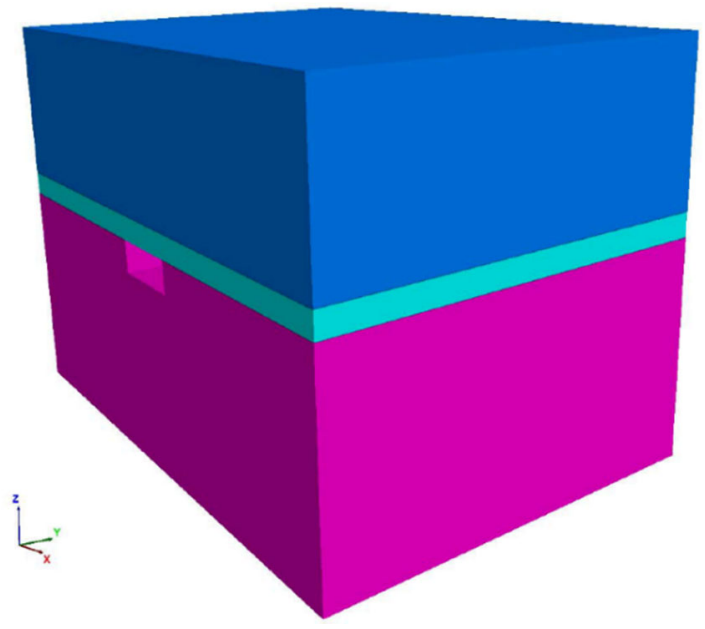

Fig. 7.1. Mine model with massive roof

The shale roof rock is normally laminated or bedded in Pittsburgh coal seam. However, for simplicity, the shale roof is simulated as massive roof in the numerical simulations with finite differential codes and the influence of lamination and bedded plane is taken into account by reducing the rock strength and deformation modulus. In this study, one mine model with one entry was generated to study the timedependent behavior of mine roof. The model ran to equilibrium condition to obtain the geostatic state before mining. The entry was excavated, obtaining the failure condition and stress distribution after the excavation by running the model to equilibrium. Creep runs then commenced to simulate the time-dependent deformation, rock property deterioration, failure propagation and time-dependent stress redistribution. The simulation process shows that one can consider the failure and stress state at equilibrium to be the initial 
state of the following creep simulation, which affects the creep simulations. Therefore, this study examined the state at equilibrium first.

The following figures present the distribution of plastic state, rock property and stress distribution at equilibrium. The distribution of major principal stress, shown in Fig. 7.2, shows that there is an arch-shaped band with high major principal stress. The arch starts from the two corners above the mine entry and extends from the shale immediate roof into the above sandstone roof. The thickness of the arch-shaped band on the right side is much thicker than that on the left side. This difference indicates that, due to the directional horizontal stress, the stress concentration on the right side is more significant than that on the left side. In addition, corresponding to the stress distribution, more failure occurs above the right corner than above the left corner within the immediate roof, as shown in Fig. 7.3. Due to the high stress concentration above the mine entry corners, the failure initiates from these locations. The plot of cohesion distribution in Fig. 7.4 also shows decay of cohesion within the failed elements, which is the result of accumulated shear plastic strain.

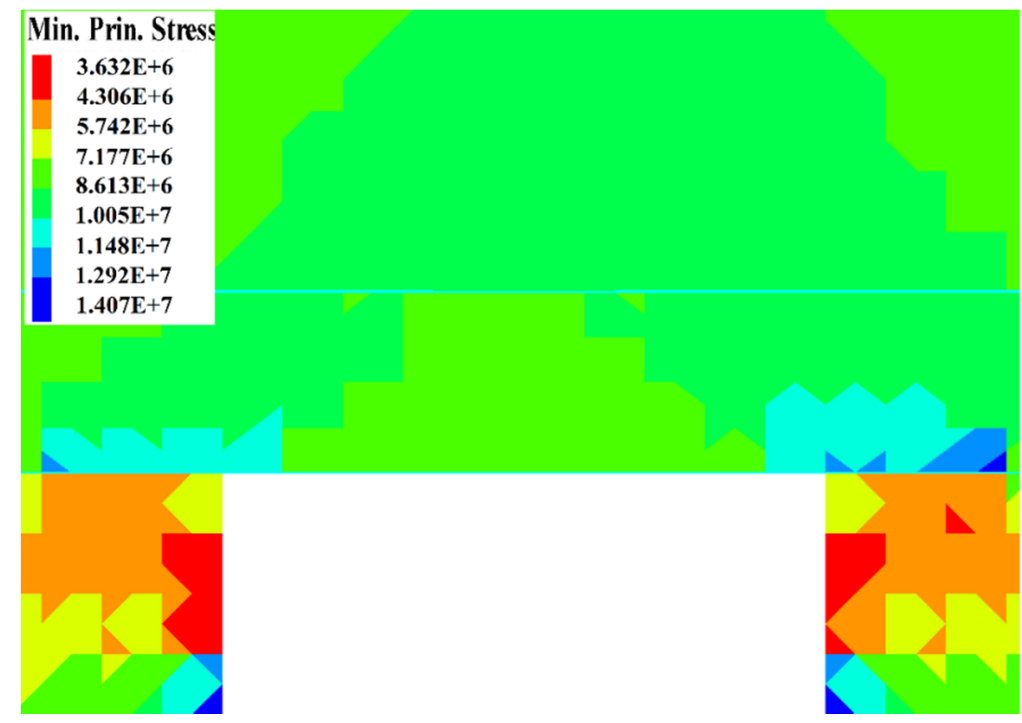

Fig. 7.2. Distribution of major principal stress at equilibrium (unit: $\mathrm{Pa}$ )

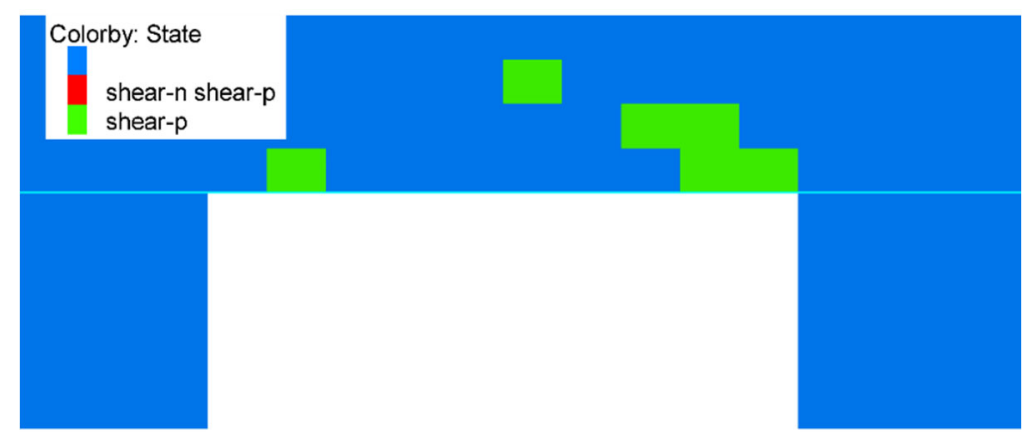

Fig. 7.3. Failure condition at equilibrium 


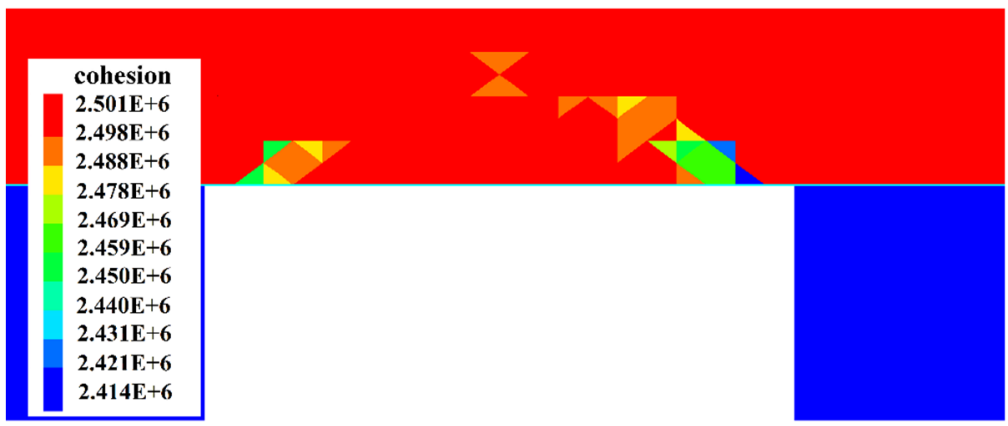

Fig. 7.4. Cohesion distribution at equilibrium (unit: $\mathrm{Pa}$ )

In addition, Fig. 7.5 plots the distribution of deviatoric stress at equilibrium. The rock behaves as elastic under volumetric stress condition and as viscoelastic under deviatoric stress condition; this is due to the constitutive model and means that the distribution of deviatoric stress affects the viscoelastic behavior. As the creep simulation initiates after the model runs to equilibrium, the distribution of deviatoric stress at equilibrium is critical in identifying the locations where the viscous deformation occurs in the creep simulations. Fig. 7.5 also shows that there is an arch-shaped band with high deviatoric stress. However, the distribution of the band is different from the band of high major principal stress. As the deviatoric stress affects the viscous deformation, it is likely that more viscous deformation will accumulate within the archshaped band with high deviatoric stress, further deteriorating the rock property along this band. Fig. 7.6 plots the distribution of friction angle after 30 seconds. The decay of friction angle occurs along the band with high deviatoric stress to coalesce the failures initiating from the two entry corners. It is important to note that there are a few elements without rock deterioration, as these elements already fail at equilibrium and the rock degradation of failed rock is not considered in the new viscoelastic-strain-softening model. As a result, failed elements at equilibrium do not decay with time in creep simulation and maintain the original friction angle.

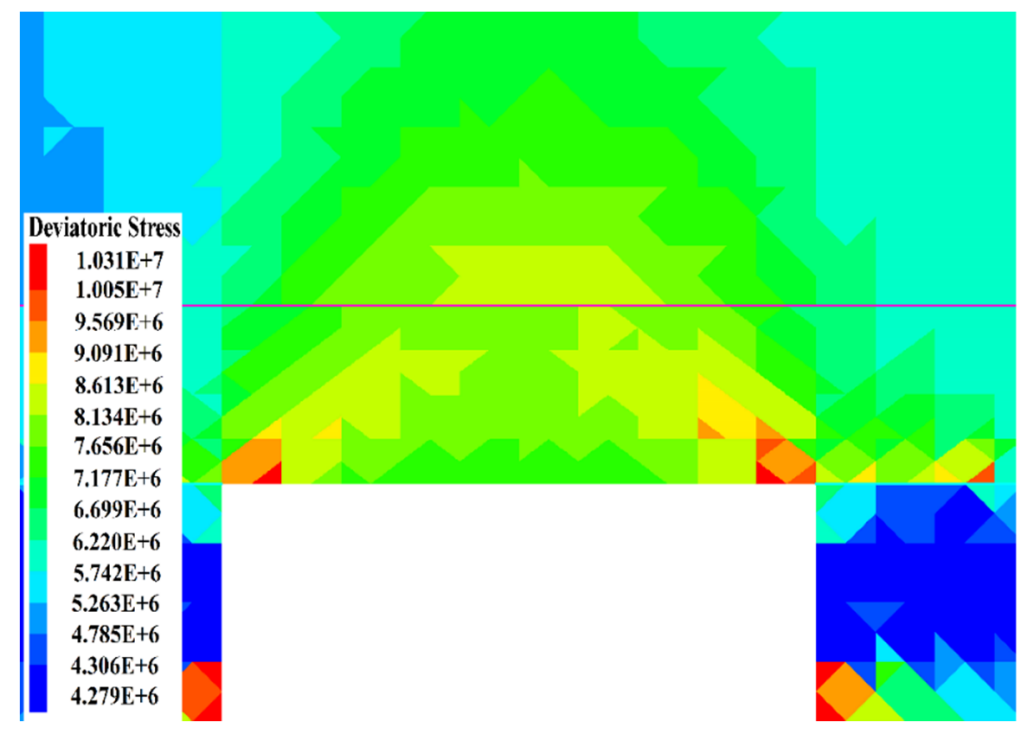

Fig. 7.5. Distribution of deviatoric stress at equilibrium (unit: Pa) 


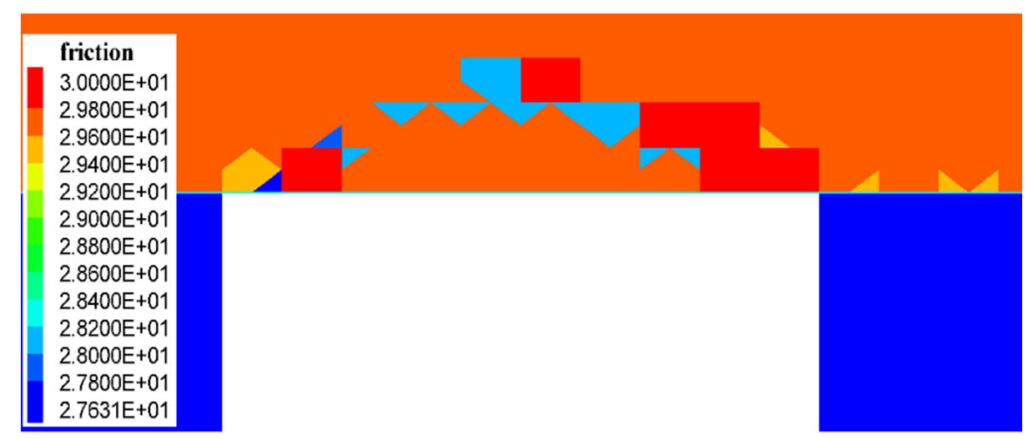

Fig. 7.6. Distribution of friction angle after $30 \mathrm{sec}$. (unit: degree)

The failure propagation in the following few minutes in the simulation was examined. Figs. 7.7, 7.8 and 7.9 plot the failure condition at different time. Comparing the failure condition at equilibrium and after 5 , 6- and 7-minutes reveals that the failures gradually propagated along the arch-shaped band with high deviatoric stress to coalesce the failures initiating from the two entry corners. This coincides with the decay of friction angle, shown in Fig. 7.6. During the creep simulation, relatively more significant viscous deformation occurs within the area with high deviatoric stress than other areas. The direct result is that the rock within these elements deteriorates with time. Along with the strength degradation and increasing stress difference induced by the difference in the viscous property between intact and failed rock, failure occurs within these elements, resulting in the time-dependent failure propagation. As shown in Fig. 7.10, once the elements fail, shear plastic strain accumulated in these elements and further reduced the strength of failed rock by strain softening behavior.

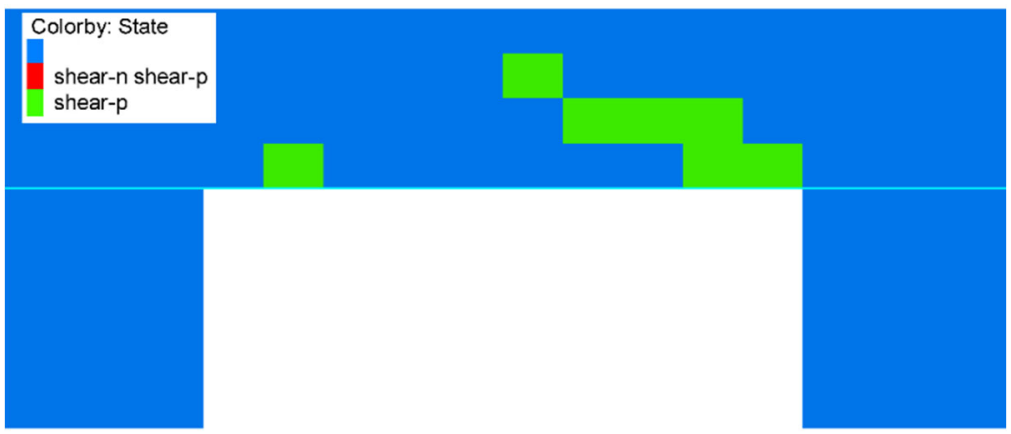

Fig. 7.7. Failure condition after $5 \mathrm{~min}$.

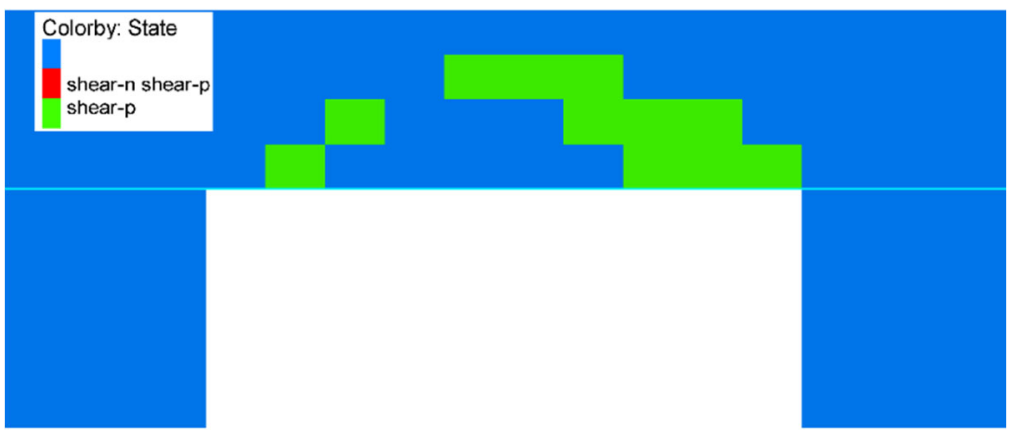

Fig. 7.8. Failure condition after $6 \mathrm{~min}$. 


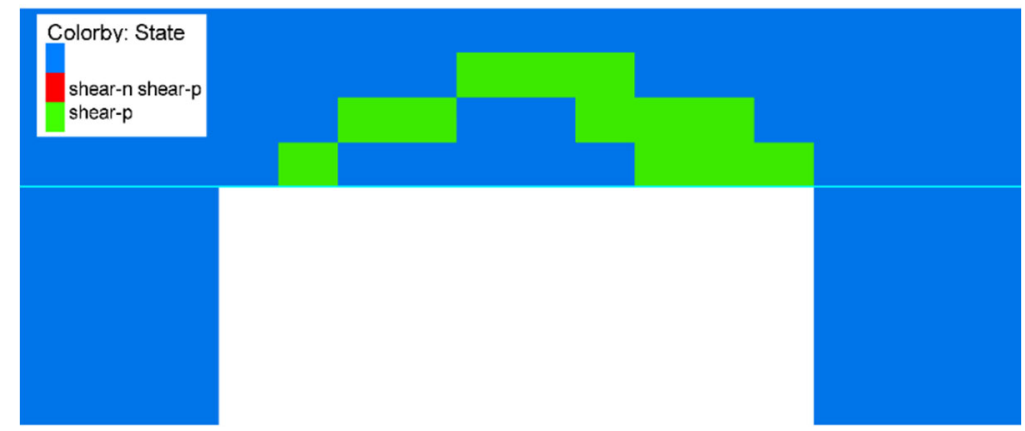

Fig. 7.9. Failure condition after $7 \mathrm{~min}$.

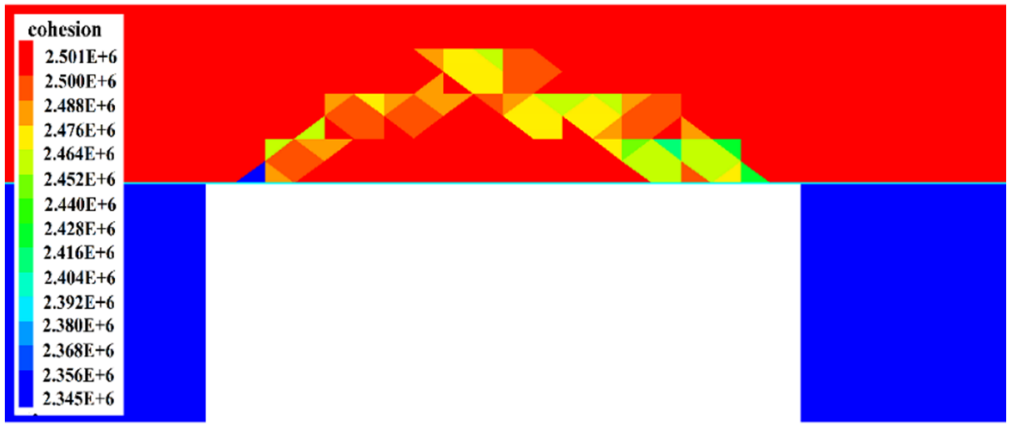

Fig. 7.10. Cohesion distribution after 7 min. (unit: Pa)

Furthermore, Fig. 7.11 plots the distribution of deviatoric stress after 7 minutes. Comparison of Figs. 7.5 and 7.11 shows that, along with the viscous deformation, the stress relaxed from the mine roof, and the area with relatively high deviatoric stress changed with time. Comparing Figs. 7.8 and 7.11 shows that the area with relatively high deviatoric stress transferred out of the failed elements and surrounded the failed elements. This observation indicates that rock deterioration will concentrate in the area surrounding the failed elements and it is highly possible that the failure will continue propagating from these areas over time. Figs. 7.12 and 7.13 plot the failure condition in the following few minutes. Comparison of Figs. 7.9, 7.12 and 7.13 shows that, after the failures coalesced within the immediate roof, the failure gradually propagated horizontally from the failed elements.

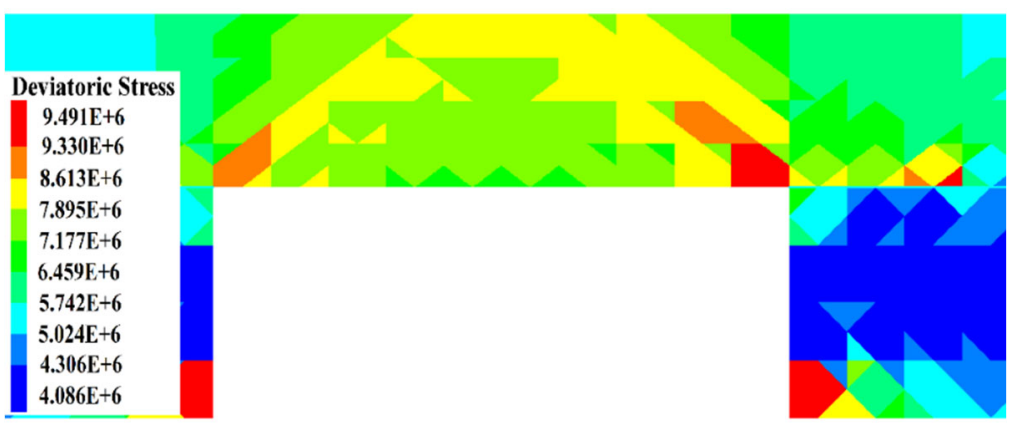

Fig. 7.11. Distribution of deviatoric stress after $7 \mathrm{~min}$. (unit: Pa) 


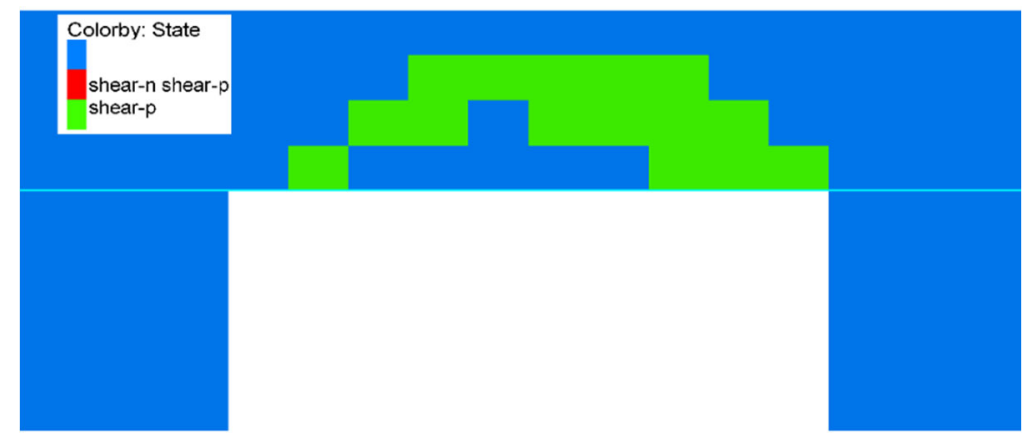

Fig. 7.12. Failure condition after 9 min.

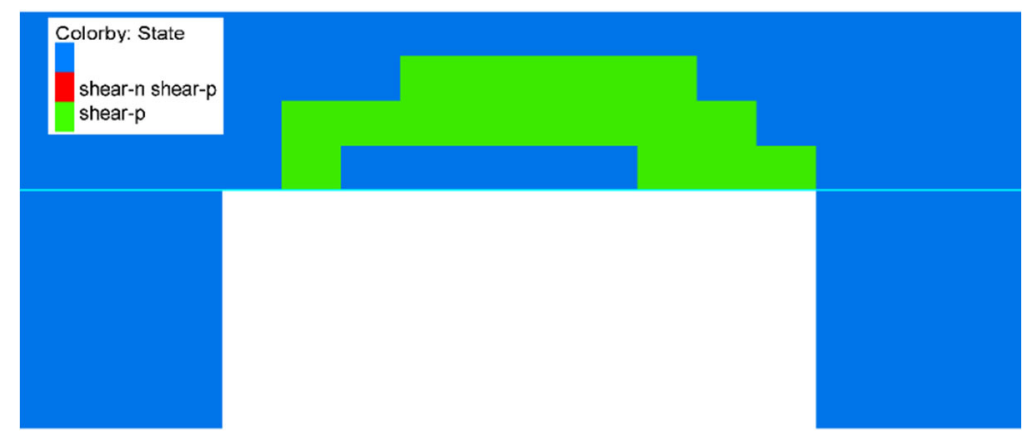

Fig. 7.13. Failure condition after $15 \mathrm{~min}$.

Figs. 7.14 and 7.15 plot the failure condition after 1 day and 1 month, respectively. Comparsion of the failure conditions after 15 minutues, 1 day, and 1 month demonstrates that there was failure propagation from 15 minutes to 1 day, while no failure propagation occurred from 1 day to 1 month. Failure only occurred within the immediate shale roof. From the distribution of major principal stress in Fig. 7.16, it is notable that the stress within the roof, especially the immediate roof, had relaxed significantly within 1 month. The low stress in the roof prevented further failure propagation with time. In addition, the stress relaxation within the mine roof always occurs in tandem with the viscous deformation. The accumulated viscous deformtion in turn deterioates the mine roof in the form of decreasing friction angle in the numerical simulation. Fig. 7.17 illustrates that significant reduction in friction angle occurred in the immediate roof; however, the reduction in cohesion was relatively much lower than the reduction in friction angle, which was resulted from the reduction mechanisms. The cohesion reduction is due to the accumulated shear plastic strain, which was further driven by stress. Along with the viscous deformation, the stress relaxed from the mine roof, furhter preventing plastic deformations. The friction angle, conversely, signficantly reduced as it was driven by the viscous deformation. Rock deterioration occurred along with the stress relaxation process within the mine roof. 


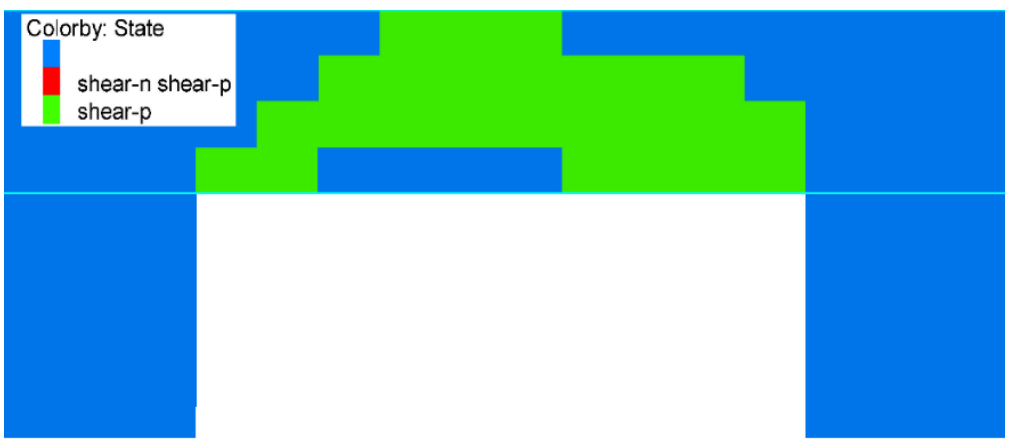

Fig. 7.14. Failure condition after 1 day

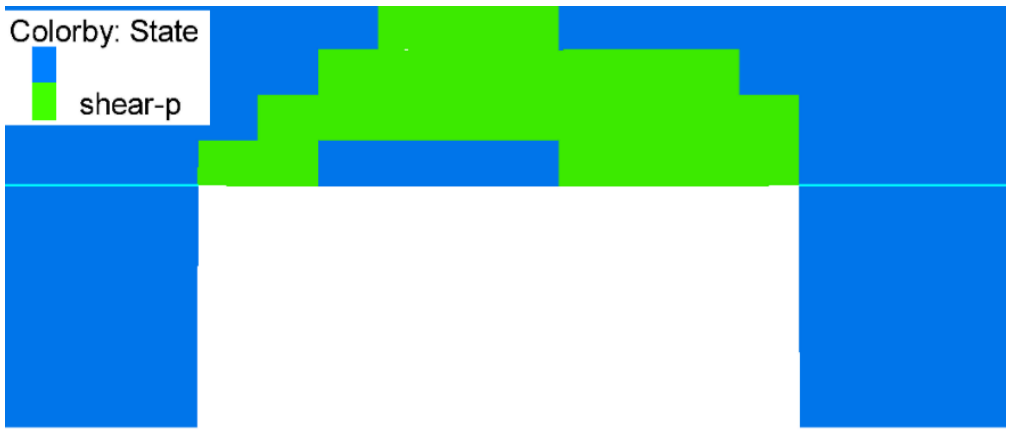

Fig. 7.15. Failure condition after 1 month

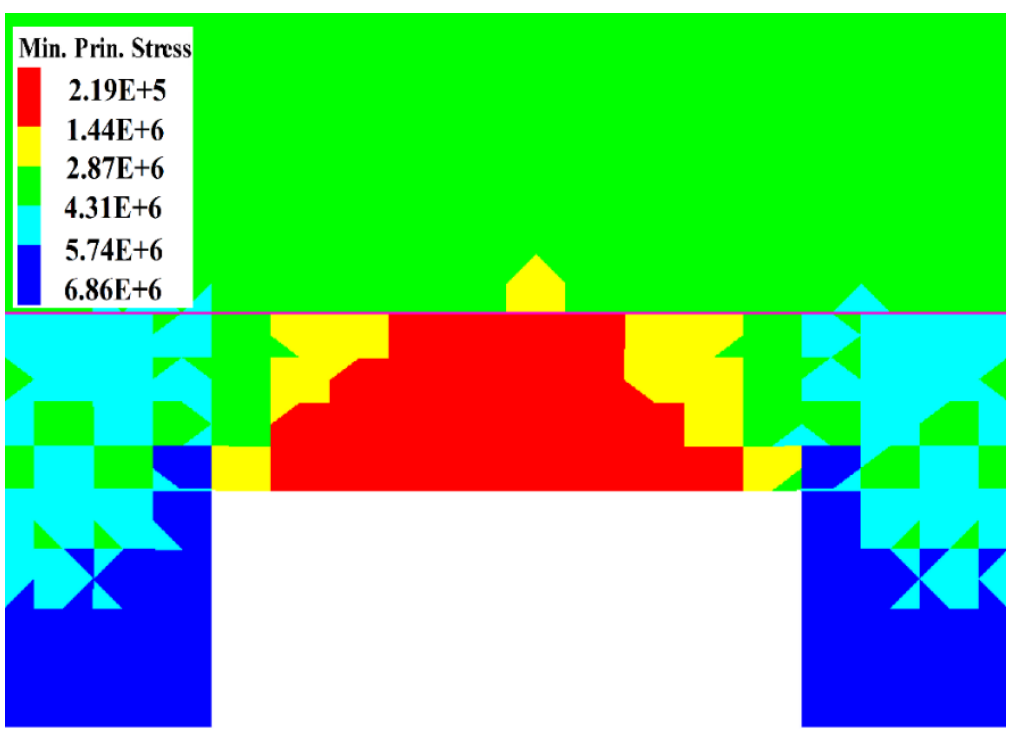

Fig. 7.16. Distribution of major principal stress after 1 month (unit: Pa) 


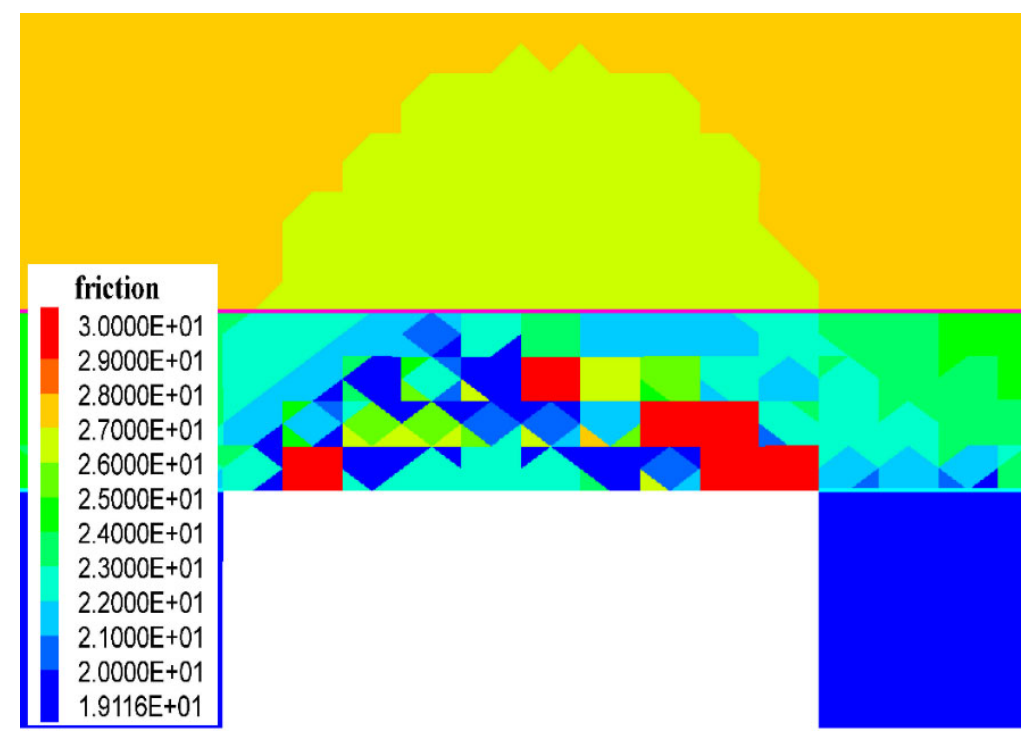

Fig. 7. 17. Distribution of friction angle after 1 month (unit: degree)

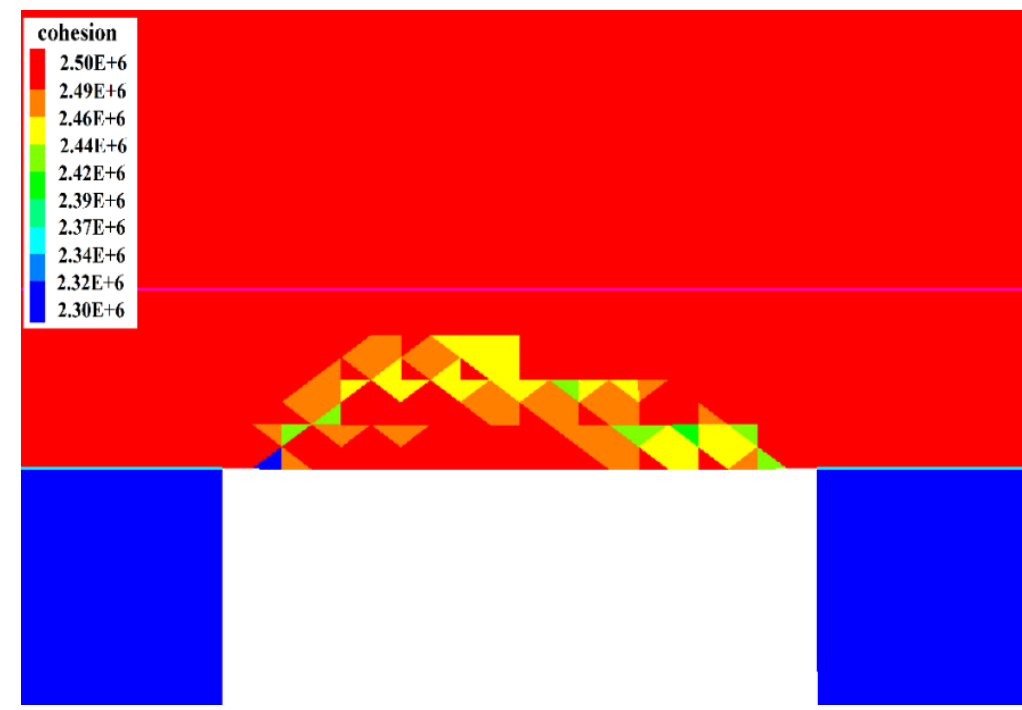

Fig. 7.18. Cohesion distribution after 1 month (unit: Pa)

This study monitored the time-dependent deformation within the mine roof during the creep simulation by placing a few monitoring points along a vertical line, $7.62 \mathrm{~m}(25 \mathrm{ft})$ inside of the entry and aligned with the center of the entry. Figs. 7.19 and 7.20 present the time-dependent displacement of the monitoring points, representing the mine roof convergence, after 1 day and 1 month, respectively. The recorded time-dependent displacements were normalized to the displacement of the roof at equilibrium, which is the displacement of the monitoring point on the roof line. Fig. 7.19 shows that the roof deformed with time and the deformations varied with location. On one hand, all the monitoring points demonstrated time-dependent deformation, indicating that the roof was deforming with time, regardless of the magnitude of the timedependent deformation. On the other hand, the time-dependent deformation varied with location. At equilibrium condition, representing the condition when time is zero in Fig. 7.19, the time-independent or instantaneous deformation after excavation gradually decreased with increasing depth into the roof. With the monitoring point located $2.4 \mathrm{~m}$ into the roof, the instantaneous deformation was less than $70 \%$ of the 
deformation on the roof line. In addition, the time-dependent deformation showed similar trends as a creep curve. The deformation rate decreased rapidly in the first stage and almost maintained constant in the second stage. However, at different locations, the displacement at the end of primary stage and the deformation rate in the secondary stage were different, decreasing with their locations deeper into the roof. The variation in the time-dependent deformation with the depth into the roof should be a result of the stress redistribution. In general, the confining stress releases from the mine roof, and the released stress gradually decreases with the increase in depth. The release of confining stress changes the stress distribution and increases the possibility of time-dependent deformation.

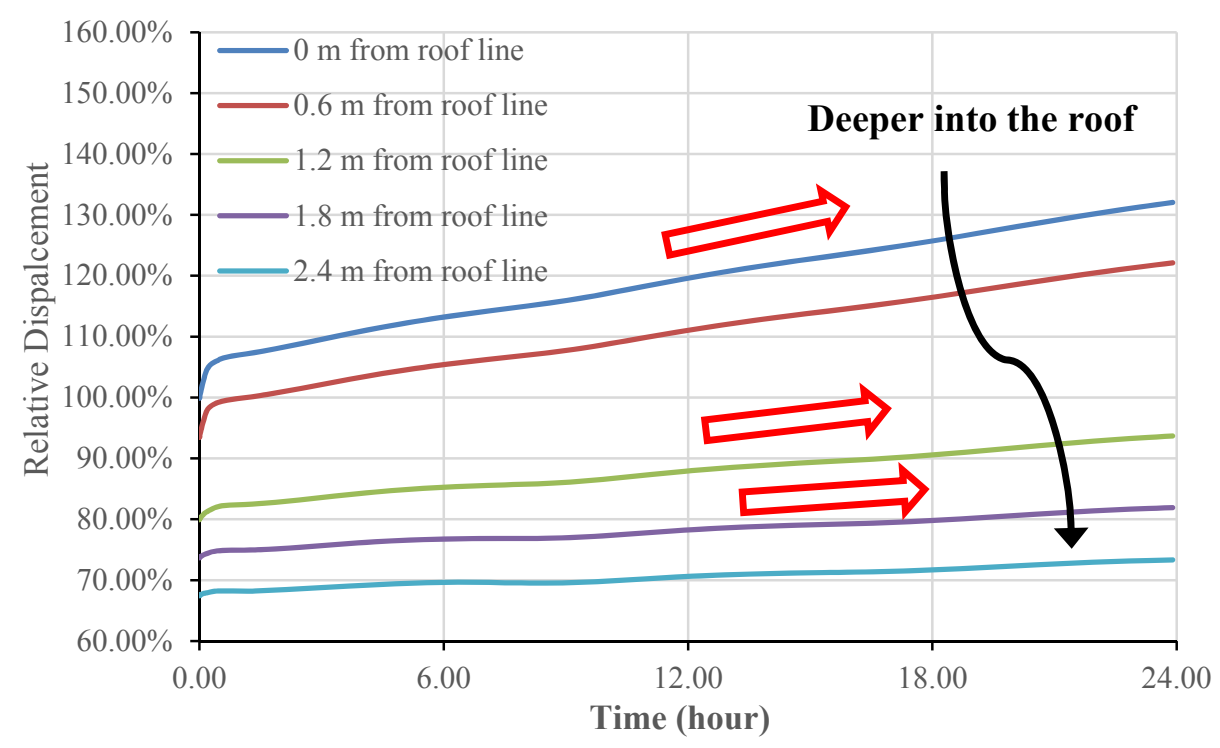

Fig. 7.19. Plot of time-dependent displacement of monitoring points after 1 day

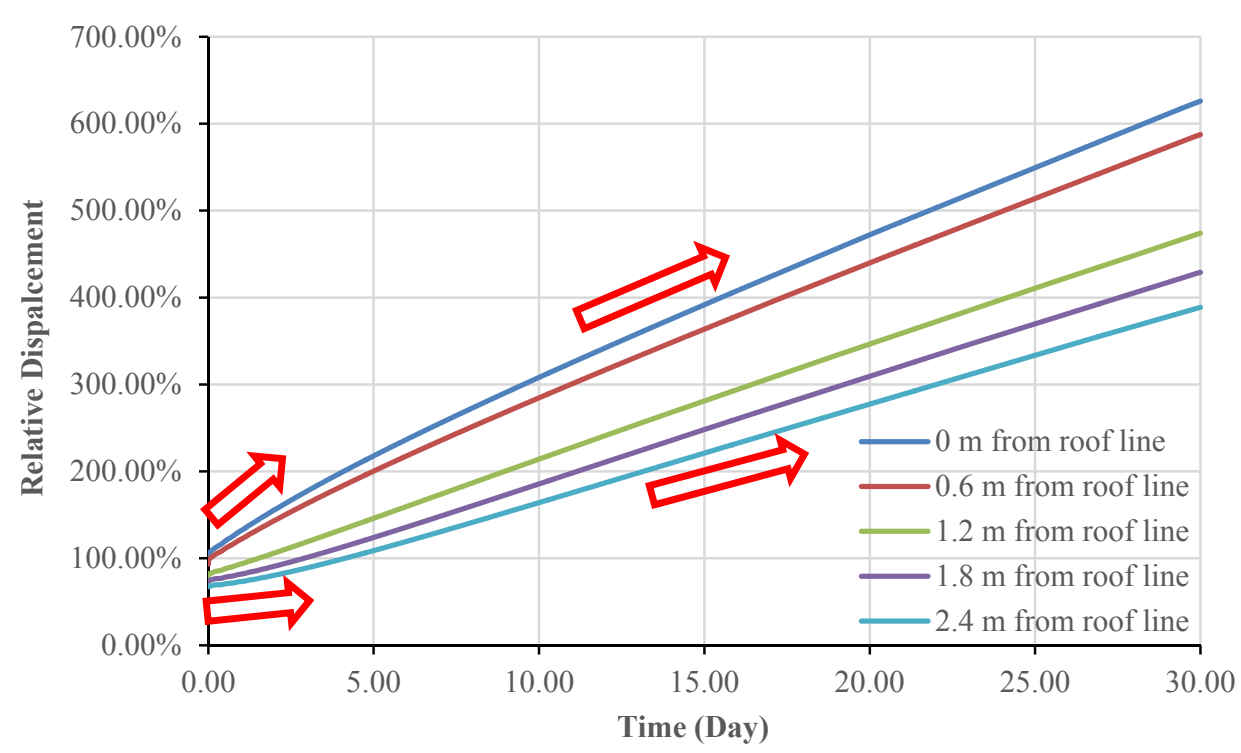

Fig. 7.20. Plot of time-dependent deformation of the monitoring points after 1 month

Furthermore, the comparison of Figs. 7.19 and 7.20 shows that the time-dependent deformation changes with time. Fig. 7.19 demonstrates the time-dependent deformation after 1 day of excavation, which 
represents the short-term response of the mine roof, while the displacement rate changed at different locations within one month. Fig. 7.20 illustrates that the deformation rate of the first two monitoring points, $0 \mathrm{~m}$ and $0.6 \mathrm{~m}$ from the mine roof, was large and gradually decreased with time in the secondary stage. However, the deformation rate of the other three monitoring points was small at the beginning of the secondary stage and gradually increased with time; this indicates that the deformation of the mine roof surface is slowing down while the deformation inside the roof is gradually accelerating. If using the deformation rate as an indicator of the stress relaxation, this demonstrates that the occurance of stress relaxation at the shallow locations of the mine roof gradually releases the confining stress of the deeper locations and accelerates the time-dependent deformation at the deeper locations. Therefore, the timedependent deformation inside the mine roof varies with time and the location (depth) from the roof line.

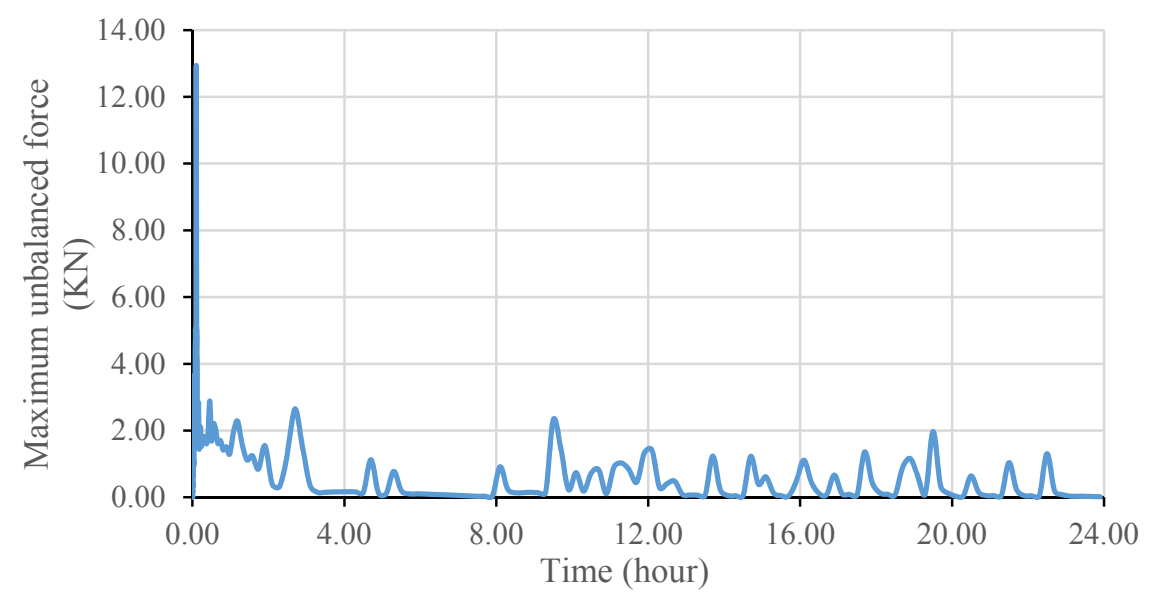

Fig. 7.21. Plot of maximum unbalance force during creep simulation of 1 day

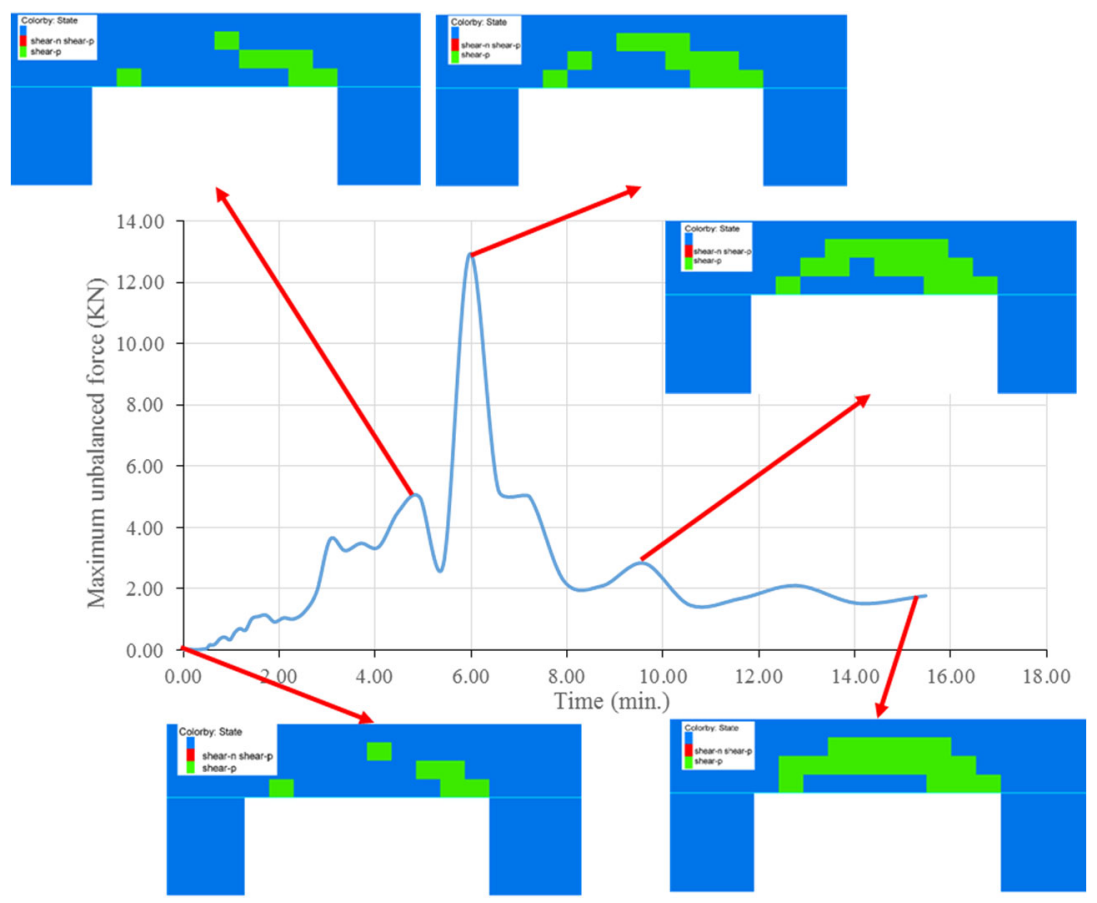

Fig. 7.22. Plot of maximum unbalance force during creep simulation of $15 \mathrm{~min}$. 
Finally, the maximum unbalanced forces was monitored during the creep simulation. Fig. 7.21 shows the maximum unbalanced force within 1 day of creep simulation. It is notable that the maximum unbalanced force was almost zero at the beginning of the creep simulation. The unbalanced forcre gradually increased with time and then decreased after one peak. Fig. 7.21 displays multiple peaks during one day's creep simulation. The figure shows the unbalanced force accumulating during the creep simulation and, at some point, the unbalanced force released. Fig. 7.22 shows the relation between unbalanced force and time in the first 15 minutes of creep simulation, and the failure conditions shown in Figs. 7.3, 7.7, 7.8, and 7.9 are maked at the approximate time. The time of the peak of the maximum unbalanced force corresponded to the time of failure propagation. Therefore, the unbalanced force accumulates during the visous deformation of the mine roof and releases once there is failure propagation.

\subsection{INFLUNECE OF STRESS RATIO ON THE TIME-DEPENDENT FAILURE OF MINE ROOF}

The purpose of this section is to investigate the influence of stress ratio on the time-dependent failure of mine roof. This study maintained the vertical stress and the minimum horizontal stress constant and changed the maximum horizontal stress to investigate the influence of stress ratio of maximum horizontal stress to minimum horizontal stress on the time-dependent response of mine roof. The following table lists the scenarios used in the simulations.

Table 7.1 In-situ stress scenarios used in the simulations

\begin{tabular}{|c|c|c|c|c|}
\hline $\begin{array}{c}\text { Stress } \\
\text { Scenario } \\
\text { No. }\end{array}$ & $\begin{array}{c}\text { Stress Direction- } \\
\text { Angle (degree) }\end{array}$ & $\begin{array}{c}\text { Horizontal } \\
\text { Stress-Maximum } \\
\text { (MPa) }\end{array}$ & $\begin{array}{c}\text { Horizontal } \\
\text { Stress-Minimum } \\
\text { (MPa) }\end{array}$ & $\begin{array}{c}\text { Vertical } \\
\text { Stress (MPa) }\end{array}$ \\
\hline $\mathbf{1}$ & 30 & 5.22 & 3.85 & 2.76 \\
\hline $\mathbf{2}$ & 30 & 6.55 & 3.85 & 2.76 \\
\hline $\mathbf{3}$ & 30 & 6.89 & 3.85 & 2.76 \\
\hline
\end{tabular}

Table 7.1 shows the three different stress ratios selected for the simulations. The simulation considers the first stress scenario to be the base case (Ray, 2009). The maximum and minimum horizontal stress were 5.22 $\mathrm{MPa}$ and $3.85 \mathrm{MPa}$, respectively. A stress ratio of 1.36 was obtained for this scenario. For the second and third scenarios, the simulations maintained the minimum horizontal stress and vertical stress constant, and the maximum horizontal stress increased from 5.22 $\mathrm{MPa}$ to $6.89 \mathrm{MPa}$. The horizontal stress ratio correspondingly increased from 1.36 to 1.79 .

The following figures plot the failure condition at equilibrium and after one day, and the distribution of maximum shear stress at equilibrium for stress scenario 1. There is a small failed area above the right upper corner of the entry within the roof. The distribution of the maximum shear stress shows that, starting from the two upper corners of the entry, a stress-arch forms in the roof and high stress concentration occurs at the two upper corners. Under this circumstance, failure only occurs above the right corner. It is notable that, except for the high stress concentration directly above the two upper corners, the maximum shear stress within the stress arch is relatively low with a range between 2.87 and $3.59 \mathrm{MPa}$. After the creep simulation of one day, there was no propagation of the failure initiating from the right upper corner, and there was failure initiating from the left upper corner due to the high stress concentration. 


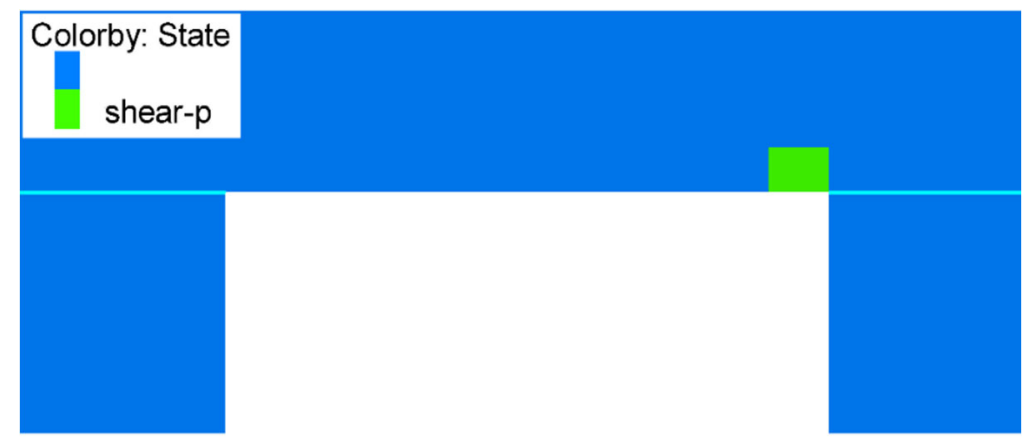

Fig. 7.23. Failure condition at equilibrium for stress scenario 1

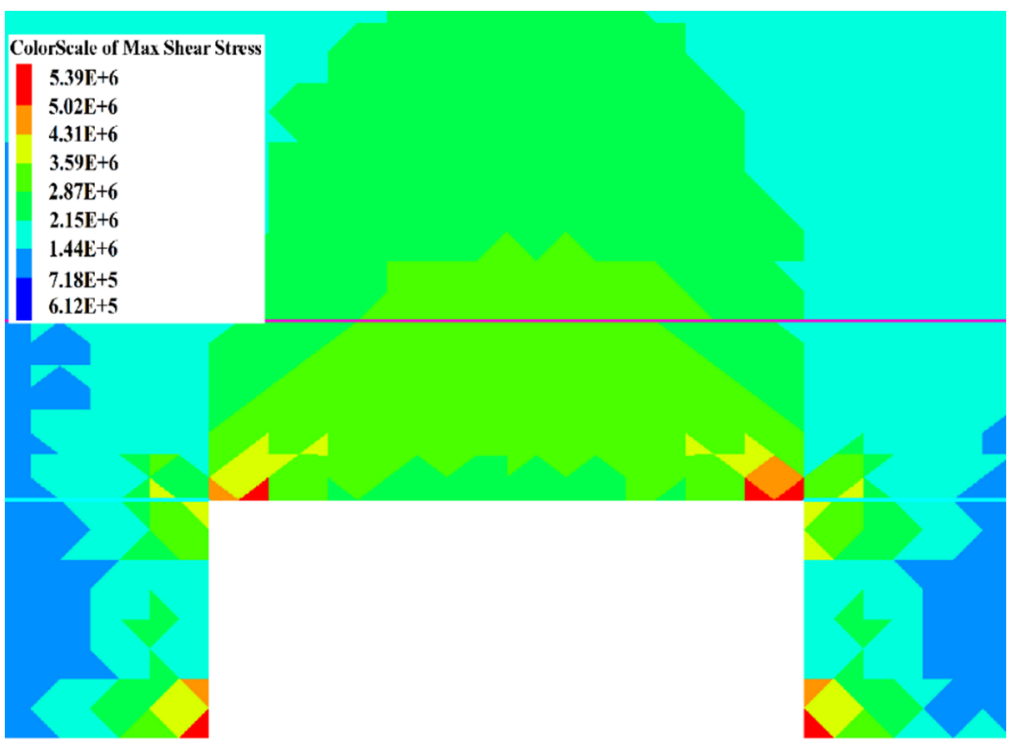

Fig. 7.24. Distribution of maximum shear stress at equilibrium for stress scenario 1 (unit: Pa)

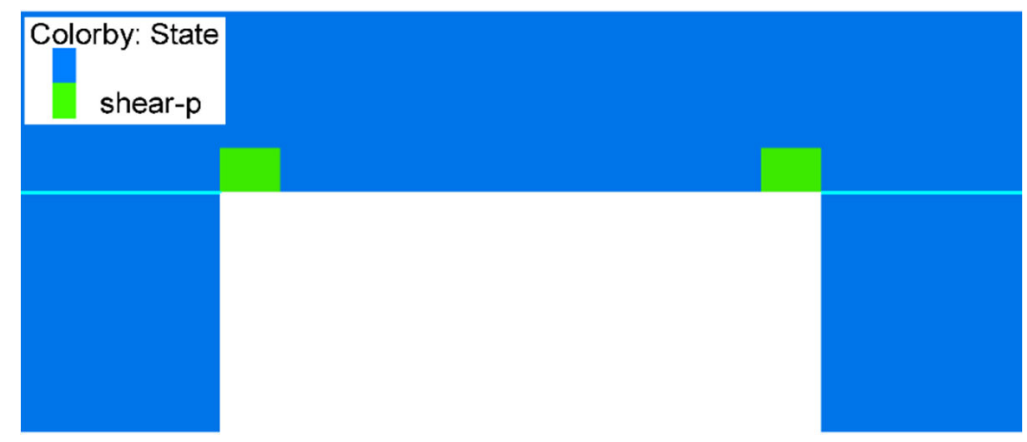

Fig. 7.25. Failure condition after 1 day for stress scenario 1

In addition, Figs. 7.3 and 7.14 show the failure condition at equilibrium and after one day for stress scenario 2, respectively. The comparison of the failure condition between stress scenarios 1 and 2 shows that the increase of maximum horizontal stress increases the failure area within the mine roof. The increase in maximum horizontal stress of $1.33 \mathrm{MPa}$ initiated the failure above the left upper corner and promoted the failure propagation deep into the roof initiating from the right upper corner. Also, the failures initiating from the two upper corners gradually propagated with time and coalesced to form a failure arch within the mine roof. From stress scenario 1 to stress scenario 2, the increase of $1.33 \mathrm{MPa}$ in maximum horizontal stress does not significantly increase the failure at equilibrium; however, there is a pronounced increase in 
the failure in the creep simulation. Examining the distribution of maximum shear stress for stress scenario 2 led to a similar distribution as in Fig. 7.24 with a stress arch existing in the immediate roof. However, the stress range for the stress arch is between 3.83 and 4.59 MPa for stress scenario 2. Although there was no pronounced change in the stress distribution, the stress magnitude significantly increased, accelerating the viscous deformation and thus increasing the degradation of the rock. The increase in the stress magnitude also indicates that the stress state is close to failure. Along with the rock degradation, the rock may fail with time. Therefore, the increase in the stress magnitude results in the difference in the instantaneous and timedependent failure between stress scenarios 1 and 2.

Furthermore, Figs. 7.26, 7.27, and 7.28 plot the failure condition at equilibrium and after one day, and the distribution of maximum shear stress at equilibrium for stress scenario 3, respectively. A failure arch already formed within the mine roof at equilibrium and high maximum shear stress surrounded the failure arch. The failure propagated horizontally in the following creep simulation.

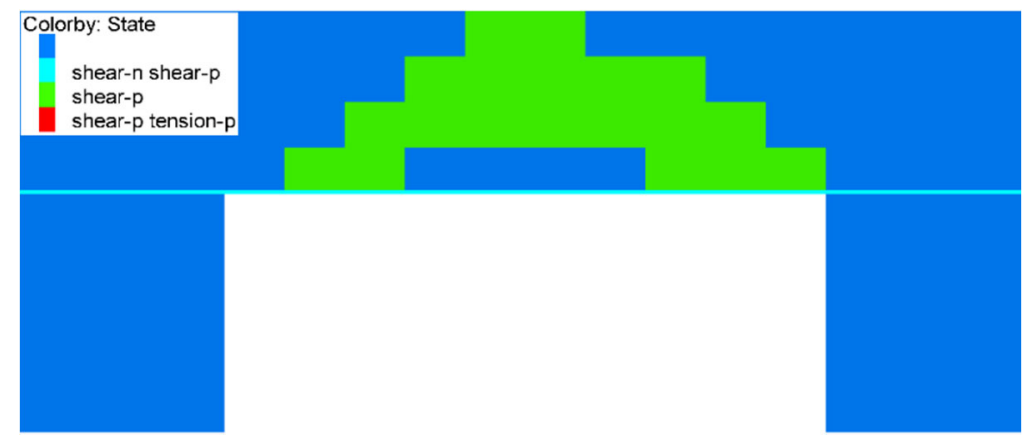

Fig. 7.26. Failure condition at equilibrium for stress scenario 3

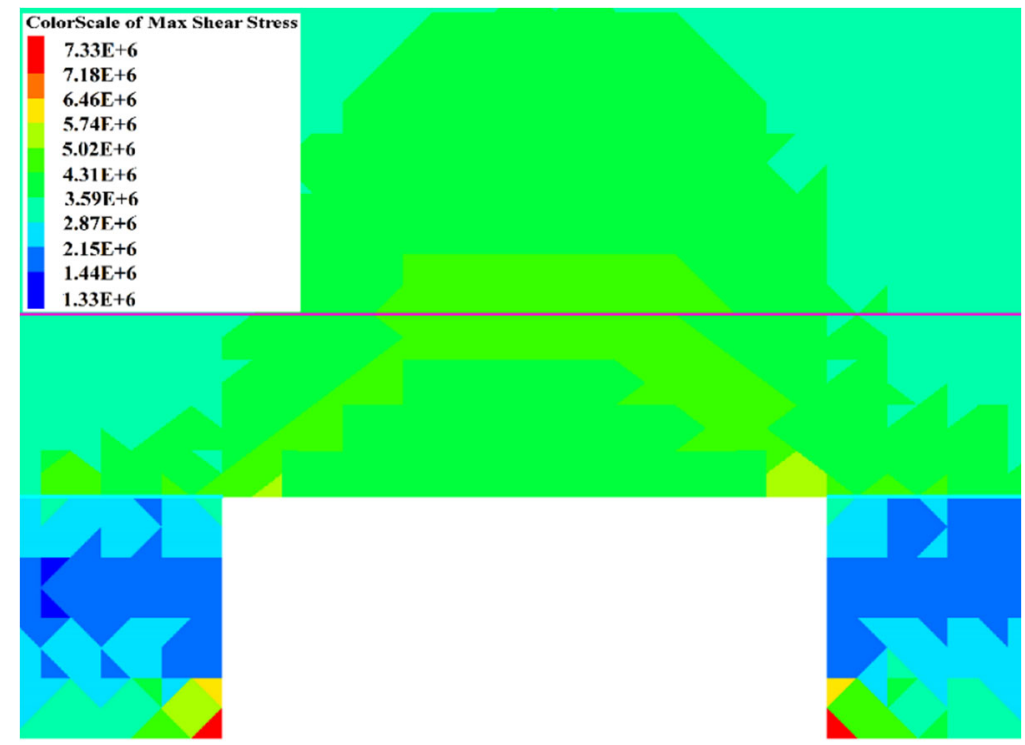

Fig. 7.27. Distribution of maximum shear stress at equilibrium for stress scenario 3 (unit: Pa)

The influence of stress ratio on the instantaneous and time-dependent failure of mine roof changed from stress scenario 2 to situation 3. The slight increase in the maximum horizontal stress significantly changed the failure condition at equilibrium. For stress scenario 3, the failures initiating from the two corners already 
coalesced at equilibrium, which further affected the stress distribution. Once the zone fails, it cannot hold extra load, which then transfers to adjacent intact zones and lead to the increase of stress in the adjacent areas. Fig. 7.27 displays the high maximum shear stress surrounding the failed area. After the coalescence of the failures, the failure propagates horizontally with time. This observation demonstrates that the influence of stress ratio on the time-dependent failure of mine roof also depends on the failure condition.

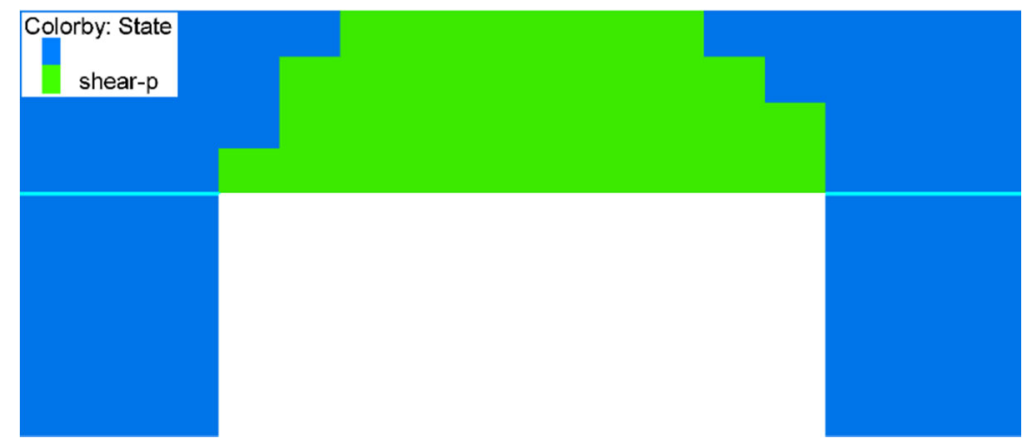

Fig. 7.28. Failure condition after 1 day for stress scenario 3

\subsection{INFLUENCE OF STEP-WISE EXCAVATION ON THE TIME-DEPENDENT FAILURE OF MINE ROOF}

In the previous simulation of the time-dependent failure of mine roof, the entry was entirely mined in one step and then the model ran to equilibrium. However, the development of mine entry is a timedependent process, as it cannot mine the whole entry instantaneously. In addition, the continuous mining process always involves rock failure and plastic deformation, which is stress-path dependent. The realistic simulation of the mining process can obtain better failure profile of the mine roof.

But how is it possible to incorporate time into the mining process in numerical simulation? There are timesteps in the numerical simulation of time-dependent and time-independent behaviors; however, the meaning of the timestep for these two situations is different. For time-independent simulation, the timestep is a method to bring the system to reach mechanical equilibrium condition; as such, it does not represent real time. The length of the time-step is determined to ensure the convergence of the finite differential equations. The main 3DEC software uses this timestep. However, the timestep in time-dependent simulations represents real time. If the length of the time-step is 1 second, the model should process 10 seconds when the model runs for 10 steps. The constitutive model incorporates this timestep into timedependent simulations. When setting timestep to zero, the new stress values calculated by the constitutive model at each timestep represent the elastic or elastoplastic response. If this timestep is not zero, the calculated new stress values include the viscous response. The current procedure for time-dependent simulation includes the following steps: (1) assign the timestep length as zero (by default) and run the model to equilibrium condition to obtain the elastic or elastoplastic response of the model and (2) assign the creep time-step length with a nonzero value and run the model to a determined time to obtain the time-dependent response of the model. Therefore, two kinds of timestep are used in the creep simulation of underground excavations; one is used to bring the model to equilibrium condition and get the instantaneous response of the excavation and the other one is to introduce real time into the simulation and show the time-dependent response after excavation. 
When considering the entry development to be a continuous process, how can time be incorporated into the numerical simulation of the continuous process? The methodology in this section intends to simulate the step-wise excavation. Each step excavates one section of entry. The model runs to equilibrium and the time-dependent simulation runs for the corresponding time of excavation. By reducing the length of section to infinitely small, the mining process becomes continuous; however, this is impossible in numerical simulation. Instead, small lengths of section can approximate the continuous process. The following simulations used one length of $0.3 \mathrm{~m}(1 \mathrm{ft})$.

Another factor potentially affecting the time-dependent failure of mine roof is the mining speed. Lower mining speed always involves more time to excavate the entry of the same length. It means that more timedependent deformation and even failure may occur with lower mining speed. Therefore, the mining speed affects the time-dependent response of the mine roof. After mining $0.3 \mathrm{~m}$ of entry and running to equilibrium, the model ran for a period of time under time-dependent condition to represent the mining speed. The following simulations used a mining speed of $2.4 \mathrm{~m}(8 \mathrm{ft})$ per hour.

As the simulation set the displacement-monitoring points at the location of $7.62 \mathrm{~m}(25 \mathrm{ft})$ along the entry from the front surface of the model, the analysis of the influence of step-wise excavation on the timedependent response of mine roof mostly focused on the cross-section at this location. This study analyzed the failure condition, displacement distribution and distribution of cohesion and friction angle at this location when the face was $0.30 \mathrm{~m}(1 \mathrm{ft}), 1.52 \mathrm{~m}(5 \mathrm{ft})$, and $3.05 \mathrm{~m}(10 \mathrm{ft})$ past the monitoring location.

(1) The $26^{\text {th }}$ mining step

Fig. 7.29 plots the failure condition at the cross-section of the monitoring location after the $26^{\text {th }}$ mining step. When the mining face passed the monitoring location for $0.30 \mathrm{~m}$, the failure initiated from the right corner of the entry and the failure only occurred in a small area. Fig. 7.30 plots the distribution of the maximum shear stress along the right edge of the entry. This figure shows that the excavation had disturbed the stress distribution within the mine roof; there was an increase in the maximum shear stress within the roof, and high maximum shear stress existed in the immediate roof. In addition, the location of the mining face affects the distribution of the high maximum shear stress in the immediate roof. The coal at the mining face supports the roof, and the immediate roof close to the mining face cannot move downward freely. The constraint from the mining face gradually decreases with the increasing distance from the mining face. Fig. 7.30 shows that high stress existed close to the roof line in the immediate roof that is a few meters behind the mining face. However, the high stress gradually shifted to a deeper area into the mine roof when the location was far from the mining face. Based on the observation in the stress distribution, it is likely that with the pass of the mining face, the stress within the immediate roof would first increase and then decrease. The stress gradually shifted to a deeper roof area along with the failure of roof rocks close to the roof line. 


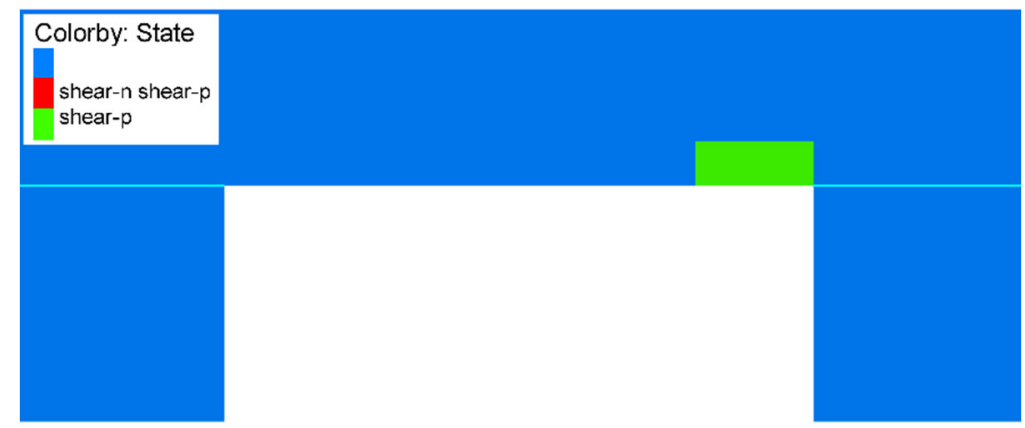

Fig. 7.29. Failure condition at the monitoring location after the 26 th $(26 \mathrm{ft})$ mining step

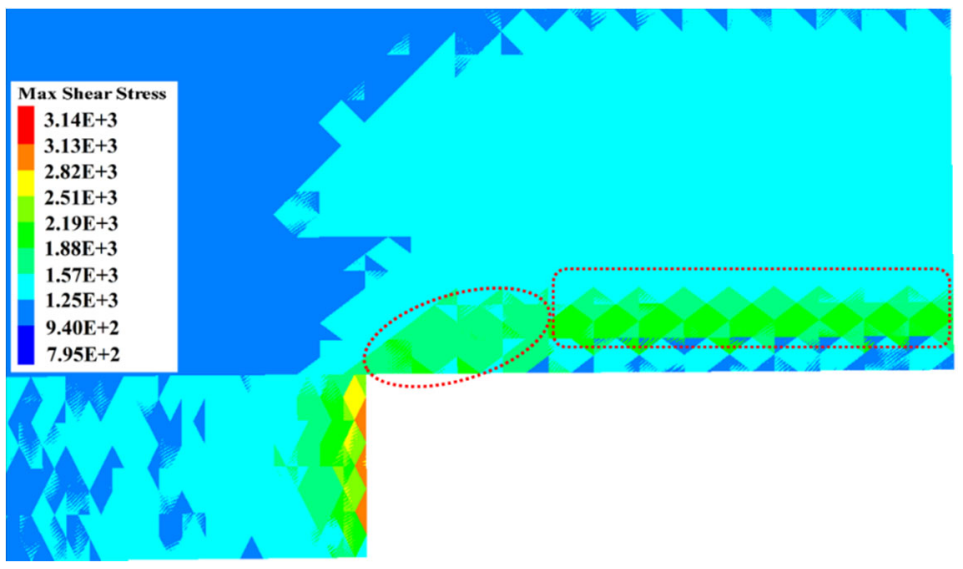

Fig. 7.30. Distribution of maximum shear stress along the right edge of the entry after the 26th (26 ft) mining step (unit: $\mathrm{Pa}$ )

In addition, Fig. 7.31 plots the distribution of friction angle at the monitoring location after the $26^{\text {th }}$ mining step. The property of the immediate roof above the opening deteriorates in the form of decreasing friction angle. Although this affected the immediate roof above the opening, the deterioration concentrated within the bottom layer and above the right upper corner of the entry. The distribution of friction angle shows that the deviatoric stress should be high within these areas. The high stress induces rock deterioration within these areas, leading failure to propagate towards them.

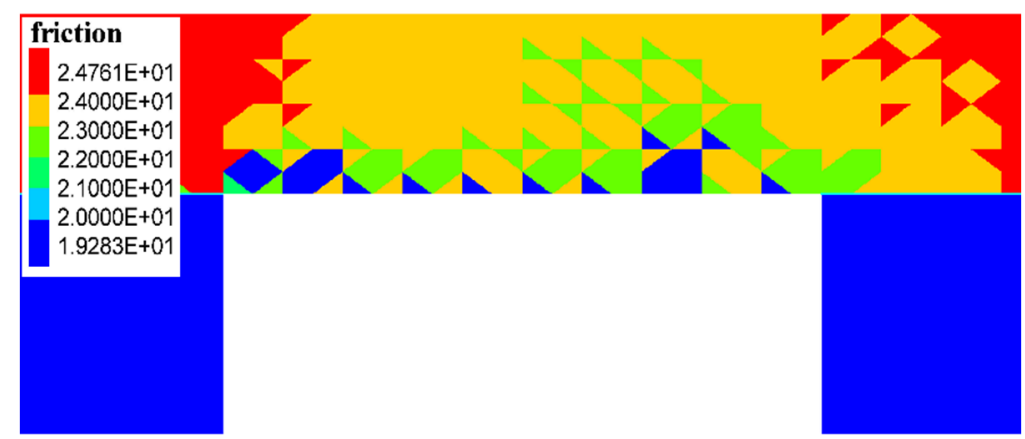

Fig. 7.31. Distribution of friction angle at the monitoring location after the 26th (26 ft) mining step (unit: degree)

Furthermore, Fig. 7.32 plots the distribution of displacement at the monitoring location after the $26^{\text {th }}$ mining step. It is notable that the maximum displacement within the roof occurred at the center of the roof. Due to the unloading effect of the excavation, the center of the immediate roof had the least confinement and achieved the maximum displacement within the roof. 


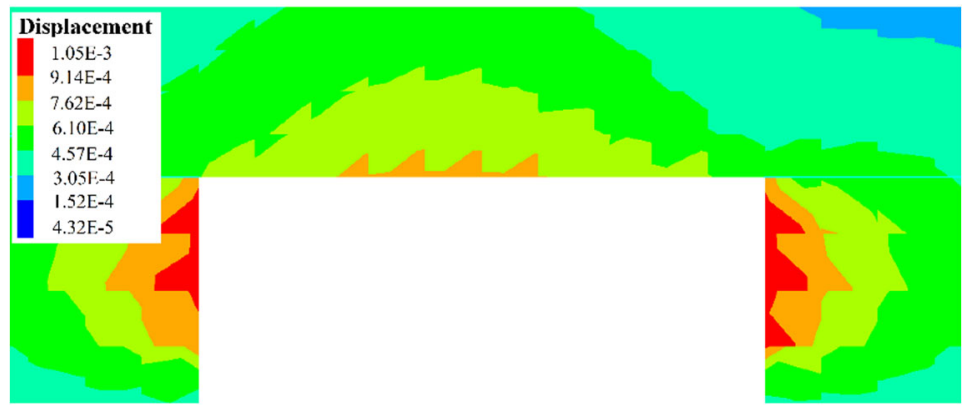

Fig. 7.32. Distribution of displacement at the monitoring location after the 26 th $(26 \mathrm{ft})$ mining step (unit: degree)

(2) The $30^{\text {th }}$ mining step

According to the analysis after the $26^{\text {th }}$ mining step, the stress at the monitoring locations would increase when the face passed. Due to the constraint from the mining face, there is no extensive failure occurrence. However, the increase in stress would induce the deterioration of roof rocks. The failure condition at the monitoring location after the $30^{\text {th }}$ mining step in Fig. 7.33 shows that the failure initiating from the right upper corner of the entry gradually propagated in two directions, corresponding to the strength deterioration observed in Fig. 7.31. Due to the unloading by mining and the release of constraint from the mining face, the first layer of the immediate roof failed; at the same time, due to the directional horizontal stress, the stress concentration above the right upper corner induced the failure propagation into deeper roof area. Along with the failure propagation, shear plastic strain accumulated within the failed area and further decreased the rock strength in the form of reducing cohesion. The accumulation of shear plastic strain is dependent on the stress distribution at that time. Fig. 7.34 shows that although the first layer of the roof had already failed, cohesion reduction mostly occurred at the areas above the entry corners, where stress concentration occurred.

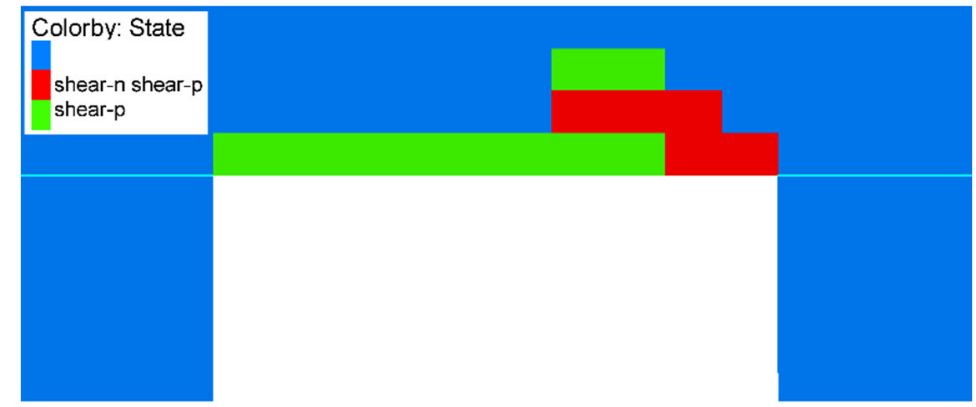

Fig. 7.33. Failure condition at the monitoring location after the 30th (30 ft) mining step

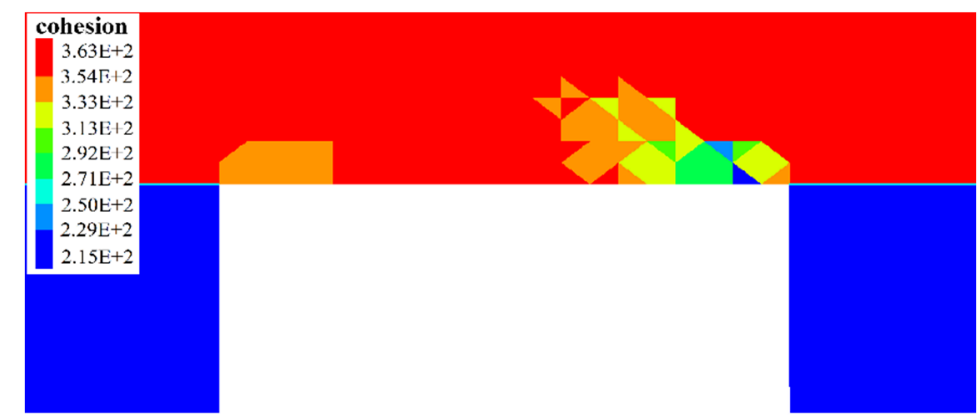

Fig. 7.34. Cohesion distribution at the monitoring location after the 30th (30 ft) mining step (unit: Pa) 
In addition, Fig. 7.35 plots the distribution of friction angle at the monitoring location after the $30^{\text {th }}$ mining step. The distribution shows that the rock deterioration mostly occurred within the roof above the opening with additional deterioration occurring at the areas surrounding the failed zones. It is also important to note that the deterioration occurring above the left upper corner began to extend upwards, indicating the potentially upward failure propagation from the left upper corner.

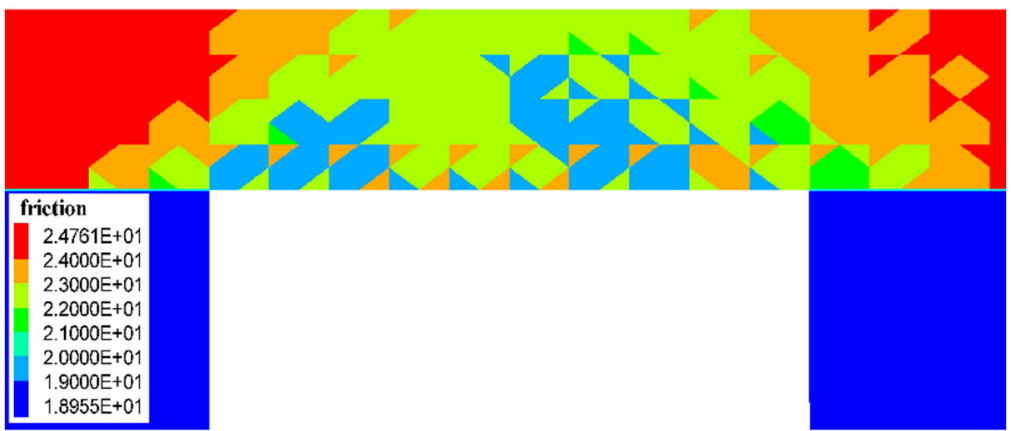

Fig. 7.35. Distribution of friction angle at the monitoring location after the 30th (30 ft) mining step (unit: degree)

Furthermore, there is a change in the distribution of displacement along with the advancing of the mining face. The displacement distribution in Fig. 7.36 shows a shift in the location with maximum displacement within roof. Located at the center of the roof after $26^{\text {th }}$ mining step, it shifted to the right side of the entry. This feature is visible by comparing Figs. 7.33 and 7.36, showing that the shift of the displacement is the result of the failure initiating from the right upper corner. The failure of this area led to the decrease of viscous parameters, and more viscous deformation could occur with same stress condition. The deformation in this area would then affect the displacement in the surrounding areas. Therefore, the failure occurring at one side of the roof changed the displacement distribution of the whole immediate roof.

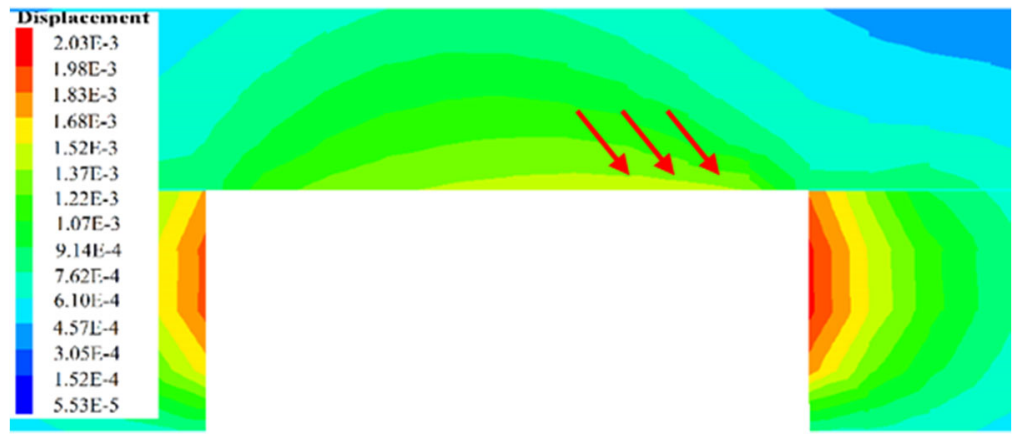

Fig. 7.36. Displacement distribution at the monitoring location after the 30th (30 ft) mining step (unit: $\mathrm{m}$ )

(3) The $35^{\text {th }}$ mining step

When the mining face advanced further, this study analyzed the condition of the immediate roof after the $35^{\text {th }}$ mining step. The failure condition in Fig. 7.37 shows that the failure continued propagating along with the advancing face and accumulated creep time. The failure initiating from the right upper corner propagated upwards and horizontally, while the failure initiating from the left upper corner began to propagate upwards. At the same time, the accumulated shear plastic strain continued reducing the strength of failed zones, as shown in Fig. 7.38. 


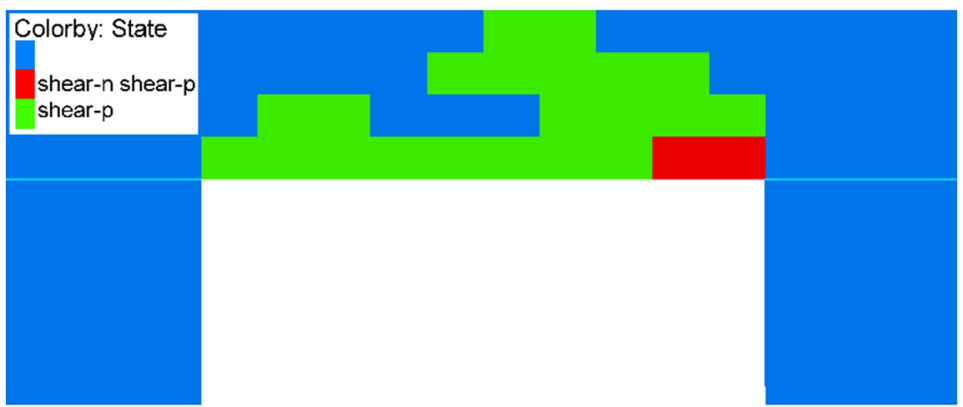

Fig. 7.37. Failure condition at the monitoring location after the 35 th ( $35 \mathrm{ft})$ mining step

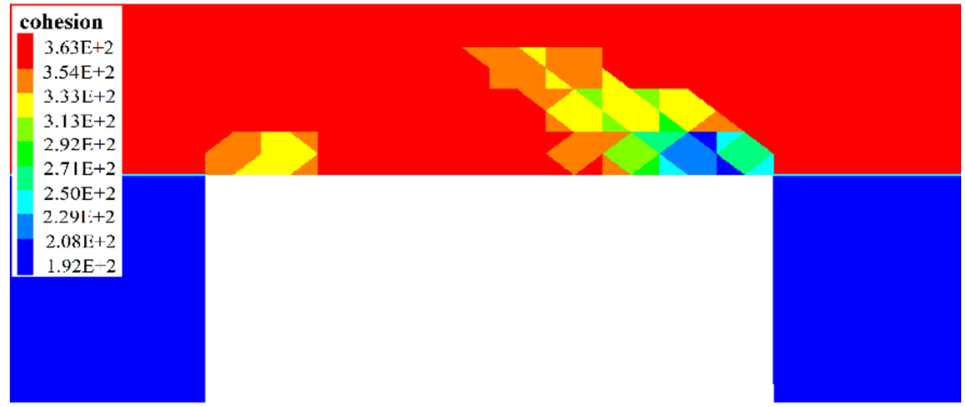

Fig. 7.38. Cohesion distribution at the monitoring location after the 35th (35 ft) mining step (unit: Pa)

In addition, the distribution of friction angle and displacement at the monitoring location after the $35^{\text {th }}$ mining step are plotted in Figs. 7.39 and 7.40, respectively. From the distribution of friction angle, it can be found that the strength deterioration concentrated to the area surrounding the failed zones. As the upper starata is strong, no failure occurred in the upper strata and the failure was expected to proparate horizontally. From the distribution of displacement, we can see that, after shiftting the location of maximum displacement to the right side, the displacement continued developing with time.

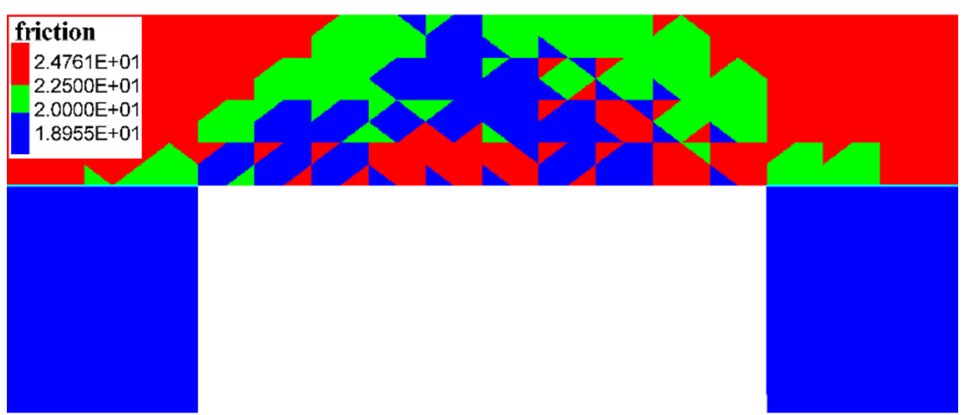

Fig. 7.39. Distribution of friction angle at the monitoring location after the 35 th ( $35 \mathrm{ft}$ ) mining step (unit: degree)

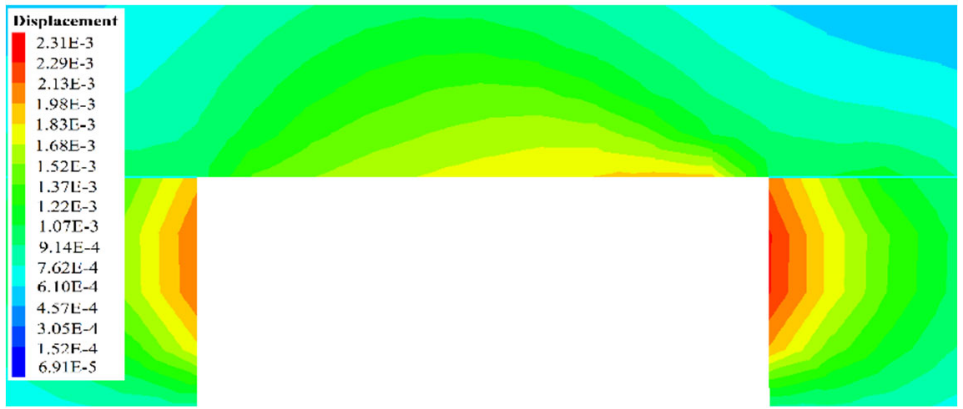

Fig. 7.40. Displacement distribution at the monitoring location after the 35th (35 ft) mining step (unit: $\mathrm{m}$ ) 
(4) The final condition after the $50^{\text {th }}$ mining step

As the response of the roof to the directional horizontal stress is anti-symmetric, this study excavated only half of the entry in a step-wise manner, meaning that the step-wise simulation mined 50 steps from the beginning. From the failure condition in Fig. 7.41, it is visible that the failure constrained in the immediate roof due to the above strong strata. Rather than propagating upwards, the failure propagated horizontally in the immediate roof after reaching the top of the immediate roof in Fig. 7.37. Furthermore, the roof continued deforming in the asymmetric manner caused by the failure within the mine roof, as shown in Fig. 7.42.

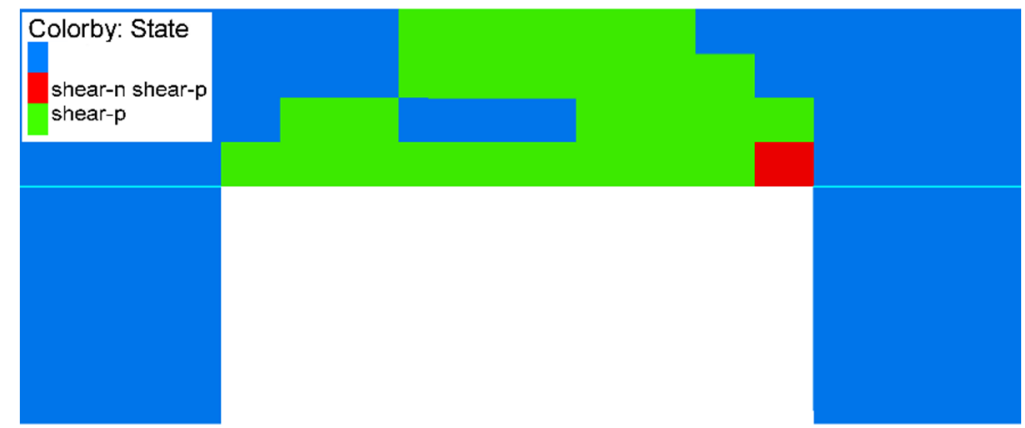

Fig. 7.41. Failure condition at the monitoring location after the 50th (50 ft) mining step

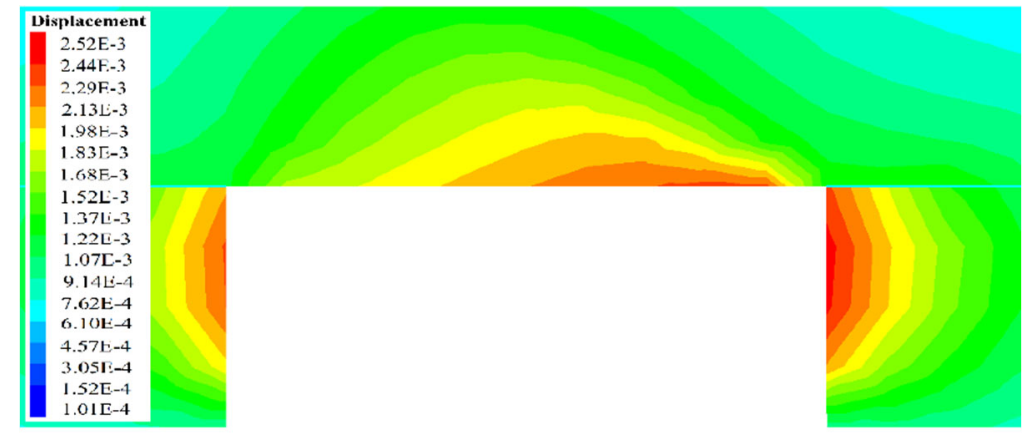

Fig. 7.42. Displacement distribution at the monitoring location after the 50th (50 ft) mining step (unit: $\mathrm{m}$ )

In addition, a few monitoring points with a $0.61 \mathrm{~m}(2 \mathrm{ft})$ interval were set along the center of the entry at the monitoring location. The vertical displacement of these monitoring points inside the mine roof was monitored during the step-wise excavation of the entry. From the relation between vertical displacement and time in Fig. 7.43, it is notable that the total vertical displacement and the deformation rate gradually increased when the mining face moved close and gradually decreased when the mining face passed far away. Most of the displacement occurred in the following few mining steps after the mining face passed, as marked by the dotted rectangle in Fig. 7.43. The instantaneous displacement and the time-dependent displacement between each mining steps during this period were much larger than others. Specifically, the roof started to deform a few mining steps before the mining face passed. The instantaneous deformation significantly increased in the few steps after the mining face passed, and the deformation at each step gradually decreased with increasing distance between the mining face and the monitoring location. It is important to note that the monitoring points located on the roof line was removed because of the excavation in the $25^{\text {th }}$ mining step and, as a result, the plot for that monitoring point became a horizontal line after $25^{\text {th }}$ mining step. 


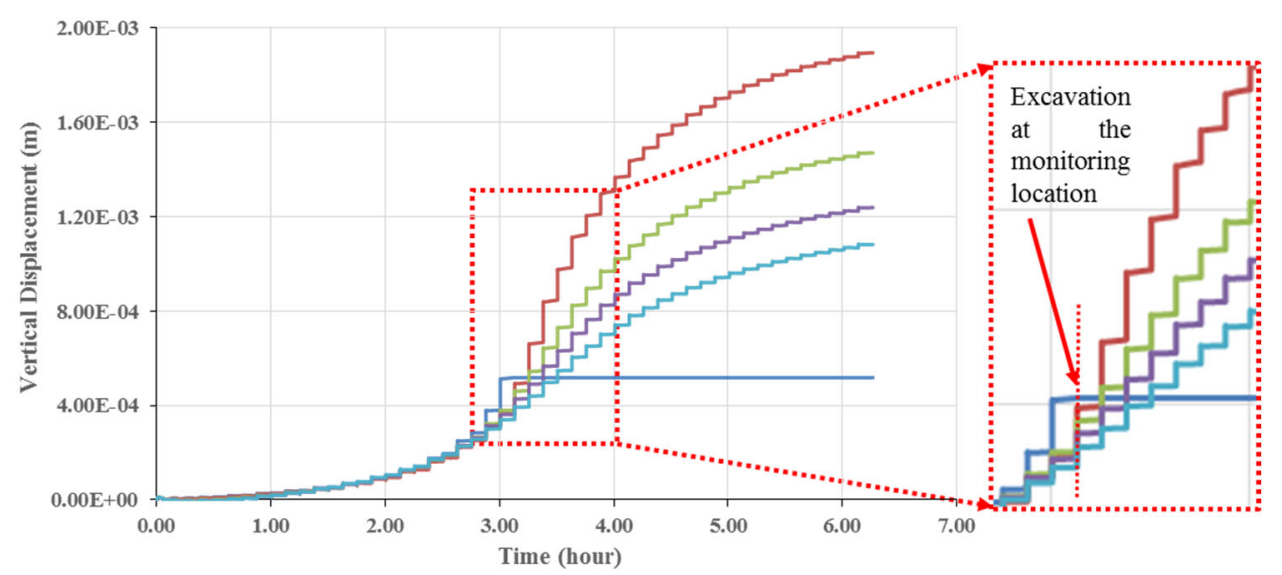

Fig. 7.43. The relation between displacement and time for the various monitoring points within the mine roof

In addition, Fig. 7.43 demonstrates that the instantaneous deformation resulted in most of the total displacement. The short time accumulated during the step-wise excavation limits the contribution of viscous deformation to the total displacement. This study also analyzed the displacement during the creep simulation between mining steps. Fig. 7.44 plots the vertical displacement of the monitoring point $0.61 \mathrm{~m}$ ( $2 \mathrm{ft}$ ) into the roof during the creep simulation after the $24^{\text {th }}, 25^{\text {th }}, 26^{\text {th }}, 30^{\text {th }}, 35^{\text {th }}$, and $50^{\text {th }}$ mining step. It is observed that from $24^{\text {th }}$ to $30^{\text {th }}$ mining step (the dotted lines), the deformation rate and the final deformation gradually increased; however, they gradually decreased from the $30^{\text {th }}$ to $50^{\text {th }}$ mining step. Therefore, the instantaneous displacement and time-dependent displacement were relatively large in the few mining steps after the mining face passed.

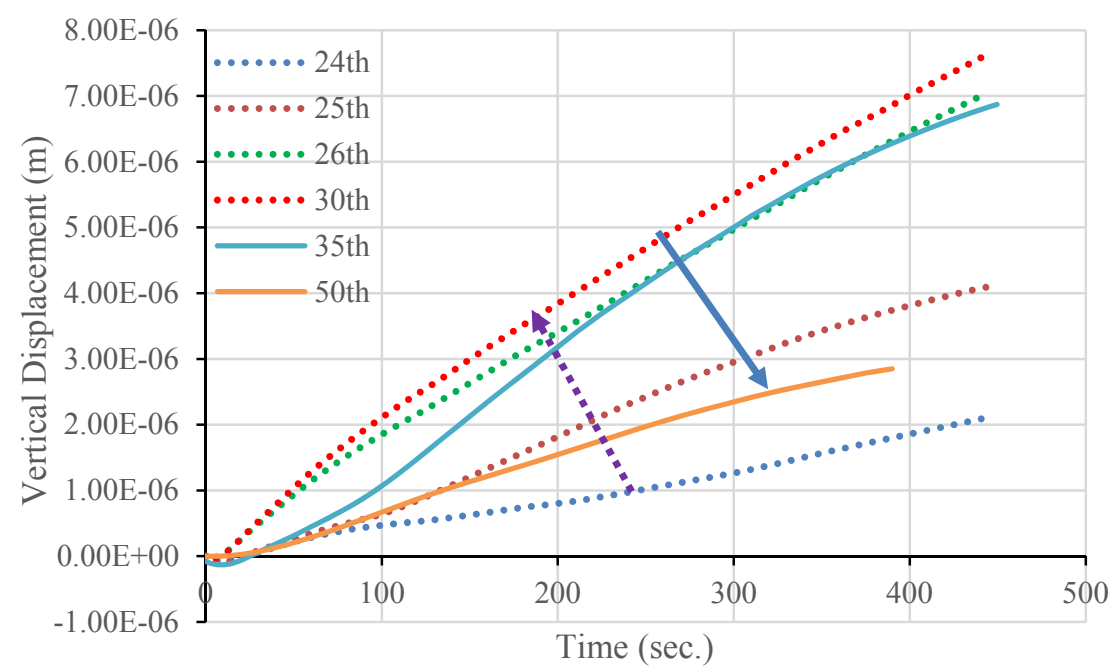

Fig. 7.44. The time-dependent vertical displacement of one monitoring point after six mining steps

\subsection{SUMMARY}

The formation of roof falls is a process involves time-independent behavior (stress redistribution due to excavation) and time-dependent behaviors. The creep simulation in 3DEC can simulate both behaviors and provides an approach to investigate the time-dependent roof failure. The time-dependent roof behavior in 
numerical simulations are mainly determined by the rock behavior and other "external" factors, like in-situ stress, mine layout and cutting sequence. Rocks show strain-softening behavior, time-dependent behaviors (including creep and relaxation), variation in the time-dependent property and strength deterioration with accumulated viscous deformation. Chapter 6 proposed and programmed a constitutive model that can represent all of these behaviors in 3DEC as a user-defined model. This chapter then used the model to simulate the time-dependent response of massive mine roof without bedding planes.

First, the new model simulated the time-dependent response of the mine roof with directional horizontal stress to demonstrate the time-dependent failure process. After excavation, failure initiates from the entry upper corners within the mine roof due to high stress concentration, and an arch-shaped band with high deviatoric stress exists within the immediate roof. Along with the accumulated viscous deformation, strength degradation occurs within the band; in turn, failure potentially propagates along the arch-shaped band. Once the elements fail, shear plastic strain gradually accumulates and further decreases the strength of failed rock. The failure propagates horizontally after the failures initiating from the entry upper corners coalesce along with the arch-shaped band. At the same time, stress gradually relaxes from the immediate roof, potentially preventing the further propagation of the time-dependent failure and accumulation of shear plastic strain. The monitored deformation shows that, along with the time-dependent stress distribution, the time-dependent deformation rate inside the mine roof varies with location (depth) from the roof line and time. The monitored maximum unbalanced force demonstrates that the unbalanced force accumulates during the viscous deformation and releases once there is failure propagation.

In addition, other "external" factors affecting the stress distribution around the mine entry affects the time-dependent roof stability. Directional horizontal stress, one of the key factors affecting the roof stability, was taken into account. The simulations used three different horizontal stress ratios to check its influence on the time-dependent roof stability. Due to the change in the horizontal stress, different failure conditions occur at equilibrium condition. More failure occurs with the increase in the maximum horizontal stress. Although the stress distribution does not change significantly, there is a pronounced increase in the stress magnitude. The increase in failure and stress magnitude at equilibrium significantly increases the failed elements within the mine roof with time.

Finally, this study used a step-wise simulation method to achieve a realistic stress path that the mine roof may experience. Dividing the continuous excavation of the mine entry into steps, the model ran under creep condition for the corresponding time after the model run to equilibrium condition of each mining step. The simulation results demonstrate a different failure process. With step-wise excavation, the mine roof experiences an increase in stress when the face moves toward the monitoring location, and then gradually experiences a release of confinement of the underlying coal seam after the face passes. The failure initiating from the right upper corner gradually propagates upward. After reaching the sandstone strata, it starts to propagate horizontally. The distribution of the deformation changes along with the failure propagation within the mine roof. The monitored roof convergence demonstrates that the roof convergence rate gradually increases when the mining face moves closer and decreases after the face passes. The instantaneous and time-dependent deformation mainly occurs in the few mining steps after the mining face passes. 


\section{CHAPTER 8 \\ NUMERICAL STUDY ON THE TIME-DEPENDENT BEHAVIOR OF BEDDED ROOF FOR UNDERGROUND OPENINGS}

\subsection{INTRODUCTION}

A common problem of the mine roof in Pittsburgh coal seam is that the shale rock in the immediate roof is bedded or laminated. The introduction of bedding planes decreases the rock strength and further induces more failure within the mine roof. The presence of bedding planes also induces anisotropic properties of the rock, leading to different mechanical properties of the rock in different directions. This in turn alters the stress distribution within the mine roof and affects the failure mode. Even the mechanical properties of the bedding planes affect the failure mode of the mine roof (Esterhuizen and Bajpayee, 2012). The timedependent sliding can also occur along the bedding planes, which is excluded from this study. However, the numerical study on the time-dependent failure process of massive roof shows that the viscous deformation and strength degradation is very sensitive to the stress distribution and the failed zone within the mine roof. Special attention should be paid to the influence of the bedding planes on stress distribution and failure mode when studying the time-dependent response of bedded or laminated mine roof. The purpose of this chapter is to investigate the time-dependent response of bedded mine roof by explicitly introducing bedding planes into the immediate roof in numerical simulations.

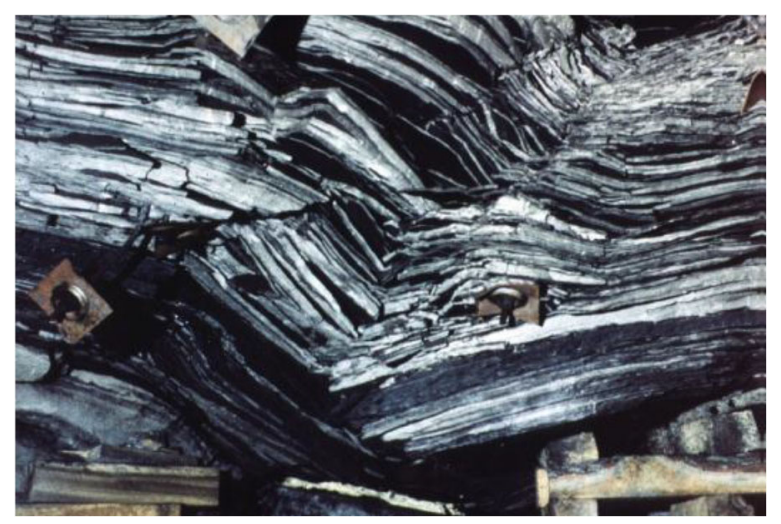

Fig. 8.1. Cutter failure in bedded roof (Esterhuizen and Bajpayee, 2012)

First, a simplified two-dimensional beam model is used to investigate the influence of bedding planes on the stress distribution within the mine roof, as well as the time-dependent response of bedded roof. The simulation of massive mine roof demonstrates that the time-dependent behavior is highly dependent upon the stress distribution. Since the presence of bedding planes alters the stress distribution of the immediate roof, it is necessary to conduct detailed stress analysis to determine the influence of bedding planes. This study used a simplified two-dimensional beam model to demonstrate the stress distribution within bedded rock. In addition, the failure within the immediate roof induces stress redistribution. In order to prevent the stress disturbance by failure, a viscoelastic model was used for the analysis of stress distribution. Areas with high stress concentration can be identified as the places that failure initiates, and the distribution of 
stress will show the potential failure path.

In addition, three-dimensional mode is necessary for the numerical simulations with asymmetric stress conditions. The simplified two-dimensional beam model helps to understand the influence of bedding planes on stress distribution and potential failure behavior of bedded mine roof under symmetric stress condition. However, due to the directional horizontal stress, the stress applied onto the cross-section of the mine entry is not symmetric. The asymmetric stress condition cannot be simulated with the two-dimensional model. This is the drawback of the two-dimensional model. The directional horizontal stress is common in underground coalmines and is a recognized key factor in inducing cutter failure. Therefore, threedimensional model with directional horizontal stress is required to understand the time-dependent response of bedded mine roof to asymmetric stress. This chapter uses the proposed viscoelastic-strain-softening model to represent the intact rocks between bedding planes and time-independent joint model to represent the bedding plane; this will investigate the overall time-dependent response of the intact rock and bedding planes.

In summary, this chapter used both two-dimensional model and three-dimensional model to understand the overall influence of bedding plane on the time-dependent response of mine roof. A simplified beam model was used to study the influence of bedding plane on the stress distribution and on the potential failure of beams under two-dimensional conditions. A three-dimensional mine model with bedded immediate roof was generated to investigate the time-dependent response of mine roof under asymmetric stress conditions. Various factors potentially affecting the response of bedded roof were taken into consideration.

\subsection{TWO-DIMENSIONAL BEAM ANALYSIS OF BEDDED ROOF}

Beam theory is a widely method to study the roof deformation and stability of underground structures, using both continuous and discontinuous approaches to analyze roof beams. The continuous method considers the roof as a continuous lamped beam while the discontinuous approaches involve the analysis of a beam with vertical joints, which is normally called Voussoir beam. Because of the complexity of stress condition and geometry, as well as the observation that the lowest stratum of the roof was not loaded by the upper ones by Fayol (1885), the theoretical analysis of beam theory was mostly conducted for a single layer without external vertical load. However, the situation of a bedded mine roof is more complex than the theoretical model. Numerical simulations can study the influence of bedding planes on the timeindependent stress distribution and deformation of bedded roof, as well as on the time-dependent behaviors.

Based on the boundary condition and stress condition of the immediate roof, this study proposed a simplified beam model for the beam analysis. Fig. 8.2 shows the simplified beam model. The left side of the beam is fixed. The left boundary of the beam can move horizontally due to the deformation of the beam after excavation; however, the horizontal movement is very limited, so it is reasonable to assume that the left boundary is fixed. The right side only constrains the horizontal deformation and it can move downward, representing a symmetric condition. Therefore, the simplified beam model only considers half of a beam. This study applied the in-situ horizontal stress onto the beam as the initial condition. Due to the fix of the horizontal displacement on both sides, the horizontal stress remained in the beam even during the following deformation. Then this study applied a vertical stress smaller than the in-situ one uniformly onto the beam 
to represent the vertical stress transferred from the above strata.

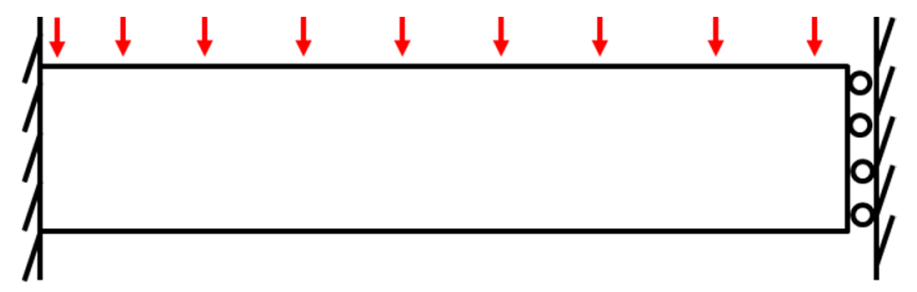

Fig. 8.2. Simplified beam model

\subsubsection{Time-independent and time-dependent behaviors of single intact beam}

The numerical model shown in Fig. 7.1 shows the typical lithology in Pittsburgh coal seam. When one bedding plane is inserted between the immediate shale roof and the main sandstone roof, the immediate shale roof can be considered as a single intact beam. The following viscoelastic stress analysis can help understand the time-independent and time-dependent response of the massive mine roof in Chapter 7.

(1) Time-independent behavior of single intact beam

The simulation with simplified beam model involved a stratum with a half-length of $3.0 \mathrm{~m}$ and a thickness of $1.6 \mathrm{~m}$. The simulation applied an initial stress of $3.6 \mathrm{MPa}$ in the horizontal direction and 1.9 $\mathrm{MPa}$ in the vertical direction into the model and applied a vertical stress of $0.45 \mathrm{MPa}$ from the top of the beam as the boundary condition. Fig. 8.3 plots the distribution of principal stress.
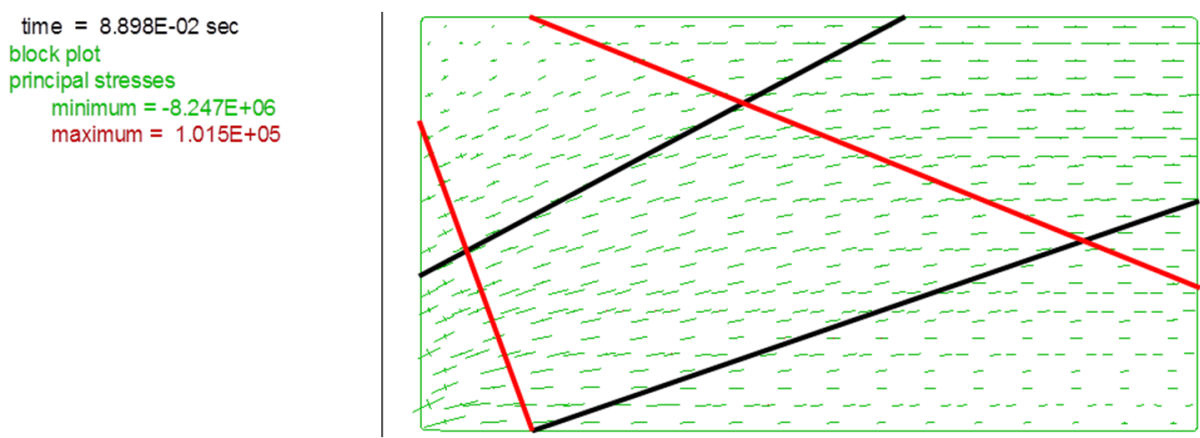

Fig. 8.3. Plot of principal stress for the intact single beam at equilibrium (unit: $\mathrm{Pa}$ )

High compressive stress exists in the area from the left bottom corner to the upper right corner of the stratum. This can lead to the formation of an arch-shaped compressive zone in a full-length beam to sustain the vertical stress, which can be better shown by the major principal stress contour in Fig. 8.4. This archshaped compressive zone can help the rock stratum resist the vertical stress, which can then reduce the vertical stress transferred to the underlying stratum. At the same time, the major principal stress at the left upper corner and at the right lower corner is much smaller than that of the arch-shaped compressive zone; this is the result of beam bending. The vertical boundary stress can induce a clockwise moment in the beam, resulting the bending of the beam. However, the right side is horizontally fixed and can only move in the vertical direction. As a result, the right-side boundary resists the bending of the beam. Compressive stress generates at the upper half section of the right-side boundary and tensile stress generates at the lower part of the right-side boundary. In addition, the left-side boundary of the beam is totally fixed. The bending 
moment of the beam generates tensile stress at the upper half section of the left-side boundary and generates compressive stress at the lower part of the left-side boundary. The induced stress by the beam bending interacts with the initial stress inside the beam leading to the stress distribution in Figs. 8.3 and 8.4. The induced compressive stress at the right upper corner and the left bottom corner increases the total compressive stress at these two sections; they then connect to form the left half of the arch-shaped high compressive zone. At the same time, the induced tensile stress at the upper left corner and right bottom corner offsets the initial compressive stress and decreases the compressive stress. Tensile stress zones can also generate at these two locations.
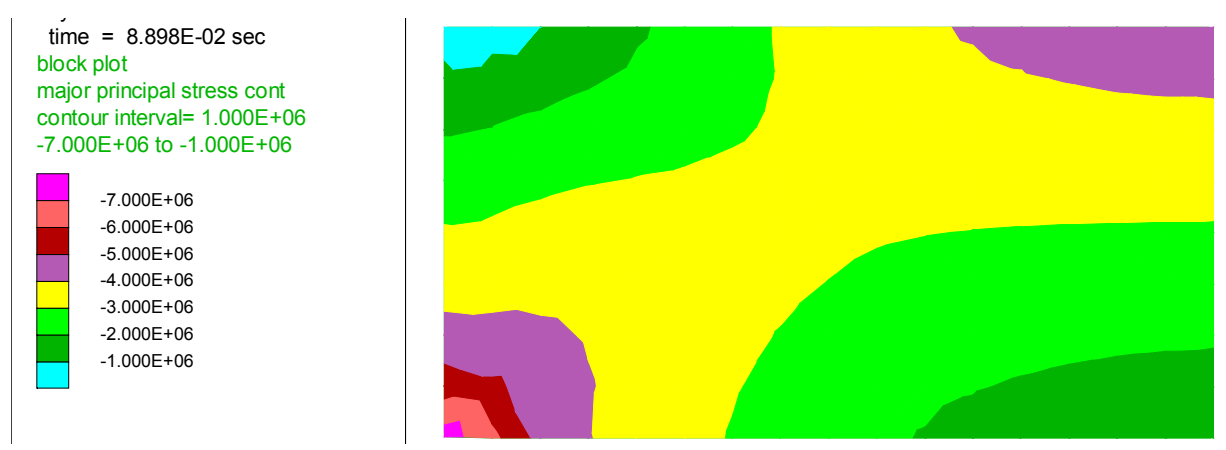

Fig. 8.4. Plot of major principal stress for the intact single beam at equilibrium (unit: Pa)

High horizontal stress is an important factor of cutter roof failure in underground coalmines. Stress analysis with a beam model can assist in illustrating the stress distribution within the roof beam and the influence of horizontal stress. The simulations recreated four situations with the simplified beam model. The first model has one beam with only a vertical stress of $0.45 \mathrm{MPa}$ without initial stress; the other three models have a vertical stress of $0.45 \mathrm{MPa}$ and initial stress where the vertical stresses are all $1.9 \mathrm{MPa}$ and the horizontal stresses are 1.9 MPa, 3.6 MPa, and 5.4 MPa, respectively. The following four figures show the simulation results.
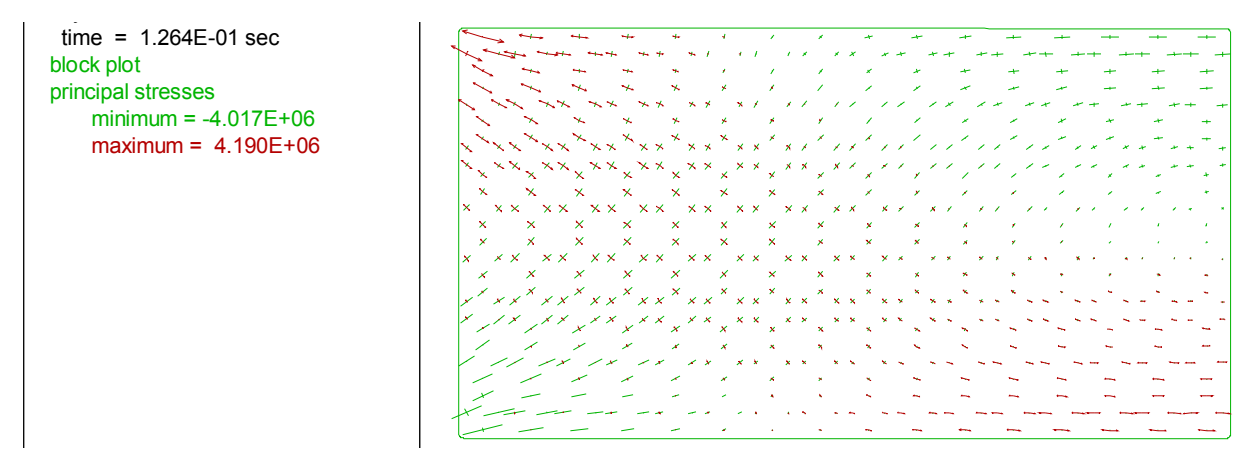

Fig. 8.5. Plot of major principal stress for the intact single beam at equilibrium when only vertical boundary stress is applied (unit: $\mathrm{Pa}$ )

Fig. 8.5 illustrates the stress distribution resulting from the vertical stress of $0.45 \mathrm{MPa}$. There was no initial stress within the beam; the stress distribution is the result of applied vertical stress, which generated bending moment in the beam. Two corners generated high compressive stress and the other two had high tensile stress. The two corners with high compressive stress connected to form half of an arch-shaped compressive zone to resist the beam bending. A vertical stress of $0.45 \mathrm{MPa}$ can lead to compressive principal 
stress as large as 4.0 $\mathrm{MPa}$ and tensile principal stress as large as $4.2 \mathrm{MPa}$, indicating that the stress distribution within the beam can be highly dependent on the vertical stress transferred from the above rock layer or beam. In addition, the generated stress from the applied vertical stress can interact with the initial stress leading to the stress distribution shown in Fig. 8.3.
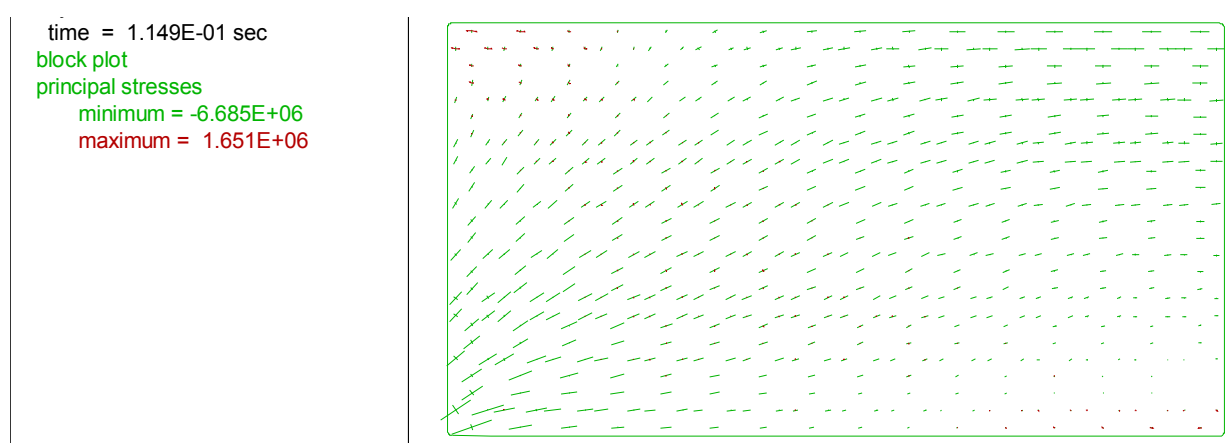

Fig. 8.6. Plot of major principal stress for the intact single beam at equilibrium when vertical boundary stress and 1.9 MPa of initial horizontal stress is applied (unit: $\mathrm{Pa}$ )
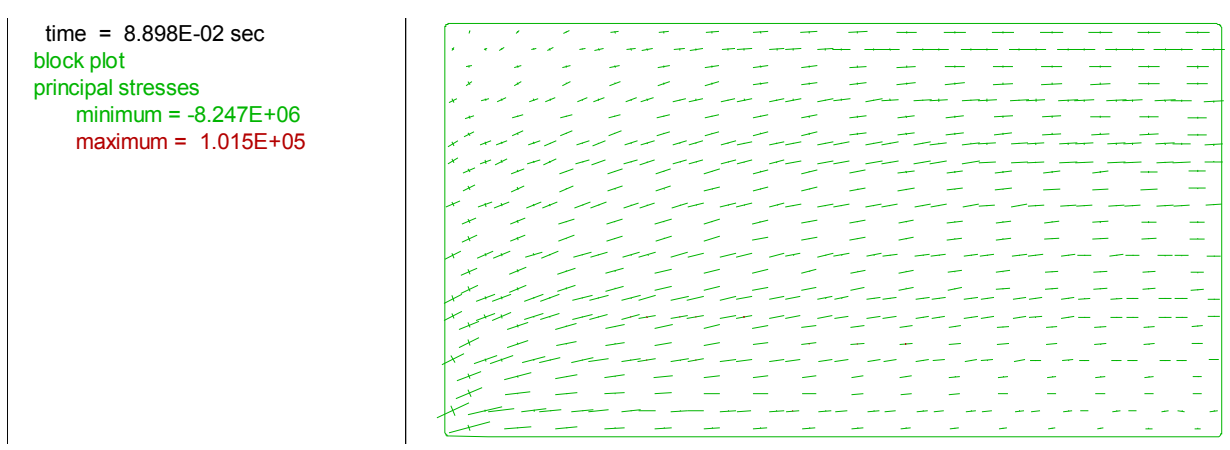

Fig. 8.7. Plot of major principal stress for the intact single beam at equilibrium when vertical boundary stress and 3.6 MPa of initial horizontal stress is applied (unit: $\mathrm{Pa}$ )
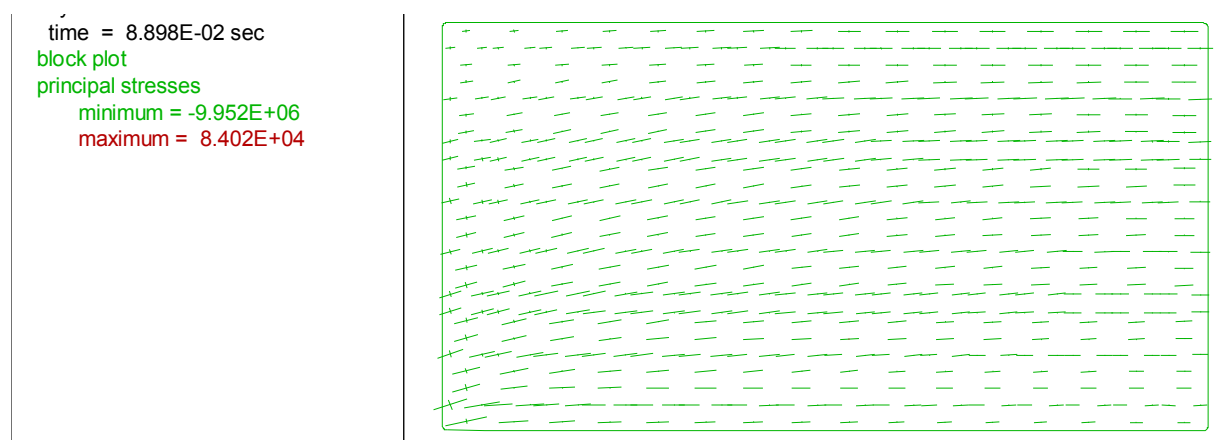

Fig. 8.8. Plot of major principal stress for the intact single beam at equilibrium when vertical boundary stress and 5.4 $\mathrm{MPa}$ of initial horizontal stress is applied (unit: $\mathrm{Pa}$ )

In addition, comparing the principal stress distribution in Fig. 8.5 through Fig. 8.8 shows the influence of horizontal stress. From Fig. 8.5 to Fig. 8.8, a constant vertical boundary stress was applied, and the initial horizontal stress gradually increased. We can find that the magnitude of the tensile principal stress gradually decreased while the magnitude of the compressive principal stress gradually increased. The principal stress of some areas changed from tension to compression, which can inhibit the tensile failure within the roof beams and increase roof stability. However, the increasing compressive principal stress can induce shear 
failure within the roof at the entry corner. Therefore, if an entry is mined within a rock stratum with low horizontal stress, tensile stress concentration can occur at midspan potentially leading to tensile failure; high horizontal stress in the strata can induce arch-shaped high compressive zone, especially at the entry corners, potentially inducing shear failure.

Furthermore, the increasing horizontal stress changed the direction of the principal stress within the beam; comparing the direction of major principal stress at the area close to the left bottom corner confirms this phenomenon. With increasing initial horizontal stress, the direction of the major principal stress is closer to the horizontal direction from Fig. 8.5 to Fig. 8.8, indicating that the influence of the stress induced by beam bending becomes less and less significant as the horizontal stress increases. The change in the direction of major principal stress can be relative to the influence of horizontal stress on cutter roof failure. Aggson (1979) and Su and Peng (1987) found that the angle of propagation of the failure surface is dependent on the stress ratio of horizontal stress to vertical stress, and that lower angles relative to the horizontal direction are usually due to a higher stress ratio.

From the above stress analysis, we can see that shear failure usually initiates from the entry corner due to the high stress concentration. The failure then propagates along the compression arch, where there is high major principal stress and low minor principal stress. The initial horizontal stress can offset the tensile stress at the entry center resulted from beam bending and, at the same time, increase the severity of the shear stress concentration at the entry corner.

(2) Time-dependent behavior of single intact beam

Creep simulation of the single intact beam ran for some time after analyzing the stress distribution at equilibrium. This study then analyzed the evolution of stress distribution with time. The following figures show the plots of major principal stress at equilibrium, after one day, and after one week.
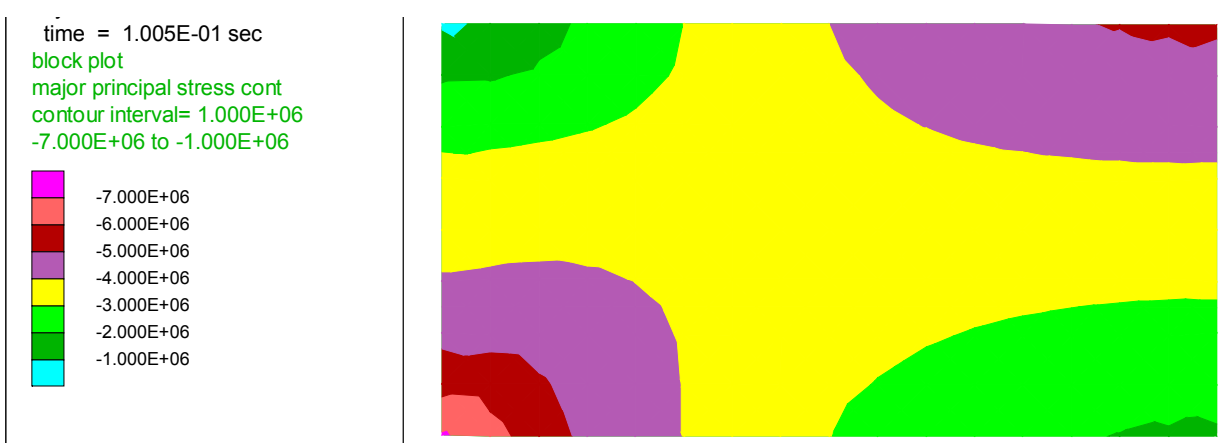

Fig. 8.9. Plot of major principal stress for single intact beam at equilibrium (unit: Pa) 


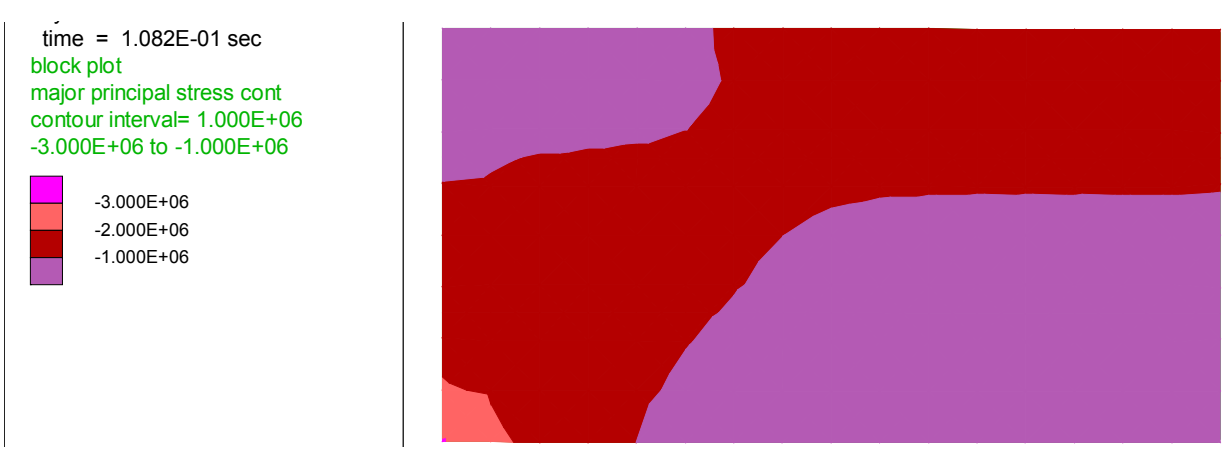

Fig. 8.10. Plot of major principal stress for single intact beam after one day (unit: Pa)

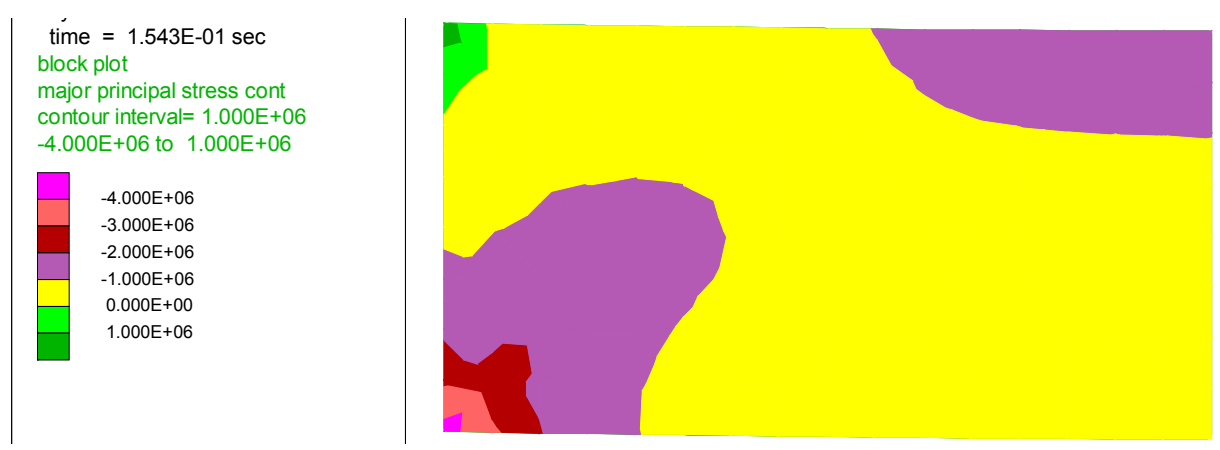

Fig. 8.11. Plot of major principal stress for single intact beam after one week (unit: Pa)

Comparing these three figures shows that the magnitude of the major principal stress gradually decreased with time in most parts of the beam. The stress reduction with time was studied from relaxation point of view. Eq. $3.1 \mathrm{~b}$ represents the stress-strain relation for Burgers material in two parts: the volumetric part and deviatoric part. $1 / \mathrm{K}$ is the reciprocal of bulk modulus, representing the resistance of the material to volumetric stress. At the same time, the term $\left\{\frac{1}{E_{m}}+\frac{1}{\eta_{m}} t+\frac{1}{E_{k}}\left[1-\exp \left(\frac{-E_{k} t}{\eta_{k}}\right)\right]\right\}$ represents the reciprocal of an apparent shear modulus. With the increase in time, the value of this term increases, indicating the decrease of the apparent shear modulus. The apparent shear modulus represents the resistance of the material to deviatoric stress and the decreasing value indicates that the resistance of the material to deviatoric stress gradually decreases with the accumulation of time. If using the stiffness of a spring to represent the apparent shear modulus, the mechanism of the rock viscous behavior results in the decrease of the spring stiffness and therefore makes the spring softer and softer. The fixed boundary condition in the horizontal direction make the stress gradually relax from the roof beam.

In addition, the decrease in the apparent shear modulus with time made the rock easier to deform. The increasing deformation in turn enlarged the beam bending and led to the increasing tensile principal stress. The connection between the tensile stress zones- the left upper corner and the right bottom corner- broke the compression arch, as shown in Fig. 8.11. Since the compression arch helped to resist the beam bending, the breaking of the compression arch further reduced the resistance of the beam to bending. The breaking of the compression arch and the decreasing apparent shear modulus both increase the potential of beam bending; as a result, there is a significant increase in the displacement of the whole beam from one day to one week. 
Furthermore, the potential failure mode of the single intact beam can be determined from the viscoelastic stress analysis. Along with the bending of the beam with time, Fig. 8.12 shows that compressive stress concentration occurred at the left bottom corner and the right upper corner, and tensile stress concentrated at the left upper corner and the right bottom corner. Extracting the influence of fixed boundary effect, stress concentration still occurs at these areas with increasing beam bending, especially at the left bottom corner representing the entry corner and at the right bottom corner representing the roof midspan. It potentially induces time-dependent shear failure from the entry corner and tensile failure from the midspan.
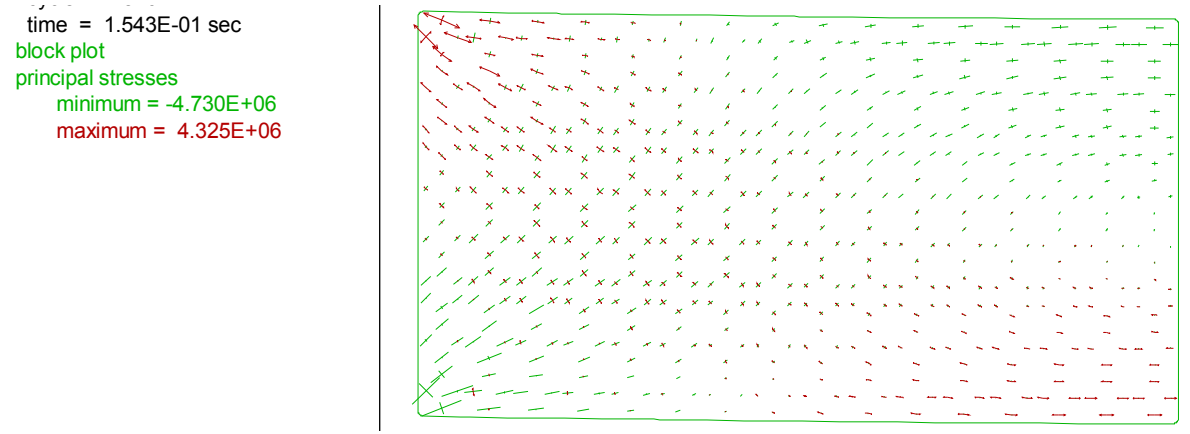

Fig. 8.12. Plot of principal stress for single intact beam after one week (unit: Pa)

The analysis of the time-dependent stress distribution demonstrates that the shear stress concentration at the entry corner and the tensile stress concentration at the midspan is partially a result of the timedependent deformation of the beam. On one hand, installing bolts at the center of the roof can provide a force to balance the vertical load transferred from the above strata and gravity. The bending moment within the beam can significantly reduce. This action can prevent the time-dependent tensile stress at the midspan. On the other hand, installing vertical or inclined bolts close to the entry corner can increase the minor principal stress, and the shear stress close to the entry corner will decrease. Therefore, this will reduce the overall potential for shear failure.

All the above stress analyses are based on elastic models without failure. The elastic model is very useful for stress analyses; however, the real situation is much more complex than the elastic condition. Failure can occur with the stress redistribution after excavation and can further induces stress redistribution.

\subsubsection{Time-independent and time-dependent behaviors of bedded beam}

The simulations inserted different numbers of bedding planes into the beam to investigate the timeindependent and time-dependent behavior of the bedded beam. The bedding planes were first considered as planes without strength, indicating that there was no cohesion, friction angle, and tensile strength assigned to the bedding planes. This study examined the influence of the beam thickness, and the influence of bedding plane strength by assigning different strength parameters.

\section{(1) Influence of beam thickness}

Fig. 8.13 plots the principal stress distribution at equilibrium for the beam with one bedding plane. The comparison with Fig. 8.3 shows that they work as if they are two beams, and the inclusion of one bedding plane completely changes the stress distribution compared to that of an intact beam. In Fig. 8.13, there is 
high compressive zone extending from the left bottom corner to the right upper corner within each of the beam. The left upper corner and the right bottom corner have low compressive stress and even tensile stress in both of the separated beams. Therefore, each layer works like the single intact beam in Fig. 8.3. Due to the decreasing beam thickness from Fig. 8.3 to Fig. 8.13, however, the stress condition becomes worse for roof stability. There is an increase in the maximum principal stress and the minimum principal stress, as well as showing a higher possibility of shear failure and tensile failure in Fig. 8.13 than Fig. 8.3.
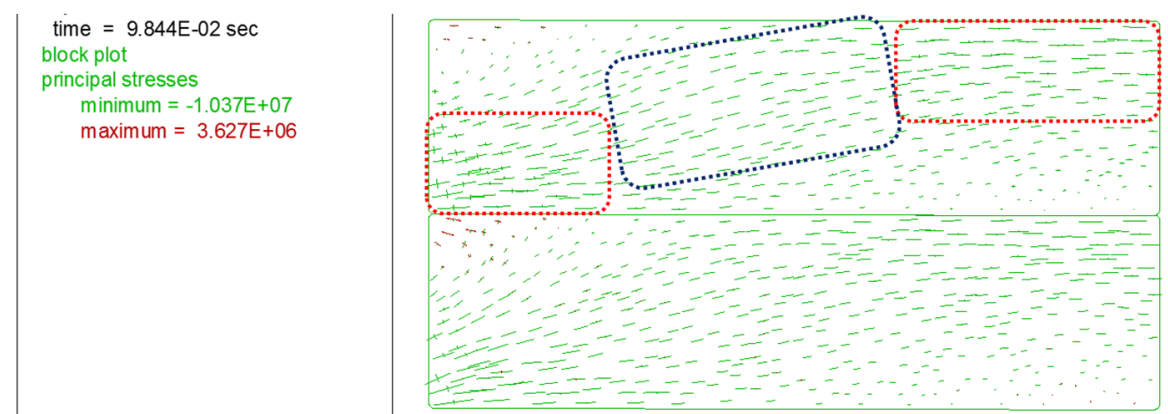

Fig. 8.13. Plot of principal stress for $0.8 \mathrm{~m}$-thick beam at equilibrium (unit: $\mathrm{Pa}$ )

Due to the decrease in the beam thickness, the resistance of each beam to bending decreases significantly. Under the same boundary and loading condition, the decrease in the resistance to bending leads to more bending and displacement, which further deteriorates the stress distribution within the beams. When more bedding planes were inserted into the beam, the condition became worse, as shown in Figs. 8.14 and 8.15. There was a further increase in the maximum principal stress and minimum principal stress. More displacement occurred when the beam thickness decreased, and the bending of the rock layers significantly affected the stress distribution.
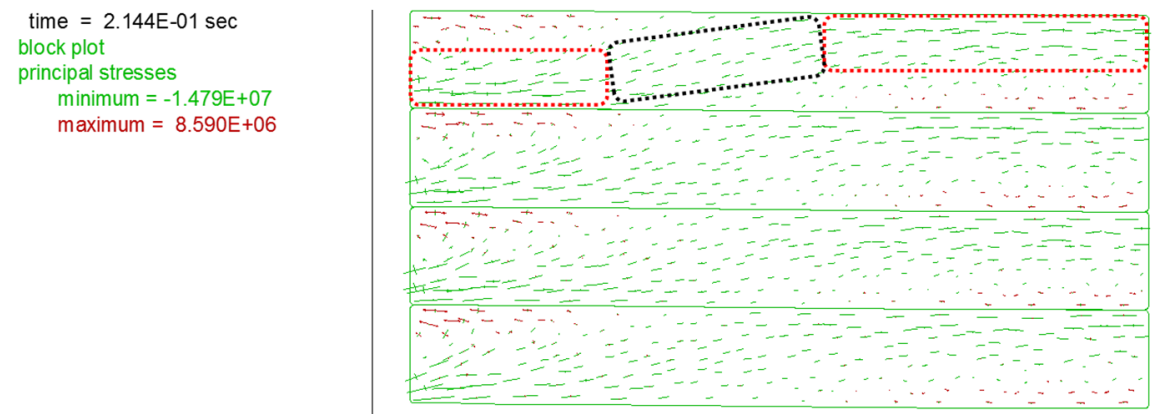

Fig. 8.14. Plot of principal stress for $0.4 \mathrm{~m}$-thick beam at equilibrium (unit: $\mathrm{Pa}$ )
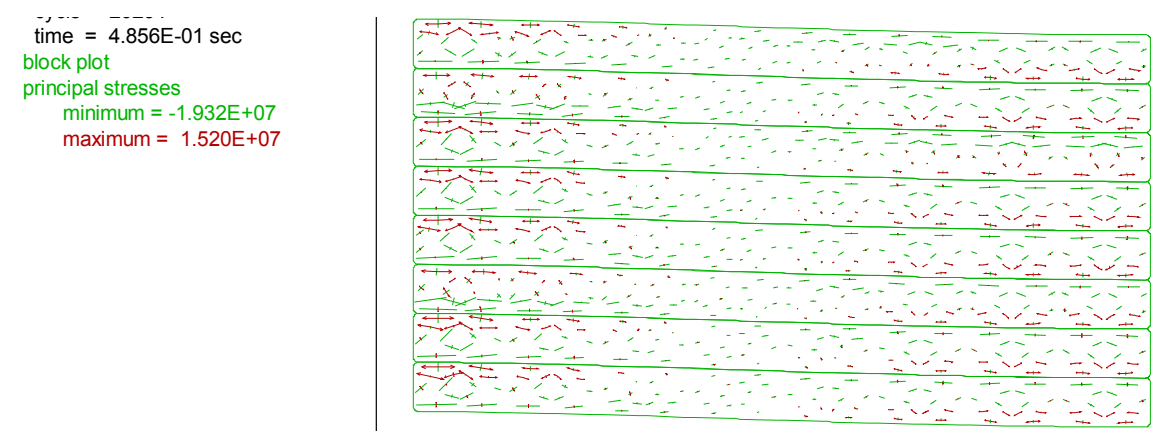

Fig. 8.15. Plot of principal stress for $0.2 \mathrm{~m}$-thick beam at equilibrium (unit: $\mathrm{Pa}$ ) 
Another observation from Fig. 8.13 through Fig. 8.15 is that the compression arch disappears as the beam thickness decreases. In Fig. 8.13, the high compressive zones at the left bottom corner and the right upper corner connect significantly from the inclined principal stress. When the beam thickness decreases to $0.4 \mathrm{~m}$ in Fig. 8.14, the connection/transaction zone is much weaker than the $0.8 \mathrm{~m}$-thick beam. When the beam thickness further reduces to $0.2 \mathrm{~m}$ in Fig. 8.15, there is no apparent connecting area between the two high compressive zones. This could be the result of an increasing slenderness ratio and the induced stress by increasing beam bending. The following two figures illustrate the gradually disappeared compression arch from the distribution of major principal stress.
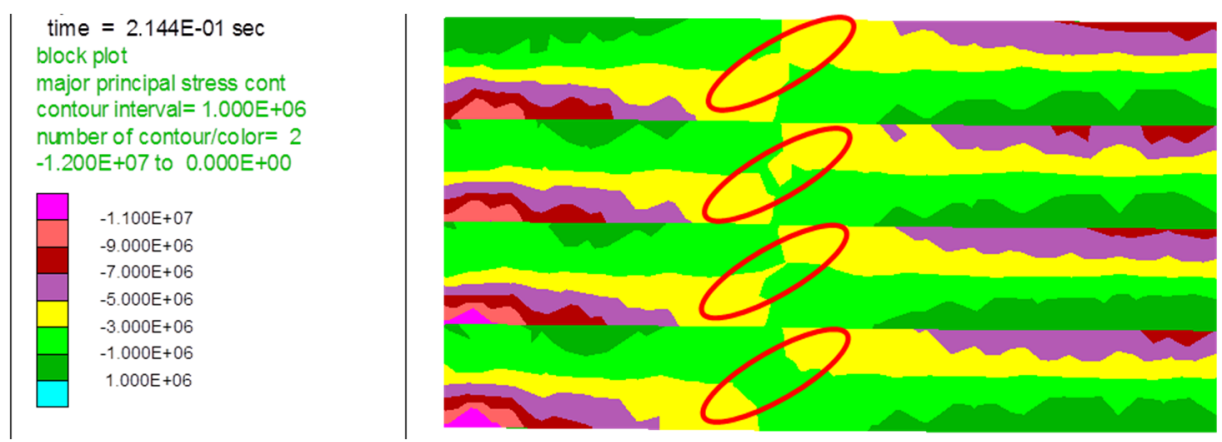

Fig. 8.16. Plot of major principal stress for $0.4 \mathrm{~m}$-thick beam at equilibrium (unit: Pa)
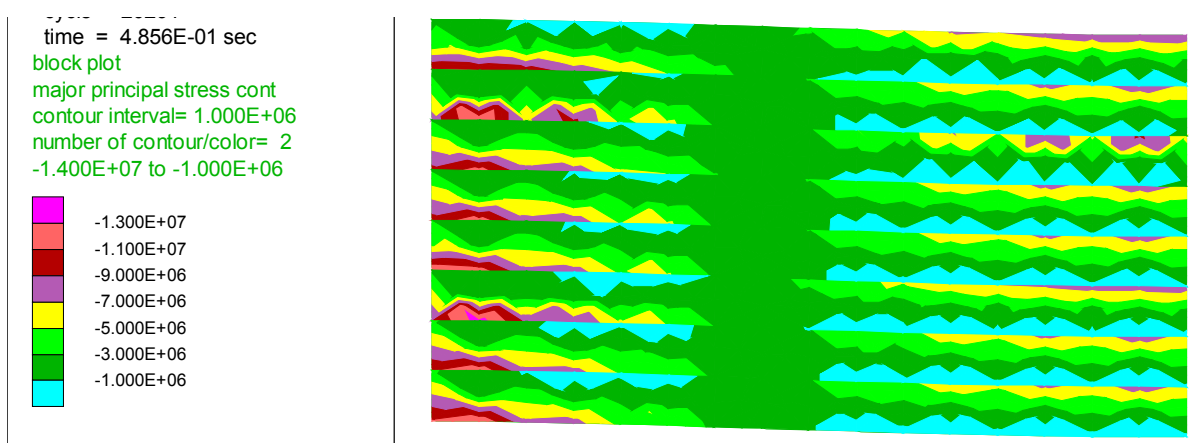

Fig. 8.17. Plot of major principal stress for $0.2 \mathrm{~m}$-thick beam at equilibrium (unit: Pa)

Furthermore, the time-dependent behavior of each rock layer in the bedded beam is similar to that of the single intact beam. For example, Figs. 8.18 and 8.19 plot the major principal stress of the beam with $0.4 \mathrm{~m}-$ thick layers after one day and one week, respectively. From Fig. 8.16 to Fig. 8.18, the stress within the roof relaxes globally. Due to the increase in deformation with time, however, the stress distribution is further affected by the beam bending. From one day to one week, there is an increase in the compressive major principal stress at the left bottom corner of each beam and an increase in the tensile major principal stress at the right bottom corner. This trend is similar to that of a single intact beam. In addition, due to the difference in the constraint, there are differences in the stress distribution between the layers. The bottom layer is free to move downward to the entry and achieve the largest deformation among all these layers; in contrast, the underlying layer provide resistance to the upper layers and, as a result, the deformation gradually decreases from the bottom layer. The influence of the beam bending on the stress distribution decreases correspondingly. Therefore, the bottom layer deforms the most to the entry and experiences the worst condition. When the bottom layer fails, the layer directly above it may then achieve more deformation 
and deteriorate stress condition.

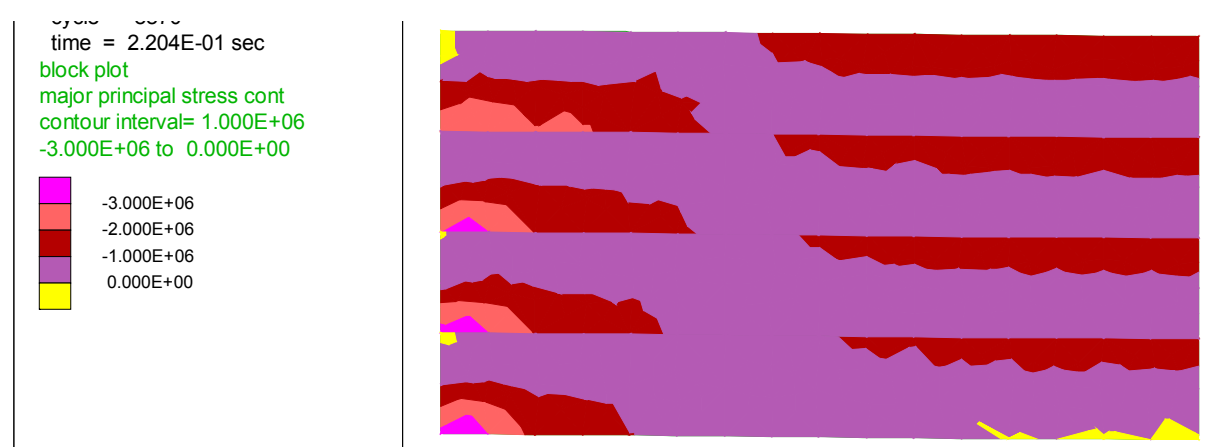

Fig. 8. 18. Plot of major principal stress for $0.4 \mathrm{~m}$-thick beam at equilibrium (unit: Pa)
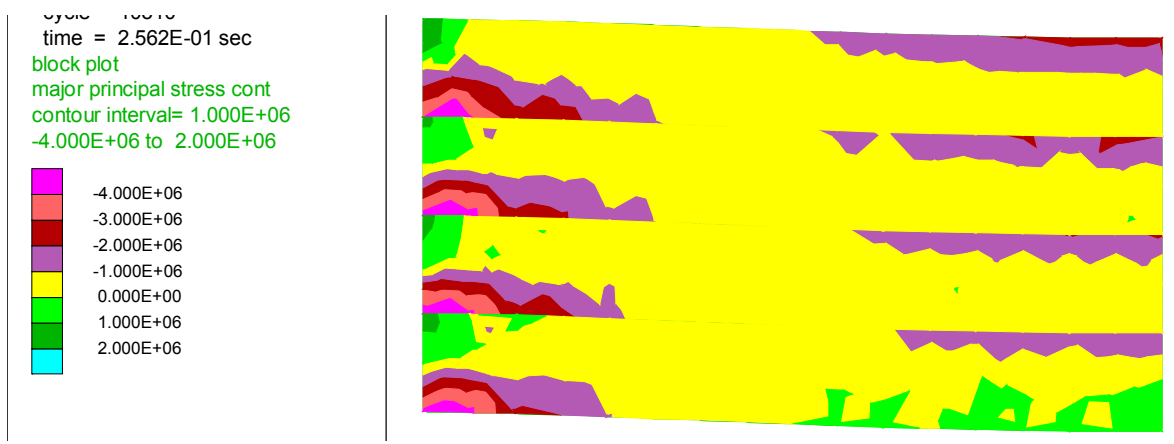

Fig. 8.19. Plot of major principal stress for $0.2 \mathrm{~m}$-thick beam at equilibrium (unit: $\mathrm{Pa}$ )

(2) Influence of bedding plane strength

The above analysis ignored the strength of the bedding planes; as such, this results in the two surfaces surrounding one bedding plane being unable to transfer shear stress between the adjacent two layers. The bedding plane cannot sustain any tensile stress. The only stress that can transfer between two layers is the compressive stress in the perpendicular direction. Therefore, each of the layers behaves like a separated beam. However, the situations are different if the layers are bonded together.

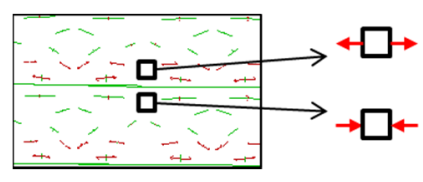

Fig. 8.20. Stress analysis between one bedding plane without strength

Fig. 8.20 shows an enlarged view of part of Fig. 8.15, describing an area surrounding part of the bedding plane close to the left edge. It is clear that either layer does not restrict the stress within the two layers. There is high stress difference across the bedding plane: one with tensile stress, and the other with compressive stress. If there is a bonded bedding plane with relative displacement between these two layers, shear stress will induce along the bedding plane and further affect the stress distribution of the two sides of the bedding plane. As a result, the magnitude of the tensile stress and the compressive stress on both sides can decrease.

The following simulations used three different cohesion values, which were considered to be low, 
medium and high. The distribution of major principal stress for $0.8 \mathrm{~m}$-thick beam with different strengths was the chosen example, plotted in Fig. 8.21 through Fig. 8.23. The first pronounced change with the increasing bedding plane strength is the movement of the areas with low compressive stress at the left upper corner of the low beam and with high compressive stress at the left bottom corner of the upper beam. It was likely that there was shear stress built within the bedding plane, affecting the stress distribution within the surrounding two layers. Fig. 8.24 plots the shear stress along the bedding plane with different strengths. Here, the peak shear stress gradually increases and moves outward from the left edge with increasing bedding plane strength. There is no shear stress in most parts of the bedding planes. The investigation of the displacement along bedding planes shows that the areas surrounding the bedding plane without shear stress are deforming consistently without relative shear displacement. Therefore, the relative shear displacement in the part of bedding plane above the entry corner and the induced shear stress in the bedding plane affects the distribution of major principal stress.
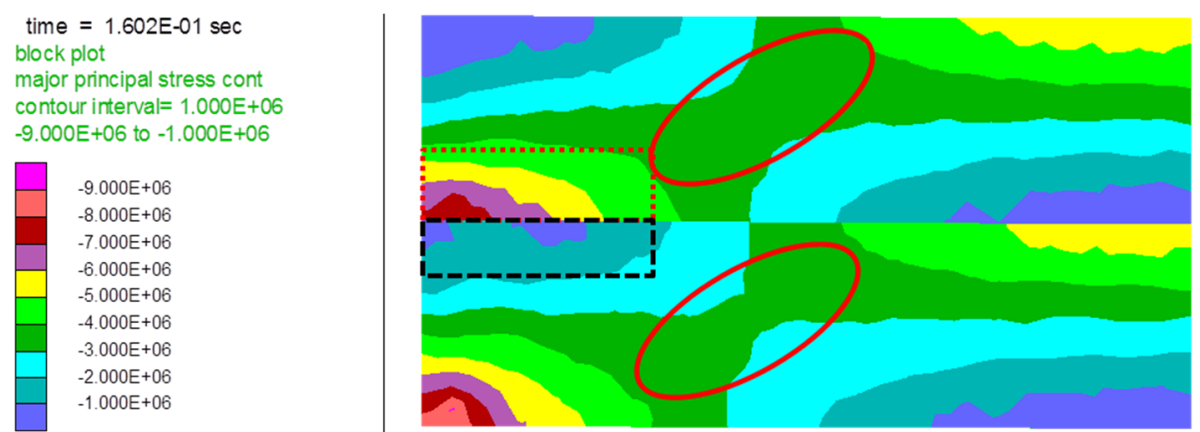

Fig. 8.21. Plot of major principal stress for $0.8 \mathrm{~m}$-thick beam with low bedding plane strength at equilibrium (unit:

$\mathrm{Pa})$
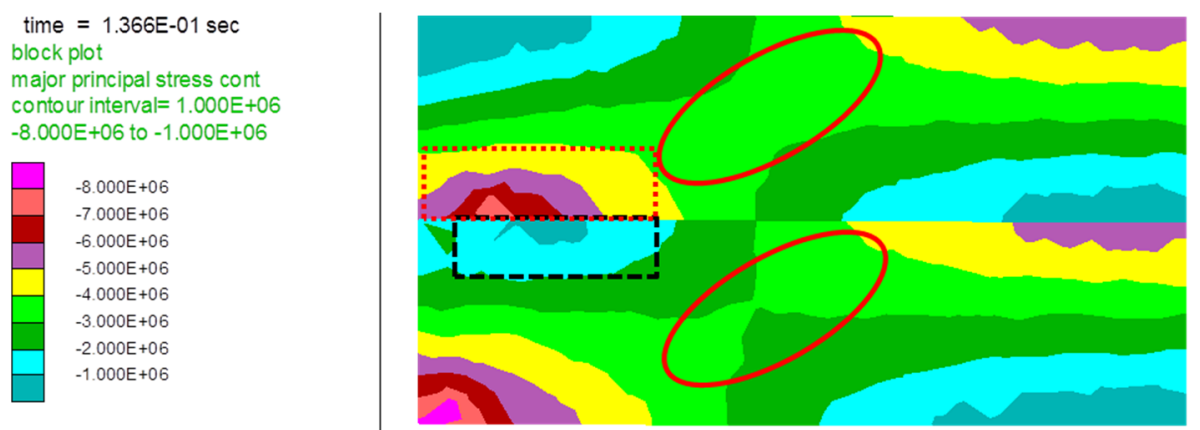

Fig. 8.22. Plot of major principal stress for $0.8 \mathrm{~m}$-thick beam with medium bedding plane strength at equilibrium (unit: $\mathrm{Pa}$ )
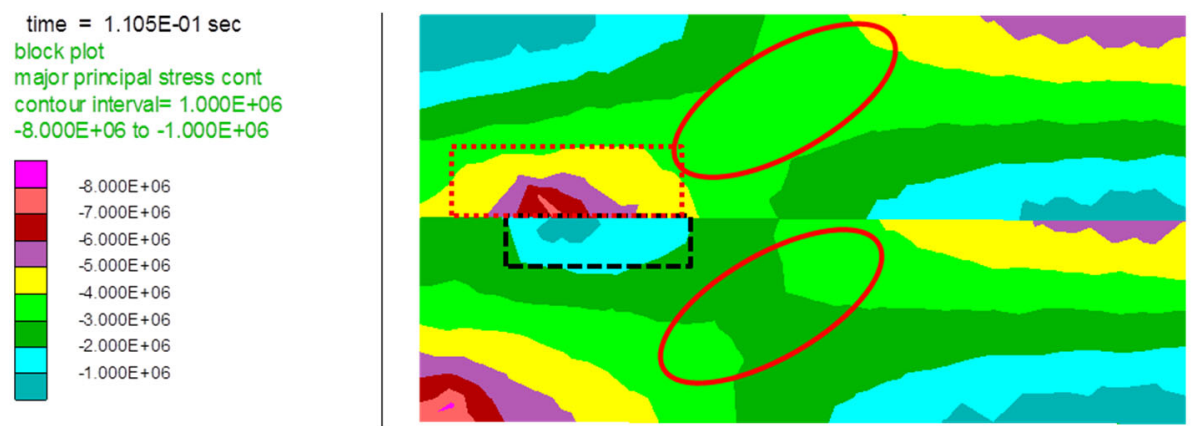

Fig. 8.23. Plot of major principal stress for $0.8 \mathrm{~m}$-thick beam with high bedding plane strength at equilibrium (unit: 


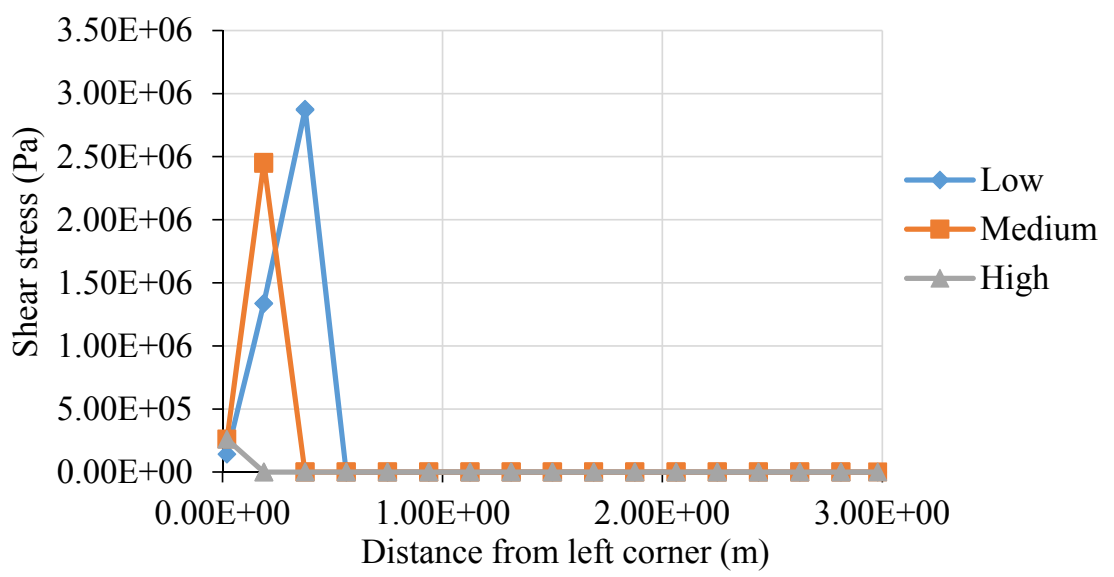

Fig. 8.24. Plot of shear stress within bedding plane with different strengths

In addition, the movement of the stress zone at the left corners affects the stress distribution within each layer. On one hand, the connection between the two high compression zones in the low layer gradually reduces with the movement of the low principal stress area from the left edge, a result of the increasing bedding plane strength shown in Fig. 8.21 through Fig. 8.23. The low principal stress zones at the left upper corner and at the right bottom corner are closer and make the compression arch narrower. When the compression arch is broken, the resistance of the layer to bending will reduce. This is an unsafe condition for the low layer. On the other hand, the connection between the two high compression zones in the upper layer enhances with the movement of the high principal stress area from the left edge. The increase in the bedding plane strength makes the two high compressive zones closer, and the compression arch becomes thicker.

Finally, the time-dependent behavior of the bedded beam with bedding plane strength is similar to that of beams without bedding plane strength. The only difference lies in the locations where high principal stress occurs.

\subsection{TIME-DEPENDENT SIMULATION OF BEDDED MINE ROOF}

Two-dimensional viscoelastic simulations in Section 8.1 provides insight into the influence of bedding planes on the time-dependent roof stability through the stress distribution and potential failure path for the models with different numbers and strengths of bedding plane. However, the real situation is much more complex than the two-dimensional model. One of the key factors in studying these influences is the directional horizontal stress, which is critical in cutter roof failure and cannot be simulated in the twodimensional model. Due to the directional horizontal stress, the boundary condition of the model will no longer be symmetric, causing the model to be a three-dimensional problem. In addition, the simplified beam analysis only considers the viscoelastic situation and is only for the stress analysis. This did not include failure analysis. Once failure occurs within the elements, extra load transfers to adjacent elements and can potentially induce more failure, making the analysis of stress distribution to be far more difficult. 


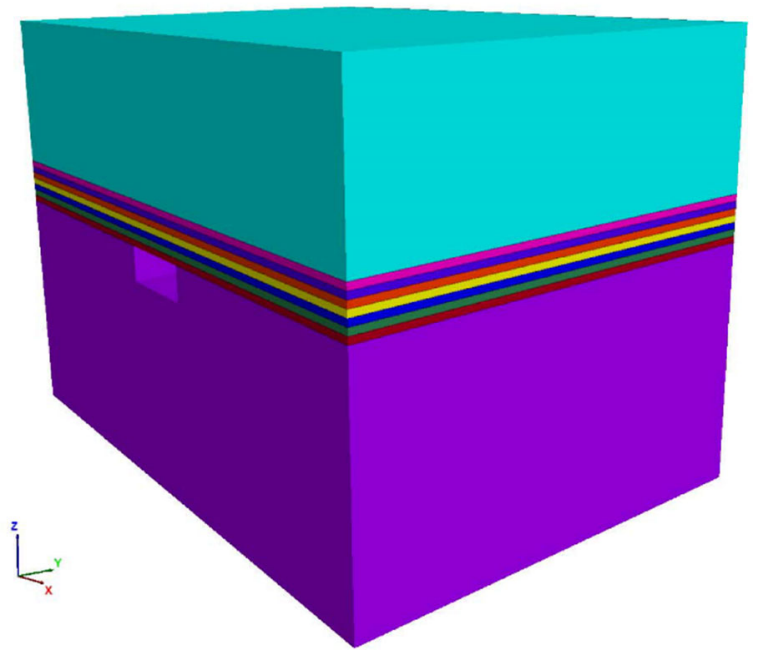

Fig. 8.25. Mine model with bedded roof

The simulations in this section used a three-dimensional mine model with bedded roof, shown in Fig. 8.25. Compared with the model in Chapter 7, the immediate shale roof in this model inserted more bedded plane into the roof. All the other parameters are the same as the model in Chapter 7.

\subsubsection{Time-dependent response of bedded mine roof}

(1) Immediate roof without bedding plane

As mentioned earlier, the shale immediate roof is similar to the single intact beam in Section 8.1 when one bedding plane was inserted between the shale immediate roof and the sandstone main roof. It can be considered as a special case of the bedded mine roof. However, the analysis of single intact beam was based on the symmetric stress condition, which is different from the true situation. Therefore, this study analyzed the simulation results of the massive roof in Chapter 7 to obtain the time-dependent response of the single intact beam.

Fig. 8.26 shows the distribution of major principal stress at equilibrium. There are similarities in the stress distribution between the mine model and the beam model. It is notable that there is an arch-shaped zone with high major principal stress within the immediate roof. Due to the thickness of the immediate roof, a compression arch forms to resist the bending of the roof. The appearance of the compression arch in the mine model is similar to the response of the simplified beam model with single intact beam. However, due to the directional horizontal stress, the high compressive zone is not symmetric under three-dimensional condition. The major principal stress on the right side of the entry is generally larger than the left side. The stress distribution in Fig. 8.26 shows that the direction of the major principal stress on the right side is steeper than that on the left.

In addition, there is one area with low major principal stress, located at the midspan of the whole beam. This is similar to the beam analysis, where the low major principal stress exists at the right bottom area of the half beam. However, there is no area with low major principal stress existing in the mine model corresponding to the left upper area of the simplified beam model; this is due to the assumed boundary conditions in the simplified beam model. 


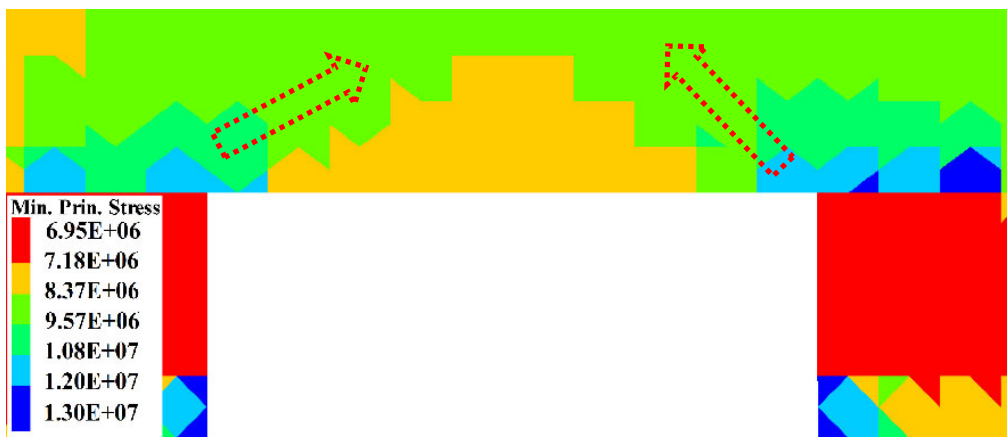

Fig. 8.26. Plot of major principal stress distribution at equilibrium for the model with massive roof (unit: $\mathrm{Pa}$ )

Fig. 8.27 plots the distribution of maximum shear stress at equilibrium. There is an arch-shaped zone within the immediate roof; however, this is different from the compression arch shown in Fig. 8.26. Maximum shear stress represents the difference between major principal stress and minor principal stress. Aside from major principal stress, minor principal stress affects the distribution of maximum shear stress. As marked on the figure, high major principal stress and low minor principal stress are likely to occur within the arch-shaped zone of maximum shear stress in Fig. 8.27. As the developed model used Mohr-Coulomb failure criterion, based on shear failure, to predict failure, the failure of the roof rocks potentially locates within the arch zone with high maximum shear stress. Comparing Figs. 7.14 and 8.27 confirms this, as the failure occurs along the arch-shaped zone shown in Fig. 8.27.

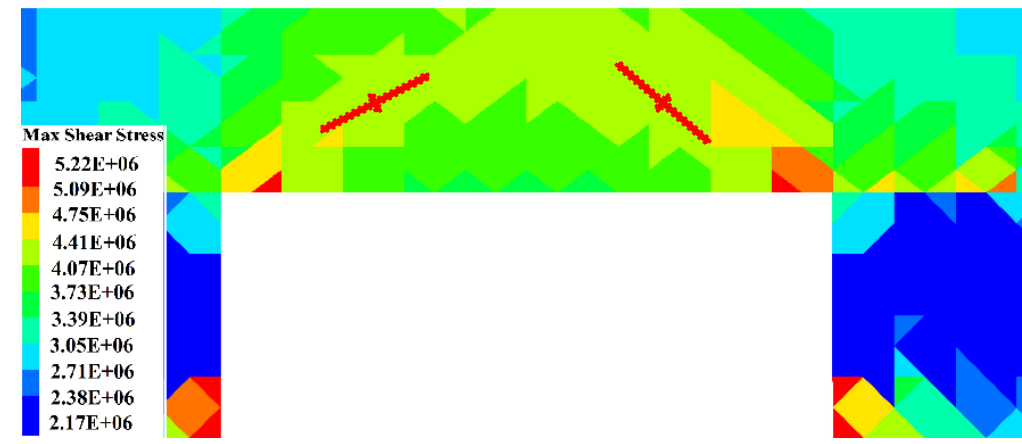

Fig. 8.27. Plot of maximum shear stress distribution at equilibrium for the model with massive roof (unit: Pa)

(2) Immediate roof with bedding planes

Different stress distributions and failure profiles are notable between massive mine roof and bedded mine roof. When the immediate roof is massive or intact, the failure initiates from the entry corners where stress concentration occurs, as shown in Fig. 7.7. For bedded roof, there is shear failure initiating in the first layer of the roof rock, shown in Fig. 8.28. However, more failure occurs at the center of the roof above the first layer. Fig. 8.28 illustrates that the joint normal stress within the failed central area is much lower than in other areas; this indicates that there is a relative opening or deformation between these rock layers, which reduces the confinement to the upper layers. With the deformation of the rock layers, the bending of the beam generates tensile stress, further decreasing the compressive stress within these layers. As a result, the failure initiates from the central area of the roof. The comparison of the failure profiles at equilibrium in Figs. 7.7 and 8.28 shows that far more failure has induced in the bedded roof than in the massive roof. Therefore, the existence of the bedding plane changes the failure profile of the mine roof. 


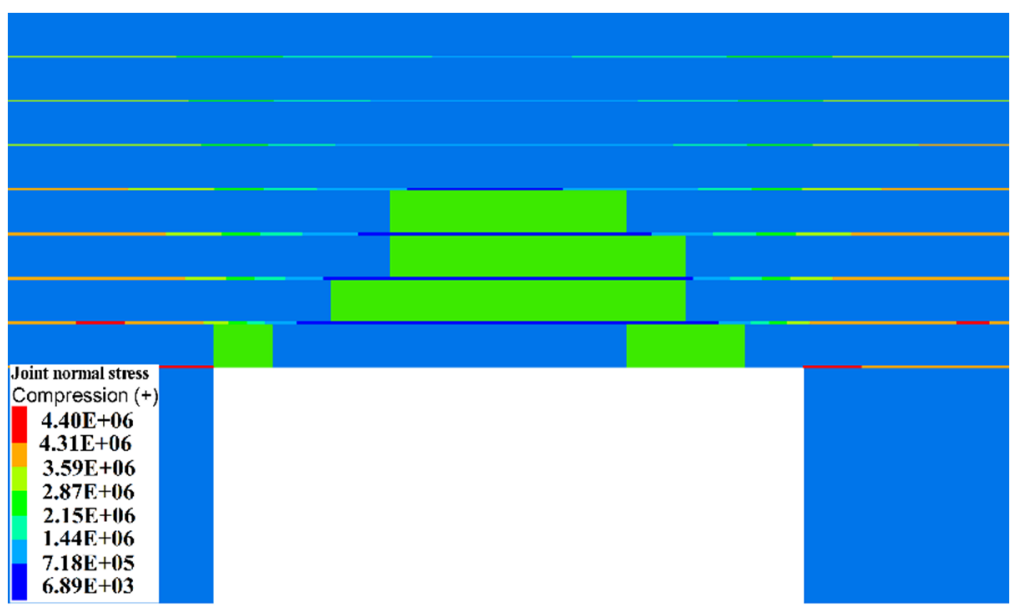

Fig. 8.28. Plot of failure condition and joint normal stress magnitude at equilibrium for the model with bedded roof (unit: $\mathrm{Pa}$ )

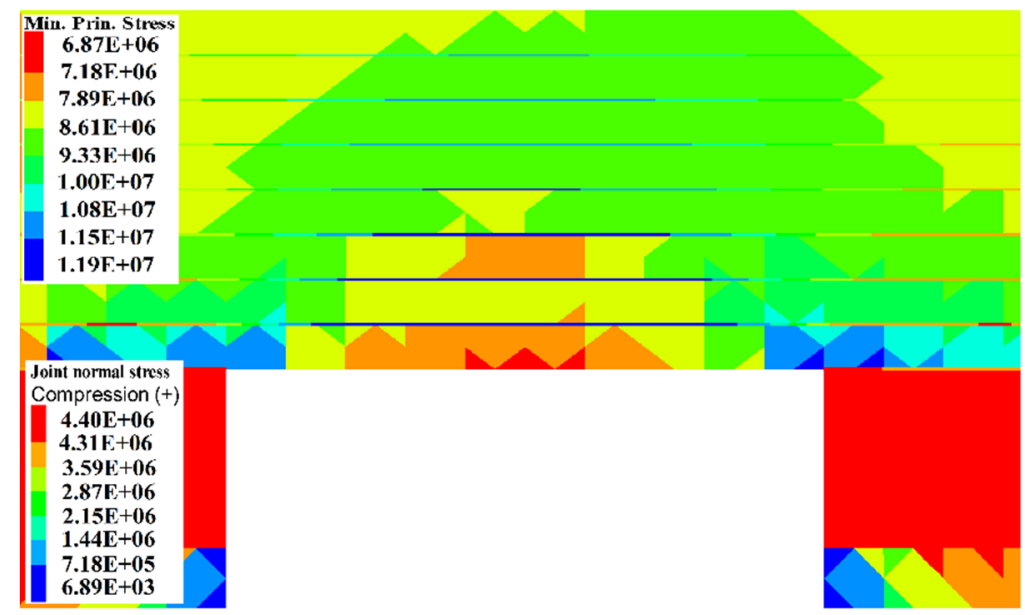

Fig. 8.29. Plot of major principal stress and joint normal stress magnitude at equilibrium for the model with bedded roof (unit: $\mathrm{Pa}$ )

Corresponding to the joint normal stress distribution, there is low principal stress within an area directly above the mine entry, as shown in Fig. 8.29. The reducing beam thickness prevents the formation of compression arch in each rock layer. However, an arch-shaped compression zone forms within the whole immediate roof. Due to the increasing deformation of the bedded roof, the stress transfers into deep roof rocks. In addition, the compression arch shows the influence of directional horizontal stress on the stress distribution. The thickness of the compression arch on the right side is much larger than the left side. However, the distribution of joint normal stress shows no influence, being almost symmetric. 


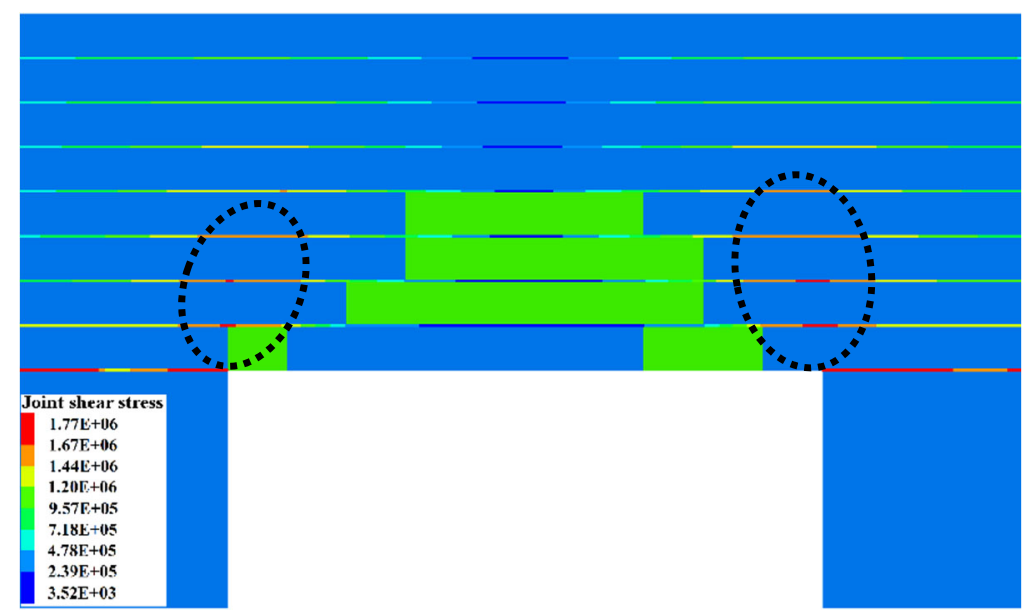

Fig. 8.30. Plot of failure condition and joint shear stress magnitude at equilibrium for the model with bedded roof (unit: Pa)

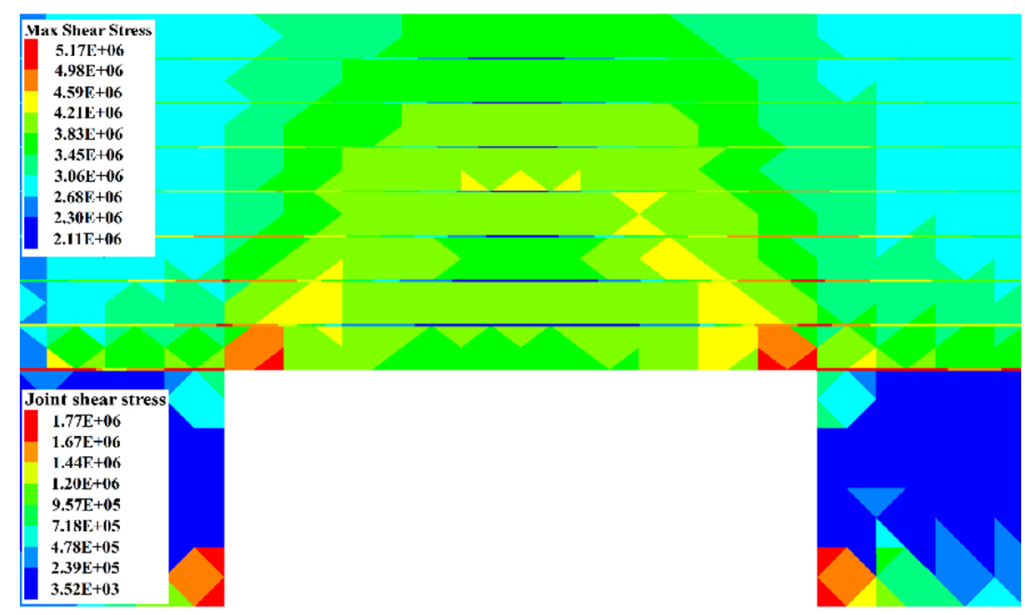

Fig. 8.31. Plot of maximum shear stress and joint shear stress magnitude at equilibrium for the model with bedded roof (unit: $\mathrm{Pa}$ )

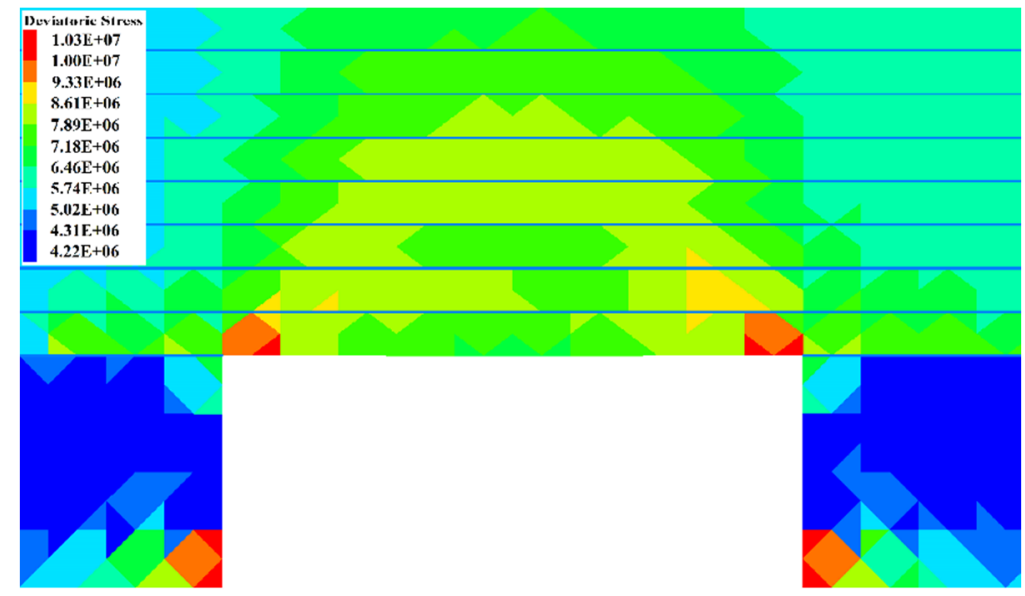

Fig. 8.32. Plot of deviatoric stress at equilibrium for the model with bedded roof (unit: Pa)

In addition, Fig. 8.30 plots the joint shear stress magnitude. Due to the difference in the vertical deformation of the rock layers, there is relative shear displacement between these layers, resulting in joint 
shear stress. From the distribution, it is notable that the minimum values occur at the center of the roof and that the joint shear stress gradually increases towards the hinge areas (the areas above the upper entry corners), where the maximum values occur. Fig. 8.30 marks the areas with large shear stress within the joints. The marked area on the right side is larger than left side, which is likely the result of directional horizontal stress. The existence of joint shear stress might affect the stress of the surrounding rock layers. However, Fig. 8.31 shows no apparent influence of the joint shear stress on the maximum shear stress. When comparing the failure profile shown in Fig. 8.30 and the distribution of maximum shear stress shown in Fig. 8.31, it illustrates that the area with high maximum shear stress surrounds the plastic zones. The deviatoric stress shows similar distribution in Fig. 8.32 as the maximum shear stress. The distribution of deviatoric stress shows the time-dependent strength degradation within the roof and the distribution of maximum shear stress can demonstrate the vulnerability to failure. The similarity in their distribution indicates that the time-dependent failure will propagate with the pattern determined by these stress distributions.

Furthermore, the following figures present the failure condition, major principal stress, and maximum shear stress. From the distribution of maximum shear stress and deviatoric stress in Figs. 8.31 and 8.32, it is likely that the failure would propagate to the surrounding areas. As shown in Fig. 8.33, the failure propagates within the bedding planes horizontally and also propagates upwards to the upper rock layer vertically from equilibrium condition to one day. Along with the failure propagation, the major principal stress and maximum shear stress further transfers to the surrounding areas, as shown in Figs. 8.34 and 8.35. Although the major principal stress on the right side is higher than the left side, there is no observed difference in the distribution of maximum shear stress. When comparing the stress magnitude at equilibrium (Figs. 8.29 and 8.31) and after one day (Figs. 8.34 and 8.35), it shows that the stress gradually redistributes with time and with the failure of roof rocks; while the overall stress magnitude gradually relaxes from the roof.

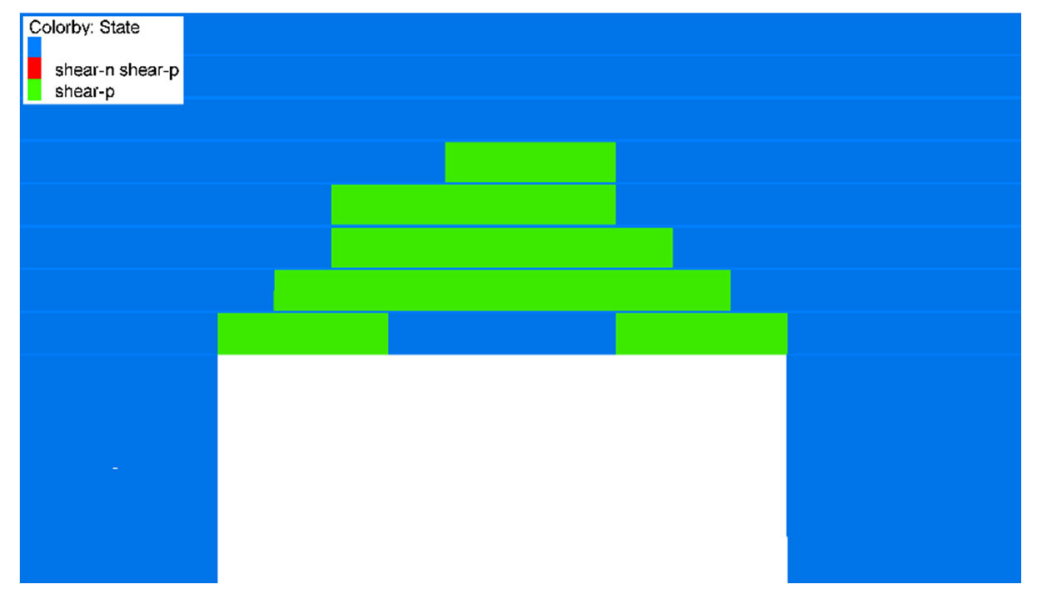

Fig. 8.33. Plot of failure condition after one day for the model with bedded roof 


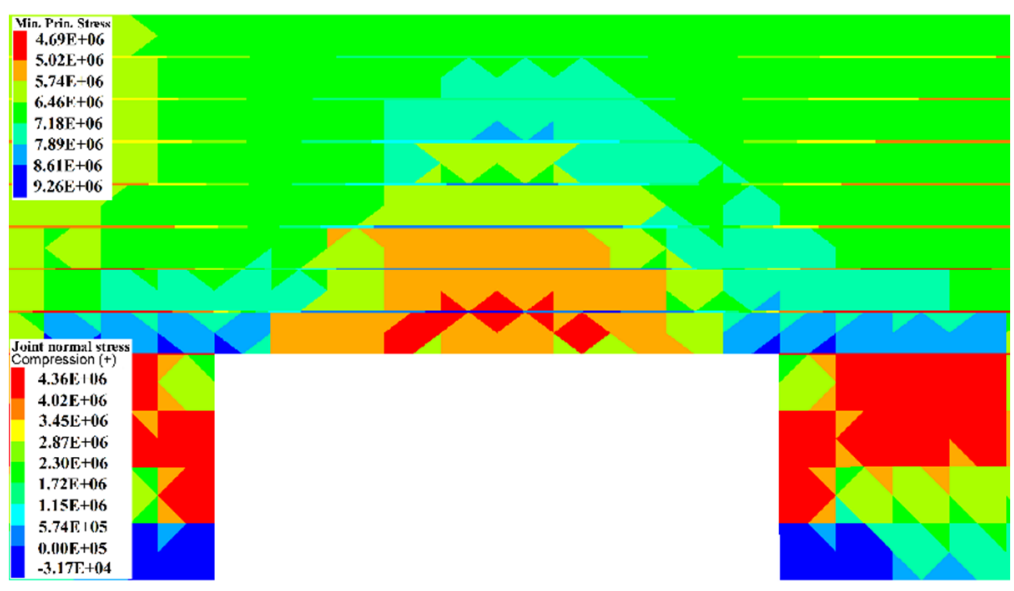

Fig. 8.34. Plot of major principal stress and joint normal stress after one day for the model with bedded roof

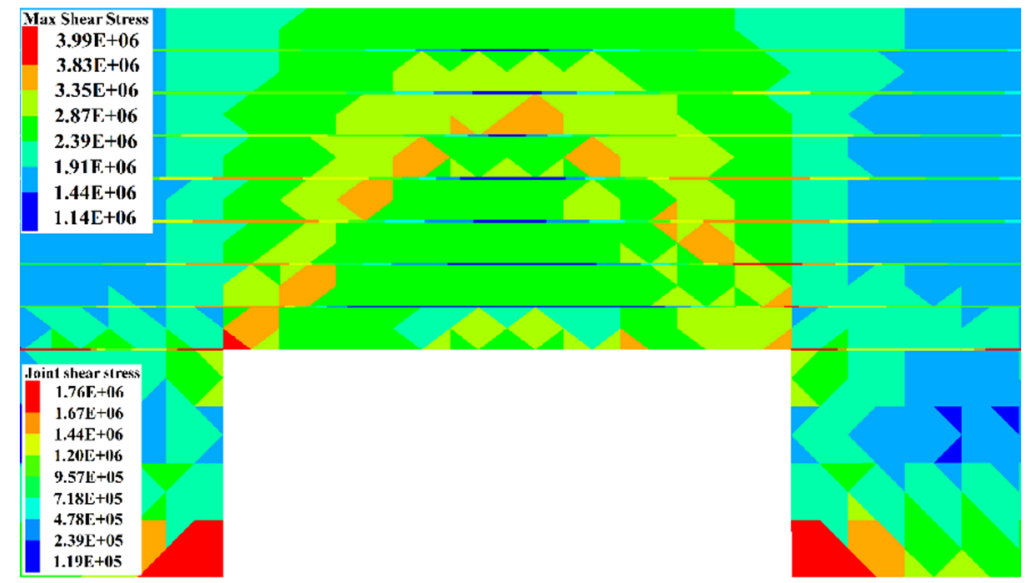

Fig. 8.35. Plot of maximum shear stress and joint shear stress after one day for the model with bedded roof

Finally, Fig. 8.36 plot the displacement magnitude and joint normal displacement magnitude. The presence of bedding planes divides the roof rock into layers. The resistance of the roof rock to bending decreases significantly. The first layer bends first, induced by the vertical load from the upper strata and its gravity. Once it bends, the support to the upper strata reduces, further increasing the deformation of the upper strata. As a result, the roof rock can deform into the entry, layer by layer. In addition, the analysis of the simplified beam model shows that increasing horizontal stress cannot help to build the compression arch to resist the bending within the beams if the beams are thin enough. Fig. 8.36 shows that the bedding plane are opening or separating symmetrically. The roof rocks also show symmetric displacement. The simulation results demonstrate that, under the simulated condition, the influence of directional horizontal stress on the roof deformation is insignificant and roof deformation is mainly affected by the presence of bedding planes. 


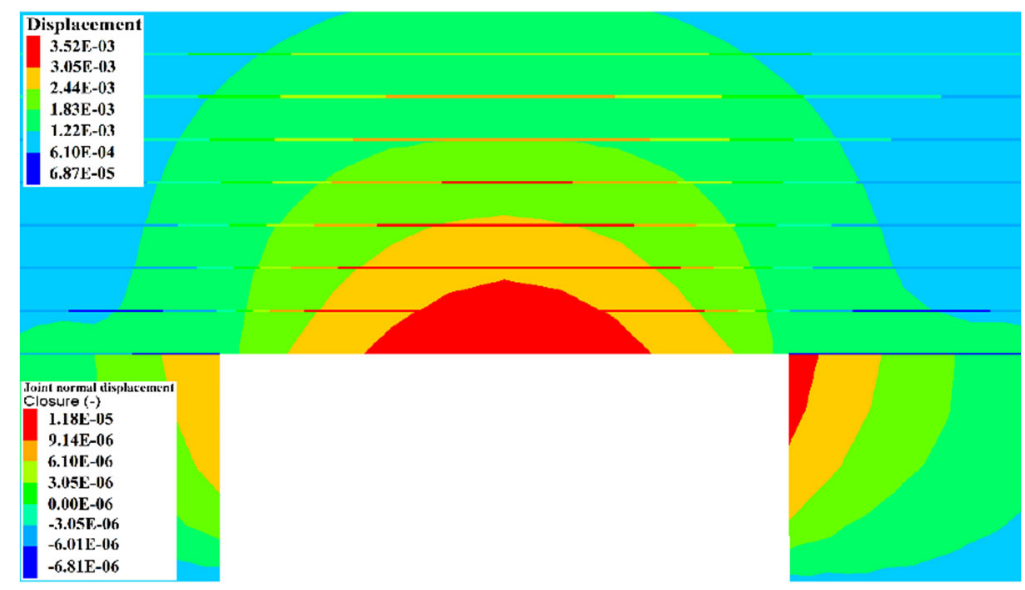

Fig. 8.36. Plot of displacement magnitude and joint normal displacement magnitude after one hour for the model with bedded roof

\subsubsection{Influence of bedding plane strength and stiffness on the time-dependent behavior of bedded roof}

The analysis of the simplified beam model shows that the bedding plane properties affect the shear stress within the bedding planes. As the joint shear stress can further affect the stress distribution within the rocks, the simulations used a low bedding plane strength and stiffness to investigate their influence on the timedependent behavior of bedded roof. From the failure condition at equilibrium, it is observable that as the strength and stiffness of the bedding planes reduces, there is a pronounced increase in the number of failed elements within the roof.

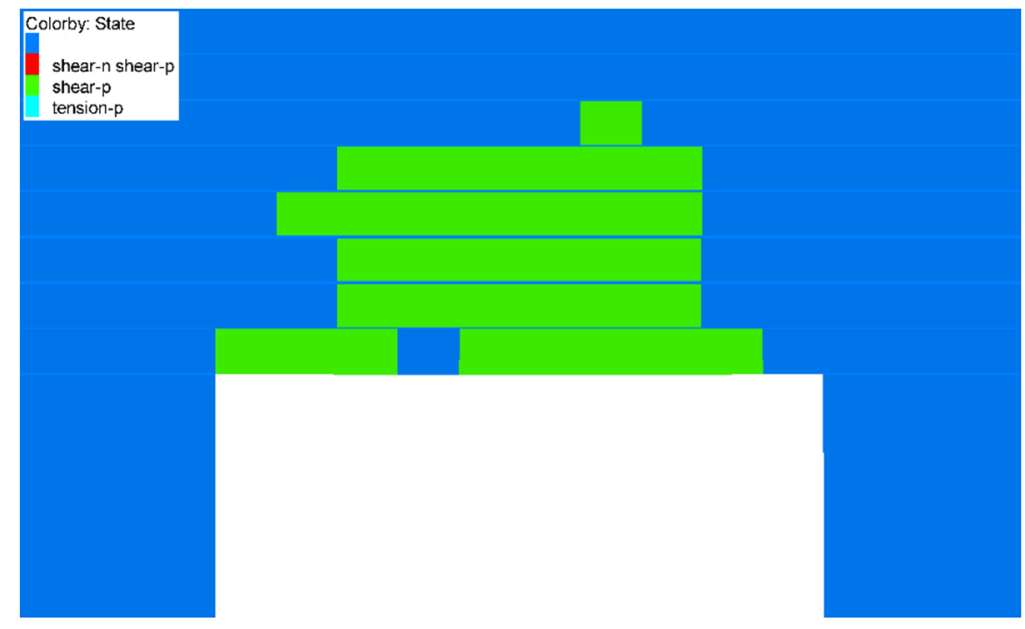

Fig. 8.37. Plot of failure condition at equilibrium for the model with bedding planes of low strength and stiffness

In addition, the analysis of the simplified beam model shows that the shear stress within the bedding planes varies with the bedding plane strength. With decreasing strength of the bedding planes, the peak joint shear stress increases, and its location moves towards the center of the roof. However, this behavior is not clear under the three-dimensional condition shown in Fig. 8.38. The introduction of failure into the threedimensional model completely changes the stress distribution. One common feature is the increase of the joint shear stress in the hinge area when the central part of the rock layers bend. Aside from this, Fig. 8.38 
shows the asymmetrical displacement distribution within the mine roof, different from the symmetric distribution of displacement in Fig. 8.36. The maximum displacement occurs at an area close to the right side of the entry, rather than at the center. In addition, the presence of bedding planes breaks the continuity of the displacement contour. The low strength and stiffness of the bedding plane decrease the connections between these rock layers and as a result, the roof can deform, layer by layer.

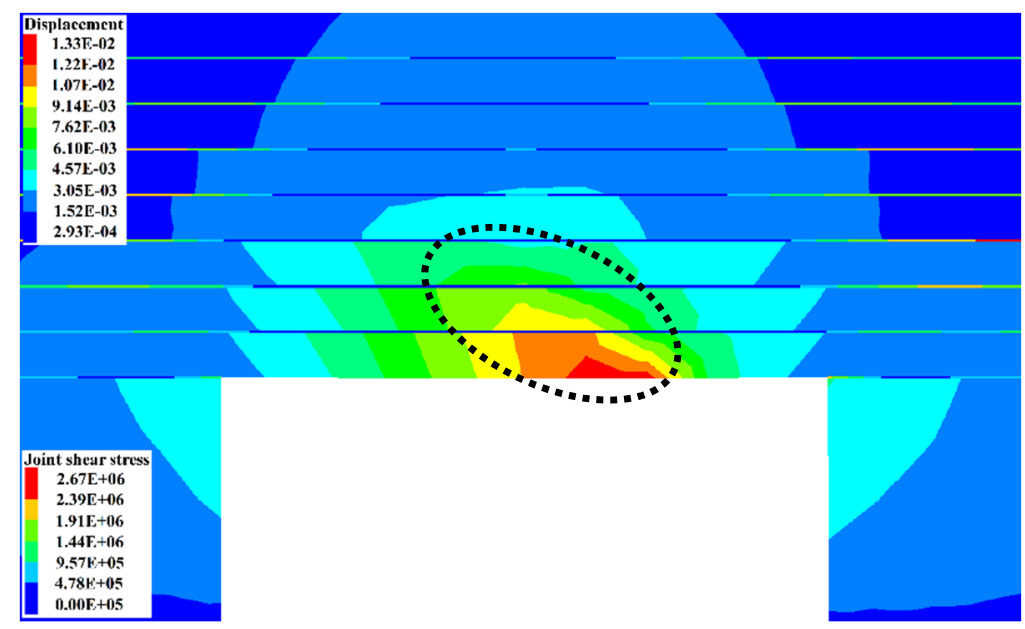

Fig. 8.38. Plot of displacement and joint shear stress magnitude at equilibrium for the model with bedding planes of low strength and stiffness

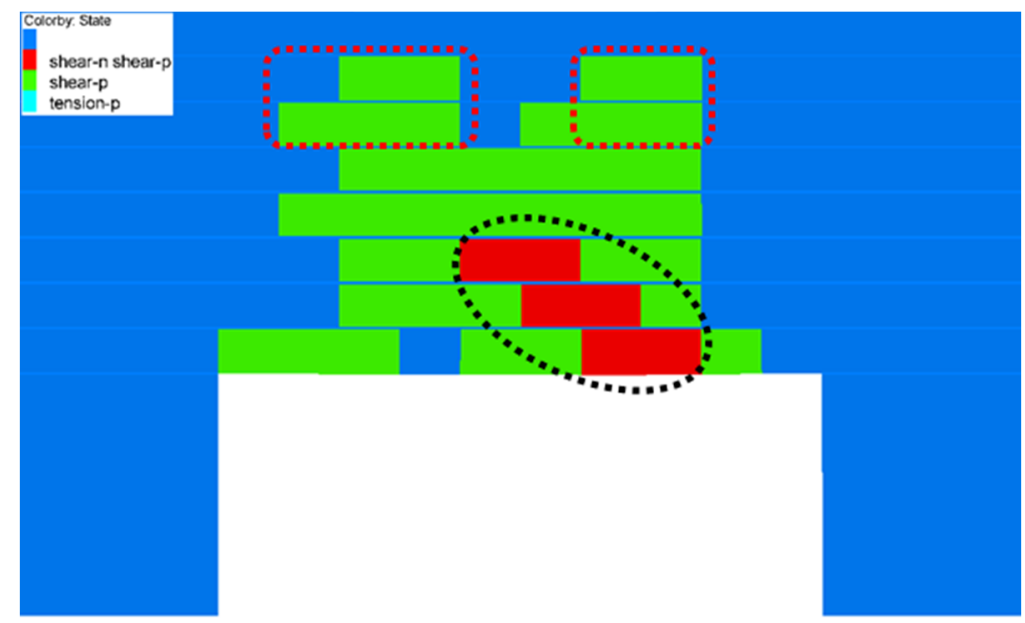

Fig. 8.39. Plot of failure condition after one day for the model with bedding planes of low strength and stiffness

Furthermore, this study investigated the failure condition and displacement magnitude of the bedded roof after one day, as shown in Figs. 8.39 and 8.40, respectively. From the failure condition, it is observable that the failure propagates upwards with time. The failure propagation starts from the right and left side of the failure zone and then changes to the horizontal direction. Another observation is that there are elements experiencing shear failure after one day within the failure zone. Within this zone, the stress is rapidly relaxing, and the occurrence of shear failure indicates that there is also stress redistribution occurring. When comparing Figs. 8.38 and 8.39, it is notable that the area experiencing shear failure after one day corresponds to the area where displacement concentrates at equilibrium. Fig. 8.40 illustrates that displacement within the mine roof still concentrates at this area, and the displacement further develops with 
time. Therefore, the concentrated displacement within this area changes the stress distribution and induces the shear failure.

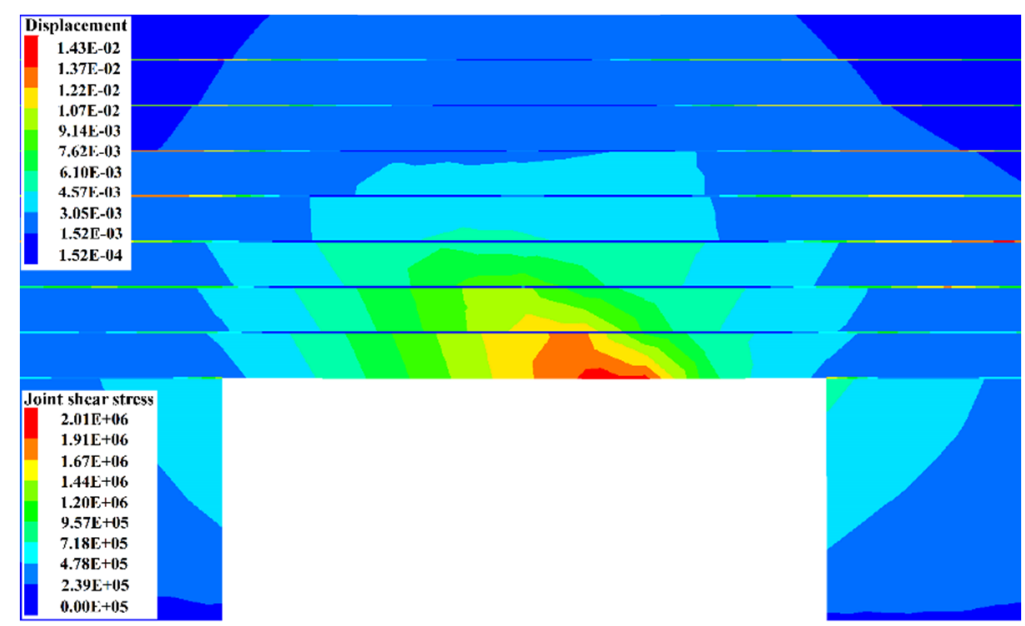

Fig. 8.40. Plot of displacement and joint shear stress magnitude after one day for the model with bedding planes of low strength and stiffness

Finally, Fig. 8.41 shows the contour of displacement magnitude and joint shear stress after one week. The time-dependent stress distribution indicates that the maximum shear stress and deviatoric stress are high at the locations surrounding the failed elements. They redistribute with the failure propagation, but always surround the failed elements. With the movement of the elements with high maximum shear stress and deviatoric stress, failure propagates, and the time-dependent displacement gradually accelerates. The comparison between Figs. 8.40 and 8.41 shows that the displacement within the mine roof gradually develops with time. As marked in Fig.8. 41, the joint shear stress also gradually develops inside the roof, along with the displacement developing deeply into the roof.

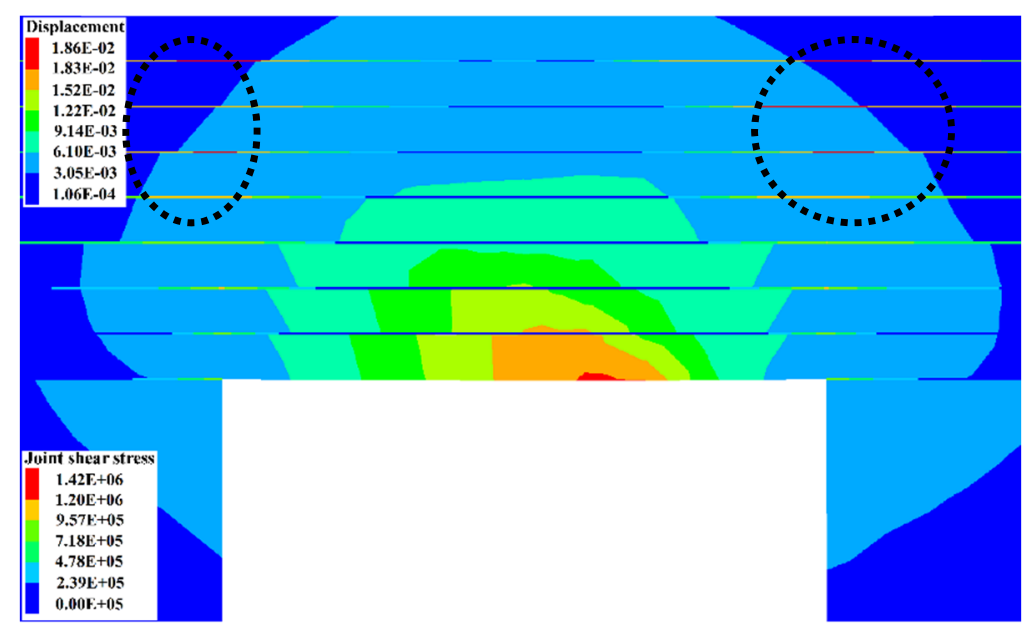

Fig. 8.41. Plot of displacement and joint shear stress magnitude after one week for the model with bedding planes of low strength and stiffness

\subsubsection{Step-wise excavation of bedded mine roof}

The simulation of massive roof with step-wise excavation shows that the failure process and 
displacement development can be different due to the stress path resulting from the step-wise excavation. Therefore, it is necessary to study the behavior of bedded mine roof with step-wise excavation. The simulation is similar to the step-wise simulation with massive roof. At each mining step, the simulation excavated one section of entry with a length of $0.3 \mathrm{~m}(1 \mathrm{ft})$. The model ran to equilibrium first, and the time-dependent simulation ran for the corresponding time of excavation. Afterwards, it simulated the next

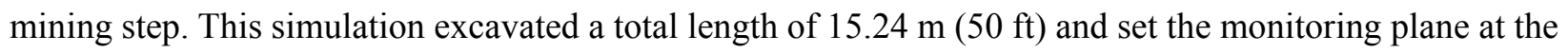
location of $7.62 \mathrm{~m}(25 \mathrm{ft}$ ) from the front surface of the model. Figs. 8.42 through Fig. 8.45 present the failure condition and joint shear stress at the monitoring location after the $26^{\text {th }}, 30^{\text {th }}, 35^{\text {th }}$, and $50^{\text {th }}$ mining step. They cover the situations of the mining face just passing the monitoring location and gradually moving far away from the monitoring location.

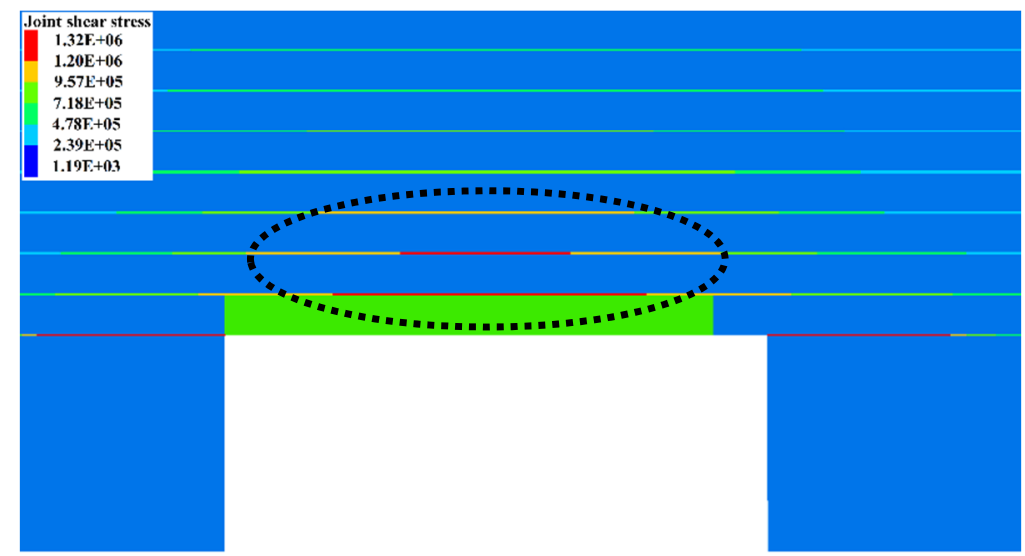

Fig. 8.42. Plot of failure condition and joint shear stress at 26th mining step

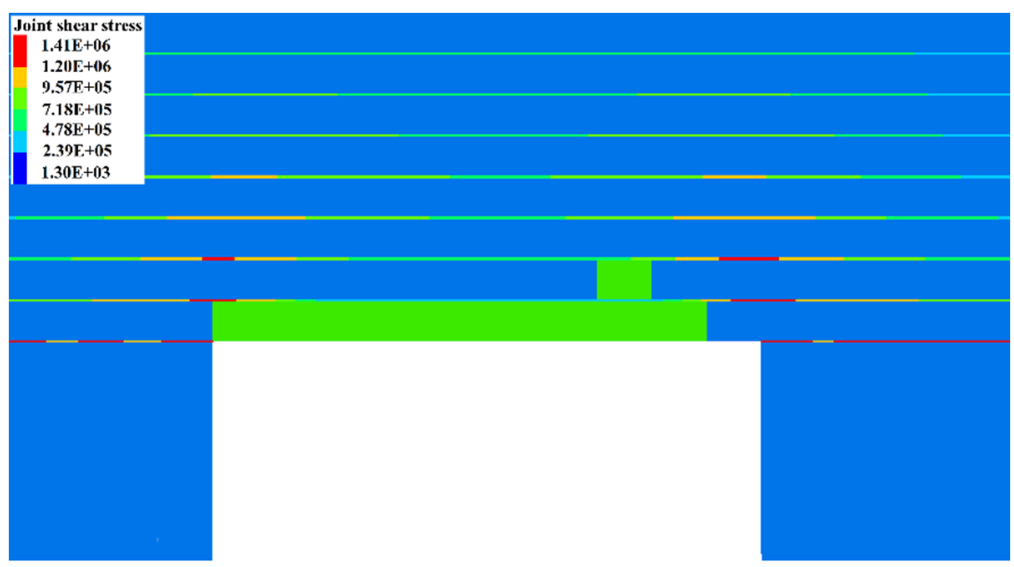

Fig. 8.43. Plot of failure condition and joint shear stress at 30th mining step 


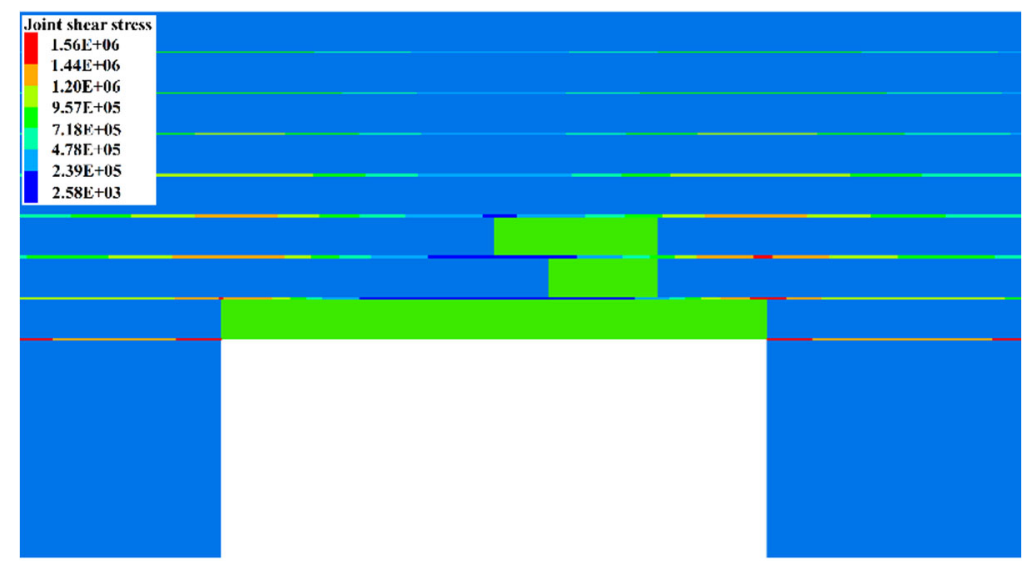

Fig. 8.44. Plot of failure condition and joint shear stress at 35 th mining step

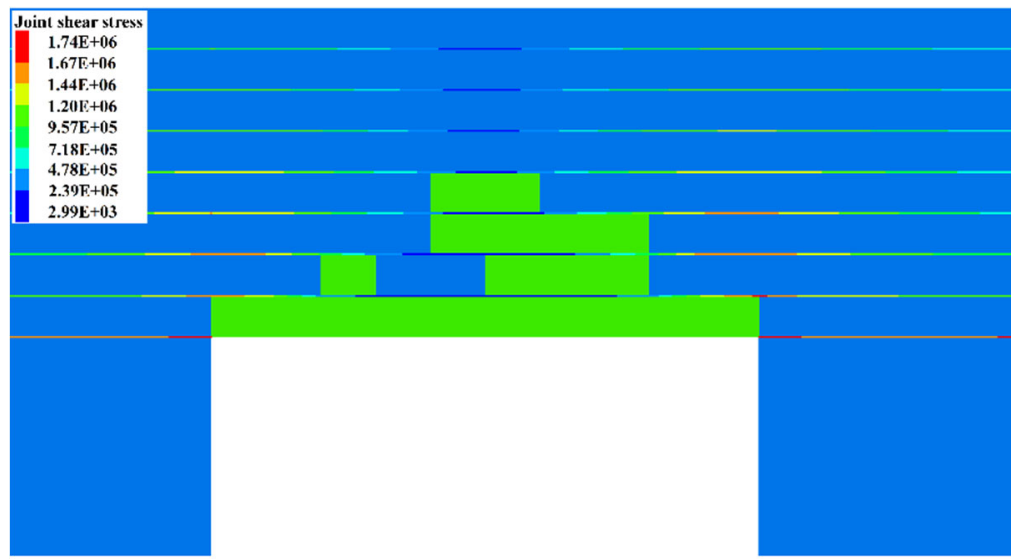

Fig. 8.45. Plot of failure condition and joint shear stress at 50th mining step

The failure condition after $50^{\text {th }}$ mining step is different from that with the one-step excavation shown in Fig. 8.28. Although the failure occurs mostly at the central part of the roof for both situations, there is less failure occurring in the model with step-wise excavation. The major difference is the failure within the first layer of the roof. For the model excavated in one step, there is only failure occurring close to the corners. However, the whole layer fails for the model with step-wise excavation. The failure process in Figs. 8.42 through Fig. 8.45 shows that when the mining face just passed the monitoring location, the first layer has already failed, and the upper layers also gradually fails with the movement of the mining face. This indicates that the gradual removal of coals affects the behavior of the first roof layer. With one-step excavation, the first roof layer (a plate) lose the constraint or confinement from the coal at the same time and start to deform downwards. However, with the step-wise simulation, the constraint or confinement to the roof is gradually released. The stress within the roof experiences an increase first and then a reduction along with the stress transfer into deeper area, as shown in Fig. 7.30. Therefore, the stress path resulted from the step-wise excavation leads to the different failure profiles.

In addition, the step-wise excavation affects the shear stress within the bedding planes. Fig. 8.42 illustrates that high joint shear stress occurs at the central part of the roof. Normally, studies have considered that there is very low or no shear stress at this location due to the roof sag. However, the step-wise simulation shows that this is not true in all cases. Fig. 8.42 through Fig. 8.45 show that when the mining face is close 
to the monitoring location, there is very large shear stress within the bedding planes at the central part of the roof, and the joint shear stress gradually decreases when the mining face advances. When the face passes the monitoring location and is close, the confinement from the coal is partially removed and the coal at the mining face can still constrain the deformation at the monitoring location. This condition induces shear stress along the entry direction, not the cross-section direction. When the advancement of the face, the constraint along the entry direction is gradually removed and deformation of the roof concentrates in the vertical direction. The bending of the beam further promotes the build-up of joint shear stress above entry corners, as shown in Fig. 8.43 through Fig. 8.45. Therefore, the bedding planes within immediate roof experience high shear stress during entry development. The high joint shear stress potentially induces shear failure along the bedding planes if the joint strength is low. Once the bedding plane fails by shear, large roof sag can occur when the face moves far away.

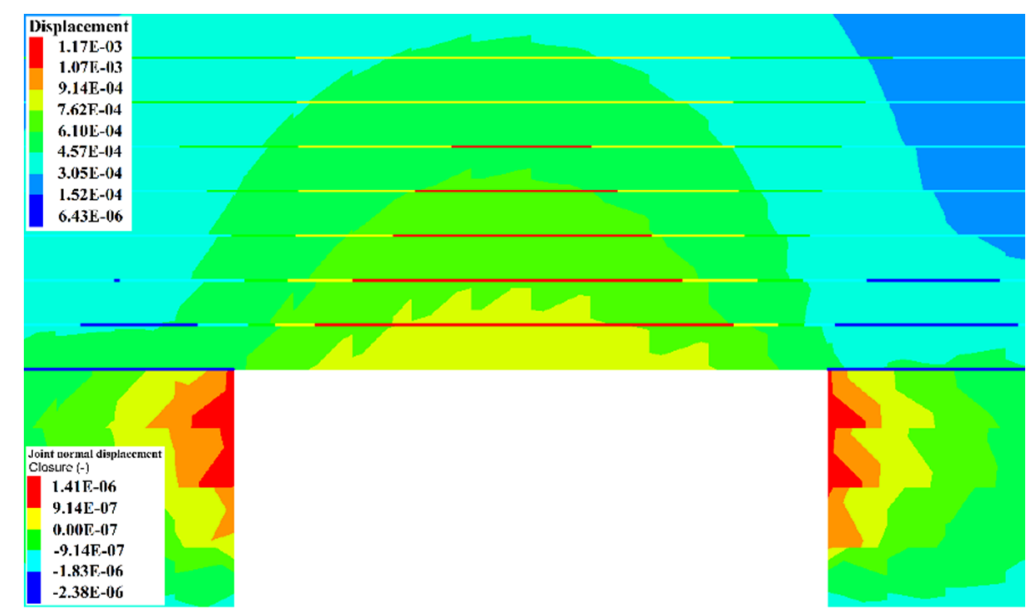

Fig. 8.46. Plot of displacement magnitude and joint normal displacement magnitude at 26th mining step

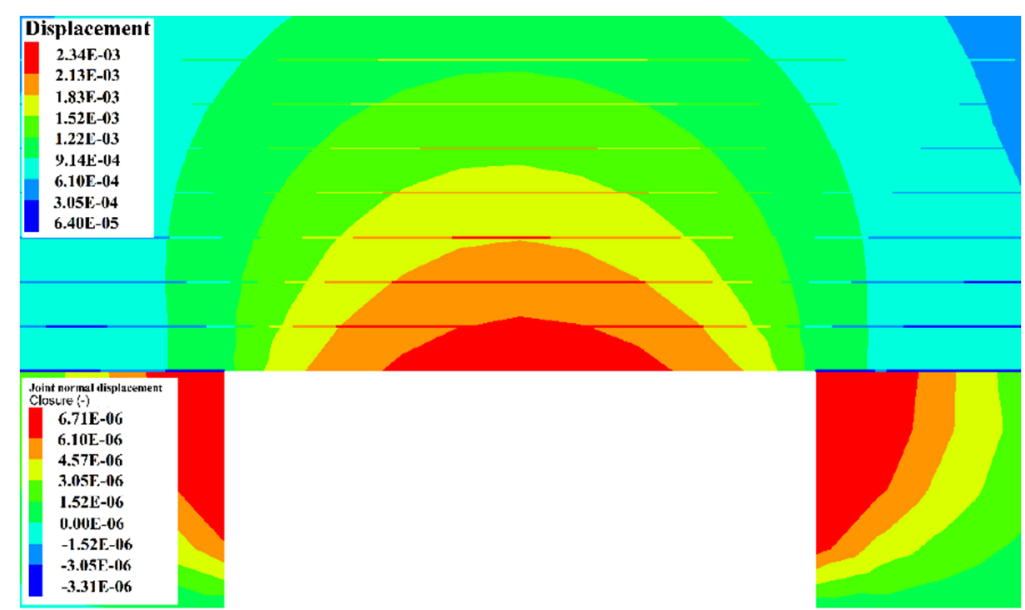

Fig. 8.47. Plot of displacement magnitude and joint normal displacement magnitude at 50th mining step

Finally, this study investigated the displacement distribution during the entry development. The stepwise excavation with massive roof shows asymmetrical displacement distribution with the entry development. However, there is no asymmetrical displacement observable in the step-wise simulation of bedded roof. Due to the bending of the rock layers, the maximum displacement occurs at the center of the roof and gradually decreases towards the pillars. 


\subsection{SUMMARY}

The shale rock in the immediate roof of underground coalmines is normally bedded or laminated. The introduction of bedding planes in the immediate roof decreases the rock strength and introduces anisotropy affecting the roof failure mode. The presence of bedding planes can also affect the time-dependent response of the mine roof. The purpose of this chapter is to investigate the time-dependent behavior of bedded mine roof.

When the mine roof consists of bedded strata, studies usually analyze the rock layers as rock beams. This chapter proposed a simplified beam model to investigate the influence of bedding planes on the stress distribution of mine roof. Under in-situ conditions, the horizontal and vertical stress applied onto the beams are balanced. After mining, the vertical stress from the coal seam releases. The vertical stress from the upper strata and the gravity of the beam itself generates bending torque onto the beam. When the thickness of the beam is large enough, a compression arch generates inside the beam to resist the bending. Due to the high stress, shear failure can initiate along this compression arch. However, a thin beam cannot generate such a compression arch, and thus has very low resistance to bending torque. The bending of the rock layers leads to roof sag and the increase in the shear stress at the hinge section, which is the area above the upper entry corners. The increasing shear stress potentially induces shear failure at these locations. The time-dependent behaviors of the roof rock make the rock beam softer and softer, further reducing the resistance of the rock beam to bending. Due to the stress relaxation, the stress within the roof gradually decreases with time. However, the bending of the rock layers in turn increases the shear stress at the hinge sections and potentially induces time-dependent failure from these areas. In addition, the influence of the bedding planes also depends on the properties of the bedding planes. The joint shear stress can be different when bedding planes have different strengths.

Furthermore, this study simulated the time-dependent behavior of bedded mine roof by using the developed viscoelastic-strain-softening model under three-dimensional conditions. Although the influence of directional horizontal stress on the major principal stress distribution is observable, its influence on the failure profile, maximum shear stress, and deviatoric stress is insignificant. The inclusion of bedding plane into the mine roof increases the freedom of deformation for the bedded roof; the roof can deform from the roofline, layer by layer. Due to the bending and deformation of the rock layers, the principal stress highly reduces at the central part of the roof. Failure concentrates at this location, obtaining more failure than in the massive roof. Along with the bending of the rock layers, large joint shear stress occurs at the hinge sections of each layer. As the maximum shear stress and deviatoric stress are observed at the areas surrounding the failure zone at equilibrium, the failure gradually propagates towards the surrounding areas horizontally and vertically. In addition, this study investigated the influence of bedding plane strength and stiffness by reducing the values of these factors in the simulation; the results show that more failure induces in the roof. Along with the failure propagation and beam deformation, the joint shear stress gradually develops deeply into the roof. Concentrated displacement occurred at one location close to the right side of the entry within the roof, which further affected the stress distribution and induced shear failure. Finally, adopting the step-wise simulation method facilitated the study of the time-dependent behavior of bedded roof. Due to the progressive removal of the coal, a different stress path was achieved for the step-wise 
simulation from the one-step excavation and, as a result, the simulation obtained a different failure profile. The results demonstrate that the simulation method also affect the joint stress. When the mining face is close, there is high shear stress within the bedding planes at the central part of the roof. Along with the advance of the face, the joint shear stress at the center reduces significantly. At the same time, due to the removal of the constraint from the coal, the bending of the rock layers increases leading to the increase in joint shear stress at the hinge areas of each layer. If the joint strength is low, the high joint shear stress when the mining face is close may induce safety problem. 


\section{CHAPTER 9 CONCLUSIONS AND RECOMMENDED FUTURE STUDIES}

\subsection{CONCLUSIONS}

As a leading source of injury in underground coal mines, studies have investigated roof falls for decades, drawing several important conclusions. However, there are limited studies on the time-dependent behavior of roof rock. Field observations demonstrate that the formation of roof falls is a time-dependent process. After excavation, the roof continues to converge over time, and the roof convergence curve shows the timedependent behavior of the mine roof. The accelerating convergence at the end of a classic roof convergence curve is a sign of impending roof instability. However, the time-dependent behavior of roof is much more complex than the measured data; this is due to the fact that the time-dependent failure of mine roof involving various rock mechanics processes, including the time-dependent behavior of both intact and failed coal measure rocks, rock strength degradation with time, and the time-dependent failure process. Aiming to solve these problems, this study proposed and verified the relaxation test as an alternative test method to study the time-dependent behavior and property of rocks; it also proposed and programmed a constitutive model capable of representing all the observed time-independent and time-dependent rock behaviors into 3DEC as a user-defined model to simulate the time-dependent failure process of massive and bedded mine roof. This dissertation draws the following conclusions from the studies performed throughout this process:

$>$ The rocks within the mine roof do not experience purely creep (constant load) or purely relaxation (fixed displacement), rather the rock response is a combination of both creep and relaxation.

$>$ Compared with creep tests, the relaxation test is the better method to study the time-dependent behavior and property of rocks. Creep tests can only be conducted in the pre-failure region, while relaxation test can be conducted at any stage of a compression test. Therefore, the relaxation test can determine the time-dependent property of rocks at any state, pre- or post-failure.

$>$ Intact rock shows a typical smooth stress relaxation behavior, while failed rock shows a step-wise relaxation behavior. During a relaxation test on failed specimens, the rock experienced sudden and large stress drops after a stage of smooth relaxation. The sudden stress drops are likely a result of the propagation of the major failure plane or the shearing of asperities on the failure plane after a stage of time-dependent sliding along the major shear failure plane.

$>$ There is a significant variation in the time-dependent properties between intact and failed rocks. Even within the residual stage, when the initial stress is very low, the ratio of relaxed stress to the stress initiating the relaxation test is higher than that in the pre-failure region; however, the variation in the time-dependent properties of failed rocks is insignificant and ignorable. As a result, it is reasonable to use two groups of viscous parameters to represent the time-dependent properties of the intact and the failed rocks. 
The variation in the time-dependent properties can induce time-dependent failure propagation. Rapid stress relaxation in a failure zone decreases the confinement to the surrounding intact rock and increases the differential stress, potentially inducing failure at the boundary of the failure zone. However, this time-dependent failure process depends on various factors, like the size and location of the failure zone at equilibrium, the in-situ stress condition, and the difference in the timedependent properties between intact and failed rock. There is an observable increase in roof convergence as time-dependent failure occurs within the mine roof.

$>$ Rock strength degrades with accumulated viscous deformation, regardless of other environmental influences. The relaxation test confirmed this degradation. The intact specimen did not fail when the relaxation test initiated, and failure occurred when the stress was relaxing from the specimen. However, it is not easy to develop the relationship between strength and effective viscous strain solely from relaxation tests, rather the recommended option is to conduct creep tests to develop the strength degradation law.

$>$ The influence of strength degradation on the time-dependent failure propagation is highly dependent upon the stress distribution. The strength degradation is controlled by the accumulated, effective viscous strain, which further depends on the deviatoric stress. The situation becomes much more complex when considering the variation in the time-dependent properties. As the variation in the time-dependent properties can affect the stress distribution, the differential stress increases and the rock deterioration accelerates at the boundary of the failure zone.

Regardless of the mechanism(s) for the viscous behaviors of rocks, the accumulation of viscous deformation within rock decreases the rock's resistance to deviatoric stress. In other words, the viscous behaviors of rock reduce the stiffness of rocks and make them gradually softer. For bedded roof, reducing the rock stiffness will increase the roof sag.

For massive mine roof, failure initiates from the entry upper corners and within an arch-shaped band, with high deviatoric stress existing within the immediate roof. The accumulation of viscous deformation deteriorates roof rocks within the band, and failure potentially propagates along the arch-shaped band. Once the rock fails, shear plastic strain accumulates, further decreasing the strength of the failed rock. After the failures initiating from the entry corners coalesce along with the arch-shaped band, the failure changes to propagate horizontally. At the same time, the stress relaxes from the roof and potentially prevents further failure propagation. The time-dependent stress distribution makes the time-dependent deformation rate inside the roof vary with location and time.

$>$ Directional horizontal stress has a significant influence on time-dependent roof stability. An increase in the maximum horizontal stress can increase the failure and stress magnitude at equilibrium, further promoting the failure with time.

When the thickness of the rock beam within mine roof is large enough, a compression arch generates inside the beam to resist bending. Due to the high stress, shear failure can initiate along the compression arch. In addition, the bending and stress within the beam can break the compression 
arch, further decreasing the resistance of the beam to bending.

A thin rock beam cannot generate a compressive arch and thus has very low resistance to bending. The bending of the rock layers leads to roof sag and an increase in shear stress at the hinge areas. The viscous behavior of the roof rock reduces the resistance of the rock beam to bending over time. The stress within the roof gradually relaxes over time; the bending of rock layers, conversely, increases the shear stress at the hinge areas. Therefore, it is necessary to install roof support as soon as possible to prevent and decrease roof sag.

Bedding planes increase the freedom of deformation of bedded roof. The roof can deform from the roofline, layer by layer. The bending and deformation of the rock layers greatly reduces the principal stress at the central part of the roof and, as a result, failure concentrates in this area. Along with the failure, stress relaxation, and deformation, the time-dependent deformation gradually moves deeper into the roof, further inducing more time-dependent failure.

$>$ A step-wise simulation can reproduce the stress path that the roof rock experiences during entry development to obtain more realistic simulation results. With step-wise excavation, the mine roof experiences an increase in stress when the face moves toward the monitoring location and a gradual release of confinement from the underlying coal seam after the face passes, obtaining a stress path different from that of one-step excavation. A step-wise simulation with massive roof shows the change in the displacement distribution along with the failure propagation within the mine roof. For a bedded roof, a step-wise simulation demonstrates that there is high shear stress within the bedding planes at the midspan of the roof when the face is close; if the joint strength is low, the high joint shear stress becomes a safety concern.

> The stress distribution within the mine roof is very critical for insuring long-term stability. Simulation results show that the strength deterioration relies on the distribution of deviatoric stress, as well as how the time-dependent failure propagation follows the distribution of major principal stress. Support methods that can reduce the unfavorable stress distribution within the mine roof are preferable.

\subsection{RECOMMENDED FUTURE STUDIES}

This study proposed and verified the relaxation test to determine the time-dependent property of rocks. First, unconfined relaxation tests were performed on various coal measure rocks. Next, a constitutive model capable of representing the observed time-independent and time-dependent behaviors was proposed and programmed into 3DEC to simulate the time-dependent behaviors of massive and bedded mine roof. However, there are several studies that need to occur in the future to continue this investigation.

First, researchers should use triaxial relaxation tests to study the relaxation behavior of intact and failed rocks under triaxial conditions. This study only conducted unconfined relaxation tests; it is likely the presence of confining pressure will inhibit the variation in the time-dependent property.

In addition, a viscoplastic model can represent the time-dependent behavior of rocks, especially in the 
post-failure region. In this study, Burgers model (a viscoelastic model) represented the time-dependent behavior of rocks. This indicates that once the stress reduces, the viscous strain will recover over time. In the post-failure region, the time-dependent deformation cannot recover, especially the time-dependent sliding along the major failure plane. Therefore, a viscoplastic model may be a better option to represent the time-dependent behavior in the post-failure region. In addition, studies could also incorporate timedependent dilation behavior into the viscous model. 


\section{References}

Aggson JR (1979) Design of room and pillar mining systems. In: First Conference on Ground Control Problems in the Illinois Coal Basin. Carbondale, Illinois

Aggson JR, Mouyard DP (1988) Geomechanical evaluation of a coal mine arched entry. Geotech Geol Eng 6:185-193

Amadei B (1979) Creep behaviour of rock joints. University of Toronto

Ashby M, Sammis C (1990) The damage mechanics of brittle solids in compression. Pure Appl Geophys 133:489-521

Azzouz AS, Germaine JT (1981) Behavior of cylindrical openings in strain-softening ground. In: 22nd US Symposium on Rock Mechanics. Cambridge, Massachusetts, USA, pp 258-263

Barla G (2009) Innovative Tunneling Construction Method to Cope with Squeezing at the Saint Martin La Porte Access Adit (Lyon-Turin Base Tunnel). In: ISRM Regional Symposium - EUROCK 2009. pp 15-24

Barla G, Debernardi D, Sterpi D (2012) Time-Dependent Modeling of Tunnels in Squeezing Conditions. Int J Geomech 12:697-710. doi: 10.1061/(ASCE)GM.1943-5622.0000163

Barron K, Baydusa A (1999) A theory of cutter roof failure and its application. CIM Bull 92:45-49

Baud P, Meredith PG (1997) Damage accumulation during triaxial creep of darley dale sandstone from pore volumometry and acoustic emission. Int J Rock Mech Min Sci 34:24.e1-24.e10

Bieniawski ZT (1970) Time-dependent behaviour of fractured rock. Rock Mech 2:123-137

Bowden RK, Curran JH (1984) Time-dependent behavior of joints in shale. In: The 25th symposium on rock mechanics-rock mechanics in productivity and protection. pp 320-327

Brantut N, Baud P, Heap MJ, Meredith PG (2012) Micromechanics of brittle creep in rocks. J Geophys Res 117:B08412

Challamel N, Lanos C, Casandjian C (2005) Creep damage modelling for quasi-brittle materials. Eur J Mech A/Solids 24:593-613

Chenevert ME, Gatlin C (1965) Mechanical Anisotropies of Laminated Sedimentary Rocks. Soc Pet Eng J 5:67-77

Chin H, Rogers JD (1987) Creep parameters of rocks on an engineering scale. Rock Mech 20:137-146

Chungh Y, Missavage RA (1981) Effects of moisture on strata control in coal mines. Eng Geol 17:241255

Coggan J, Gao F, Stead D, Elmo D (2012) Numerical modelling of the effects of weak immediate roof lithology on coal mine roadway stability. Int J Coal Geol 90-91:100-109

Costin LS, Mecholsky JJ (1983) Time dependent crack growth and failure in brittle rock. In: 24th US Symposium on Rock Mechanics. pp 385-394

Cruden DM (1971) Single-increment creep experiments on rock under uniaxial compression. Int J Rock Mech Min Sci 8:127-142 
Cruden DM (1974) The static fatigue of brittle rock under uniaxial compression. Int J Rock Mech Min Sci Geomech Abstr 11:67-73

Debernardi D, Barla G (2009) New viscoplastic model for design analysis of tunnels in squeezing conditions. Rock Mech Rock Eng 42:259-288

Dolinar DR, Mucho TP, Oyler DC, Pablic J (2000a) Utilizing the “Advance and Relieve” Method To Reduce Horizontal Stress Affects on the Mine Roof, a Case Study. In: 19th Conference on Ground Control in Mining

Dolinar DR, Mucho TP, Oyler DC, Pabtic J (2000b) Utilizing the "Advance-and-Relieve" Method to reduce horizontal stress effects on the mine roof, a case study. In: 19th Conference on Ground Control in Mining. Morgantown, WV

Dragon A, Mroz Z (1979) A model for plastic creep of rock-like materials accounting for the kinetics of fracture. Int J Rock Mech Min Sci Geomech Abstr 16:253-259

Drescher K, Hardley MF (2003) Aspects of time-dependent deformation in hard rock at great depth. J South African Inst Min Metall 103:325-335

Einstein HH, Hirschfeld RC (1973) Model studies on mechanics of jointed rock. J soil Mech Found Div 99:229-248

Esterhuizen G, Bajpayee T (2012) Horizontal stress related failure in bedded mine roofs - insight from field observations and numerical models. In: The 46th US Rock Mechanics/Geomechanics Symposium. Chicago, IL, USA

Esterhuizen GS, Dolinar DR, Iannacchione AT (2008) FIELD OBSERVATIONS AND NUMERICAL STUDIES OF HORIZONTAL STRESS EFFECTS ON ROOF STABILITY IN U . S . LIMESTONE MINES. J South African Inst Min Metall 108:345-352

Fakhimi A, Fairhurst C (1994) A model for the time-dependent behavior of rock. Int J Rock Mech Min Sci Geomech Abstr 31:117-126

Fang Z, Harrison JP (2001) A mechanical degradation index for rock. Int J Rock Mech Min Sci 38:11931199

Fang Z, Harrison JP (2002) Development of a local degradation approach to the modelling of brittle fracture in heterogeneous rocks. Int J Rock Mech Min Sci 39:443-457

Gadde M, Peng S (2004) Effect of in-situ stresses on the stability of coal mine development workings. Proc 23rd Int Conf Gr Control Min 92-102

Gadde M, Rusnak J, Honse J, Peng SS (2007) On rock failure criteria for coal measure rocks. In: 26th International Conference on Ground Control in Mining, Morgantown, USA. Morgantown, WV, pp 361-369

Gadde MM, Peng SS (2005) Numerical simulation of cutter roof failure under weak roof conditions. In: SME Annual Meeting. Salt Lake City, UT, USA

Gale WJ, Nemcik JA, Upfold RW (1987) Application of stress control methods to underground coal mine design in high lateral stressfields. In: 6th ISRM Congress. Montreal, Canada, pp 897-900

Gao F, Stead D (2013) Discrete element modelling of cutter roof failure in coal mine roadways. Int J Coal Geol 116-117:158-171

Gao F, Stead D, Kang H (2014) Simulation of roof shear failure in coal mine roadways using an 
innovative UDEC Trigon approach. Comput Geotech 61:33-41

Ghorbani M, Sharifzadeh M (2009) Long term stability assessment of Siah Bisheh powerhouse cavern based on displacement back analysis method. Tunn Undergr Sp Technol 24:574-583

Gioda G (1981) A finite element solution of non-linear creep problems in rocks. Int J Rock Mech Min Sci Geomech Abstr 18:35-46

Gioda G, Cividini A (1981) Viscous behaviour around an underground opening in a two-phase medium. Int J Rock Mech Min Sci Geomech Abstr 18:437-443.

Goodman RE (1989) Introduction to rock mechanics-second edition

Hagros A, Johansson E, Hudson JA (2008) Time dependency in the mechanical properties of crystalline rocks. A literature survey

Hajiabdolmajid V, Kaiser PK, Martin CD (2002) Modelling brittle failure of rock. Int J Rock Mech Min Sci 39:731-741

Hamiel Y, Liu Y, Lyakhovsky V, et al (2004) A viscoelastic damage model with applications to stable and unstable fracturing. Geophys J Int 159:1155-1165

Heap M (2009) Creep: Time-dependent brittle deformation in rocks. University College London

Hobbs DW (1970) Strss-strain-time behavior of a number of coal measure rocks. Int J Rock Mech Min Sci Geomech Abstr 7:149-170

Hudson A, Brown T (1972) Studying time-dependent effects in failued rock. In: The 14th U.S. Symposium on Rock Mechanics (USRMS). University Park, Pennsylvania, pp 25-34

Hyams D (2010) CurveExpert Basic 1.4

Iannacchione A, Coyle P, Prosser L, et al (2004) The relationship of roof movement and strata-induced microseismic emissions to roof falls. In: 2004 SME Annual Meeting. Denver, pp 1-9

Itasca (2012) 3DEC-Three-dimensional distinct element code version 5.0 user's manual

Ito H (1983) Creep of rock based on long-term experiments. In: 5th ISRM Congress. Melbourne, Australia, pp 117-120

Kaiser PK (1980) Effect of stress history on the deformation behavior of undergroun openings. In: 13th Canadian Rock Mechanics Symposium. pp 133-140

Kaiser PK (1981) Phenomenological model for rock with time-dependent strength. Int J Rock Mech Min Sci Geomech Abstr 18:153-165

Kemeny J (1991) A model for non-linear rock deformation under compression due to sub-critical crack growth. Int J Rock Mech Min Sci Geomech Abstr 28:459-467

Kemeny J, Cook N (1987) Determination of rock fracture parameters from crack models for failure in compression. 28th US Symp Rock Mech 367-374

Kimura T, Esaki T, Kameda N, Nishida T (1987) Experimental and theoretical studies on strain softening behavior of rocks. In: 28th US Symposium on Rock Mechanics. Tucson, AZ, USA

Kranz RL, Scholz CH (1977) Critical Dilatant Volume of Rocks at the Onset of Tertiary Creep. J Geophys Res 82:4892-4895 
Kulatilake PHSW (1985) Estimating Elastic Constants and Strength of Discontinuous Rock. J Geotech Eng 111:847-864

Lau JSO, Chandler N a. (2004) Innovative laboratory testing. Int J Rock Mech Min Sci 41:1427-1445

Li Y, Ghassemi A (2012) Creep Behavior of Barnett, Haynesville, And Marcellus Shale. 46th U.S. Rock Mech. Symp. 7

Lin QX, L.G.Tham, M.R.Yeung, Lee PKK (2004) Failure of Granite under constant loading. Int J rock Mech Min Sci 41:362

Lin QX, Liu YM, Tham LG, et al (2009) Time-dependent strength degradation of granite. Int J Rock Mech Min Sci 46:1103-1114

Lockner D (1993) Room temperature creep in saturated granite. J Geophys Res 98:475-487

Lorig L, Calderon Rojo A (2002) Modeling time-dependent slope deformation. In: ISRM International Symposium-EUROCK 2002. Internatioanl Society for Rock Mechanics, Madeira, Portugal, pp 137143

Malan D, Vogler U, Drescher K (1997) Time-dependent behaviour of hard rock in deep level gold mines. J South African Inst Min Metall 135-147

Malan DF (1999) Time-dependent Behaviour of Deep Level Tabular Excavations in Hard Rock. Rock Mech Rock Eng 32:123-155

Malan DF (1998) An investigation into the identification and modelling of time-dependent behaviour of deep level excavations in hard rock. University of the Witwatersrand

Malan DF, Drescher K (2000) Modeling the post-failure relaxation behaviour of hard rock. In: 4th North American Rock Mechanics Symposium. Seattle, Washington, USA, pp 909-917

Maleki H, Dolinar DR, Dubbert J (2003) Rock Mechanics Study of Lateral Destressing for the Advanceand-Relieve Mining Method. In: 22nd Internatioanl Conference on Ground Control in Mining. Morgantown, WV, USA, pp 105-113

Mark C, Molinda GM, Burke LM (2004) Preventing falls of ground in coal mines with exceptionally lowstrength roof: two case studies. In: 23rd International Conference on Ground Control in Mining. Morgantown, WV

Masuda K (2001) Effects of water on rock strength in a brittle regime. J Struct Geol 23:1653-1657

Mishra B, Verma P (2015) Uniaxial and triaxial single and multistage creep tests on coal-measure shale rocks. Int J Coal Geol 137:55-65

Molinda G, Mark C (2010) Ground failures in coal mines with weak roof. Electron J Geotech Eng 15:547-588

Molinda GM, Oyler DC, Engineer M, Virginia W (2004) Identifying Moisture Sensitive Roof Rocks in Coal Mines. In: 25th International Conference on Ground Control in Mining. pp 57-64

Nadimi S, Shahriar K, Sharifzade M, Moarefvan P (2010) Numerical Analysis of Time-Dependent Behavior of Siah Bisheh Cavern. In: The 6th Asian rock mechanics symposium-advances in rock engineering. pp 23-27

Nadimi S, Shahriar K, Sharifzadeh M, Moarefvand P (2011) Triaxial creep tests and back analysis of time-dependent behavior of Siah Bisheh cavern by 3-Dimensional Distinct Element Method. Tunn 
Undergr Sp Technol 26:155-162

Ndlovu X, Stacey TR (2007) Observations and analyses of roof guttering in a coal mine. J South African Inst Min Metall 107:477-492

Okui Y, Horii H (1997) Stress and time-dependent failure of brittle rocks under compression: A theoretical prediction. J Geophys Res 102:14869

Okui Y, Horii H, Akiyama N (1993) a continuum theory for solids containing microdefects. Internatioanl J Eng Sci 31:735-749

Ottosen NS (1986) Viscoelastic-viscoplastic formulas for analysis of cavities in rock salt. Int J Rock Mech Min Sci Geomech Abstr 23:201-212

Pappas DM, Mark C (2012) Roof and rib fall incident trends: a 10-year profile. Trans Soc Mining, Metall Explor 330:462--478.

Pellet F, Hajdu A, Deleruyelle F, Besnus F (2005) A viscoplastic model including anisotropic damage for the time dependent behaviour of rock. Int J Numer Anal Methods Geomech 29:941-970

Peng S (1973) Time-dependent aspects of rock behavior as measured by a servocontrolled hydraulic testing machine. Int J Rock Mech Min Sci Geomech Abstr 10:235-246

Peng S, Podnieks ER (1972) Relaxation and the behavior of failed rock. Int J Rock Mech Min Sci Geomech Abstr 9:699-712

Perras MA (2009) Tunnelling in horizontally laminated ground; the influence of lamination thickness on anisotropic behaviour and practical observations from the Niagara Tunnel Project. Queen's University

Perzyna P (1966) Fundamental Problems in Nanotechnology. Adv Appl Mech 9:243-377

Price NJ (1964) A study of the time-strain behavior of coal-measure rocks. Int J Rock Mech Min Sci $1: 277-303$

Ramamurthy T, Arora VK (1994) Strength predictions for jointed rocks in confined and unconfined states. Int J Rock Mech Min Sci Geomech Abstr 31:9-22

Ray AK (2009) Influence of cutting sequence and time effects on cutters and roof falls in underground coal mine - numerical approach. WVU

Read HE (1984) Strain softening of rock, soil and concrete - a review artical. Brain Res Rev 3:271-294

Rinne M (2008) Fracture mechanics and subcritical crack growth approach to model time-dependent failure in brittle rock. Helsinki University of Technology

Sano O, Ito I, Terada M (1981) Influence of strain rate on dilatancy and strength of Oshima granite under uniaxial compression. J Geophys Res 86:9299-9311

Schmidtke RH (1985) The long-term strength of Lac du Bonnet Granite. Int J Rock Mech Min Sci Geomech Abstr 22:461-465

Scholz CH (1968) Mechanim of creep in brittle rock. J Geophys Res 73:3295-3302

Schultz RA (1995) Limits on strength and deformation properties of jointed basaltic rock masses. Rock Mech Rock Eng 28:1-15

Schwartz C, Kolluru S (1982) The influence of stress level on the creep of unfilled rock joints. In: The 
23rd U.S Symposium on Rock Mechanics (USRMS). Berkeley, CA, USA, pp 333-340

Schwartz CW, Kolluru S (1981) Laboratory investigation of creep of jointed rock material. In: 22nd US Symposium on Rock Mechanics. Cambridge, Massachusetts, USA, pp 283-289

Shao JF, Chau KT, Feng XT (2006) Modeling of anisotropic damage and creep deformation in brittle rocks. Int J Rock Mech Min Sci 43:582-592

Shao JF, Zhu QZ, Su K (2003) Modeling of creep in rock materials in terms of material degradation. Comput Geotech 30:549-555

Sherizadeh T, Kulatilake PHSW (2016) Assessment of roof stability in a room and pillar coal mine in the U.S. using three-dimensional distinct element method. Tunn Undergr Sp Technol 59:24-37

Shin K, Okubo S, Fukui K, Hashiba K (2005) Variation in strength and creep life of six Japanese rocks. Int J Rock Mech Min Sci 42:251-260

Spene J, Hult J (2001) Simple approximations for creep relaxation. Int J Mech Sci 15:741-755

Stefanizzi S, Barla G, Kaiser PK (2007) Numerical modeling of strain driven fractures around tunnels in layered rock masses. In: 11th Congress of the International Society for Rock Mechanics. Lisbon, Portugal, pp 971-974

Stephansson O (1971) Stability of single openings in horizontally bedded rock. Eng Geol 5:5-71

Sterpi D (1999) An analysis of geotechnical problems involving strain softening effects. Int J Numer Anal Methods Geomech 23:1427-1454

Sterpi D, Gioda G (2009) Visco-Plastic behaviour around advancing tunnels in squeezing rock. Rock Mech Rock Eng 42:319-339

Su WH, Peng SS (1987) Cutter roof and its cause. Min Sci Technol 4:113-132

Sun J, Hu Y. (1997) Time-dependent effects on the tensile strength of saturated granite at Three Gorges Projects in China. Int J Rock Mech Min Sci 34:

US-DOL (2014) MSHA news release: roof fall accidents remain a leading cause of coal mining injuries. https://www.dol.gov/newsroom/releases/msha/msha20141062. Accessed 1 Jan 2016

Van Eckhout E (1976) The mechanisms of strength reduction due to moisture in coal mine shales. Int $\mathbf{J}$ Rock Mech Min Sci Geomech Abstr 13:61-67

Wang Y, Peng SS (1996) High horizontal stress effects on longwall gate entry stability.pdf. In: 15th International Conference on Ground Control in Mining. 13-15 ${ }^{\text {th }}$ Aug. Golden, CO, pp 179-191

Wang Y, Stankus J (1998) Roof control under conditions of shallow depth and high horizontal stress field- a case study. In: 17th International Conference on Ground Control in Mining, West Virginia University, 4-6th Aug. Morgantown, WV, pp 113-118

Wawersik WR (1972) Time-dependent rock behavior in uniaxial compression. In: 14th US Symposium on Rock Mechanics. University Park, PA, USA, pp 85-106

Wawersik WR, Brown WS (1971) Creep fracture in rock in uniaxial compression. Salt Lake City; Utach, USA

Yamaguchi T, Okubo S, Maranini E (2001) Distributions of strength and creep failure time of Inada granite under confing pressure. In: Wang S, Fu B, Li Z (eds) The 2001 ISRM International Symposium - 2nd Asian Rock Mechanics Symposium. Beijing, China, pp 176-180 
Zhang Q, Zhu H, Zhang L, Ding X (2011) Study of scale effect on intact rock strength using particle flow modeling. Int J Rock Mech Min Sci 48:1320-1328 